\title{
Reaktionen des Bis(tert-butyl-methyl)ketazins mit Halogensilanen
}

\author{
Dissertation \\ zur Erlangung des Doktorgrades \\ der Mathematisch-Naturwissenschaftlichen Fakultäten \\ der Georg-August-Universität zu Göttingen
}

vorgelegt von

Nina Armbruster

aus Göttingen

Göttingen 2004 
D 7

Referent:

Prof. Dr. U. Klingebiel

Korreferent:

Prof. Dr. J. Magull

Tag der mündlichen Prüfung:

28.04.2004 

Meiner Familie

Wenn man es nur versucht, so geht's,

das heißt mitunter,

doch nicht stets.

Wilhelm Busch 


\section{Vorwort}

Die vorliegende Arbeit wurde in der Zeit von Juni 2001 bis April 2004 unter der Leitung von Herrn Prof. Dr. U. Klingebiel am Institut für Anorganische Chemie der Georg-August-Universität Göttingen angefertigt.

Herrn Prof. Dr. U. Klingebiel danke ich besonders für das interessante und herausfordernde Thema dieser Arbeit, die Bereitstellung eines Arbeitsplatzes und die allzeit gewährte Unterstützung und Betreuung.

Für NMR-Messungen und die speziellen Diskussionen und Anregungen in Bezug auf meine Arbeit danke ich Herrn Dr. G. Elter.

Meiner Arbeitsgruppe danke ich für das angenehme Arbeitsklima. Besonderer Dank geht dabei an meine Freunde Suse, Bernd, Thomas und Verena.

Dem technischen Personal möchte ich für ihre Hilfsbereitschaft und stete Zusammenarbeit danken.

Der größte Dank gilt aber meiner Familie, insbesondere Florian, die mir immer zur Seite stand.

Vielen Dank! 


\section{Abkürzungsverzeichnis}

Äq. Äquivalent, Äquivalente

ABX, AA'XX' Spektrum höherer Ordnung

br. breites NMR-Signal

$\mathrm{Bu}$

Butyl $\left(-\mathrm{C}_{4} \mathrm{H}_{9}\right)$

$\mathrm{BuH}$

Butan

BuLi

Butyllithium

${ }^{\circ} \mathrm{C}$

Grad Celsius

d

Dublett

DSCP

Diazasilacyclopenten bzw. Dihydrodiazasilol

dvd

Dublett von Dublett

EI

Elektronenstoßionisation

Et

Ethyl $\left(-\mathrm{C}_{2} \mathrm{H}_{5}\right)$

$\mathrm{eV}$

Elektronenvolt

$\mathrm{FI}$

Feldionisation

gem.

Gemäß

$\mathrm{h}$

hour, Stunde

Hal

Halogen

$\mathrm{HV}$

Hausvakuum ( 30 mbar)

$\mathrm{Hz}$

Hertz

iPr

Isopropyl $\left(-\mathrm{CH}\left(\mathrm{CH}_{3}\right)_{2}\right)$

$\mathrm{J}$

Kopplungskonstante

$[\mathrm{M}]^{+}$

Molekülion

$\mathrm{m}$

Multiplett

mbar

Millibar

$\mathrm{Me}$

Methyl $\left(-\mathrm{CH}_{3}\right)$

$\mathrm{mL}$

Milliliter

$n$

normal, unverzweigt

ND

Normaldruck (1013 mbar)

NMR

Nuclear magnetic resonance, Kernspinresonanzspektroskopie

ÖV

Ölpumpenvakuum ( 10-2 mbar)

$\mathrm{Ph}$

Phenyl $\left(-\mathrm{C}_{6} \mathrm{H}_{5}\right)$

$\mathrm{pm}$

Picometer

ppm

parts per million 


$\begin{array}{ll}\text { pst } & \text { pseudo-Triplett } \\ \mathrm{q} & \text { Quartett } \\ \mathrm{R} & \text { beliebiger organischer Rest } \\ \mathrm{RT} & \text { Raumtemperatur }\left(\sim 20^{\circ} \mathrm{C}\right) \\ \mathrm{s} & \text { Singulett } \\ \text { sbr } & \text { sehr breites NMR-Signal } \\ \text { Schmp. } & \text { Schmelzpunkt } \\ \text { Sdp. } & \text { Siedepunkt } \\ \text { sept } & \text { Septett } \\ \text { Subp. } & \text { Sublimationspunkt } \\ \mathrm{t} & \text { Triplett } \\ t \text {-Bu } & \text { tertiär-Butyl }\left(-\mathrm{C}\left(\mathrm{CH}_{3}\right)_{3}\right) \\ \text { TMP } & 2,2,6,6-\text { Tetramethyl-piperidin } \\ t e r t & \text { tertiär } \\ \text { THF } & \text { Tetrahydrofuran }\left(\mathrm{C}_{4} \mathrm{H}_{8} \mathrm{O}\right) \\ \text { TMS } & \text { Tetramethylsilan }\left(\mathrm{Si}\left(\mathrm{CH}_{3}\right)_{4}\right) \\ \text { vgl. } & \text { vergleiche, verglichen mit } \\ \mathrm{X}, \mathrm{Y} & \text { beliebiges Element } \\ \Sigma & \text { Summe } \\ \Sigma & \text { Winkelsumme } \\ \circ & \text { Grad } \\ \ddagger & \text { nicht isoliertes } \mathrm{Zwischenprodukt} \\ \delta & \text { chemische Verschiebung } \\ \Delta & \text { Wärmezufuhr }\end{array}$

Die vorgestellten Verbindungen werden fortlaufend nummeriert und fettgedruckt. Bekannte Verbindungen werden römisch, neu isolierte Verbindungen arabisch beziffert.

Die Verbindungen sind nach den Regeln der IUPAC in Bezug auf Alkane und Silane benannt, z. B. 1,2-Diaza-3-sila-5-cyclopenten bzw. 3,4-Dihydro-1,2,3-diazasilol. Im Text werden beide Namen parallel verwendet. 


\section{Inhaltsverzeichnis}

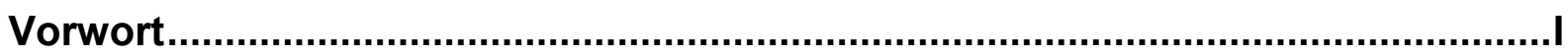

Abkürzungsverzeichnis ..............................................................................

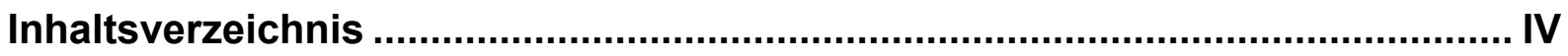

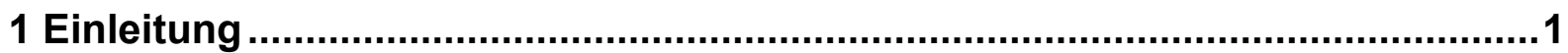

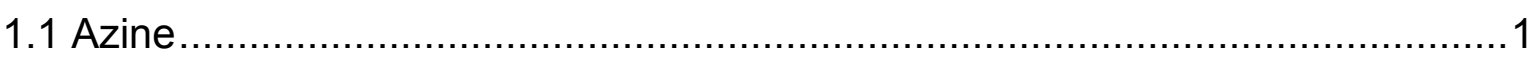

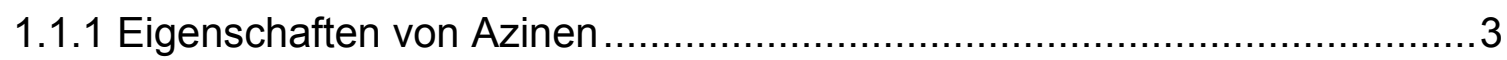

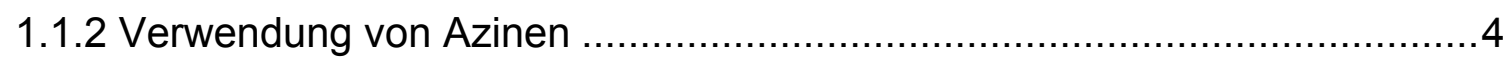

1.1 .3 Isomerie der Azine ....................................................................... 4

1.1.4 Ausgewählte Reaktionen............................................................. 5

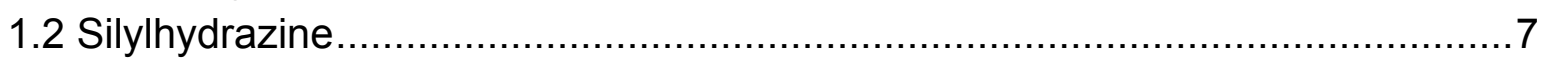

1.2.1 Synthese und Eigenschaften von Silylhydrazinen ................................ 7

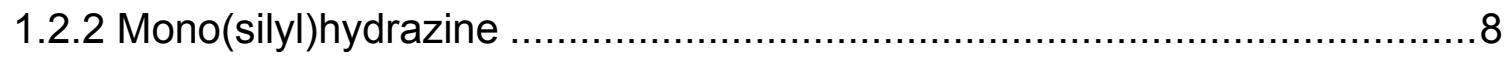

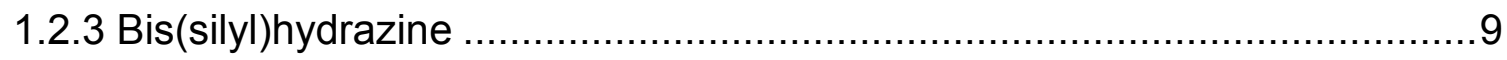

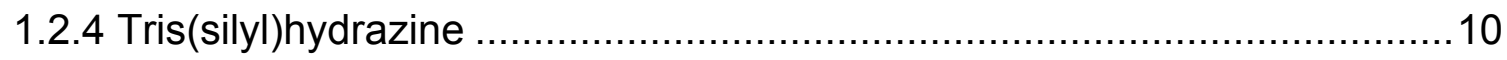

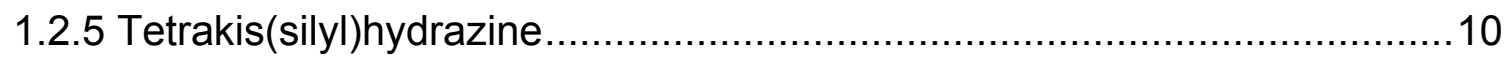

1.2.6 Ringe und Hydrazinosilane ..................................................... 10

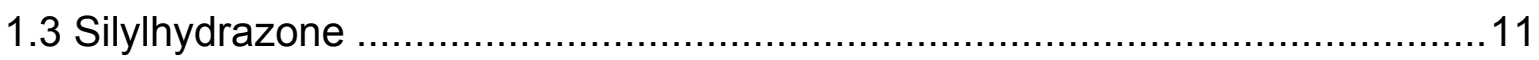

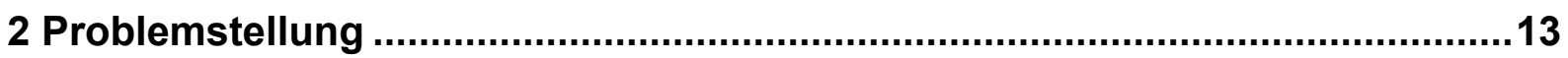

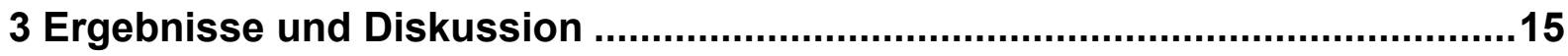

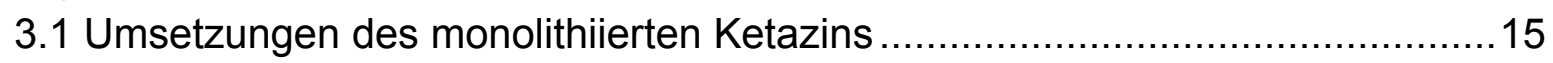

3.1.1 Reaktionen mit Chlorsilanen .................................................... 15

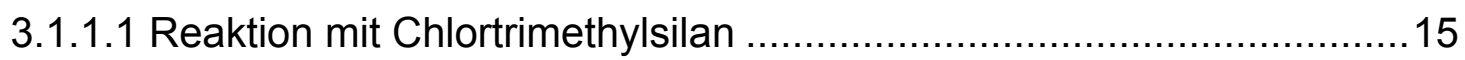

3.1.1.2 Chlorsilylketazine und Bis(ketazinyl)silane ................................. 16

3.1.1.3 Ein Lithium-Hydrazonid-THF-Addukt ........................................ 19

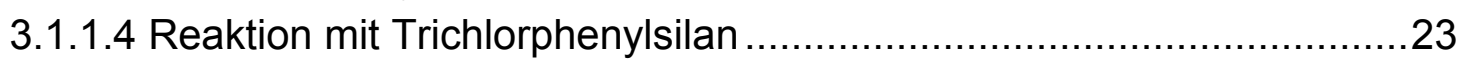

3.1.1.5 Umsetzung mit Tetrachlorsilan .............................................. 23

3.1.2 Reaktionen mit Fluorsilanen ......................................................... 25

3.1.2.1 Umsetzungen mit Monofluorsilanen........................................ 25

3.1.2.2 Synthese von isomeren cyclischen Silylketazinen ..........................25

3.1.2.3 Ein acyclisches Fluorsilylketazin .............................................. 39

3.1.2.4 Reaktion mit Tetrafluorsilan ................................................... 42

3.2 Umsetzungen des dilithiierten Ketazins ............................................44

3.2.1 Reaktionen mit Chlorsilanen ...................................................... 44

3.2.1.1 Reaktionen mit Dichlorsilanen ................................................... 44

3.2.1.2 Dichlorfunktionelle cyclische Ketazine .....................................46

3.2.2 Reaktionen mit Fluorsilanen ....................................................... 48

3.2.2.1 Umsetzungen mit Difluorsilanen ............................................... 48

3.2.2.2 Synthese von fluorfunktionellen Diazasilacyclopentenen ..................54

3.2.2.3 Reaktionen mit Tetrafluorsilanen ..............................................59

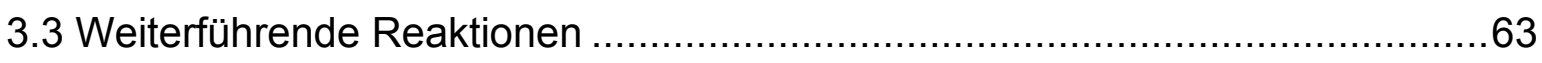

3.3.1 Reaktion von Verbindung 12 mit Methanol....................................63 
3.3.2 Versuche zur Bildung einer Si=C-Doppelbindung .............................64

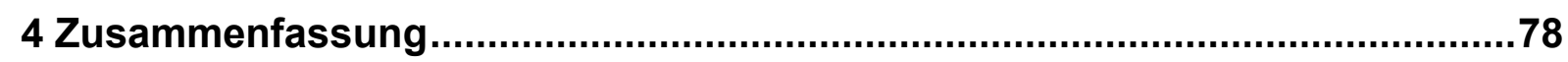

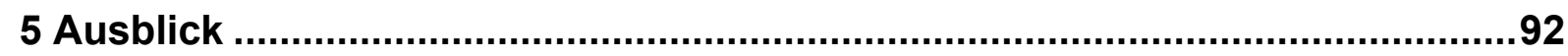

6 Präparativer Teil ......................................................................................94

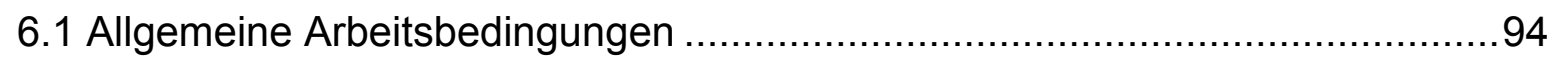

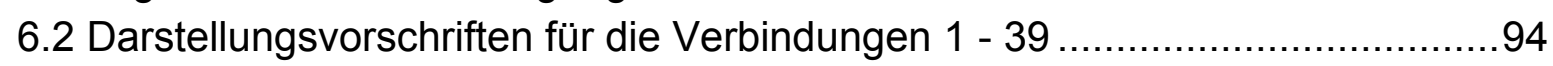

6.3 Behandlung und Entsorgung der Abfälle ............................................. 101

7 Charakteristische Daten der dargestellten Verbindungen.............................102

7.1 Namen, Siede- und Schmelzpunkte, Ausbeuten sowie massen- und

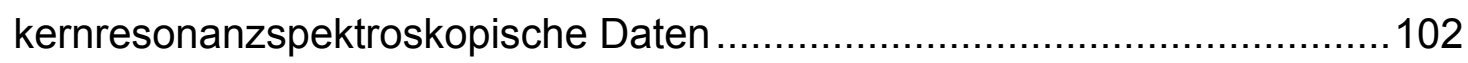

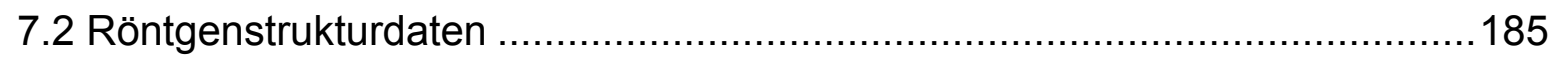

7.3 Verzeichnis der neuen Verbindungen ............................................... 194

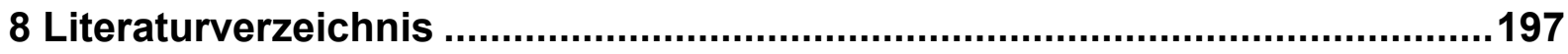

Publikationen ..............................................................................................201

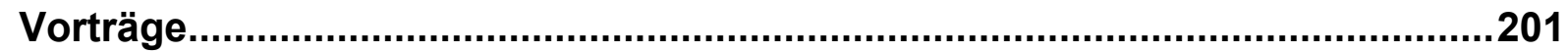

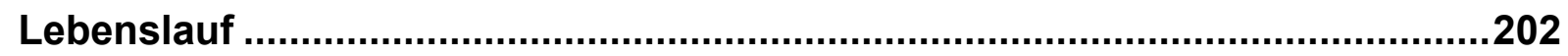





\section{Einleitung}

\subsection{Azine}

Als Azine bezeichnet man Hydrazinderivate mit der allgemeinen Struktur ${ }^{[1]}$ :<smiles>[R]C([R])=NN=C([R])[R]</smiles>

Abbildung 1: Allgemeine Struktur eines Azins

Die Reste $R_{1}, R_{2}, R_{3}$ und $R_{4}$ stehen für Wasserstoff, Alkyl-, Aryl- oder Acylgruppen. Azine sind Derivate des Hydrazins. Sie werden üblicherweise durch Kondensationsreaktionen von Hydrazin und Carbonylverbindungen hergestellt.

Prinzipiell werden Azine in Aldazine und Ketazine unterteilt, je nach dem ob man ein Aldehyd oder Keton als Edukt verwendet. Nach Anordnung der Substituenten unterscheidet man weiterhin zwischen symmetrischen und unsymmetrischen Azinen.
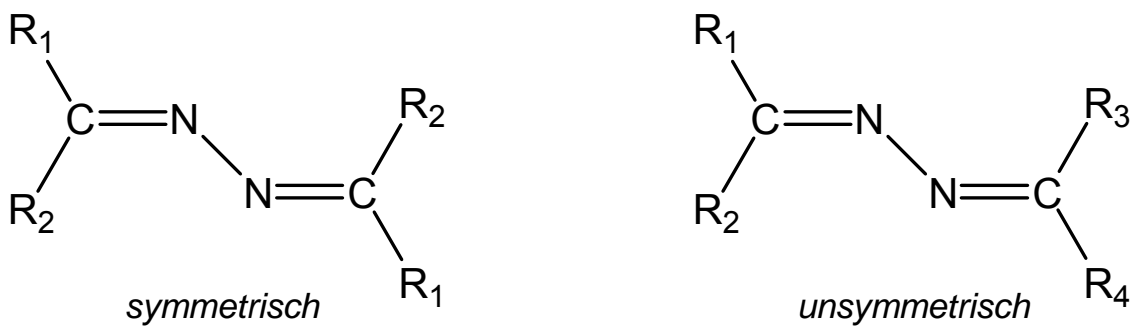

Abbildung 2: Symmetrische und unsymmetrische Azine

Da sich diese Arbeit ausschließlich mit symmetrischen Ketazinen beschäftigt, wird auf weitere Ausführungen über asymmetrische Ketazine und die Aldazine verzichtet. 
Symmetrische Ketazine lassen sich durch Kondensation von Ketonen mit Hydrazinhydrat, -sulfat oder -hydrochlorid in wässriger oder organischer, z. B. alkoholischer Lösung darstellen. Zum Teil wird auch mit wasserfreiem Hydrazin ohne Lösungsmittel gearbeitet (siehe Schema 1).

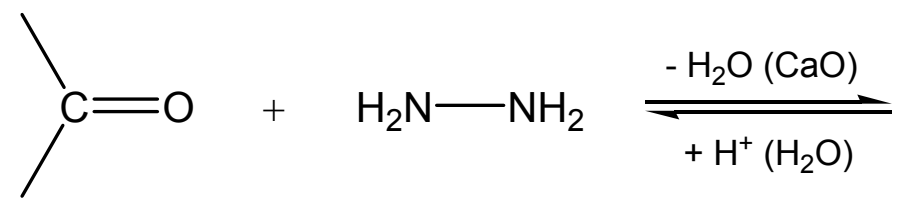

Hydrazin

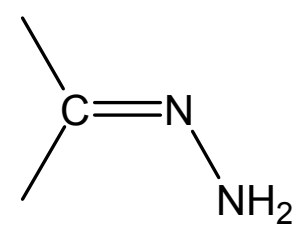

Hydrazon

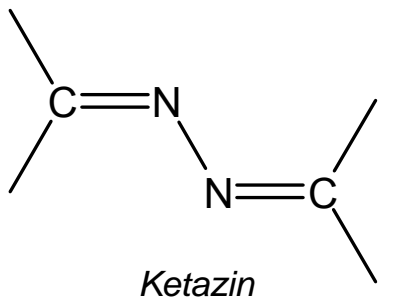

Ketazin

Die Darstellung von Azinen ausgehend von Dialkylketonen erfolgt im allgemeinen ohne Schwierigkeiten. Entstehen bei der Umsetzung zunächst die entsprechenden Hydrazone ${ }^{[2]}$, kann man diese durch Zugabe einiger Tropfen Säure (Essigsäure oder Salzsäure) in das Reaktionsgemisch und eventuell erhöhter Temperatur in die Ketazine überführen.

Weniger leicht erfolgt die Synthese der Azine von Alkylarylketonen und besonders von Diarylketonen, bei denen schärfere Reaktionsbedingungen gewählt werden müssen. Es kann folgende Reihe für die Reaktionsfähigkeit von Carbonylverbindungen mit Hydrazin aufgestellt werden:

Aldehyde $>$ Dialkylketone $>$ Alkylarylketone $>$ Diarylketone 
Weitere Methoden für die Herstellung von Azinen ${ }^{[3]}$ sind z. B. die Umsetzung von Methylenverbindungen mit Distickstoffoxid und die Reaktion von Carbonylverbindungen mit Ammoniak in Gegenwart eines Oxidationsmittels und eines Katalysators. Die zuletzt genannte Methode wird zur Synthese von symmetrischen Azinen verwendet. Als Oxidationsmittel nutzt man häufig $70 \%$ iges Wasserstoffperoxid und als Katalysator das Dinatriumsalz der Ethylendiamintetraessigsäure und Kieselgel. Die gebräuchlichsten Lösungsmittel sind Acetonitril und Isopropanol.

\subsubsection{Eigenschaften von Azinen}

Die Stoffklasse der Azine wurde von T. CURTIUS 1889 entdeckt und untersucht ${ }^{[3]}$. Azine aliphatischer Aldehyde und Ketone sind farblose oder schwach gefärbte Flüssigkeiten mit charakteristischem Geruch. Die Siedepunkte steigen mit zunehmender Molmasse der Substituenten $\mathrm{R}_{1}-\mathrm{R}_{4}$. Aromatische oder heterocyclische Azine sind dagegen Feststoffe. Azine lösen sich meistens in einer Reihe von organischen Lösungsmitteln, aber nicht in Wasser.

Nahezu alle Azine zeigen charakteristische Absorptionen im UV-Spektrum. Sie sind in der Lage intramolekulare Wasserstoffbrücken zu bilden, was mit der Fluoreszenzfähigkeit und der Komplanarität der Atome in der Azin-Gruppe im Zusammenhang steht ${ }^{[1]}$.

Zahlreiche Azine wurden auf ihre antibakterielle Wirkung und tuberkulostatische Aktivität getestet. Diese konnten für ein paar von ihnen nachgewiesen werden. Außerdem fand man heraus, dass einige Azine enzymatische Reaktionen stimulieren und andere für die Herstellung anticancerogener Substanzen eingesetzt werden können. Bestimmte Ketazine hemmen die Aktivität der Monoaminooxydase und damit den Abbau von Noradrenalin, Dopamin und Serotonin. Sie wurden als eine neue Klasse von MAO-Hemmern zu pharmakologischen Prüfungen empfohlen. 


\subsubsection{Verwendung von Azinen}

Azine kommen in unterschiedlichen Industriezweigen zum Einsatz ${ }^{[1]}$. Aromatische und aliphatische Azine werden als Stabilisatoren in Benzin, Seifen, chlorierten Kohlenwasserstoffen, Silberhalogenid-Emulsionen und Industrieölen verwendet. Ferner finden Azine Anwendung bei der Herstellung von Harzen und Lacken, da sie mit Metallen stabile Komplexe bilden. Bei der Verarbeitung von Kunststofffasern, -geweben und -folien erhöhen sie deren Festigkeit und Dehnbarkeit.

In der analytischen Chemie werden Azine als Reagenzien zum Nachweis verschiedener Elemente oder Verbindungsklassen eingesetzt. Darüber hinaus eignen sie sich als Indikatoren bei potentiometrischen Titrationen.

\subsubsection{Isomerie der Azine}

Die Isomerie der Azine entspricht der $\mathrm{C}=\mathrm{N}-$ Isomerie $^{[4]}$. Sowohl die Imin-EnaminTautomerie als auch die photochemische (E/Z)-Isomerisierung sind untersucht worden. Änderungen der chemischen Eigenschaften beruhen auf der syn- und antiKonfiguration der Azine:

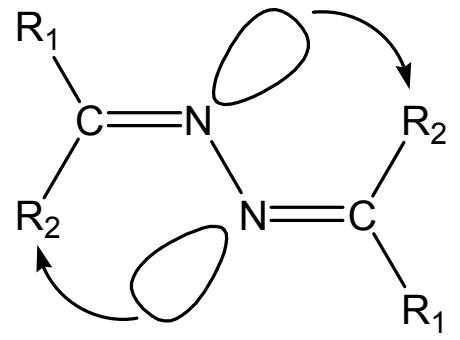

Anti-Anti-

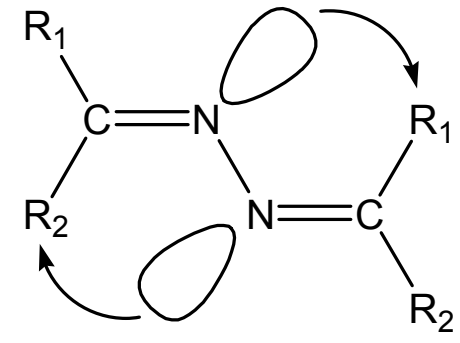

Anti-Syn-

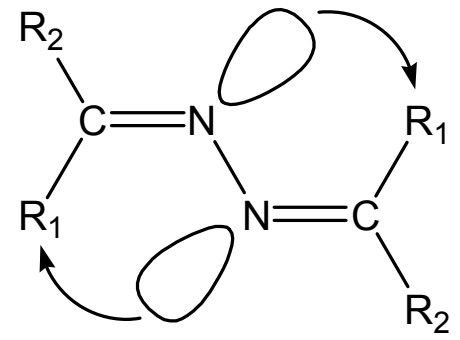

Syn-Syn-

Abbildung 3: Isomere bei symmetrischen Azinen

Die Isomere werden bezüglich der Nähe des freien Elektronenpaares am Stickstoff zu $\mathrm{R}_{1}$ als Anti-Anti-, Anti-Syn- und Syn-Syn-Konformere bezeichnet ${ }^{[5,6]}$.

Sterisch anspruchsvolle Substituenten können die Isomerenbildung allerdings verhindern. Setzt man beispielsweise für $R_{1}$ eine Ethyl- und $R_{2}$ eine Methylgruppe als Substituenten ein, treten alle Isomere auf ${ }^{[5]}$. Erhöht man den sterischen Anspruch 
und verwendet statt einer Ethylgruppe für $\mathrm{R}_{1}$ eine Isopropylgruppierung, erhält man die Isomere Anti-Anti : Anti-Syn im Verhältnis $9: 1^{[5]}$.

Als Ausgangsverbindung für diese Arbeit wurde das Bis(tert-butyl-methyl)ketazin gewählt. Der sterische Anspruch der tert-Butylgruppe unterbindet die Bildung eines Isomerengemisches. Es entsteht nur das Anti-Anti-Isomer ${ }^{[7]}$.

\subsubsection{Ausgewählte Reaktionen}

Bei Anwesenheit von Mineralsäuren bilden Azine Azinium-Salze:<smiles>[R]C([R])=NN=C([R])[R]</smiles><smiles>[X]C1CC1[NH2+]C</smiles><smiles>[Y]CC([R2])=N[NH2+]C([R])[R]</smiles>
$\mathrm{X}=\mathrm{Cl}, \mathrm{Br}, \mathrm{HSO}_{4}$

Gleichung 1: Bildung von Azinium-Salzen

In der Azinchemie spielen auch Cycloadditionen, besonders 1,3-dipolare Cycloadditionen, eine bedeutende Rolle.

Ketazine mit einem aciden $\mathrm{H}$-Atom am a-C-Atom, wie z. B. das Bis(tert-butylmethyl)ketazin, bilden unter Einwirkung starker Basen 1,6-Dianionen, die zu neuen Azin-Derivaten umgesetzt werden können.

Acetophenonazin wurde 1969 von $\mathrm{HENOCH}^{[8]}$ mit Benzylchlorid alkyliert:<smiles>C/C(=N/N=C(\C)c1ccccc1)c1ccccc1</smiles>

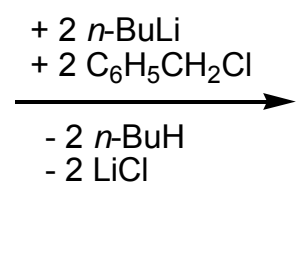<smiles>c1ccc(CC/C(=N/N=C(\CCc2ccccc2)c2ccccc2)c2ccccc2)cc1</smiles> 
Lithiumsalze des Ketazins konnten bisher nicht isoliert oder kristallisiert werden. Allerdings sind Folgeprodukte bekannt, die ihre Existenz bestätigen. Umsetzungen der Dianionen mit Halogenboranen wurden im Arbeitskreis MELLER von $\mathrm{GROH}^{[9,10]}$ und ARMBRUSTER ${ }^{[11]}$ untersucht:
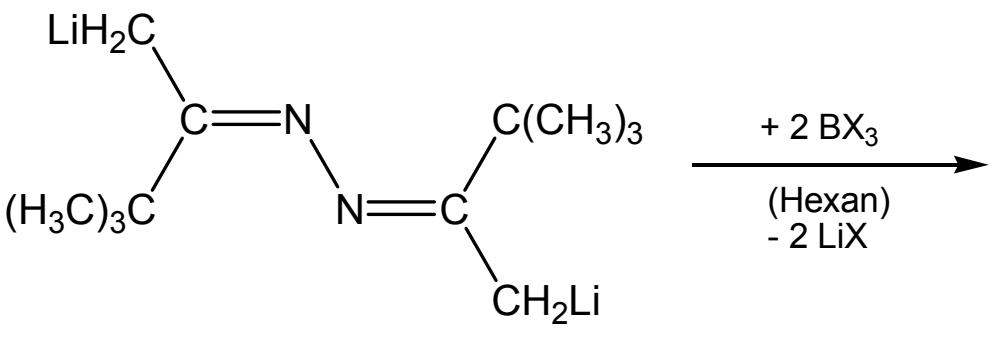

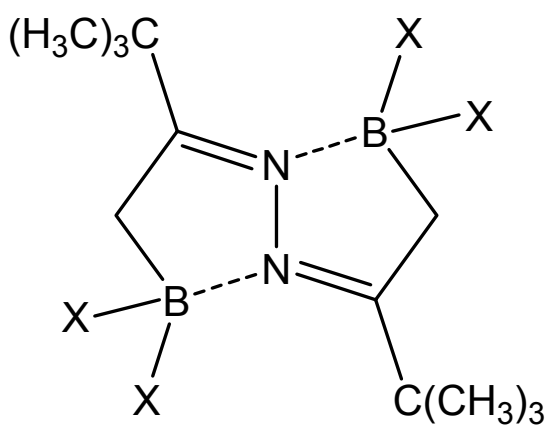

$\mathrm{X}=\mathrm{F}, \mathrm{Cl}, \mathrm{Br}, \mathrm{I}$

Gleichung 3: Eine Reaktion des Bis(tert-butyl-methyl)ketazins mit Halogenboranen

Die einzige bisher durchgeführte Reaktion des Bis(tert-butyl-methyl)ketazins mit Halogensilanen ist die Umsetzung mit Chlortrimethylsilan von $\mathrm{GROH}^{[10] \text { : }}$

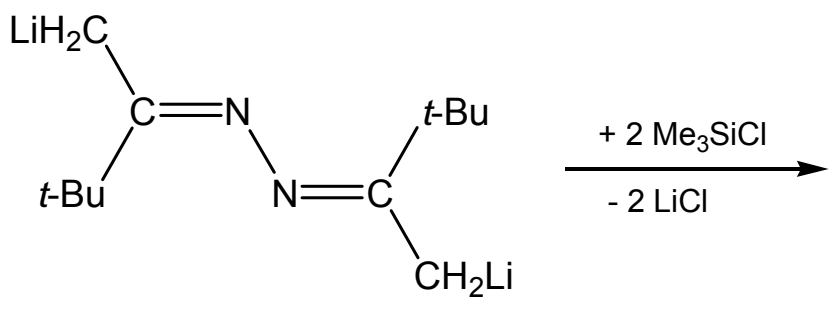

Gleichung 4: Darstellung des 1,4-Di-tert-butyl-1,4-bis(trimethylsilyl)ketazins II

Die Ketazine sind hinsichtlich der Siliciumchemie ein neues Arbeitsgebiet. Dagegen sind Reaktionen des aus der Ketazindarstellung bekannten Hydrazins und der Hydrazone mit Halogensilanen bereits gut untersucht. 


\subsection{Silylhydrazine}

\subsubsection{Synthese und Eigenschaften von Silylhydrazinen}

Die ersten cyclischen und acyclischen Silylhydrazine wurden von AYLETT ${ }^{[12]}$ und WANNAGAT et al. ${ }^{[13,14]}$ 1956-1958 beschrieben. Sie entwickelten im wesentlichen zwei Darstellungsmethoden:

A) Reaktion von wasserfreiem Hydrazin mit Halogensilanen $(\mathrm{Hal}=\mathrm{Cl}, \mathrm{Br}, \mathrm{I})$

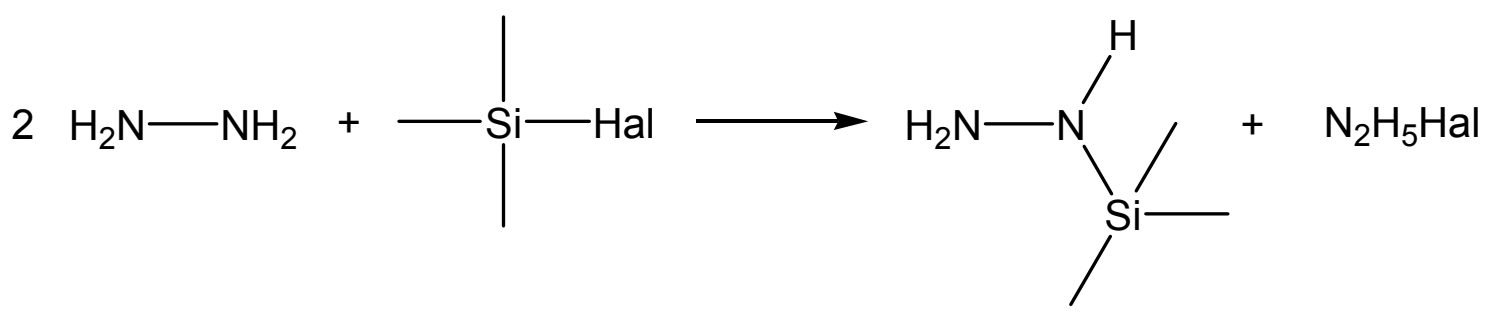

Schema 2: Reaktionsweg A

Reaktionsweg A (Schema 2) ist die gebräuchlichste Methode für die Reaktion mit Chlor-, Brom- oder lodsilanen ${ }^{[15]}$. Häufig werden noch Triethylamin oder Pyridine als Base hinzugefügt.

B) Fluorsilane bilden aufgrund ihrer reduzierten Reaktivität mit Hydrazinen Addukte. Es muss deshalb ein anderer Syntheseweg gewählt werden:<smiles>C[SiH2]C(C)(C)[SiH2]N</smiles>

Schema 3: Reaktionsweg B

Lithiumhydrazide werden mit Halogensilanen, speziell mit Fluorsilanen, unter Lithiumhalogenid-Abspaltung nach Schema 3 umgesetzt. 


\subsubsection{Mono(silyl)hydrazine}

Die Bildung von Tris-, Bis- bzw. Monosilylhydrazinen ist abhängig von der Reaktivität und der Größe der Silylsubstituenten. Der Oligomerisationsgrad nimmt dabei mit steigender Größe der Silylsubstituenten ab ${ }^{[12,13,14,16]}$.

Setzt man Hydrazin mit lodsilan oder Brommethylsilan um, erhält man das Tetrakis(silyl)hydrazin ${ }^{[12,16]}$. Die Reaktion mit Chlordimethylsilan ergibt nur das Tris(silyl)hydrazin ${ }^{[16]}$. Bei der Umsetzung von Trimethylsilanen erhält man ein Isomerengemisch aus $N, N$ - und $N, N^{\prime}$-Bis(trimethylsilyl)hydrazinen ${ }^{[14]}$.

Das bis 1993 einzige Mono(silyl)hydrazin war das Triphenylsilylhydrazin. Es wurde 1958 von WANNAGAT und LIEHR vorgestellt ${ }^{[13]}$.

$2 \mathrm{H}_{2} \mathrm{~N}-\mathrm{NH}_{2}+\mathrm{Ph}_{3} \mathrm{SiCl}$

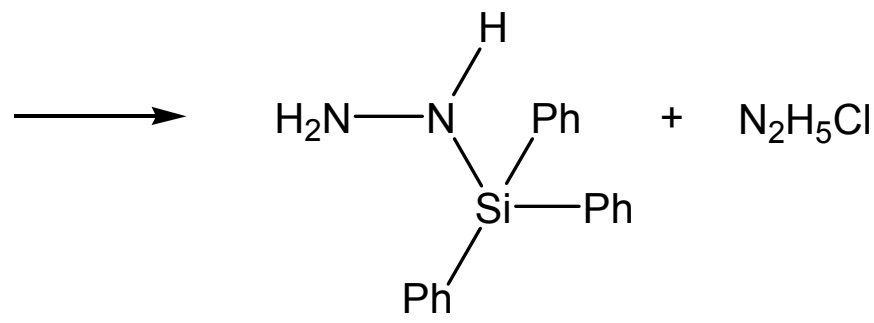

Gleichung 5: Darstellung des ersten Mono(silyl)hydrazins

Weitere stabile Mono(silyl)hydrazine konnten später von KLINGEBIEL et al. ${ }^{[17,18,19,20,21,22]}$ durch die Reaktion von Fluorsilanen mit lithiierten Hydrazinen synthetisiert werden:

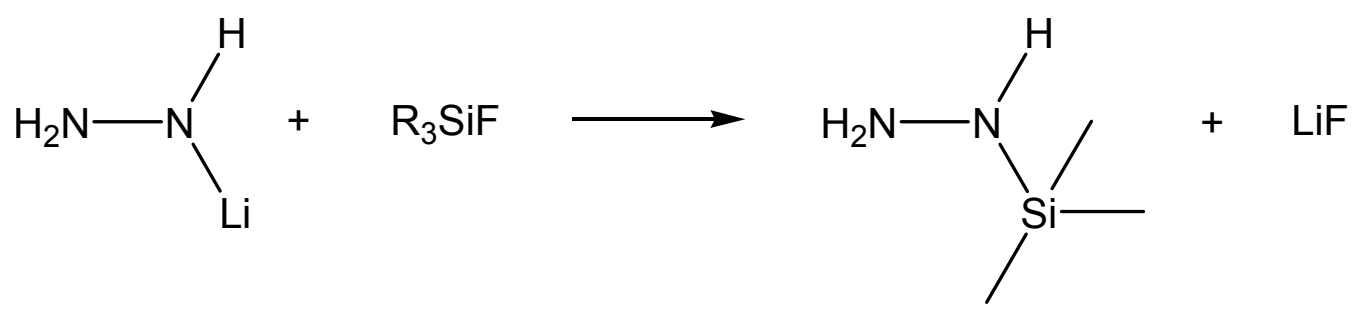

Schema 4: Synthese weiterer stabiler Mono(silyl)hydrazine

Sie werden kinetisch durch sterisch anspruchsvolle tert-Butyl- oder Amingruppen stabilisiert. 


\subsubsection{Bis(silyl)hydrazine}

Instabile Mono(silyl)hydrazine kondensieren schon bei Raumtemperatur zu Bis(silyl)hydrazinen:

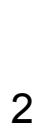<smiles></smiles><smiles>O=[N+][O-]</smiles><smiles>[R3][SiH2]N([2H])N([R5])[SiH3]</smiles>

Gleichung 6: Bildung von symmetrischen Bis(silyl)hydrazinen

Diese Kondensationsreaktionen führen häufig zur Bildung von

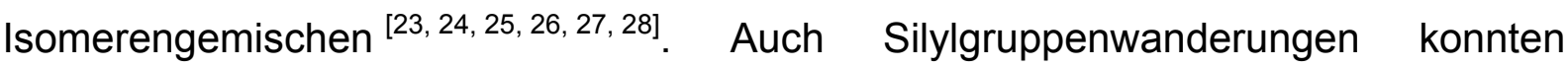
erstmals bei größeren Silylsubstituenten nachgewiesen werden [24, 25, 26, 29, 30, 31, 32, 33].

Asymmetrische Bis(silyl)hydrazine entstehen entweder durch Umsetzung von Hydrazinen mit einem Gemisch verschiedener Halogensilane (Reaktionsweg A), oder durch die Reaktion der Lithiumderivate von stabilen Mono(silyl)hydrazinen mit einem Halogensilan (Reaktionsweg B):

$$
\mathrm{H}_{2} \mathrm{~N} \longrightarrow \mathrm{NH}_{2}+\mathrm{R}_{3} \mathrm{SiHal}+\mathrm{R}_{3}{ }_{3} \mathrm{SiHal} \underset{-2 \mathrm{HHal}}{\longrightarrow}
$$<smiles>[R3][SiH2]NN([R3])[AsH]</smiles>

Reaktionsweg A<smiles>[Y5]N([Hg])[TlH]</smiles><smiles>[R3][SiH2-]</smiles><smiles>[I-][I-]</smiles><smiles>[R15]NN([R5])[SiH2]</smiles>

Reaktionsweg B

Gleichung 7: Darstellung von asymmetrischen Bis(silyl)hydrazinen 


\subsubsection{Tris(silyl)hydrazine}

Die Synthese von Tris(silyl)hydrazinen gelingt auf zwei verschiedenen Wegen: Zum einen über die Lithiierung von Bis(silyl)hydrazinen und Reaktion mit Halogensilanen. Dabei können Produkte mit drei unterschiedlichen Silylgruppen isoliert werden ${ }^{[14]}$; oder Hydrazin wird in Anwesenheit von Triethylamin mit Chlorsilanen umgesetzt ${ }^{[16]}$ :

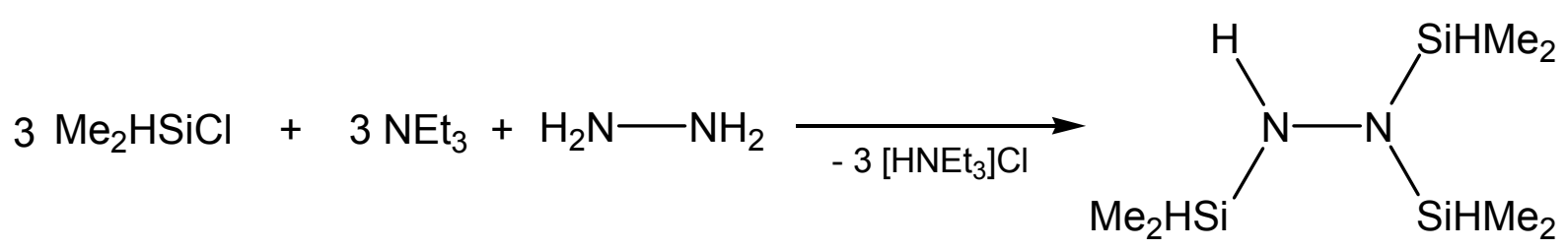
Gleichung 8: Darstellung von Tris(silyl)hydrazinen

\subsubsection{Tetrakis(silyl)hydrazine}

Tetrakis(silyl)hydrazine werden über vier verschiedene Syntheserouten erhalten:
A) Eine Reaktion von lodsilan und Hydrazin in der Gasphase ${ }^{[12]}$,
B) eine Zersetzung von Diiminen ${ }^{[34,35]}$,
C) eine Dehydrohalogenierung ${ }^{[16,36]}$ (vgl. Tris(silyl)hydrazine Gleichung 8) und
D) die bekannte Methode über das Lithiumderivat (vgl. Gleichung 7) ${ }^{[20,37,38,39]}$.

\subsubsection{Ringe und Hydrazinosilane}

Für die Darstellung von cyclischen Silylhydrazinen hat sich die intra- oder intermolekulare LiF-Abspaltung aus den Lithium-fluorsilylhydraziden als eine schonende und in guten Ausbeuten verlaufende Methode erwiesen. Isoliert wurden drei-, vier-, fünf- und sechsgliedrige Ringe ${ }^{[19,40]}$. Auch Bis- und Tris(hydrazino)silane konnten bereits hergestellt werden $\left.{ }^{[17,} 20,37,41\right]$. 


\subsection{Silylhydrazone}

Die klassische Synthese der N-Organylhydrazone basiert auf der Wasserkondensation aus Carbonylen und Hydrazinen (siehe Schema 1). NSilylhydrazone sind auf diesem Weg nicht zugänglich. Sie wurden Ende der siebziger Jahre im Arbeitskreis KLINGEBIEL auf anderem Wege dargestellt ${ }^{[42,43,44,45,46] \text { : }}$<smiles>[R]C([NH3+])=NN([R])Cl</smiles>

Organylhydrazid<smiles>[R2][Si]([R])(F)F</smiles><smiles>F[I-]</smiles>

Gleichung 9: Darstellung von Silylhydrazonen über Organylhydrazone

Silylhydrazone lassen sich außerdem über die entsprechenden Silylhydrazine herstellen. Sie werden mit einem Keton zur Reaktion gebracht und durch $\mathrm{H}_{2} \mathrm{O}-$ Kondensation entsteht das Silylhydrazon ${ }^{[47]}$ :<smiles>CN(N)[Si](C)(C)C</smiles>

Silylhydrazin

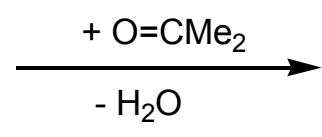

Gleichung 10: Synthese von Silylhydrazonen ausgehend von Hydrazinen

Die Fluorfunktionalität von Fluorsilylhydrazonen kann zum Aufbau von Bis-, Tris-, Tetrakis(hydrazonyl)silanen, Bis(imino)cyclodisilazanen und N-silylsubstituierten 1,2Diaza-3-sila-5-cyclopentenen (DSCP) bzw. 3,4-Dihydro-1,2,3-diazasilolen genutzt werden. 
Fluorsilylhydrazone haben bei Anwesenheit acider Allylprotonen weitere lithiierbare Zentren. Diese führen durch intramolekulare Lithiumfluorid-Abspaltungen zu Cyclisierungen, wodurch 1,2-Diaza-3-sila-5-cyclopentene entstehen:<smiles>[R]/C(C[AlH2])=N/N([R])[Si]([R1])([R])F</smiles>

Fluorsilylhydrazon<smiles>[R]C1=NN([R])[Si]([R])([R])C1</smiles>

DSCP

Gleichung 11: Cyclisierung von Fluorsilylhydrazonen zu Diazasilacyclopentenen

DSCP stellen eine interessante Verbindungsklasse dar, die bereits eingehend in Substitutions- ${ }^{[48,49]}$, Eliminierungs- ${ }^{[50,51]}$ und Radikalreaktionen ${ }^{[48]}$ untersucht wurde. Es gelang auch die Darstellung von Tricyclen ${ }^{[50]}$, Spiroverbindungen ${ }^{[48,51,52]}$ sowie die Synthese von Phosphaethenen ${ }^{[53]}$ mit exocyclischer Doppelbindung.

Da die Ketazine Derivate des Hydrazins darstellen, liegt der Gedanke nahe, zu überprüfen, ob sich die bekannte Chemie der Silylhydrazine und -hydrazone auf Ketazine übertragen lässt. 


\section{Problemstellung}

Das Bis(tert-butyl-methyl)ketazin besitzt am a-C-Atom ein acides $\mathrm{H}$-Atom und bildet unter Einwirkung starker Basen 1,6-Dianionen. In der Literatur sind bisher nur sehr wenige Reaktionen von Halogensilanen mit Ketazinen beschrieben ${ }^{[10,11]}$. Daher sollte die Synthese einer Vielzahl unbekannter Silylketazine möglich sein.

Untersucht werden soll die Auswirkung des sterischen Anspruchs der Halogensilane auf die entstehenden Produkte. Des weiteren stellt sich die Frage, in wie weit man durch Wahl der Reaktionsbedingungen die Produktbildung steuern kann.

Hydrazone können in 1- und 4-Position metalliert werden und cyclisieren zu Diazasilacyclopentenen. Ketazine dagegen bilden 1,6-Dianionen und sollten deshalb siebengliedrige Ringe bilden:
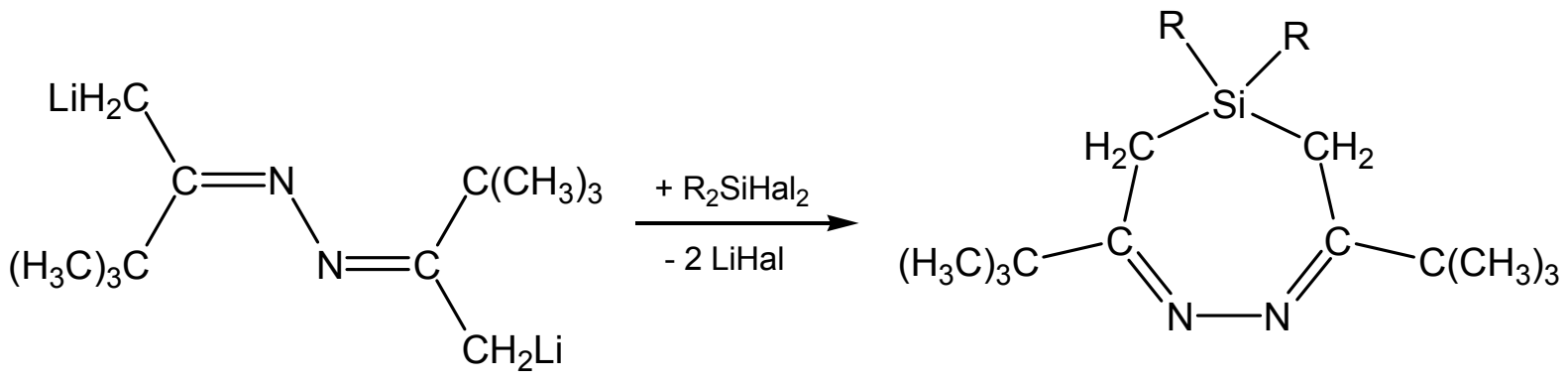

Gleichung 12: Denkbarer Reaktionsverlauf eines dilithiierten Ketazins mit Dihalogensilanen

Die Umsetzung des dilithiierten Bis(tert-butyl-methyl)ketazins mit halogenfunktionellen Amino-, Aryl- und Aryloxyboranen führt allerdings zur Bildung von Diazaboracyclopentenen ${ }^{[10]}$ (siehe Gleichung 13).
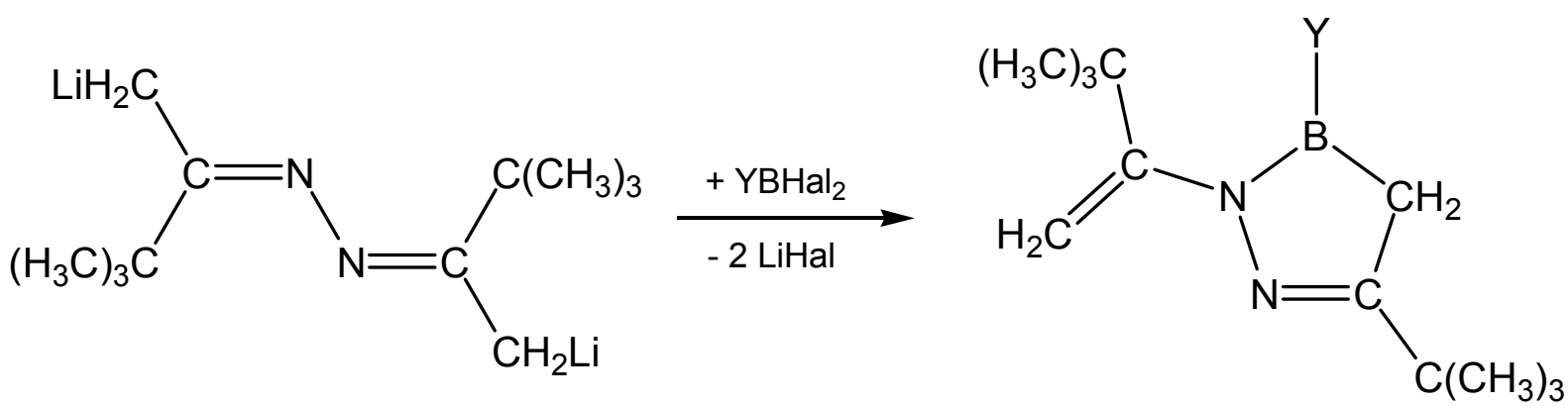

Gleichung 13: Reaktion des Bis(tert-butyl-methyl)ketazins mit Halogenboranen 
Aus diesem Grund liegt ein weiterer Schwerpunkt der Arbeit darin zu klären, ob und in welcher Weise mit Silylketazinen Heterocyclen aufgebaut werden können.

Besitzen die entstehenden Ringsysteme noch acide Wasserstoffatome, ist auch die Folgechemie von Interesse. Dies ist von cyclischen Silylhydrazonen bekannt ${ }^{[48,50,52]}$. Bei entsprechender Dehydrohalogenierung könnten die Silylketazine als Precursoren für Si=C-Doppelbindungen in Frage kommen. In Phospholen bzw. 1,2,3-Diazaphosphacyclopentadienen sind $\mathrm{P}=\mathrm{C}$-Doppelbindungen bekannt, die durch das aromatische 6rT-System stabilisiert werden und z. B. gegenüber Luftsauerstoff, Feuchtigkeit und Schwefel unempfindlich sind ${ }^{[54]}$. 


\section{Ergebnisse und Diskussion}

Das Bis(tert-butyl-methyl)ketazin I vereint zwei wesentliche Eigenschaften: Die Methylgruppen sind metallierbar und die tert-Butylgruppen besitzen einen stabilisierenden Effekt für die Folgeprodukte. Außerdem wird durch diese Substituentenwahl die Ausbildung eines Isomerengemisches verhindert, was die Interpretation der NMR-Daten vereinfacht. Aus diesen Gründen wurde das Bis(tertbutyl-methyl)ketazin für diese Arbeit als Ausgangsverbindung gewählt. Die Darstellung erfolgt in Anlehnung an HOUBEN-WEYL ${ }^{[1]}$ durch die Umsetzung von tertButyl-methylketon mit Hydrazin-Monohydrat ohne Lösungsmittel:

$\left(\mathrm{H}_{3} \mathrm{C}\right)_{3} \mathrm{C}$

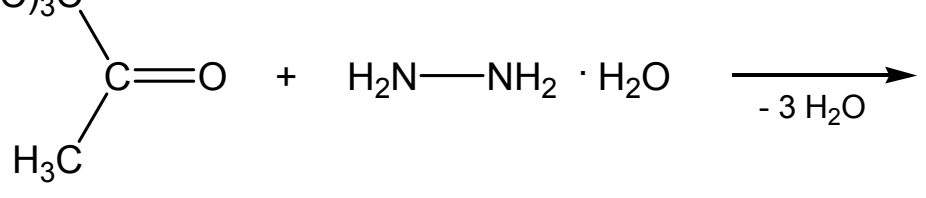

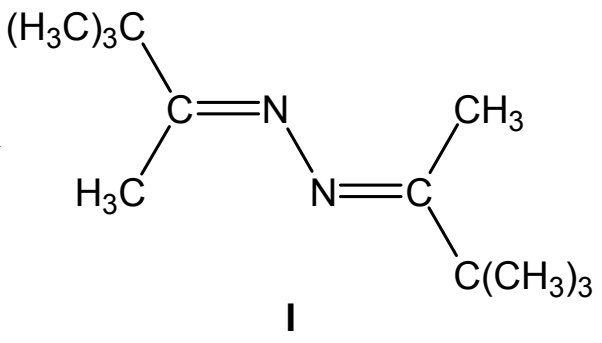

Gleichung 14: Darstellung des Bis(tert-butyl-methyl)ketazins

\subsection{Umsetzungen des monolithiierten Ketazins}

\subsubsection{Reaktionen mit Chlorsilanen}

\subsubsection{Reaktion mit Chlortrimethylsilan}

Wird das Bis(tert-butyl-methyl)ketazin bei $0{ }^{\circ} \mathrm{C}$ in $\alpha$-Stellung im Verhältnis $1: 1 \mathrm{mit}$ lithiumorganischen Reagenzien metalliert und anschließend mit Chlortrimethylsilan umgesetzt, entsteht in guter Ausbeute das 2-(3,3-Dimethyl-1-(trimethylsilyl)butan-2yliden)-1-(3,3-dimethylbutan-2-yliden)hydrazin 1: 

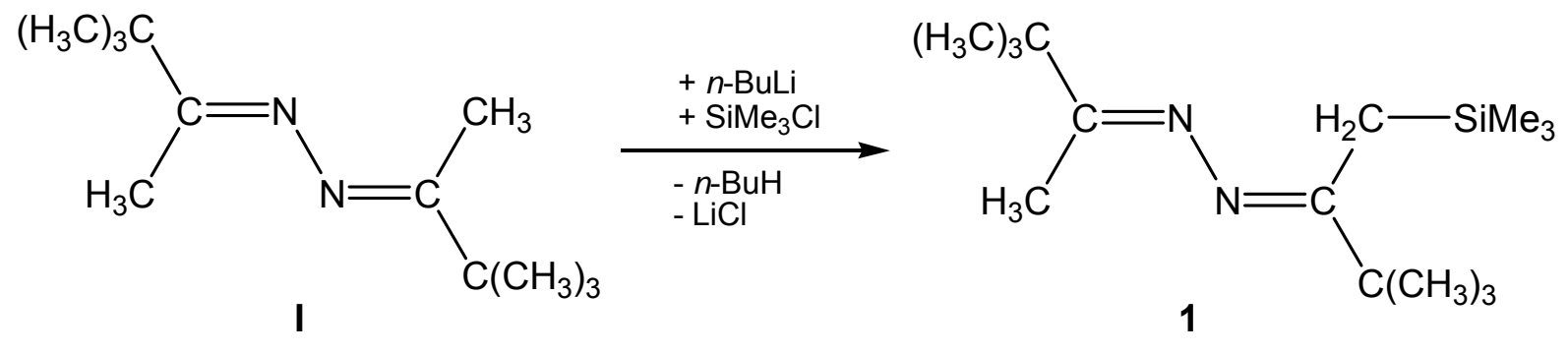

Gleichung 15: Reaktion des monolithiierten Ketazins mit Chlortrimethylsilan

Ein monosilyliertes Ketazin konnte auch im Arbeitskreis MELLER ${ }^{[11]}$ durch die Reaktion von Bis(isopropyl-methyl)ketazin mit Chlortrimethylsilan dargestellt werden. Beide monosilylierten Ketazine sind hellgelbe Flüssigkeiten, die sich im Vakuum unzersetzt destillieren lassen.

\subsubsection{Chlorsilylketazine und Bis(ketazinyl)silane}

Die Reaktion von monolithiiertem Bis(tert-butyl-methyl)ketazin mit Dichlorsilanen im molaren Verhältnis 1 : 1 führt zu den chlorfunktionellen Ketazinen 2 und $\mathbf{3}$ :

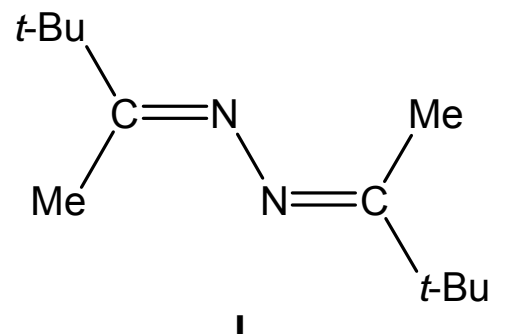

I

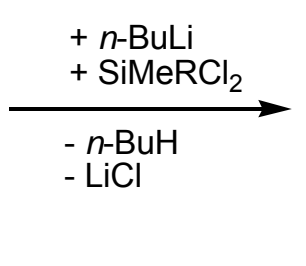

\begin{tabular}{|c|c|c|}
\hline Verb. & $\mathbf{2}$ & $\mathbf{3}$ \\
\hline $\mathbf{R}$ & $\mathrm{Me}$ & $\mathrm{Ph}$ \\
\hline
\end{tabular}<smiles>[R][Si]([R])(Cl)C/C(=N/N=C(/C)C(C)(C)C)C(C)(C)C</smiles>

2, 3

Gleichung 16: Umsetzungen des monolithiierten Ketazins mit Dichlorsilanen

Während die Reaktion von I mit Dichlormethylphenylsilan zu 3 in relativ guter Ausbeute verläuft, erhält man Verbindung 2 nur zu ca. $10 \%$. Als Nebenreaktion tritt hier eine intramolekulare Cyclisierung auf. Die entstehende Verbindung konnte gezielt dargestellt werden und wird in Kapitel 3.2.1.1 genauer behandelt. Eine solche Cyclisierung tritt bei Verbindung 3 nur nach erneuter Umsetzung mit tert-Butyllithium auf (siehe ebenfalls Kapitel 3.2.1.1). 
Die Verbindungen 2 und 3 sind hydrolyseempfindliche gelbe Flüssigkeiten. Der + Mund - I-Effekt des Phenylrestes von Verbindung 3 bewirkt im Vergleich zum Alkylrest der Verbindung 2 eine Verschiebung des ${ }^{29}$ Si-NMR-Signals von 28,7 ppm (Verbindung 2) auf 16,4 ppm (Verbindung 3).

Der Versuch einer Dehydrohalogenierung von Verbindung 3 durch Zugabe der Base Triethylamin schlug fehl.

Setzt man das Monolithiumsalz des Bis(tert-butyl-methyl)ketazins bzw. der Verbindung 1 mit Dichlordimethylsilan im Verhältnis 2:1 um, entstehen die Bis(ketazinyl)silane Bis(2-(2-(3,3-dimethylbutan-2-yliden)hydrazono)-3,3-dimethylbut1-yl)-dimethylsilan 4 und Bis(2-(2-(3,3-dimethyl-1-(trimethylsilyl)butan-2-yliden)hydrazono)-3,3-dimethylbut-1-yl)-dimethylsilan 5 :

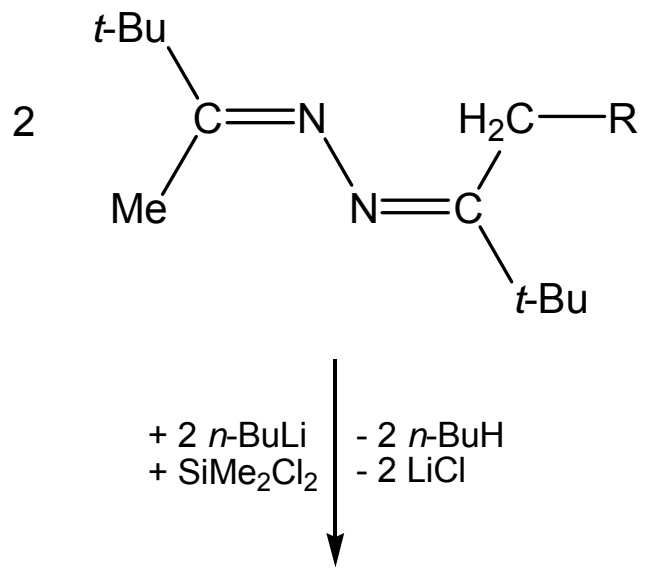<smiles>[R]CC(C[Si](C)(C)CC(=NN=C(C[R])CCCC)C(C)(C)C)=NN=C(C[R])C(C)(C)C</smiles>

\section{4,5}

\begin{tabular}{|c|c|c|}
\hline Verb. & $\mathbf{4}$ & $\mathbf{5}$ \\
\hline $\mathbf{R}$ & $\mathrm{H}$ & $\mathrm{SiMe}_{3}$ \\
\hline
\end{tabular}


Die Verbindungen $\mathbf{4}$ und $\mathbf{5}$ sind gelbe, sehr zähe Flüssigkeiten, die jeweils in einer Ausbeute von rund $30 \%$ erhalten werden.

Analoge Hydrazonverbindungen wurden im Arbeitskreis KLINGEBIEL ${ }^{[47]}$ beispielsweise durch die Umsetzung von Di-tert-butyldifluorsilan mit Diphenylhydrazon dargestellt:<smiles>CC(C)(C)[Si](F)(F)C(C)(C)C</smiles><smiles>CC(C)(C)[Si](NN=C(c1ccccc1)c1ccccc1)(NN=C(c1ccccc1)c1ccccc1)C(C)(C)C</smiles>

Gleichung 18: Darstellung eines Bis(hydrazonyl)silans

Bis(hydrazino)silane sind schon seit 1960 bekannt ${ }^{[55,56,57]}$. Sie wurden in der Reaktion von Diorganodichlorsilanen mit organischen Hydrazinderivaten erhalten. Sie zeigen eine hohe Hydrolyseempfindlichkeit und neigen zu Kondensationsreaktionen. Bis(hydrazino)silane, die am terminalen Stickstoff silyl-substituiert ${ }^{[20,21,40,58]}$ sind, haben eine höhere Stabilität. Eine Methode, diese zu synthetisieren ist die Umsetzung eines Silylhydrazins mit Dichlordimethylsilan: 


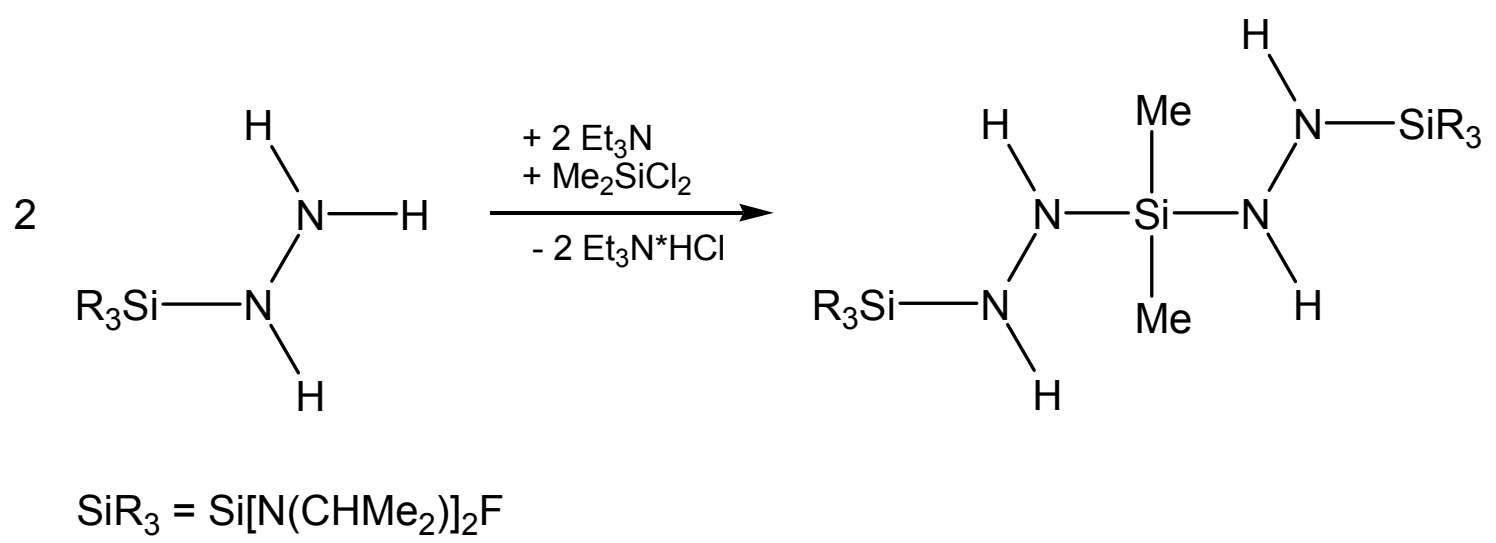

Gleichung 19: Darstellungsmethode für Bis(hydrazino)silane

\subsubsection{Ein Lithium-Hydrazonid-THF-Addukt}

Bei der Reaktion der metallierten Verbindung I mit Di-tert-butyldichlorsilan entsteht in einer Ausbeute von ca. $10 \%$ Verbindung 6:<smiles>CC(C)(C)C(C)(C)C</smiles>

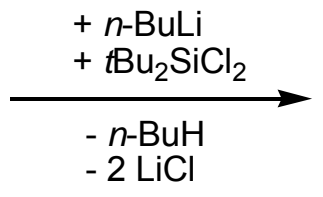

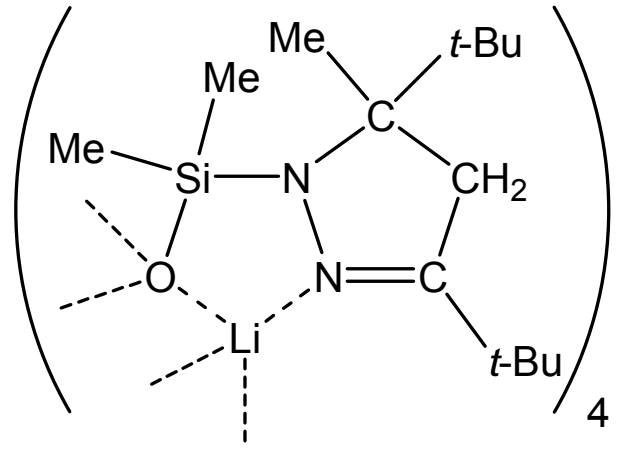

6

Gleichung 20: Darstellung eines Lithium-Hydrazonid-THF-Adduktes

Der Reaktionsverlauf und die Genese des Produktes sind weitgehend unklar. Durch Umkristallisation in einem $n$-Hexan / THF-Gemisch wurden röntgentaugliche Kristalle erhalten. 


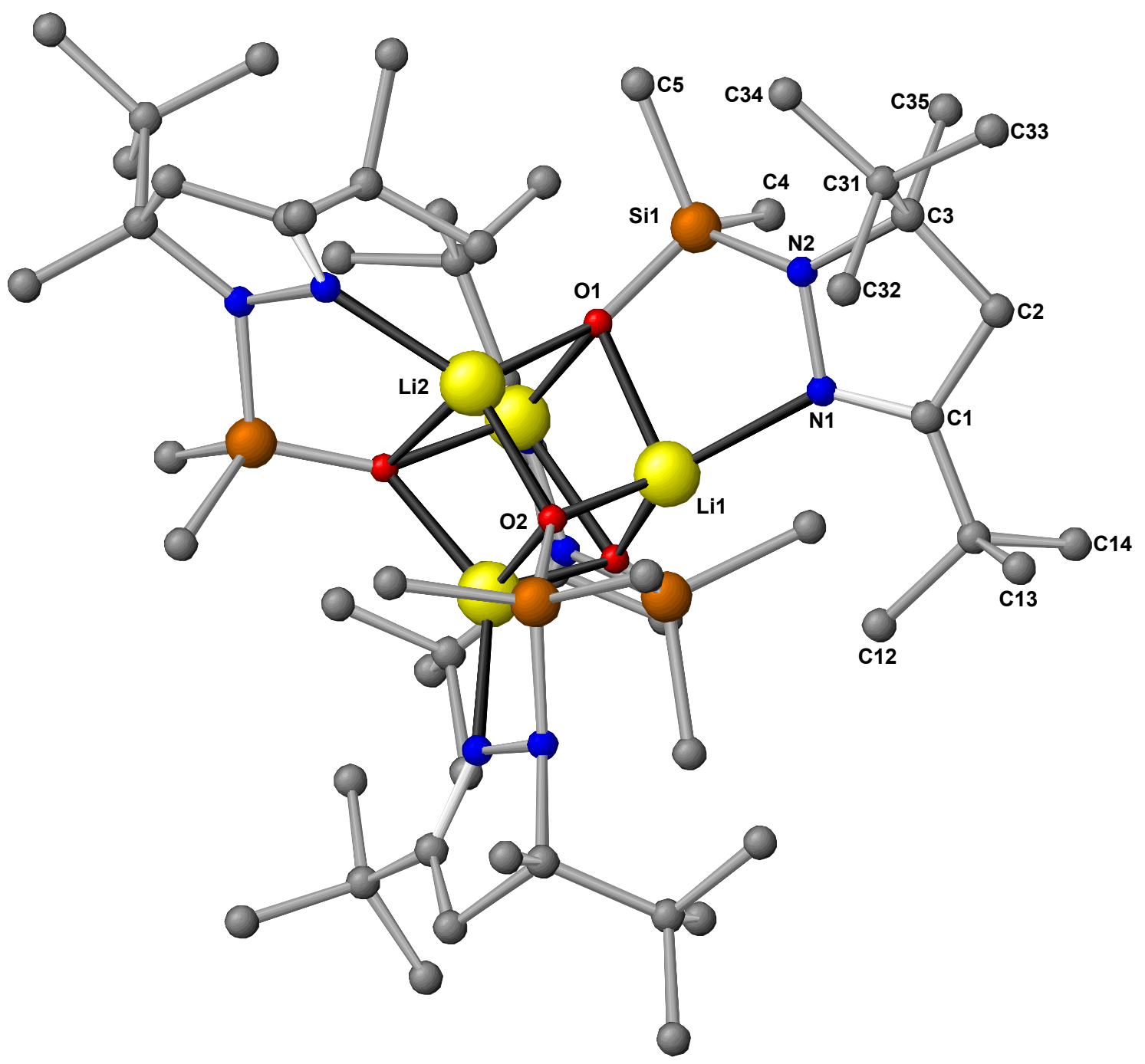

Abbildung 4: Kristallstruktur von Verbindung 6

Verbindung 6 kristallisiert in der monoklinen Raumgruppe C2/c. Die Abbildungen 4-7 geben die Struktur von 6 im Festkörper wieder. Die Tabellen 1-3 enthalten ausgewählte Bindungslängen und -winkel. Eine Elementarzelle von 6 beinhaltet 4 Moleküle. Die zentrale Einheit bildet ein Li-O-Kuban, welches über vier Li-O-Kanten je ein Ringsystem fixiert. So ist jedes Lithium- und jedes Sauerstoffion vierfach koordiniert. Das Ringsystem besteht aus einem 1,2-Azaazoniacyclopenten-2 (N2-N1C1-C2-C3), welches über das N2-Dimethylsilylen mit einem Sauerstoffatom des Kubans verbrückt ist. Weiterhin wird der fünfgliedrige Ring über eine N1-Li1Adduktbindung an der zentralen Einheit fixiert. Die Bindungslängen von 6 liegen alle im zu erwartenden Bereich. Die N1-C1-Bindung beträgt $128 \mathrm{pm}$ und ist somit eine typische $\mathrm{C}=\mathrm{N}$-Doppelbindung. Der N2-N1-Abstand liegt mit einer Länge von 140 pm im Bereich einer N-N-Einfachbindung. 
Abbildung 5 zeigt einen Ausschnitt des beschrifteten Teils aus Abbildung 4 mit seitlichem Blick auf die Ringebene des Azaazoniacyclopentens. Die Alkylsubstituenten wurden der Übersichtlichkeit halber weggelassen. Der Ausschnitt beinhaltet drei miteinander verknüpfte Ringe, die im Folgenden einzeln betrachtet werden. Das 1,2-Azaazoniacyclopenten-2 (N2-N1-C1-C2-C3) bildet einen nahezu planaren Ring, aus dessen Ebene das C3-Atom um 21 pm herausragt. Die Summe der Innenwinkel weicht mit $538,2^{\circ}$ um $1,8^{\circ}$ von der Planarität ab.

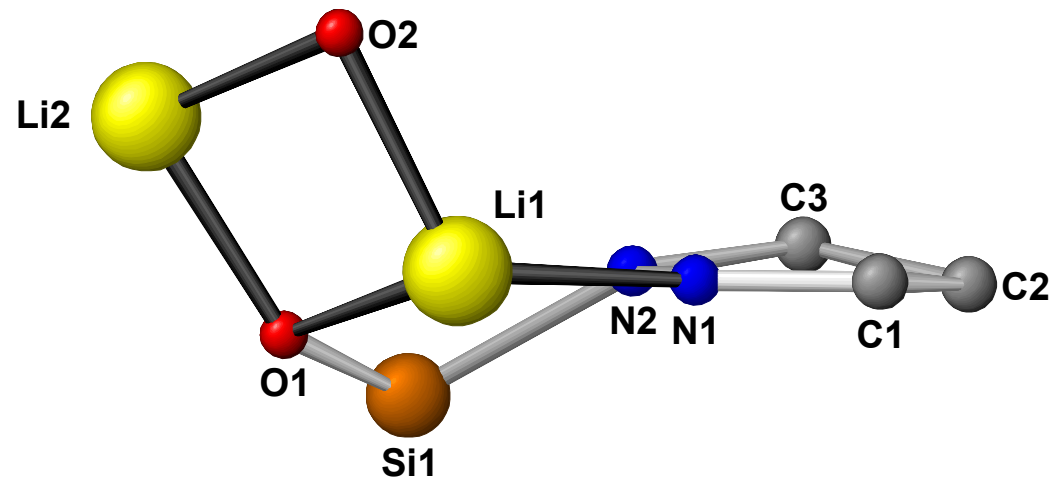

Abbildung 5: Ebene des N2-N1-C1-C2-C3-Ringes von Verbindung 6

\begin{tabular}{|l|r|}
\hline N2-N1 & $140,0 \mathrm{pm}$ \\
\hline N1-C1 & $128,9 \mathrm{pm}$ \\
\hline C1-C2 & $150,5 \mathrm{pm}$ \\
\hline C2-C3 & $151,3 \mathrm{pm}$ \\
\hline C3-N2 & $151,3 \mathrm{pm}$ \\
\hline
\end{tabular}

\begin{tabular}{|l|l|}
\hline N2-N1-C1 & $111,3^{\circ}$ \\
\hline N1-C1-C2 & $111,3^{\circ}$ \\
\hline C1-C2-C3 & $104,4^{\circ}$ \\
\hline C2-C3-N2 & $101,8^{\circ}$ \\
\hline C3-N2-N1 & $109,4^{\circ}$ \\
\hline$\Sigma^{\circ}$ & $538,2^{\circ}$ \\
\hline
\end{tabular}

Tabelle 1: Ausgewählte Bindungslängen und - winkel des N2-N1-C1-C2-C3-Ringes von Verbindung 6

Aus der von N2-N1-Li1 aufgespannten Ebene ragt Si1 um 96,6 pm und O1 um 56,2 pm heraus. Die Winkelsumme an N1 bestätigt mit 359,9 ${ }^{\circ}$ die $\mathrm{sp}^{2}$-Hybridisierung und die trigonal-planare Geometrie. Mit 352,5 ${ }^{\circ}$ weicht die Winkelsumme von N2 davon deutlich ab. Si1 ist tetraedrisch umgeben, was die Winkelsumme von 656,9 ${ }^{\circ}$ verdeutlicht. Der zweite Ring ähnelt somit in seiner Konformation der einer „Halbsessel-Konformation“ des Cyclopentans. 


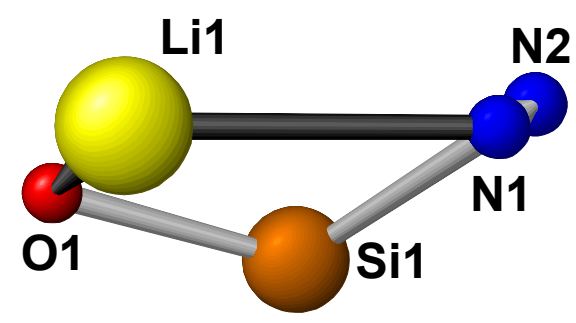

Abbildung 6: Ebene des N2-N1-Li1-O1-Si1-Ringes von Verbindung 6

\begin{tabular}{|l|r|}
\hline N2-N1 & $140,0 \mathrm{pm}$ \\
\hline N1-Li1 & $210,5 \mathrm{pm}$ \\
\hline Li1-O1 & $199,1 \mathrm{pm}$ \\
\hline O1-Si1 & $157,8 \mathrm{pm}$ \\
\hline Si1-N2 & $179,6 \mathrm{pm}$ \\
\hline
\end{tabular}

\begin{tabular}{|l|r|}
\hline N2-N1-Li1 & $109,6^{\circ}$ \\
\hline N1-Li1-O1 & $90,3^{\circ}$ \\
\hline Li1-O1-Si1 & $109,1^{\circ}$ \\
\hline O1-Si1-N2 & $106,6^{\circ}$ \\
\hline Si1-N2-N1 & $109,0^{\circ}$ \\
\hline$\Sigma^{\circ}$ & $524,6^{\circ}$ \\
\hline
\end{tabular}

Tabelle 2: Ausgewählte Bindungslängen und - winkel des N2-N1-Li1-O1-Si1-Ringes von Verbindung 6

Der dritte Ring beschreibt eine Ebene des Kubans und weist eine leicht gefaltete Konformation auf. Die Abweichung der Innenwinkelsumme von der eines Quadrates beträgt $1,3^{\circ}$. Die Fläche des Kubans ist um $45^{\circ}$ an der Li1-O1-Kante gegenüber der Ebene des benachbarten Rings geknickt (vgl. auch Abbildung 5).

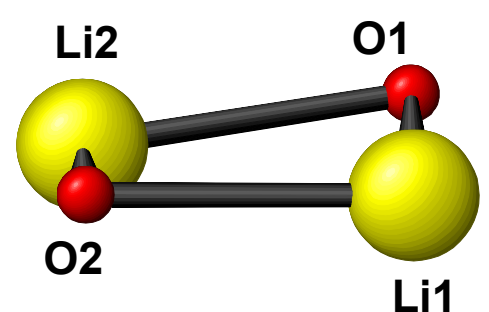

Abbildung 7: Ebene des O1-Li1-O2-Li2-Ringes von Verbindung 6

\begin{tabular}{|l|r|}
\hline O1-Li1 & $199,1 \mathrm{pm}$ \\
\hline Li1-O2 & $192,7 \mathrm{pm}$ \\
\hline O2-Li2 & $195,5 \mathrm{pm}$ \\
\hline Li2-O1 & $194,9 \mathrm{pm}$ \\
\hline
\end{tabular}

\begin{tabular}{|l|r|}
\hline O1-Li1-O2 & $98,0^{\circ}$ \\
\hline Li1-O2-Li2 & $81,8^{\circ}$ \\
\hline O2-Li2-O1 & $98,5^{\circ}$ \\
\hline Li2-O1-Li1 & $80,4^{\circ}$ \\
\hline$\Sigma^{\circ}$ & $358,7^{\circ}$ \\
\hline
\end{tabular}

Tabelle 3: Ausgewählte Bindungslängen und - winkel des O1-Li1-O2-Li2-Ringes von Verbindung 6 


\subsubsection{Reaktion mit Trichlorphenylsilan}

Wird das monolithiierte Bis(tert-butyl-methyl)ketazin mit einem Äquivalent Trichlorphenylsilan versetzt, entsteht in relativ guter Ausbeute das monosilylierte acyclische Ketazin 7:<smiles>CC(C)(C)C(C)(C)C</smiles>

I

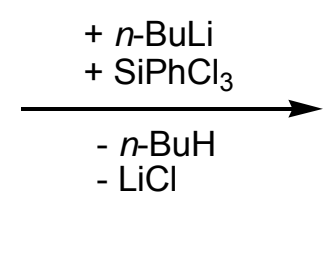<smiles>CC(C)(C)C(C)(C)C</smiles>

7

Gleichung 21: Reaktion eines monolithiierten Ketazins mit Trichlorphenylsilan

Verbindung 7 ist eine hellgelbe Flüssigkeit, die eine hohe Empfindlichkeit gegenüber Hydrolyse aufweist.

Der Versuch, Verbindung 7 mit tert-Butyllithium umzusetzen (vgl. Verbindung 3), führte zu keinem eindeutigen Ergebnis. Es entsteht ein Produktgemisch mit hohem polymeren Anteil. Vermutlich konkurriert hier die Substitutionsreaktion am Silicium mit der Metallierung der a-ständigen Kohlenstoffe des Ketazins.

\subsubsection{Umsetzung mit Tetrachlorsilan}

Das einfach lithiierte Bis(tert-butyl-methyl)ketazin reagiert mit Tetrachlorsilan im molaren Verhältnis 1:1 zum 2-(1-(Trichlorsilyl)-3,3-dimethylbutan-2-yliden)-1-(3,3dimethylbutan-2-yliden)hydrazin 8:<smiles>CC(C)(C)C(C)(C)C</smiles>

I<smiles>CC(=NN=C(C[Si](Cl)(Cl)Cl)C[As](C)(C)C)C(C)(C)C</smiles>

8

Gleichung 22: Reaktion des Monolithiumsalzes mit Tetrachlorsilan 
Bei Verbindung 8 handelt es sich, wie bei den meisten Ketazinen, um eine hellgelbe Flüssigkeit, die aufgrund der drei Chlorfunktionen extrem hydrolyseempfindlich ist.

Bei der thermischen Aufarbeitung aller Chlorsilylketazine kann es zu einer Abspaltung von Chlorwasserstoff kommen. Durch intra- und intermolekulare Kondensationsreaktionen und die Bildung von Aziniumsalzen ${ }^{[1]}$ entstehen zahlreiche Nebenprodukte, die die Aufarbeitung erschweren. Verbindung 8 wird aus den genannten Gründen nur in einer Ausbeute von ca. 30 \% erhalten.

Das ${ }^{29}$ Si-NMR-Signal von Verbindung 8 liegt im Vergleich zu den ${ }^{29}$ Si-NMR-Signalen der Verbindung 3 und 7 aufgrund der zunehmenden Anzahl der Halogenatome erwartungsgemäß weiter Hochfeld:

\begin{tabular}{|l|c|c|c|}
\hline Verbindung & $\mathbf{3}$ & $\mathbf{7}$ & $\mathbf{8}$ \\
\hline Chlorfunktionen & 1 & 2 & 3 \\
\hline $\mathbf{\delta}^{29} \mathbf{S i}$ [ppm] & 16,4 & 12,3 & 5,5 \\
\hline
\end{tabular}

Tabelle 4: Vergleich der $\delta^{29}$ Si-NMR-Signale von 3, 7 und 8 


\subsubsection{Reaktionen mit Fluorsilanen}

\subsubsection{Umsetzungen mit Monofluorsilanen}

Aus der Reaktion des Fluordimethylphenylsilans mit dem lithiierten Ketazin I bzw. der lithiierten monosilylierten Verbindung 1 entstehen bei einer Temperatur von $-60{ }^{\circ} \mathrm{C}$ die neuen Verbindungen $\mathbf{9}$ und $\mathbf{1 0}$ nach der folgenden Gleichung:<smiles>[R]C/C(=N/N=C(\C)C(C)(C)C)C(C)(C)C</smiles>

I bzw. 1<smiles>[R]C/C(=N/N=C(\C[Si](C)(C)c1ccccc1)C(C)(C)C)C(C)(C)C</smiles>

9,10

\begin{tabular}{|c|c|c|}
\hline Verb. & 9 & 10 \\
\hline $\mathbf{R}$ & $\mathrm{H}$ & $\mathrm{SiMe}_{3}$ \\
\hline
\end{tabular}

Gleichung 23: Umsetzung von I bzw. 1 mit Fluordimethylphenylsilan

Die Verbindungen 9 und 10 sind gelbe Flüssigkeiten, die je in einer Ausbeute von ca. $50 \%$ entstehen. Beide Verbindungen sind unzersetzt destillierbar und bei Aufbewahrung unter Inertgas über mehrere Wochen und Monate haltbar. Die Verschiebung der $\mathrm{SiPhMe}_{2}$-Gruppe beider Verbindungen liegt im ${ }^{29} \mathrm{Si}-\mathrm{NMR}$-Spektrum bei -4 ppm.

\subsubsection{Synthese von isomeren cyclischen Silylketazinen}

Versetzt man das in $n$-Hexan lithiierte Bis(tert-butyl-methyl)ketazin bei $0{ }^{\circ} \mathrm{C}$ mit $\mathrm{Di}$ tert-butyldifluorsilan, erhält man ein Gemisch der isomeren cyclischen Ketazine 11 und 12. Wird dagegen das Di-tert-butyldifluorsilan vor dem Zutropfen in Tetrahydrofuran gelöst, isoliert man ausschließlich die Verbindung 12 (siehe Schema $5)$. 


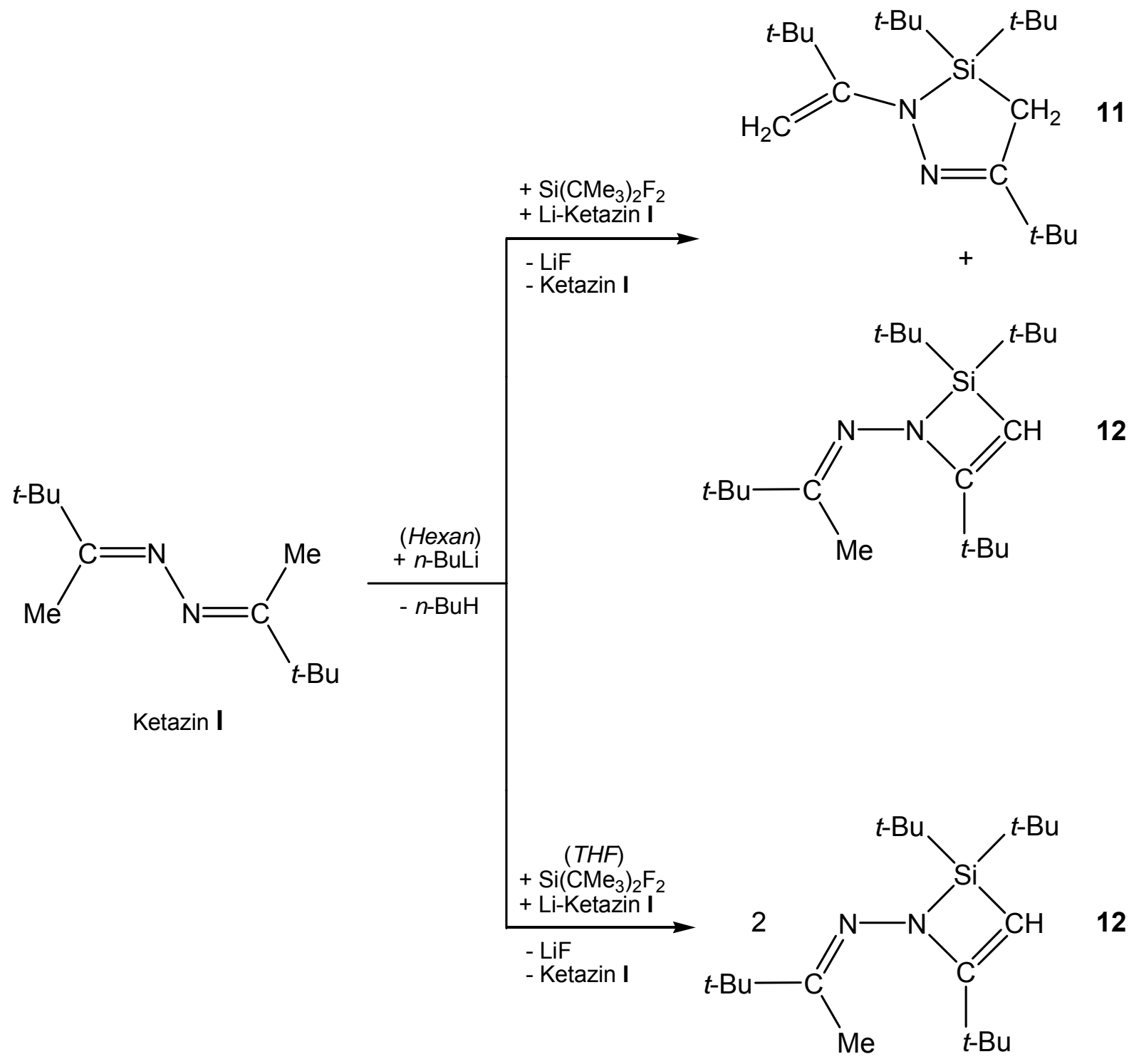

Schema 5: Darstellung von cyclischen Silylketazinen

Die Verbindungen 11 und 12 sind offensichtlich durch Eliminierung von zwei Äquivalenten Lithiumfluorid entstanden. Bei der Umsetzung des Monolithiumsalzes mit Chlorsilanen konnte ein solches Reaktionsverhalten nur als Nebenreaktion bei der Entstehung von Verbindung 2 beobachtet werden.

Aus den NMR-Daten sind beide Strukturisomere eindeutig identifizierbar:

Für Verbindung 11 kann man durch Auswertung der Integrale im ${ }^{1} \mathrm{H}-\mathrm{NMR}$-Spektrum eine gesättigte $\mathrm{CH}_{2}$-Gruppe bei 1,46 ppm und jeweils ein Signal für jedes $\mathrm{H}$-Atom der ungesättigten $=\mathrm{CH}_{2}$ bei 4,26 ppm und 4,27 ppm finden.

Hingegen weist das Spektrum von Verbindung 12 eine Methylgruppe bei 1,70 ppm und eine ungesättigte $=\mathrm{CH}$-Gruppierung mit Kopplungen von ${ }^{2} \mathrm{~J}_{1_{1}{ }^{29} \mathrm{Si}}=12,2 \mathrm{~Hz}$ und ${ }^{3} \mathrm{~J}_{{ }_{1}{ }^{15} \mathrm{~N}}=8,2 \mathrm{~Hz}$ auf. 
Das ${ }^{13}$ C-NMR-Spektrum von Verbindung 12 zeigt für die ungesättigte $=\mathrm{CH}$ Gruppierung zusätzlich eine ${ }^{1} \mathrm{~J}_{13 \mathrm{C}}{ }^{29} \mathrm{Si}-$ Kopplung von $62,3 \mathrm{~Hz}$, die im ${ }^{29} \mathrm{Si}-\mathrm{NMR}$ Spektrum wiederzufinden ist.

Anhand der ${ }^{1} \mathrm{H}$ - und ${ }^{13} \mathrm{C}-N M R-D a t e n$ kann Verbindung 12 auch mit dem strukturisomeren Silaethen beschrieben werden:

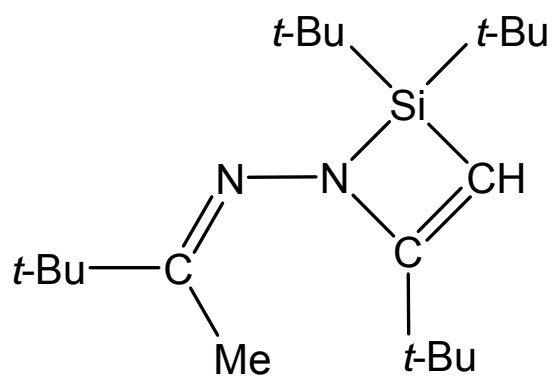

12<smiles>CCCCC(C)(C)C(C)(C)C</smiles>

Silaethen

Abbildung 8: Vergleich der Verbindung 12 mit dem hypothetischen isomeren Silaethen

Die sehr unterschiedlichen Signale für die Stickstoffatome im ${ }^{15} \mathrm{~N}-\mathrm{NMR}$-Spektrum von - 232,5 ppm und - 39,1 ppm sprechen jedoch gegen das Silaethen und für den Strukturvorschlag von Verbindung 12. Die NMR-Daten des Siliciums lassen mit einer Verschiebung von 33,3 ppm im ${ }^{29}$ Si-NMR-Spektrum keine eindeutigen Rückschlüsse zu. Das freie Elektronenpaar des Stickstoffs könnte durch die Ausbildung einer Adduktbindung zum Silicium die ${ }^{29}$ Si-Verschiebung in Richtung Hochfeld beeinflussen. Zudem weisen auch die stabilen Silaethene von BROOK ${ }^{[59]}$ und WIBERG ${ }^{[60]}$ stark unterschiedliche ${ }^{13} \mathrm{C}$ - und ${ }^{29} \mathrm{Si}-$ Verschiebungen für die $\mathrm{Si}=\mathrm{C}$ Doppelbindung auf:<smiles>C[SiH2]C([14CH3])=[Si]([SiH3])[SiH2]C</smiles><smiles>CCCCCC([AsH3])=[Si](C)C</smiles>

$\delta^{13} \mathrm{C}=214,2 \mathrm{ppm}$
$\delta^{29} \mathrm{Si}=41,4 \mathrm{ppm}$

$\delta{ }^{13} \mathrm{C}=77,2 \mathrm{ppm}$ $\delta^{29} \mathrm{Si}=144,2 \mathrm{ppm}$ 
WIBERG konnte zeigen, dass Silaethene mit einer Reihe von neutralen und anionischen Lewis-Basen, wie z. B. THF, NMe ${ }_{3}$, Pyridin und $\mathrm{F}^{-}$, Addukte bilden ${ }^{[61]}$.
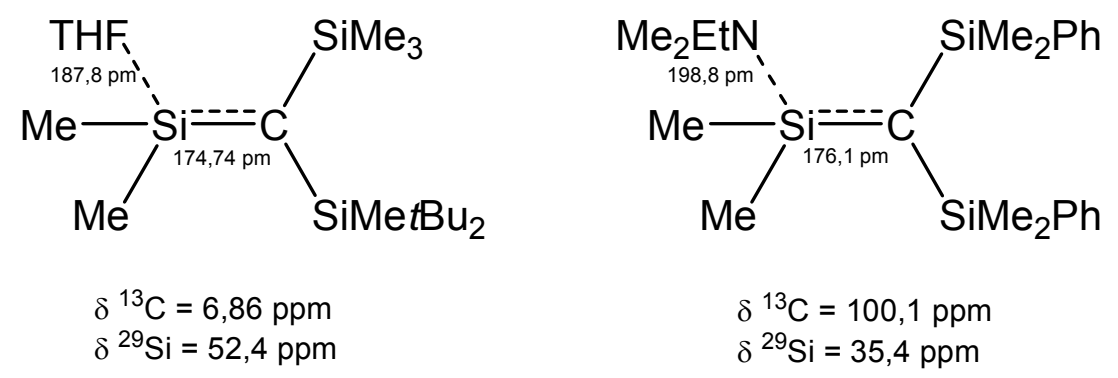

Abbildung 10: Stabile Silaethen-Addukte

Die Lewis-Basen koordinieren in diesen Addukten immer an dem ungesättigten Siliciumatom. Der Donor ist über eine vergleichsweise lange Bindung mit dem ungesättigten Siliciumatom verknüpft. Die Addition der Base ist mit einer Vergrößerung des Si=C-Abstandes verbunden (von 170,2 pm beim Silaethen auf 174,74 pm beim Silaethen-THF-Addukt; vgl. Abbildung 9 und 10). Im Zuge der Adduktbindung bildet das zuvor planare Siliciumatom einen verzerrten Tetraeder, während das ungesättigte Kohlenstoffatom seine Planarität beibehält. Obwohl es sich bei diesen Addukten also streng genommen nicht um Verbindungen mit einem ungesättigten Silicium handelt, ist ihre Verwandtschaft mit den Silaethenen nicht von der Hand zu weisen. Die Molekülgeometrie erweckt den Eindruck, als ob die Annäherung der Donoren (THF, $\mathrm{NR}_{3}$ etc.) an das ungesättigte Siliciumatom auf der Reaktionskoordinate kurz vor Erreichen der tetraedrischen Endkonfiguration zum Stillstand gekommen wäre.

In dem zu Verbindung 12 isomeren Silaethen könnte ein freies Elektronenpaar der Ketazin-Stickstoffatome die Rolle des stabilisierenden Donors übernehmen. Dafür spräche die ${ }^{29}$ Si-Verschiebung von 12. Sie ähnelt mit 33,3 ppm der Verschiebung des von WIBERG ${ }^{[62]}$ isolierten Silaethen-Aminadduktes $\left(\delta^{29} \mathrm{Si}=35,4 \mathrm{ppm}\right)$. Eine Resonanzstruktur von 12, die auch die zwitterionische Natur der Si=C-Doppelbindung berücksichtigt, könnte deshalb in Ergänzung zu Abbildung 8 folgendermaßen beschrieben werden: 
<smiles>CC(=NN=C(C=[Si](C(C)(C)C)C(C)(C)C)C(C)(C)C)C(C)(C)C</smiles>

Silaethen<smiles>CC(=NN1C(C(C)(C)C)=C[Si]1(C(C)(C)C)C(C)(C)C)C(C)(C)C</smiles>

12

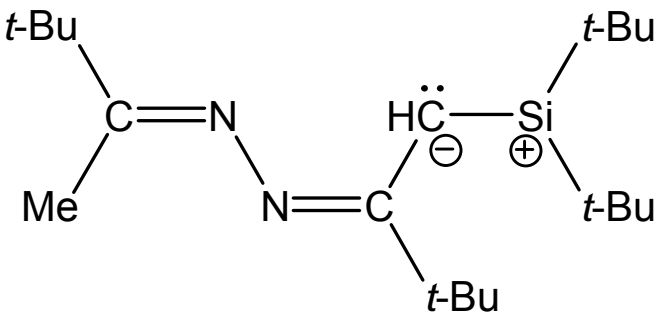

zwitterionische Struktur

$$
\uparrow
$$<smiles></smiles>

donor-stabilisiert

Schema 6: Mögliche Resonanzstrukturen von Verbindung 12

Die Produktbildung von Verbindung 11 und 12 wirft die Frage auf, wie aus den Monolithiumsalzen des Ketazins I zwei Lithiumfluorid eliminiert werden. Aus dem Arbeitskreis MELLER ${ }^{[9]}$ ist bekannt, dass bei der Umsetzung von dilithiierten Ketazinen mit Trihalogenboranen ein Gemisch aus drei verschiedenen Produkten entsteht: 


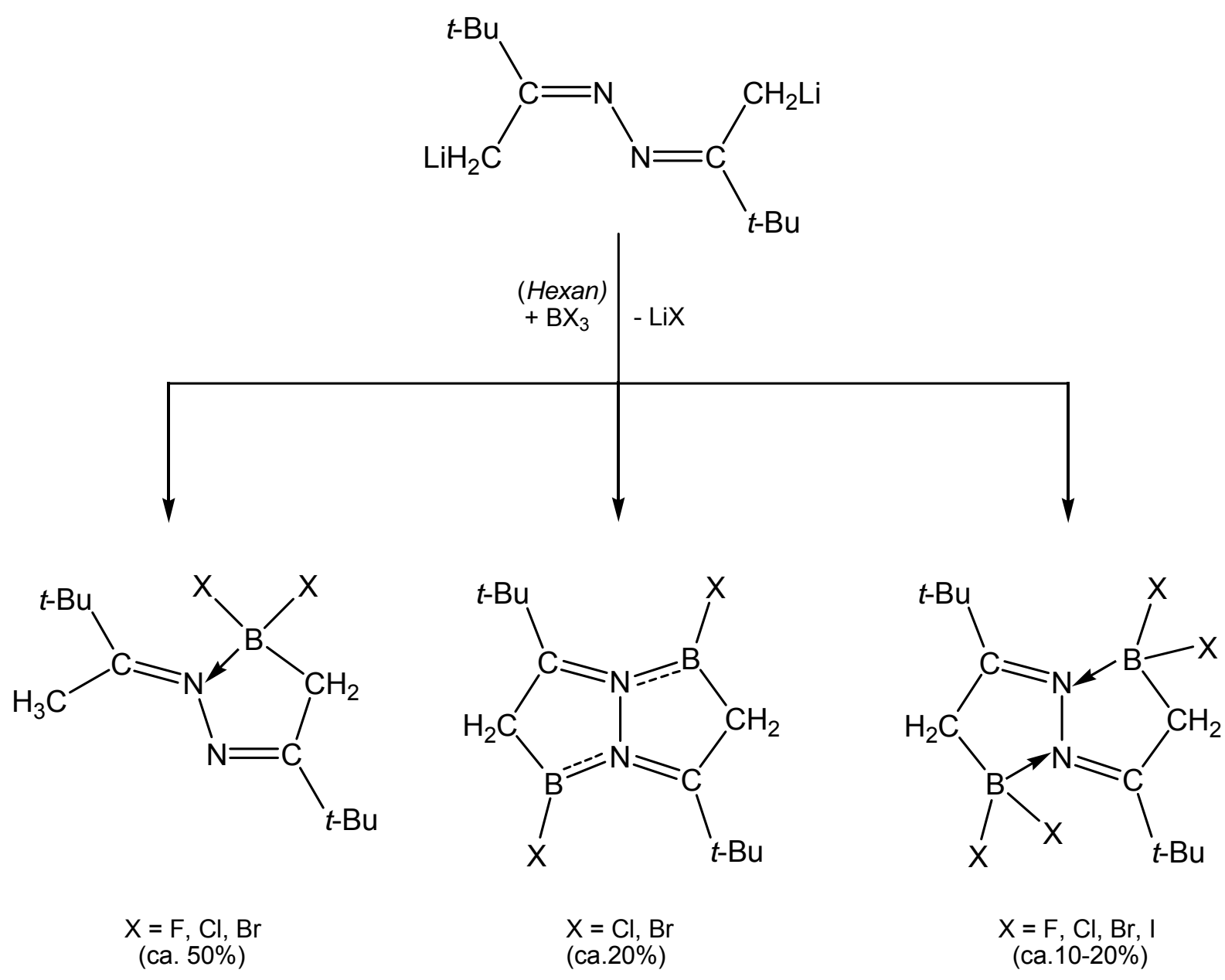

Schema 7: Reaktionen des dilithiierten Ketazins I mit Trihalogenboranen

Eine mögliche Erklärung für die Bildung der gefundenen Produkte ist eine intermolekulare Umlithiierung. Auch die Rückgewinnung von Ausgangssubstanz spricht für einen solchen Mechanismus.

Bei den Hydrazonen sind Umlithiierungsreaktionen im Arbeitskreis KLINGEBIEL ${ }^{[63,64]}$ beschrieben worden: 
<smiles>[R2]/C(C)=N/N([R7])Cl</smiles>

Hydrazon

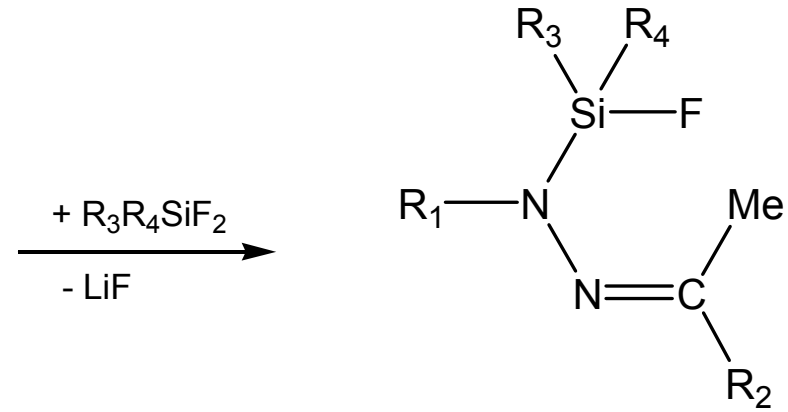

+ Li-Hydrazon $\mid \begin{aligned} & \text { - Hydrazon } \\ & - \text { LiF }\end{aligned}$<smiles>[R2]C1=NN([R])[Si]([R3])([R3])C1</smiles>

Diazasilacyclopenten

Schema 8: Umlithiierung bei Hydrazonen

Ein ähnlicher Mechanismus wäre auch für die Ketazine denkbar. Allerdings sprechen die isolierten mono- und disilylierten ${ }^{[65]}$ acyclischen Produkte dafür, dass das Alkalimetall nicht wie erwartet am Stickstoff, sondern am a-Kohlenstoff koordiniert ist. Die Forschungsergebnisse mit Halogenboranen untermauern diese These (siehe Schema 7). Die Lewistruktur des monolithiierten Bis(tert-butyl-methyl)ketazins könnte folgendermaßen aussehen:<smiles>CCN(N=C(C)C(C)(C)C)C(C)(C)C</smiles>

Abbildung 11: Strukturvorschlag für das Monolithiumsalz des Bis(tert-butyl-methyl)ketazins 
Eine Alkyllithium-Verbindung ist zwar kovalent, aber durch das elektropositive Metall stark polarisiert. Das Metall bildet das positive Ende des Dipols. Der ionische Anteil der Kohlenstoff-Lithium-Bindung liegt nach VoLLHARDT ${ }^{[66]}$ bei etwa $40 \%$. Nach VON SCHLEYER ${ }^{[67]}$ sogar bei mehr als $90 \%$. Unter Berücksichtigung dieser kovalenten und ionischen Anteile und der Enamin-Imin-Tautomerie von Carbonylverbindungen könnte man das Monolithiumsalz eines Ketazins auch wie folgt beschreiben:<smiles>CC(=NN=C(C)C(C)(C)C)C(C)(C)C</smiles>

Imin-kovalent<smiles>CC1([V])CCCCC1</smiles><smiles>C=C(N(Cl)/N=C(\C)C(C)(C)C)C(C)(C)C</smiles>

Enamin-kovalent<smiles>CC(=NN=C(C)C(C)(C)C)C(C)(C)C</smiles>

Imin-ionisch

$$
\uparrow
$$<smiles>C=C(N=C(C)C(C)(C)C)C(C)(C)C</smiles>

Enamin-ionisch<smiles>C#CC(=C)/N=C(\C)[18O]</smiles>

Schema 9: Mögliche Resonanzstrukturen des Monolithiumsalzes

Aus pragmatischen Gründen und der Übersichtlichkeit halber wird das Lithiumsalz im Folgenden in der Iminform abgebildet. Bei Ketazinen steht ein Strukturbeweis im Festkörper durch eine Röntgenanalyse noch aus. Bei Hydrazonen werden Li-NKoordinationen und Oligomerisationen der Verbindungen im Kristall gefunden. 
Für die Umlithiierung und die Bildung von Diazasilacyclopentenen lassen sich drei mögliche Mechanismen formulieren, wobei die beiden Reaktionswege $A$ und $B$ davon ausgehen, dass zuerst eine Substitution des Dihalogensilans unter Abspaltung von Lithiumfluorid am a-Kohlenstoff des monolithiierten Ketazins stattfindet. Dies wird durch die bisher beschrieben acyclischen Silylketazine, z. B. Verbindung 2 und 3, bekräftigt.

Reaktionsweg $C$ greift die von den Hydrazonen bekannte Erstsubstitution am Stickstoff auf (vgl. Schema 8).

Im Verlauf von Reaktionsweg $A$ kommt es anschließend zu einer intermolekularen Umlagerung, bei der sich das Lithiumion an das $\alpha$-Kohlenstoffatom der Methylgruppe anlagert. Das durch die LiHal-Abspaltung entstehende Carbanion stabilisiert sich durch Ausbildung einer $\mathrm{C}=\mathrm{C}$-Doppelbindung und Auflösung der benachbarten $\mathrm{C}=\mathrm{N}$ Doppelbindung. Es kommt zu einer intramolekularen Cyclisierung durch den nukleophilen Angriff des freiwerdenden Elektronenpaars auf das Siliciumatom und ein Diazasilacyclopenten entsteht (siehe Schema 10).

In Reaktionsweg $B$ dagegen bindet das Lithiumion an die silylsubstituierte- $\mathrm{CH}_{2}$ Gruppierung. Nach Eliminierung des Lithiumhalogenids bildet sich intermediär ein Silaethen, welches sich durch intramolekulare Protonenwanderung zum Diazasilacyclopenten stabilisiert (siehe Schema 11).

In Reaktionsweg $C$ erfolgt die Umlithiierung am a-Kohlenstoff der Methylgruppe und durch Salzeliminierung entsteht das Diazasilacyclopenten (siehe Schema 12). 

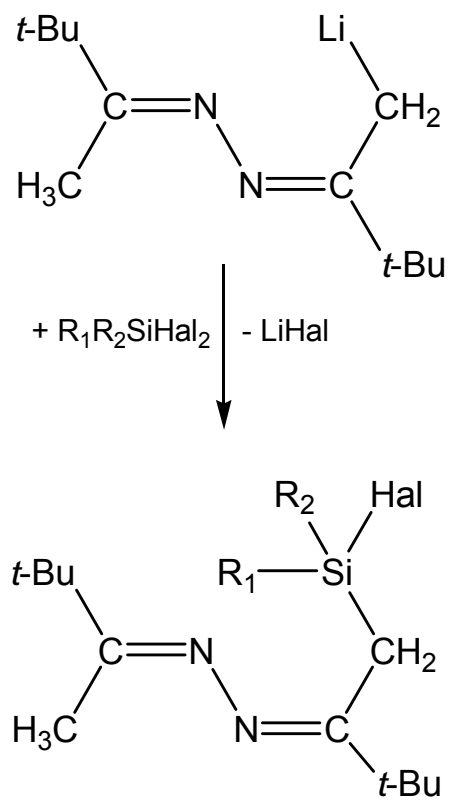

+ Li-Ketazin $\rfloor$ - Ketazin

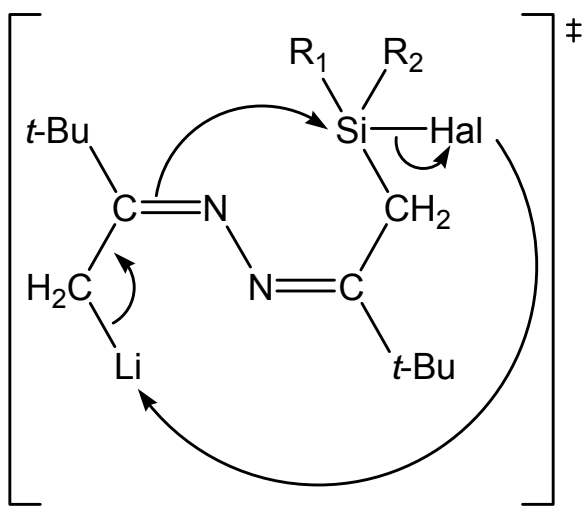

- LiHal<smiles>[R][Si]1([R])CC(C(C)(C)C)=NN1C(=C)C(C)(C)C</smiles>

Diazasilacyclopenten

Schema 10: Reaktionsweg $A$ 


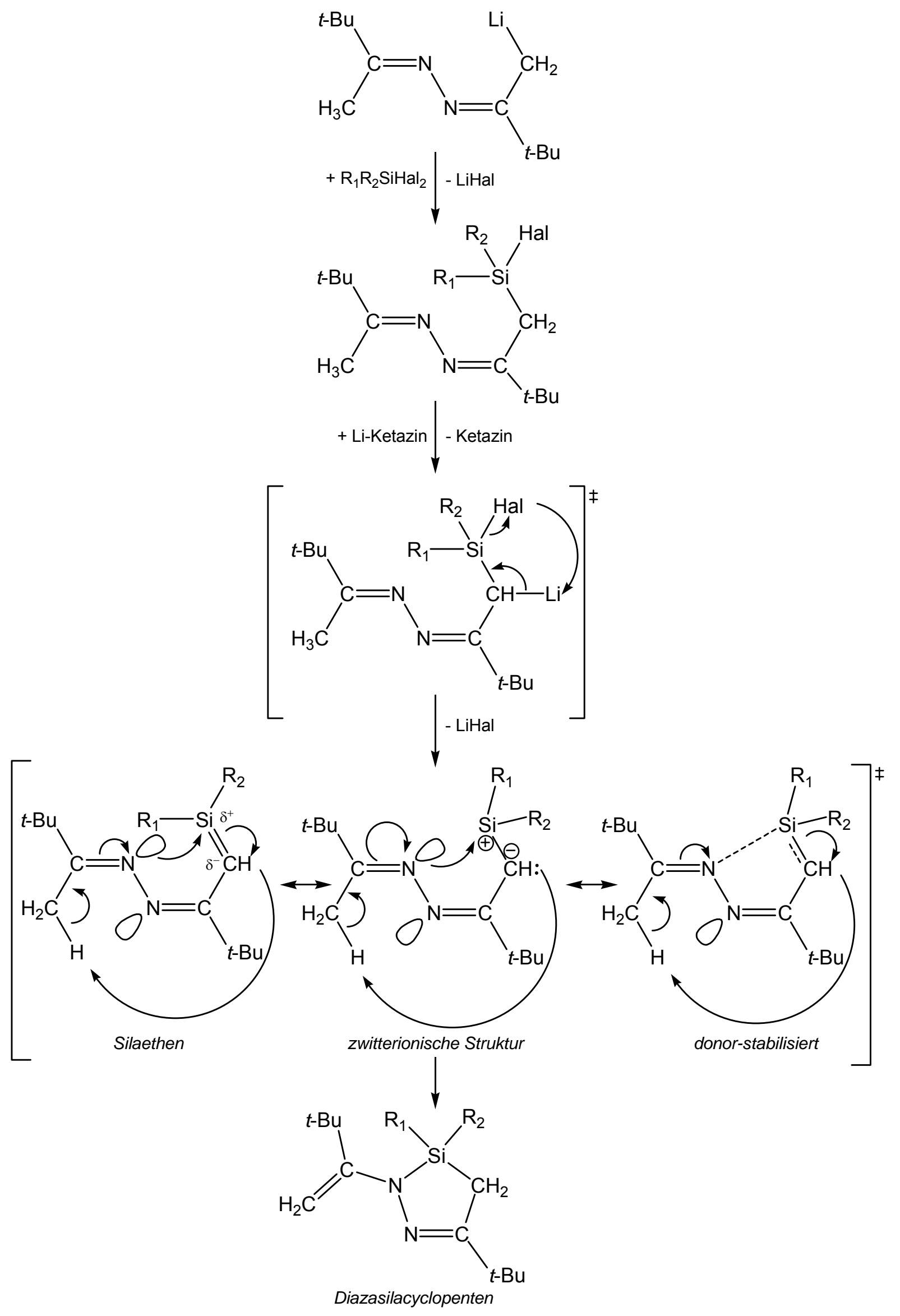

Schema 11: Reaktionsweg $B$ 

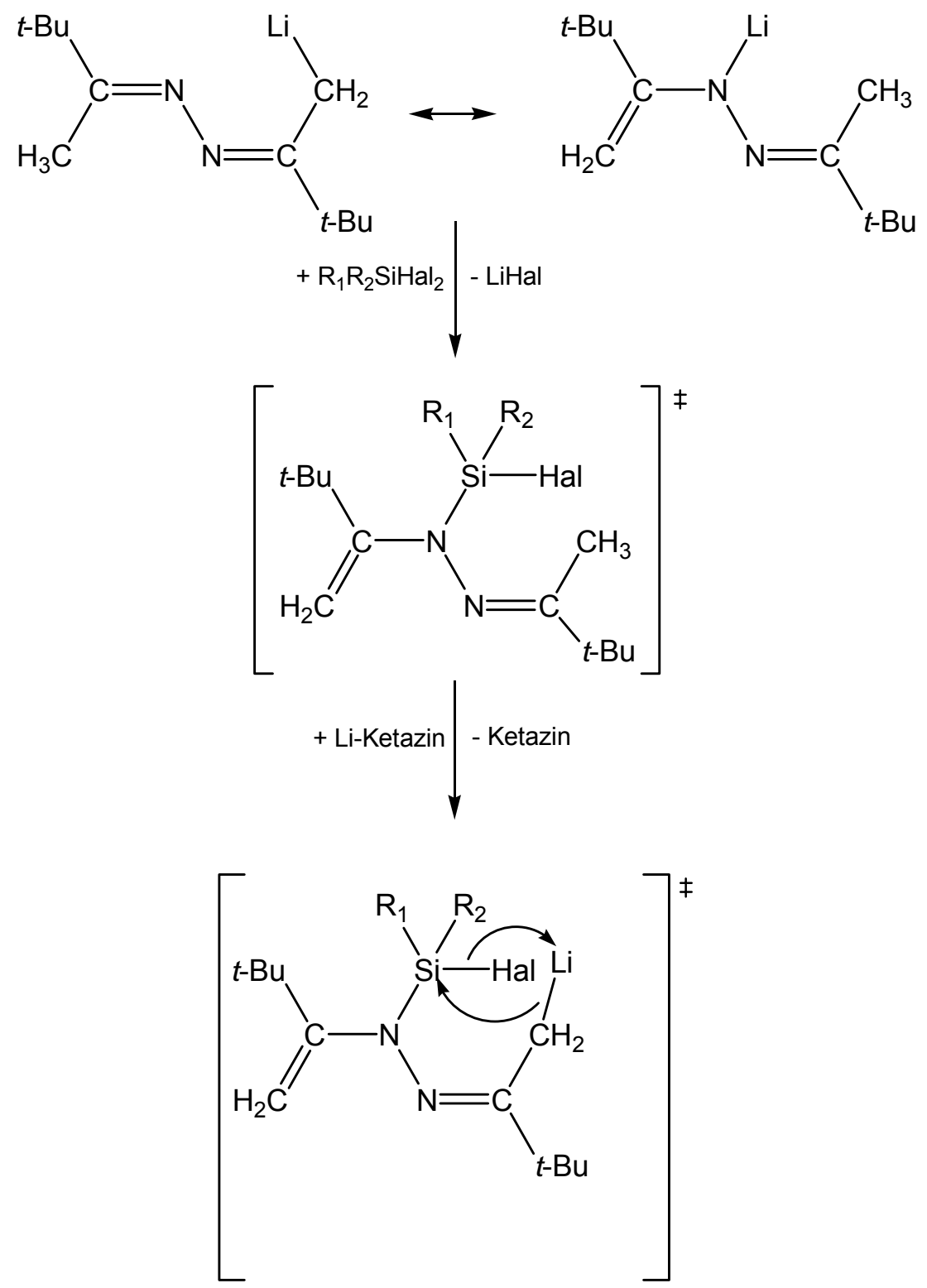

- LiHal<smiles>[R15]C(=C)N1N=C([R15])C[Si]1([R])[R]</smiles>

Diazasilacyclopenten

Schema 12: Reaktionsweg C 
Isolierte Disubstitutionsprodukte ${ }^{[10,65]}$ des Ketazins I lassen auf ein analog dem Monolithiumsalz aufgebautes Dilithiumsalz schließen. Bei einer Umlithiierung sollte das Lithiumion entsprechend Reaktionsweg $A$ also am $\alpha$-Kohlenstoffatom der Methylgruppe gebunden werden. Zusätzlich könnten sterisch anspruchsvolle Silylsubstituenten die $\mathrm{CH}_{2}$-Gruppierung abschirmen und eine Deprotonierung der Methylengruppe behindern.

Reaktionsweg $C$ berücksichtigt die Tatsache, das in der Silicium-organischen Chemie die Enamin-Form des Lithiumsalzes stabiler ist als die Imin-Form ${ }^{[67]}$. Si-N-Bindungen werden gegenüber Si-C-Bindungen bevorzugt gebildet. Allerdings konnte bisher kein Monosubstitutionsprodukt eines Ketazins mit Enamin-Struktur synthetisiert werden. Erklären ließe sich dies durch die sterisch anspruchsvolle tert-Butylgruppe in Nachbarschaft. Sie könnte eine Silylgruppenwanderung bewirken, durch die sich das Monosubstitutionsprodukt stabilisiert.

Für den Mechanismus $B$ spricht, dass sich der - I-Effekt der Halogenatome auch auf die Acidität der Protonen der Methylengruppierung auswirkt. Diese werden elektronenärmer, was sich durch den Vergleich der ${ }^{1} \mathrm{H}-\mathrm{NMR}$-Spektren der folgenden silylsubstituierten acyclischen Ketazine belegen lässt:

\begin{tabular}{|l|c|c|c|c|c|}
\hline Verbindung & $\mathbf{1}$ & $\mathbf{2}$ & $\mathbf{3}$ & $\mathbf{7}$ & $\mathbf{8}$ \\
\hline $\mathbf{\delta}^{\mathbf{1}} \mathbf{H}$ [ppm] & 1,91 & 2,25 & 2,55 & 2,97 & 3,03 \\
\hline
\end{tabular}

Tabelle 5: Vergleich $\delta{ }^{1} \mathrm{H}$-Signale der Methylengruppe von 1, 2, 3, 7, 8

Die Lithiumhalogenid-Eliminierung würde zudem durch die räumliche Nähe des Lithiumions zum Halogenid begünstigt. Ein zusätzliches Indiz liefert die Tatsache, dass bei den Hydrazonen aus Diazasilacylopentenen bereits Produkte synthetisiert werden konnten, deren Entstehung über intermediäre Silaethene verläuft $^{[48,49,50,52,53,63,68]}$, z. B.: 

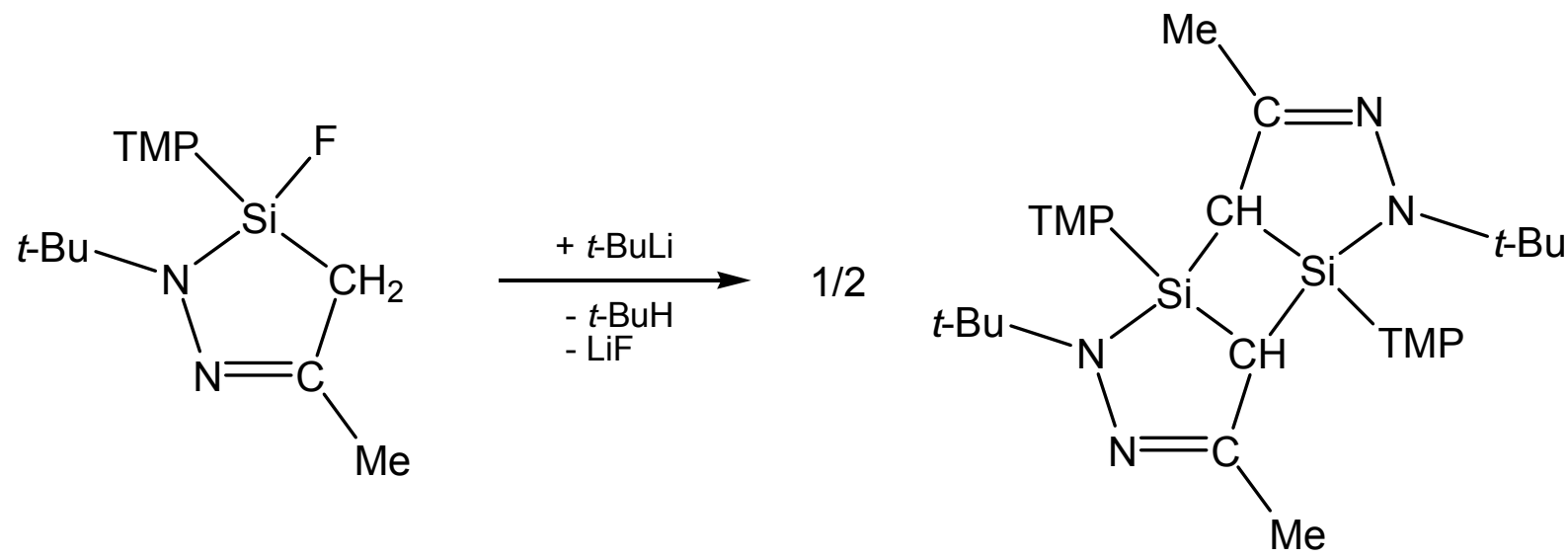

Gleichung 24: Dimerisation eines intermediäres Silaethens zu einem Tricyclus

Die Entstehung von Verbindung 11 und 12 lässt sich über Reaktionsweg $B$ erklären: Das postulierte intermediäre Silaethen könnte sich über beide freien Elektronenpaare der Ketazin-Stickstoffatome Donor-stabilisieren. Welches freie Elektronenpaar letztendlich eine Adduktbindung eingeht, hängt vom sterischen Anspruch der Silylsubstituenten und vom Lösungsmittel ab. Durch Zugabe der Lewis-Base THF würde das Silaethen schon durch den Ether stabilisiert. Nach WIBERG ${ }^{[62]}$ ändert sich dadurch die Geometrie am Siliciumatom des intermediären Silaethens und somit die räumliche Ausrichtung zu den freien Elektronenpaaren der Ketazin-Stickstoffatome. Die Entstehung des viergliedrigen Ringes wird dann gegenüber dem energetisch günstigeren Diazasilacyclopenten favorisiert. 


\subsubsection{Ein acyclisches Fluorsilylketazin}

Durch Umsetzung des monolithiierten Bis(tert-butyl-methyl)ketazins I mit einem Äquivalent $N$-(Trifluorsilyl)-2-methyl- $N$-(trimethylsilyl)propan-2-amin bei $-60^{\circ} \mathrm{C}$, konnte das 2-(1-(tert-Butyl-(trimethylsilyl)amino-difluorosilyl)-3,3-dimethylbutan-2yliden)-1-(3,3-dimethylbutan-2-yliden)hydrazin 13 isoliert werden:
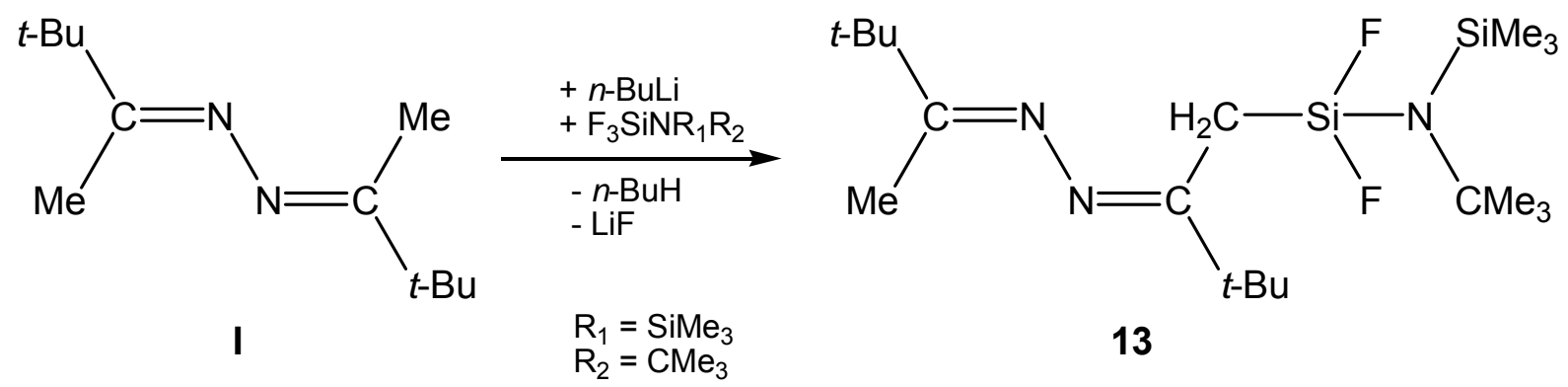

Gleichung 25: Darstellung eines acyclischen Fluorsilylketazins

Verbindung 13 ist eine hellgelber Feststoff, der bei der Destillation aus der flüssigen gelben Vorlage erstarrt.

Das Signal der $\mathrm{CH}_{2}$-Gruppierung bei 2,23 ppm spaltet im ${ }^{1} \mathrm{H}-\mathrm{NMR}$ zu einem AA'XX'Spektrum auf, welches sich im ${ }^{19} \mathrm{~F}-\mathrm{NMR}$ wiederfindet. Die $\mathrm{SiF}_{2} \mathrm{~N}-\mathrm{Gruppe}$ zeigt im ${ }^{29}$ Si-NMR-Spektrum ein Triplett bei - 40,2 ppm mit einer ${ }^{1} \mathrm{~J}_{\mathrm{SiF}}-K o p p l u n g$ von $283 \mathrm{~Hz}$.

Durch Umkristallisation dieser Verbindung in $n$-Hexan konnten Kristalle erhalten werden, die zur Röntgenstrukturanalyse geeignet waren. Die Struktur ist in folgender Abbildung wiedergegeben: 


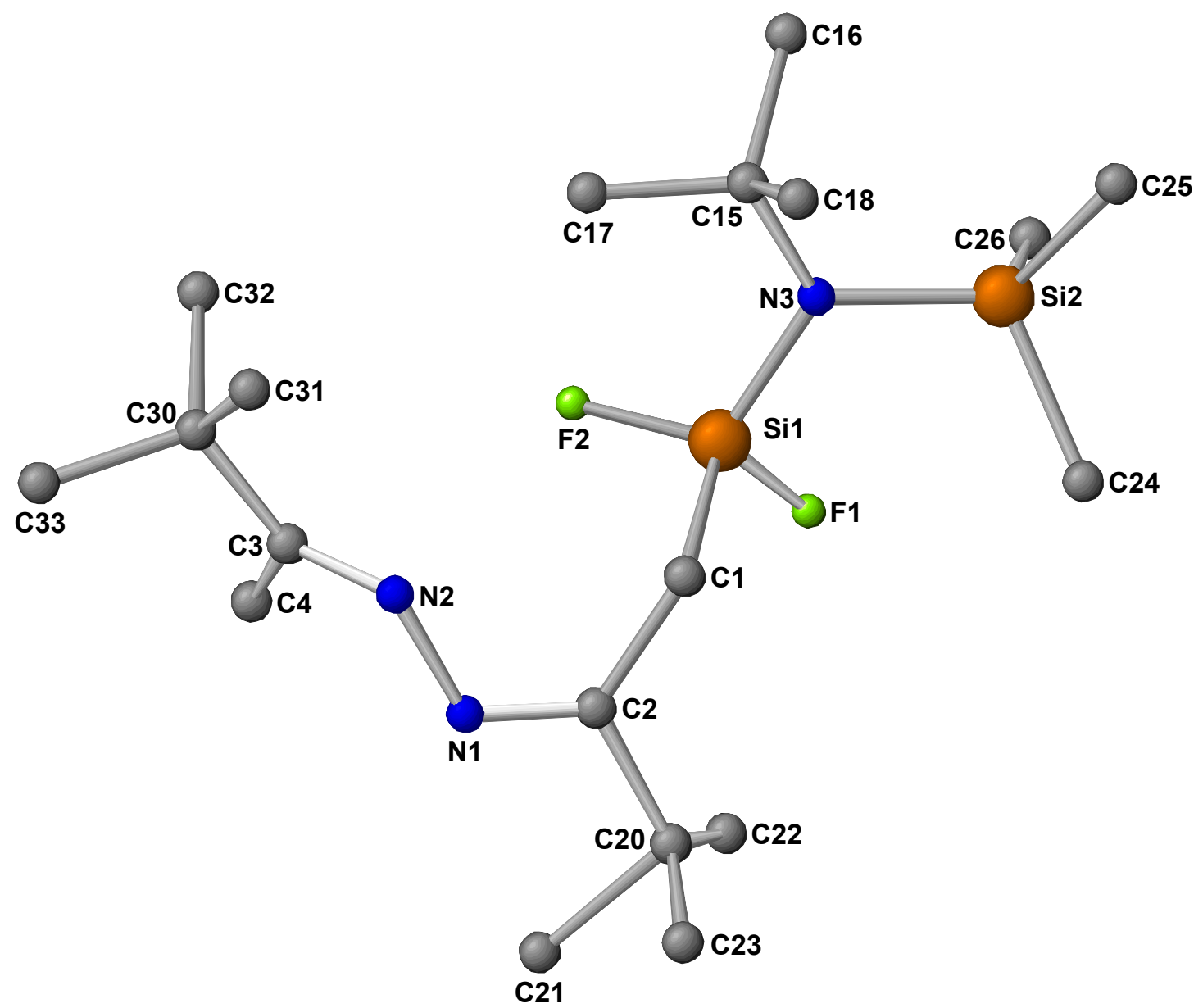

Abbildung 12: Kristallstruktur von Verbindung 13

\begin{tabular}{|l|r|}
\hline C1-C2 & $152,5 \mathrm{pm}$ \\
\hline C2-N1 & $123,5 \mathrm{pm}$ \\
\hline N1-N2 & $141,7 \mathrm{pm}$ \\
\hline N2-C3 & $130,8 \mathrm{pm}$ \\
\hline C3-C4 & $150,3 \mathrm{pm}$ \\
\hline C1-Si1 & $186,1 \mathrm{pm}$ \\
\hline Si1-F1 & $159,5 \mathrm{pm}$ \\
\hline
\end{tabular}

\begin{tabular}{|l|r|}
\hline Si1-F2 & $158,8 \mathrm{pm}$ \\
\hline Si1-N3 & $170,3 \mathrm{pm}$ \\
\hline N3-C15 & $152,0 \mathrm{pm}$ \\
\hline N3-Si2 & $178,5 \mathrm{pm}$ \\
\hline C2-C20 & $153,6 \mathrm{pm}$ \\
\hline C3-C30 & $150,6 \mathrm{pm}$ \\
\hline
\end{tabular}

Tabelle 6: Ausgewählte Bindungslängen von Verbindung 13

Verbindung 13 bildet ein monoklines Kristallsystem mit der Raumgruppe P2(1)/c. Die Ketazinstruktur bleibt erhalten, ist aber über das C3-N2-N1-C2-Gerüst um 126,3 tordiert. Die CNN-Winkel sind mit $115,8^{\circ}$ und $118,2^{\circ}$ aufgrund des freien Elektronenpaares etwas kleiner als der ideale Winkel eines $\mathrm{sp}^{2}$-hybridisierten Stickstoffatoms. Die C=N-Doppelbindungslängen sind unterschiedlich. Die 
N2-C3-Bindung liegt mit 130,8 pm im typischen Bereich, während die C2-N1-Bindung mit 123,5 pm um 7,3 pm kürzer ist. Die Geometrie am Si1-Atom ist mit einer Winkelsumme von $655,5^{\circ}$ tetraedrisch. Die Si-F-Bindungen betragen beide rund 159 pm.

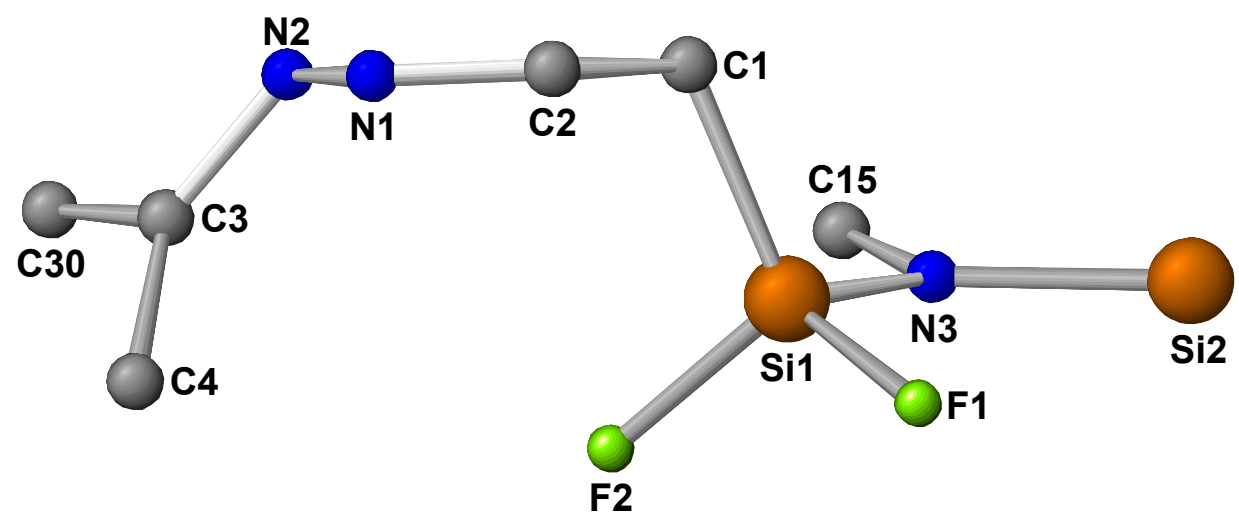

Abbildung 13: Seitlicher Blick auf die N2-N1-C2-C1-Ebene von Verbindung 13

\begin{tabular}{|l|r|}
\hline C4-C3-N2 & $121,1^{\circ}$ \\
\hline C3-N2-N1 & $115,8^{\circ}$ \\
\hline N2-N1-C2 & $118,2^{\circ}$ \\
\hline N1-C2-C1 & $123,5^{\circ}$ \\
\hline C2-C1-Si1 & $119,3^{\circ}$ \\
\hline C1-Si1-N3 & $115,0^{\circ}$ \\
\hline Si1-N3-C15 & $120,2^{\circ}$ \\
\hline Si1-N3-Si2 & $117,3^{\circ}$ \\
\hline
\end{tabular}

\begin{tabular}{|l|r|}
\hline C1-Si1-F2 & $108,4^{\circ}$ \\
\hline C1-Si1-F1 & $109,8^{\circ}$ \\
\hline Si1-N3-C15 & $120,2^{\circ}$ \\
\hline Si1-N3-Si2 & $117,3^{\circ}$ \\
\hline Si2-N3-C15 & $122,1^{\circ}$ \\
\hline F1-Si1-F2 & $101,9^{\circ}$ \\
\hline F2-Si1-N3 & $114,8^{\circ}$ \\
\hline F1-Si1-N3 & $106,0^{\circ}$ \\
\hline
\end{tabular}

Tabelle 7: Ausgewählte Bindungswinkel von Verbindung 13 


\subsubsection{Reaktion mit Tetrafluorsilan}

Das Lithiumderivat des tert-Butylmethylhydrazons reagiert mit Tetrafluorsilan im Molverhältnis 4 : 1 zum Tetrakis(hydrazonyl)silan ${ }^{[47]}$ :<smiles>CC(=NC(C)(C)C)NCl</smiles>

Gleichung 26: Darstellung eines Tetrakis(hydrazonyl)silans

Bei der Reaktion der monolithiierten Verbindung I mit Tetrafluorsilan im Verhältnis 2:1 konnte das erwartete Bis(ketazinyl)silan nicht isoliert werden. Das Tris(ketazinyl)silan 14 hingegen ist stabil und hat einen Siedepunkt von $124{ }^{\circ} \mathrm{C}$ bei 0,04 mbar.

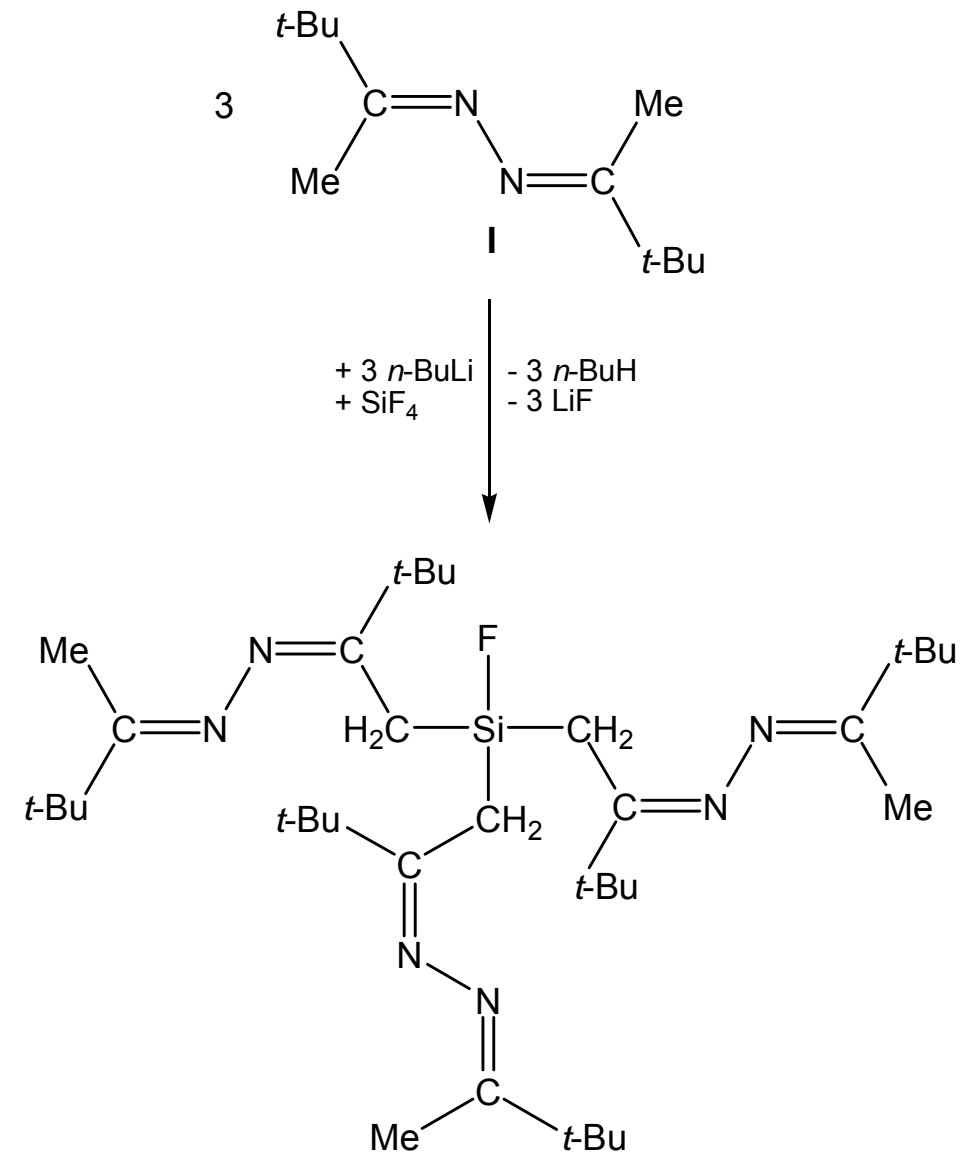


Das Tris(2-(2-(3,3-dimethylbutan-2-yliden)hydrazono)-3,3-dimethylbut-1-yl)-fluorsilan 14 ist eine gelbe Flüssigkeit.

Das ${ }^{1} \mathrm{H}-\mathrm{NMR}$-Spektrum zeigt bei 2,22 ppm ein Dublett mit einer ${ }^{3} \mathrm{~J}_{\mathrm{HF}}-$ Kopplung von 7,8 Hz. Diese Kopplung findet man im ${ }^{19} \mathrm{~F}-\mathrm{NMR}$ wieder. Die ${ }^{29} \mathrm{Si}-$ Verschiebung liegt bei 14,9 ppm und die ${ }^{1} \mathrm{~J}_{\mathrm{SiF}_{\mathrm{F}}}$-Kopplung beträgt $308 \mathrm{~Hz}$. 


\subsection{Umsetzungen des dilithiierten Ketazins}

\subsubsection{Reaktionen mit Chlorsilanen}

\subsubsection{Reaktionen mit Dichlorsilanen}

Setzt man das dilithierte Ketazin I mit einem Äquivalent Dichlordimethylsilan um, erhält man, abhängig von den Reaktionsbedingungen, die Verbindungen 15 und 16:

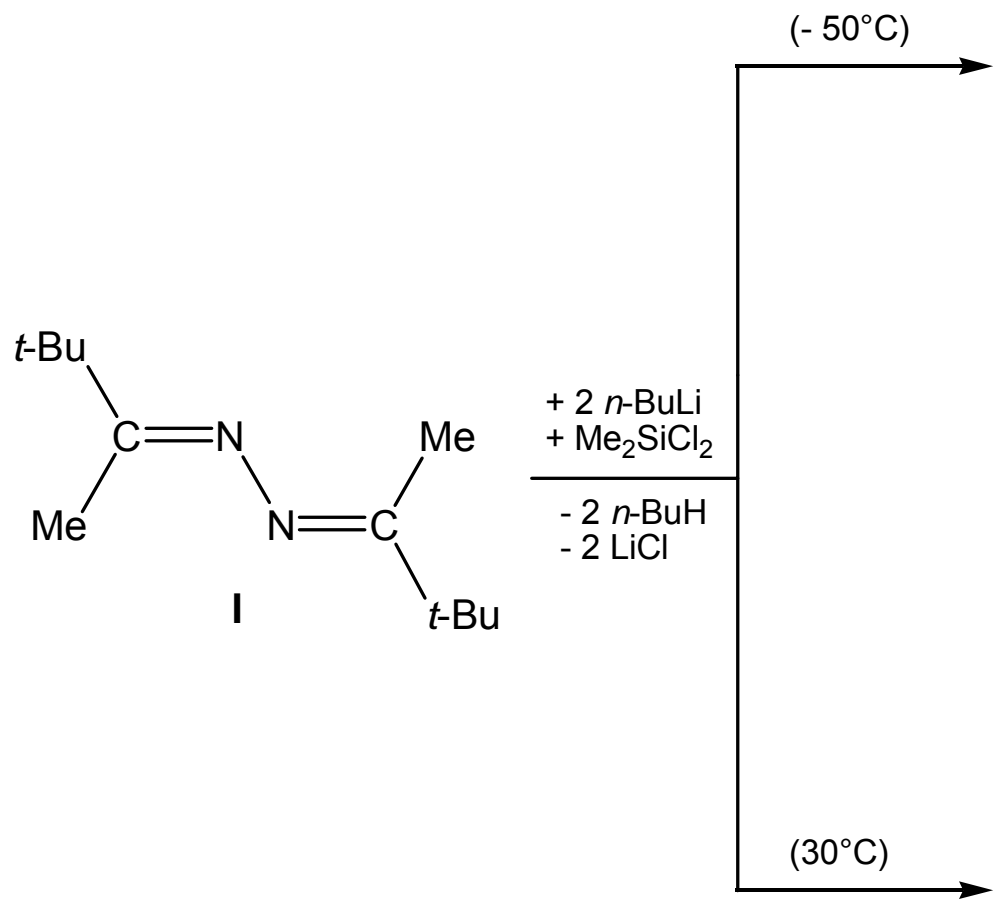<smiles>C=C(N1N=C(C(C)(C)C)C[Si]1(C)C)C(C)(C)C</smiles><smiles>CC(C)=NN1C(Cl)(C(C)(C)C)C[Si]1(C)C</smiles>

16

Gleichung 28: Umsetzung des Dilithiumsalzes mit Dichlordimethylsilan

Tropft man das Dilithiumsalz bei $-50{ }^{\circ} \mathrm{C}$ hinzu, erhält man das 5-tert-Butyl-3,4dihydro-3,3-dimethyl-2-(3,3-dimethylbut-1-en-2-yl)-2H-1,2,3-diazasilol 15 in einer Ausbeute von ca. $60 \%$ als hellgelbe Flüssigkeit. Verbindung 15 ist über einen Zeitraum von mehreren Wochen unter Inertgas lagerfähig. 
Das N-(4-tert-Butyl-4-chlor-2,2-dimethyl-1,2-azasiletidin-1-yl)-3,3-dimethylbutan-2imin 16 wird dagegen als Nebenprodukt bei einer Reaktionstemperatur von $30^{\circ} \mathrm{C}$ gebildet. Die Entstehung des Imins 16 lässt sich durch eine unvollständige Lithiierung des Bis(tert-butyl-methyl)ketazins erklären: Nach dem selben Mechanismus wie bei Verbindung 12 (siehe Schema 11, Seite 35) bildet sich ein viergliedriger Ring unter Abspaltung eines Moleküls Chlorwasserstoff. Da Ketazine, wie bereits in Kapitel 1.1.4 beschrieben, mit Mineralsäuren stabile Aziniumsalze bilden, verbleibt Chlorwasserstoff in der Reaktionslösung. Dieser wird an die Doppelbindung addiert und es entsteht das Imin 16.

Das Dilithiumderivat von Verbindung I bzw. des Bis(tert-butyl-(trimethylsilyl)methyl)ketazins II reagiert mit Dichlormethylphenylsilan zu den cyclischen Verbindungen 17 und 18:<smiles>[R]CC([R15])=NN=C([R15])C[R]</smiles>

I bzw. ||<smiles>[R]C=C(N1N=C([R15])C([R])[Si]1(C)c1ccccc1)C(C)(C)C</smiles>

17,18

\begin{tabular}{|c|c|c|}
\hline Verb. & 17 & 18 \\
\hline R & $\mathrm{H}$ & $\mathrm{SiMe}_{3}$ \\
\hline
\end{tabular}

Gleichung 29: Reaktion des Dilithiumderivates von I und II mit $\mathrm{MePhSiCl}_{2}$

Die Verbindungen 17 und 18 werden in einer Ausbeute von etwa 65 \% bzw. 40 \% erhalten. Beide Verbindungen sind gelbe Flüssigkeiten, im Hochvakuum unzersetzt destillierbar und über mehrere Wochen unter Inertgas stabil. Das 5-tert-Butyl-3,4dihydro-3-methyl-2-(3,3-dimethyl-1-(trimethylsilyl)but-1-en-2-yl)-4-(trimethylsilyl)-3phenyl-2H-1,2,3-diazasilol 18 bildet ein Isomerengemisch im Verhältnis 4:1. Der + M- und - I-Effekt des Phenylrestes bewirkt bei 17 im Vergleich zum Alkylrest der Verbindung 15 eine Verschiebung des ${ }^{29}$ Si-NMR-Signals von 19,2 ppm (Verbindung 
15) auf 9,1 ppm (Verbindung 17). Das ${ }^{29}$ Si-NMR-Signal der PhMeSi-Gruppierung von Verbindung 18 liegt bei 14,5 ppm.

\subsubsection{Dichlorfunktionelle cyclische Ketazine}

Umsetzungen der dilithiierten Verbindung I mit Trichlorboranen führten im Arbeitskreis MELLER ${ }^{[9]}$ zur Bildung eines Produktgemisches aus Diazoniadiboratabicyclooctadienen und Azaazoniaboratacyclopentenen (vgl. Schema 7, Seite 30). Silylorganyle reagieren mit Halogenboranen $\mathrm{zu}$ Bororganylen und Halogensilanen. Dementsprechend reagiert disilyliertes tert-Butylmethylketazin II nach beendeter Butanabspaltung mit Trichlorboran unter Abspaltung von Trimethylchlorsilan zu folgenden Produkten ${ }^{[10]}$ :

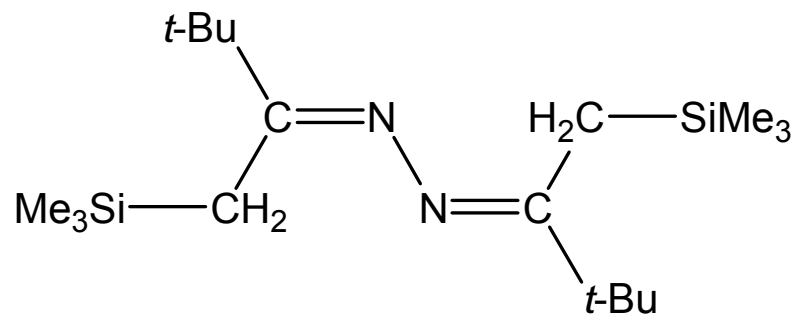

II

$$
\begin{array}{l|l}
+2 n-\mathrm{BuLi} & -2 \mathrm{n}-\mathrm{BuH} \\
+2 \mathrm{BCl}_{3} & -2 \mathrm{LiCl} \\
& -2 \mathrm{Me}_{3} \mathrm{SiCl}
\end{array}
$$<smiles>CS/C=C(\N1N=C(C(C)(C)C)CB1Cl)C(C)(C)C</smiles>

$40 \%$<smiles></smiles>

$60 \%$

Schema 13: Umsetzung der dilithiierten Verbindung II mit $\mathrm{BCl}_{3}$ 
Bringt man die dilithiierten Verbindungen I bzw. II mit Tetrachlorsilan zur Reaktion, entsteht das 5-tert-Butyl-3,3-dichlor-3,4-dihydro-2-(3,3-dimethylbut-1-en-2-yl)-2H1,2,3-diazasilol 19 bzw. das 5-tert-Butyl-3,3-dichlor-3,4-dihydro-2-(3,3-dimethyl-1(trimethylsilyl)but-1-en-2-yl)-4-(trimethylsilyl)-2H-1,2,3-diazasilol 20:<smiles>[R]CC([R15])=NN=C([R15])C[R]</smiles>

I bzw. ||

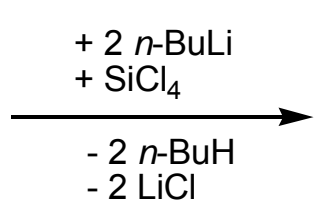

$-2 \mathrm{LiCl}$<smiles>[R]C=C([R15])N1N=C([R15])C([R])[Si]1(Cl)Cl</smiles>

19,20

\begin{tabular}{|c|c|c|}
\hline Verb. & 19 & 20 \\
\hline $\mathbf{R}$ & $\mathrm{H}$ & $\mathrm{SiMe}_{3}$ \\
\hline
\end{tabular}

Gleichung 30: Umsetzung der Verbindungen I und II mit SiCl 4

Beide Verbindungen sind hydrolyseempfindliche gelb-orange Flüssigkeiten. Das ${ }^{29}$ SiNMR-Signal der $\mathrm{SiCl}_{2}$-Gruppierung liegt bei 2,0 ppm (Verbindung 19) bzw. 1,6 ppm (Verbindung 20). Eine Abspaltung von Chlortrimethylsilan kann nicht beobachtet werden.

Die Verbindungen 19 und 20 müssten theoretisch geeignete Edukte für die Darstellung von stabilen Silylenen sein. Allerdings führten entsprechende Versuche in Anlehnung an WEST und DENK ${ }^{[69]}$ bisher zu keinem Erfolg. 


\subsubsection{Reaktionen mit Fluorsilanen}

\subsubsection{Umsetzungen mit Difluorsilanen}

Das Ketazin I wird mit zwei Moläquivalenten $n$-Butyllithium versetzt. Nach der abgeschlossenen Lithiierung wird die Reaktionslösung zu einem Moläquivalent Difluordiisopropylsilan bei $-50^{\circ} \mathrm{C}$ zugetropft. Das dilithiierte Ketazin I reagiert durch Eliminierung von zwei Molekülen Lithiumfluorid sowohl zum Diazasilacyclopenten 21 als auch zu der acyclischen disilylierten Verbindung 22:

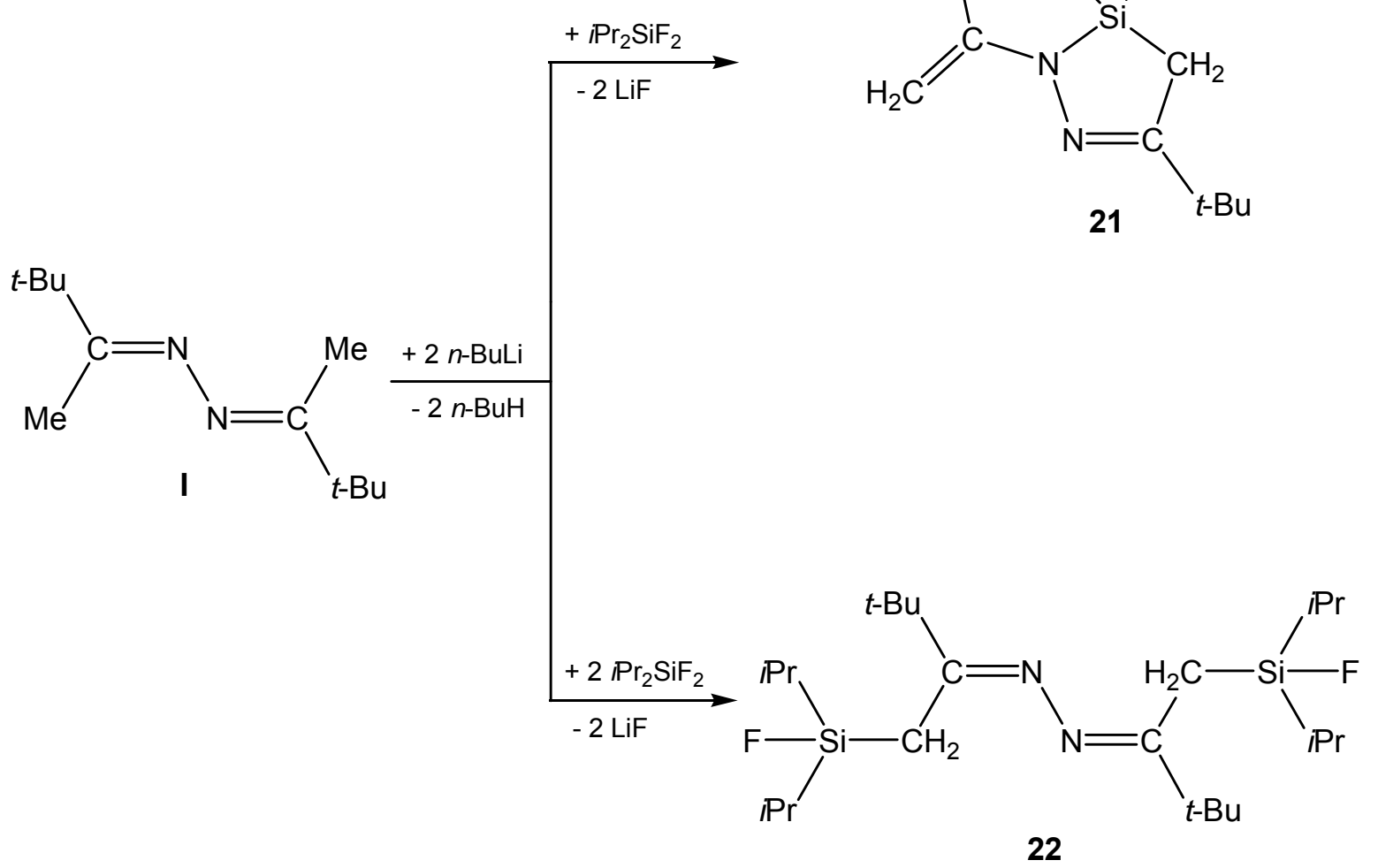

Gleichung 31: Reaktion des dilithiierten Ketazins I mit $\mathrm{PrSiF}_{2}$

Verbindung 21 lässt sich als gelbe Flüssigkeit mit einer Ausbeute von ca. $40 \%$ destillieren. Die Methylgruppen der Isopropylgruppe koppeln im ${ }^{1} \mathrm{H}-\mathrm{NMR}$-Spektrum zu dem benachbarten einzelnen Wasserstoffatom. Sie liefern ein Dublett mit einer ${ }^{3} \mathrm{~J}_{\mathrm{HH}}$-Kopplung von $7,4 \mathrm{~Hz}$. Die ungesättigte $=\mathrm{CH}_{2}$-Gruppierung zeigt ebenfalls ein Dublett mit einer ${ }^{2} \mathrm{~J}_{\mathrm{HH}}$-Kopplung von $0,8 \mathrm{~Hz}$. 
Verbindung 22 ist ein gelber Feststoff, der bei der Destillation aus der Vorlage erstarrt. Die Methylgruppen der Isopropylgruppierung koppeln ebenfalls mit ${ }^{3} \mathrm{~J}_{\mathrm{HF}}=7,4 \mathrm{~Hz}$. Die gesättigte $\mathrm{CH}_{2}$-Gruppe spaltet mit einer ${ }^{3} \mathrm{~J}_{\mathrm{HF}}=9,2 \mathrm{~Hz}$ zum Dublett auf. Das Dublett der $i \mathrm{Pr}_{2} \mathrm{SiF}-G r u p p e$ liegt im ${ }^{29} \mathrm{Si}-\mathrm{NMR}$ mit einer ${ }^{1} \mathrm{~J}_{\mathrm{SiF}}$ von $303 \mathrm{~Hz}$ bei 24,9 ppm.

22 lässt sich in $n$-Hexan umkristallisieren und röntgenstrukturanalytisch vermessen. In Abbildung 14 ist die Kristallstruktur von Verbindung 22 dargestellt:

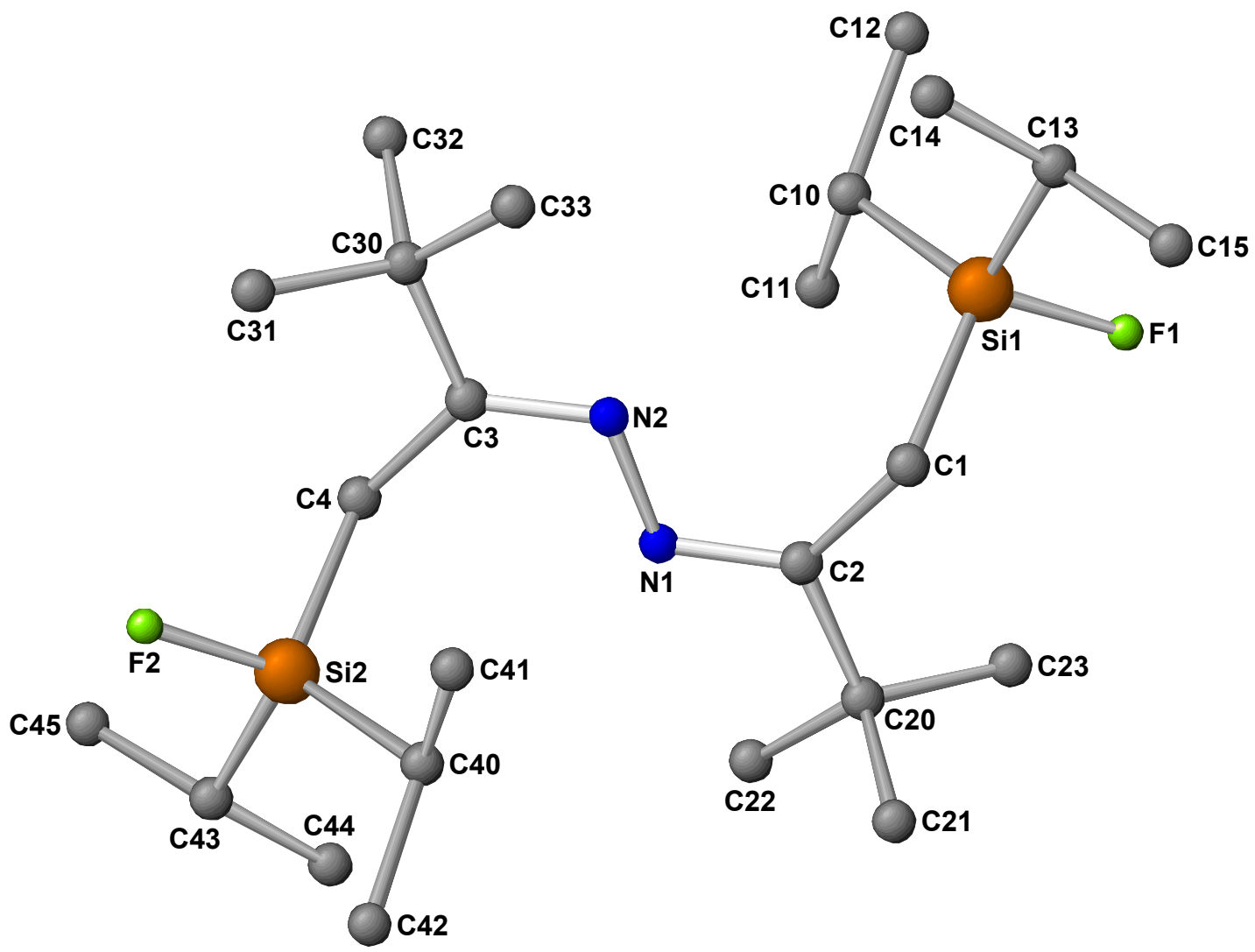

Abbildung 14: Kristallstruktur von Verbindung 22

\begin{tabular}{|l|r|}
\hline C1-C2 & $150,8 \mathrm{pm}$ \\
\hline C2-N1 & $128,5 \mathrm{pm}$ \\
\hline N1-N2 & $143,2 \mathrm{pm}$ \\
\hline N2-C3 & $127,8 \mathrm{pm}$ \\
\hline C3-C4 & $150,5 \mathrm{pm}$ \\
\hline C1-Si1 & $187,4 \mathrm{pm}$ \\
\hline C4-Si2 & $187,7 \mathrm{pm}$ \\
\hline
\end{tabular}

\begin{tabular}{|l|r|}
\hline Si1-F1 & $161,1 \mathrm{pm}$ \\
\hline Si2-F2 & $161,1 \mathrm{pm}$ \\
\hline C3-C30 & $152,8 \mathrm{pm}$ \\
\hline C2-C20 & $152,0 \mathrm{pm}$ \\
\hline Si1-C13 & $185,8 \mathrm{pm}$ \\
\hline Si1-C10 & $186,4 \mathrm{pm}$ \\
\hline
\end{tabular}

Tabelle 8: Ausgewählte Bindungslängen von Verbindung 22 
Verbindung 22 kristallisiert in der triklinen Raumgruppe P-1 mit vier Molekülen in der Elementarzelle. Die Atome C1-C2-N1-N2-C3-C4 bilden eine Ebene, aus der das Si1Atom um 47,4 pm und das Si2-Atom um 48,9 pm herausragen. Die CNN-Winkel betragen $113^{\circ}$ und die $\mathrm{C}=\mathrm{N}$-Doppelbindungen haben eine Länge von 127,8 pm bzw. 128,5 pm. Die Si-F-Bindungen liegen mit 161 pm im erwarteten Bereich. Am Si1Atom erwartet man aufgrund der vier gebundenen Nachbaratome und der sich daraus ergebenen tetraedrischen Geometrie die entsprechenden Tetraederwinkel. Der C1-Si1-C10-Winkel ist mit 115,3 geweitet, während der F1-Si1-C13-Winkel mit $105,0^{\circ}$ etwas kleiner ist.
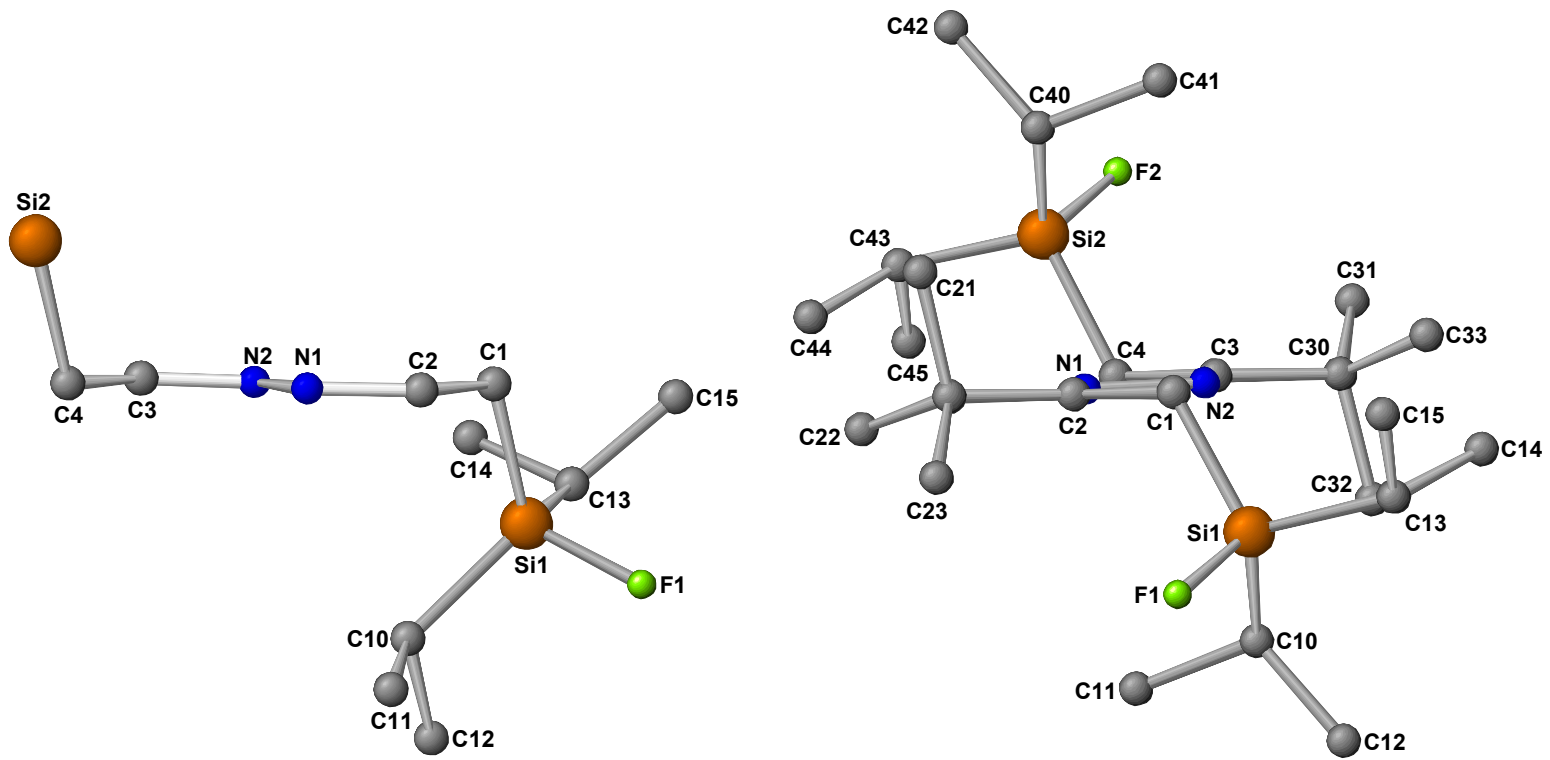

Abbildung 15: Seitenansichten der C1-C2-N1-N2-C3-C4-Ebene von Verbindung 22

\begin{tabular}{|l|l|}
\hline C1-C2-N1 & $124,2^{\circ}$ \\
\hline C2-N1-N2 & $113,4^{\circ}$ \\
\hline N1-N2-C3 & $113,1^{\circ}$ \\
\hline N2-C3-C4 & $124,8^{\circ}$ \\
\hline Si1-C1-C2 & $119,1^{\circ}$ \\
\hline F1-Si1-C1 & $108,2^{\circ}$ \\
\hline
\end{tabular}

\begin{tabular}{|l|l|}
\hline F1-Si1-C13 & $105,0^{\circ}$ \\
\hline C13-Si1-C10 & $113,6^{\circ}$ \\
\hline C13-Si1-C1 & $108,5^{\circ}$ \\
\hline C10-Si1-F1 & $105,5^{\circ}$ \\
\hline C1-Si1-C10 & $115,3^{\circ}$ \\
\hline
\end{tabular}

Tabelle 9: Ausgewählte Bindungswinkel von 22

Durch Umsetzung des dilithiierten Ketazins I mit Difluor-tert-butylphenylsilan erhält man das 3,5-Di-tert-butyl-3,4-dihydro-2-(3,3-dimethylbut-1-en-2-yl)-3-phenyl-2H1,2,3-diazasilol 23 in einer Ausbeute von rund $60 \%$ als orange, hoch viskose Flüssigkeit. 
<smiles>CC(C)(C)C(C)(C)C</smiles>

I<smiles>C=C(N1N=C(C(C)(C)C)C[Si]1(c1ccccc1)C(C)(C)C)C(C)(C)C</smiles>

23

Gleichung 32: Darstellung von Verbindung 23

Im ${ }^{1} \mathrm{H}-\mathrm{NMR}$-Spektrum beträgt die ${ }^{2} \mathrm{~J}_{\mathrm{HH}}-$ Kopplung der gesättigten Ring- $\mathrm{CH}_{2}-$ Gruppierung $19,0 \mathrm{~Hz}$. Die ungesättigte $=\mathrm{CH}_{2}$-Gruppierung liefert für jedes Wasserstoffatom ein Dublett mit einer ${ }^{2} \mathrm{~J}_{\mathrm{HH}}-$ Kopplung von $0,7 \mathrm{~Hz}$.

Wie bei Verbindung 15 sind Versuche, die gesättigte $\mathrm{CH}_{2}$-Gruppierung von Verbindung 23 mit Dihalogensilanen zu substituieren, bisher misslungen.

In einer weiteren Reaktion wird das dilithiierte Bis(tert-butyl-methyl)ketazin I bzw. die dilithiierte Verbindung 1 im Verhältnis $1: 1$ mit Difluordi-tert-butylsilan versetzt. Dabei entsteht das Diazasilepin 24 und das Diazasilacyclopenten 25:

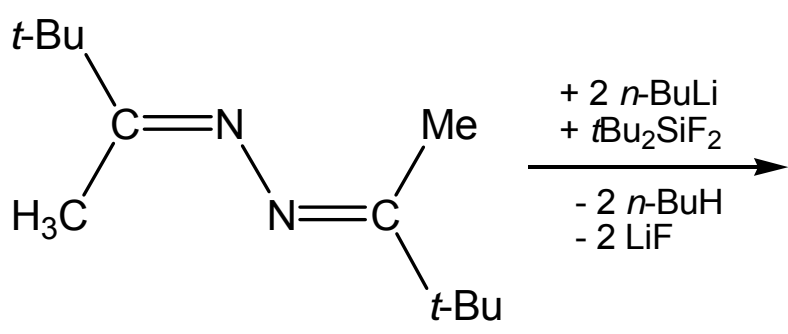

I<smiles>CC(C)(C)C1=CC(C(C)(C)C)=NN[Si](C(C)(C)C)(C(C)(C)C)C1</smiles>

24

Gleichung 33: Reaktion von I mit Difluordi-tert-butylsilan

Verbindung 24 ist eine gelbe Flüssigkeit, die im Hochvakuum unzersetzt destilliert werden kann. Das ${ }^{1} \mathrm{H}$-Spektrum zeigt für die NH-Gruppe ein Singulett bei 4,88 ppm und eine ungesättigte $=\mathrm{CH}$-Gruppierung bei 5,83 ppm. Im ${ }^{15} \mathrm{~N}-\mathrm{NMR}$-Spektrum findet man für die Aminogruppe ein Dublett mit einer ${ }^{1} \mathrm{~J}_{\mathrm{NH}}$ von $73,3 \mathrm{~Hz}$ bei $-276,1 \mathrm{ppm}$. 
Für die Bildung von Verbindung 24 wird in Anlehnung an Reaktionsweg $C$ (siehe Schema 12, Seite 36) folgender Mechanismus vorgeschlagen:<smiles>C/C(=N/N=C(\C)C(C)(C)C)C(C)(C)C</smiles><smiles>CC(C)(C)C1=CC(C(C)(C)C)=NN[Si](C(C)(C)C)(C(C)(C)C)C1</smiles>

24

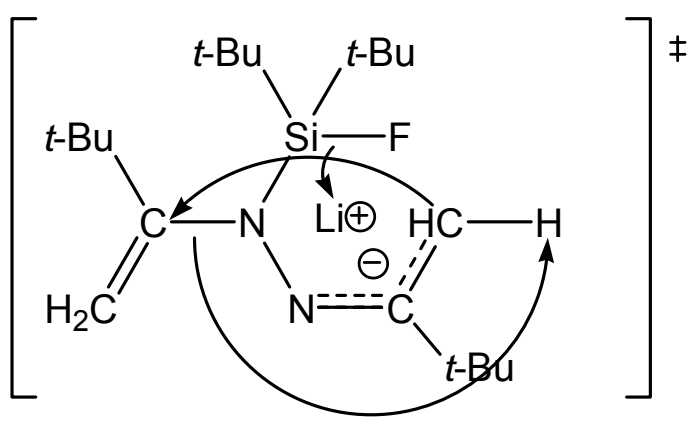

$\Delta \downarrow-\mathrm{LiF}$

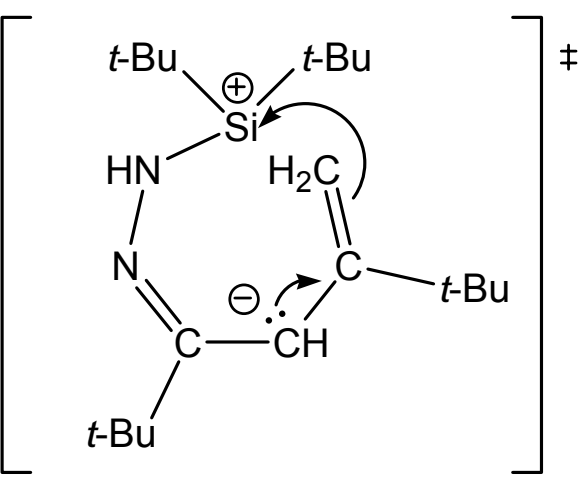

Schema 14: Möglicher Reaktionsmechanismus für die Darstellung von Verbindung 24

Die Fluordi-tert-butylsilyl-Gruppe bindet nach diesem Mechanismus am Stickstoffatom. Die C-N-Bindung wird durch drei sterisch anspruchsvolle tertButylgruppen in unmittelbarer Nachbarschaft geschwächt und bricht unter thermischer Belastung. Das entstehende Carbenium-lon lagert an der Methylengruppe an und es kommt zu einer Protonenwanderung zum Stickstoffatom. Das Molekül stabilisiert sich durch die Ausbildung eines konjugierten Systems mit zwei Doppelbindungen und dem freien Elektronenpaar der NH-Gruppierung zum siebengliedrigen Ring. 
<smiles>CC(C[SiH3])=NN=C(C)C(C)(C)C</smiles>

1<smiles>CC(C)(C)C(C)(C)C</smiles>

25

Gleichung 34: Reaktion von I mit Difluordi-tert-butylsilan

Verbindung 25 ist eine rötliche, hydrolyseunempfindliche Flüssigkeit, die in einer Ausbeute von $80 \%$ entsteht. Die ${ }^{29} \mathrm{Si}-\mathrm{NMR}-$ Verschiebung für die $t \mathrm{Bu}_{2} \mathrm{Si}-\mathrm{Gruppe}$ liegt bei 27,3 ppm und für die ungesättigte $=\mathrm{CSiMe}_{3}$-Gruppe bei $-11,9 \mathrm{ppm}$. 


\subsubsection{Synthese von fluorfunktionellen Diazasilacyclopentenen}

Bei der Reaktion des Dilithiumderivates von Verbindung I mit einem Äquivalent Trifluorsilan entstehen die Diazasilacyclopentene 26 - 28:<smiles>CC(C)(C)C(C)(C)C</smiles>

I

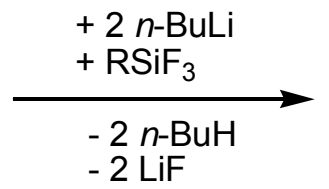

$-2 \mathrm{LiF}$<smiles></smiles>

$26,27,28$

\begin{tabular}{|c|c|c|c|}
\hline Verb. & $\mathbf{2 6}$ & $\mathbf{2 7}$ & $\mathbf{2 8}$ \\
\hline $\mathbf{R}$ & $t-\mathrm{Bu}$ & $\left(\mathrm{Me}_{3} \mathrm{Si}\right)_{2} \mathrm{~N}$ & $\left(\mathrm{Me}_{3} \mathrm{Si}\right)(t-\mathrm{Bu}) \mathrm{N}$ \\
\hline
\end{tabular}

Gleichung 35: Darstellung von fluorfunktionellen Diazasilacyclopentenen

Verbindung 26 erhält man als hellgelbe Flüssigkeit und die Verbindungen 27 und 28 als gelbe Feststoffe, die aus der Vorlage erstarren. Die Ausbeute beträgt jeweils etwa $60 \%$.

Verbindung 26 zeigt im ${ }^{1} \mathrm{H}$-NMR-Spektrum für die $\mathrm{CH}_{2}$-Gruppierungen ABX-Spektren und zwei ${ }^{3} \mathrm{~J}_{\mathrm{HF}}-$ Kopplungen von $17,1 \mathrm{~Hz}$ bzw. $6,0 \mathrm{~Hz}$. Die ${ }^{1} \mathrm{~J}_{\mathrm{SiF}}-$ Kopplung beträgt $339 \mathrm{~Hz}$.

Für die gesättigte $\mathrm{CH}_{2}$-Gruppe von Verbindung 27 findet man ebenfalls ein $\mathrm{ABX}$ Spektrum, während die ungesättigte $=\mathrm{CH}_{2}$-Gruppierung zum Dublett mit einer ${ }^{2} \mathrm{~J}_{\mathrm{HH}}=0,7 \mathrm{~Hz}$ bzw. Dublett von Dublett mit einer ${ }^{5} \mathrm{~J}_{\mathrm{HF}}=0,6 \mathrm{~Hz}$ aufspaltet. Die ${ }^{1} \mathrm{~J}_{\mathrm{SiF}^{-}}$ Kopplung von Verbindung 27 beträgt $282 \mathrm{~Hz}$.

Auch die gesättigte $\mathrm{CH}_{2}$-Gruppe der Verbindung 28 spaltet im ${ }^{1} \mathrm{H}$-NMR-Spektrum zum ABX-Spektrum auf. Die Protonen der ungesättigten $=\mathrm{CH}_{2}$-Gruppierung liefern jeweils ein Dublett mit einer ${ }^{2} J_{H H}=0,5 \mathrm{~Hz}$. Die ${ }^{1} J_{\mathrm{SiF}_{\mathrm{F}}}-$ Kopplung hat einen Wert von $276 \mathrm{~Hz}$.

Durch Umkristallisation der entstandenen Diazasilacyclopentene 27 und 28 in $n$ Hexan konnten röntgentaugliche Kristalle erhalten werden. Die Abbildungen 16 und 17 zeigen die Kristallstrukturen der Verbindungen $\mathbf{2 7}$ und 28. 


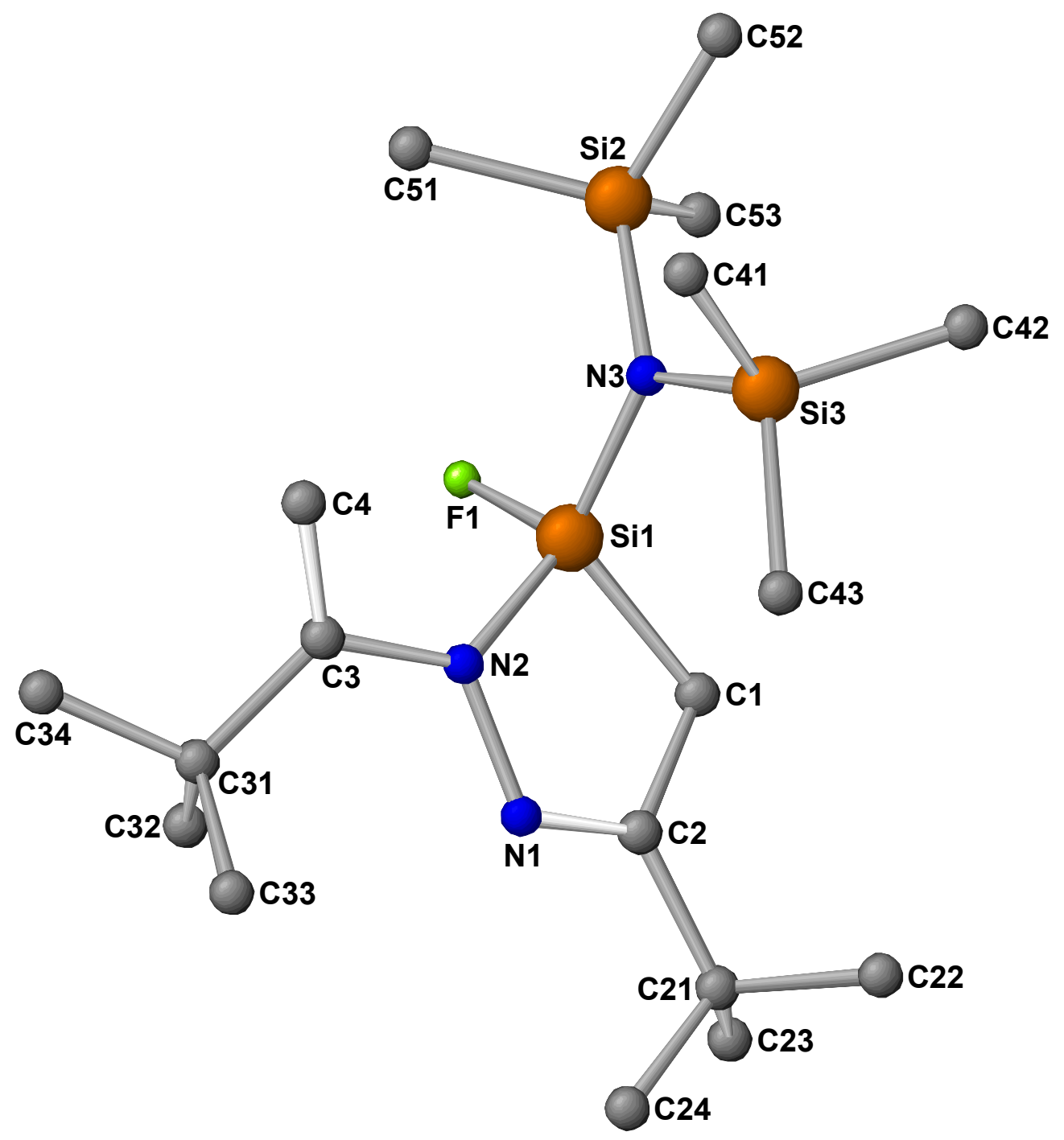

Abbildung 16: Kristallstruktur von Verbindung 27

\begin{tabular}{|l|c|c|}
\cline { 2 - 3 } \multicolumn{1}{c|}{} & $\mathbf{2 7}$ & $\mathbf{2 8}$ \\
\hline C1-C2 & $151,5 \mathrm{pm}$ & $151,2 \mathrm{pm}$ \\
\hline C2-N1 & $128,4 \mathrm{pm}$ & $128,5 \mathrm{pm}$ \\
\hline N1-N2 & $141,4 \mathrm{pm}$ & $141,7 \mathrm{pm}$ \\
\hline N2-C3 & $141,5 \mathrm{pm}$ & $141,5 \mathrm{pm}$ \\
\hline C3-C4 & $132,1 \mathrm{pm}$ & $132,6 \mathrm{pm}$ \\
\hline C1-Si1 & $184,9 \mathrm{pm}$ & $185,7 \mathrm{pm}$ \\
\hline N2-Si1 & $172,9 \mathrm{pm}$ & $173,6 \mathrm{pm}$ \\
\hline Si1-F1 & $159,5 \mathrm{pm}$ & $159,4 \mathrm{pm}$ \\
\hline Si1-N3 & $170,7 \mathrm{pm}$ & $170,9 \mathrm{pm}$ \\
\hline N3-Si2 & $176,8 \mathrm{pm}$ & $177,1 \mathrm{pm}$ \\
\hline N3-Si3 & $176,3 \mathrm{pm}$ & \\
\hline N3-C40 & & $153,3 \mathrm{pm}$ \\
\hline
\end{tabular}

Tabelle 10: Vergleich ausgewählter Bindungslängen von Verbindung 27 und 28 


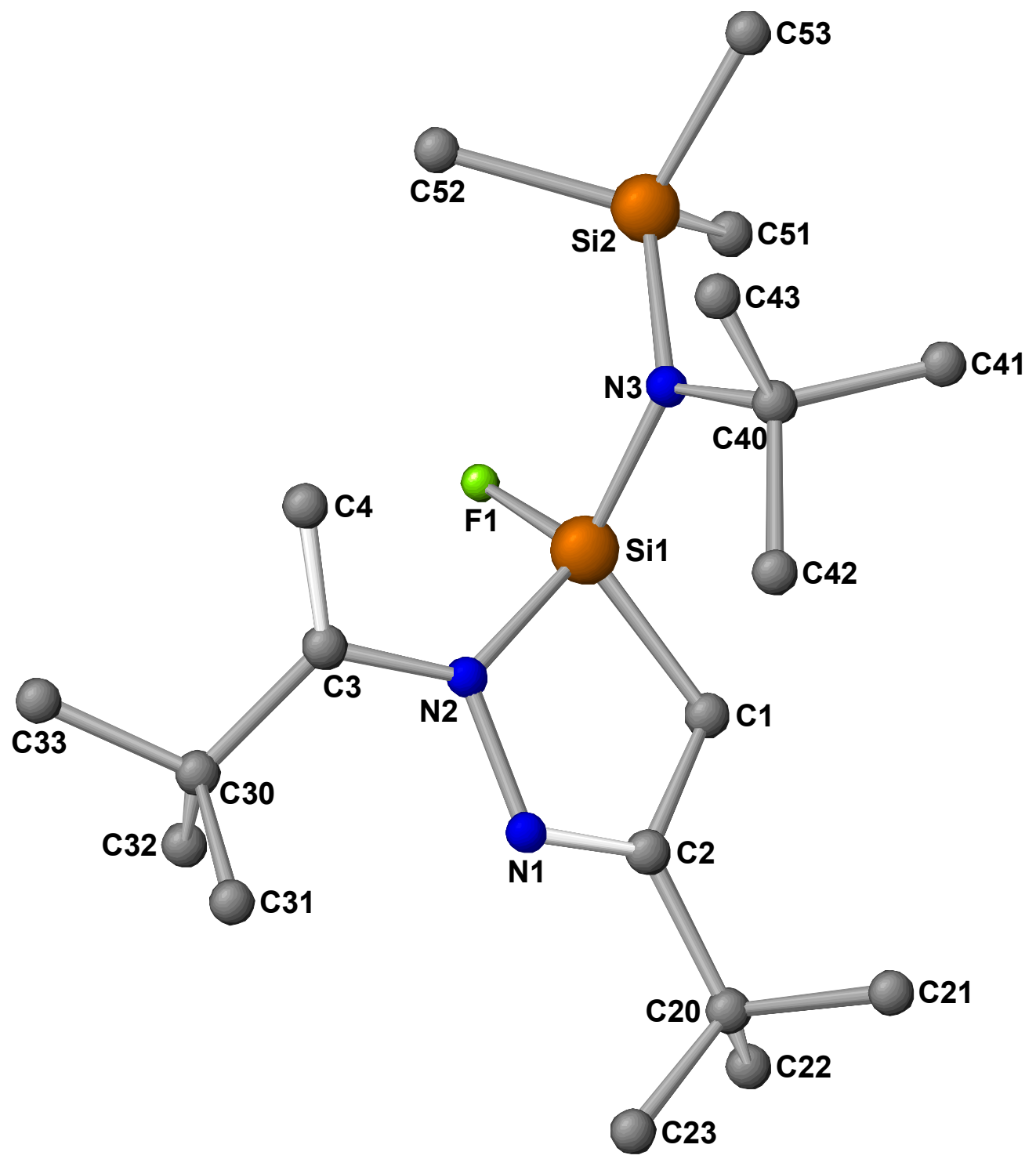

Abbildung 17: Kristallstruktur von Verbindung 28

Verbindung $\mathbf{2 7}$ und $\mathbf{2 8}$ bilden jeweils ein triklines Kristallsystem mit der Raumgruppe P-1 aus. Nach der Formel für ein gleichseitiges Vieleck $-180^{\circ} \cdot(n-2)=$ Innenwinkelsumme (mit $\mathrm{n}=$ Anzahl der Atome im Ring) - muss ein planarer fünfgliedriger Ring eine Innenwinkelsumme von $540^{\circ}$ aufweisen. Die Summe der Innenwinkel von Verbindung 27 beträgt $539,8^{\circ}$, von Verbindung $28539,0^{\circ}$. Beide C1-C2-N1-N2-Si1Ringe sind planar (vgl. Abbildung 18). Das C4-C3-N2-N1-Ketazinteilgerüst ist jeweils um rund $148^{\circ}$ tordiert. Die Winkelsumme an den N2-Atomen ergibt für Verbindung $27359,7^{\circ}$ und für Verbindung $28360,0^{\circ}$. Beide N2-Atome der Diazasilacyclopentene sind damit $\mathrm{sp}^{2}$-hybridisiert und trigonal planar. Die C2-N1Bindungen sind ca. 128 pm lang und die N1-N2-Einfachbindungen rund 141 pm. Die Si1-F1-Bindungen haben eine Länge von 159 pm und liegen damit im erwarteten 
Bereich. Die Si1-N3-Bindungen sind aufgrund des - I-Effektes der Fluoratome im Vergleich zu den N3-Si2-Bindungen um 6 pm kürzer.

\begin{tabular}{|l|c|c|}
\cline { 2 - 3 } \multicolumn{1}{c|}{} & $\mathbf{2 7}$ & $\mathbf{2 8}$ \\
\hline C1-C2-N1 & $118,7^{\circ}$ & $118,8^{\circ}$ \\
\hline C2-N1-N2 & $113,2^{\circ}$ & $113,3^{\circ}$ \\
\hline N1-N2-Si1 & $113,4^{\circ}$ & $112,9^{\circ}$ \\
\hline N2-Si1-C1 & $91,7^{\circ}$ & $91,5^{\circ}$ \\
\hline Si1-C1-C2 & $102,8^{\circ}$ & $102,5^{\circ}$ \\
\hline$\Sigma($ Innenwinkel) & $539,8^{\circ}$ & $539,0^{\circ}$ \\
\hline N1-N2-C3 & $117,7^{\circ}$ & $117,7^{\circ}$ \\
\hline N2-C3-C4 & $117,7^{\circ}$ & $117,7^{\circ}$ \\
\hline F1-Si1-N2 & $107,9^{\circ}$ & $106,9^{\circ}$ \\
\hline C1-Si1-F1 & $110,6^{\circ}$ & $110,0^{\circ}$ \\
\hline
\end{tabular}

Tabelle 11: Vergleich ausgewählter Bindungswinkel von Verbindung 27 und 28

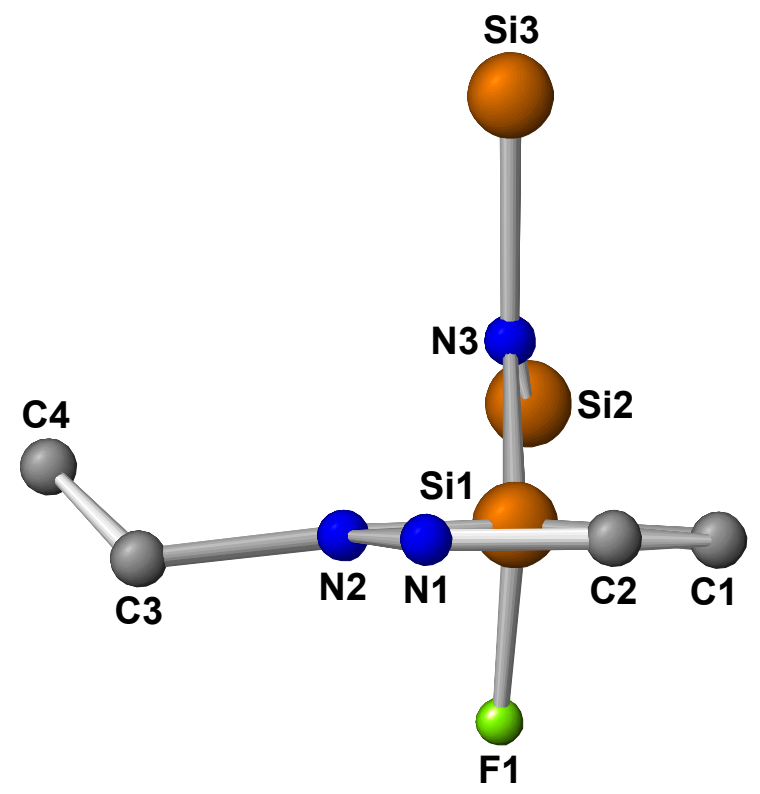

Verbindung 27

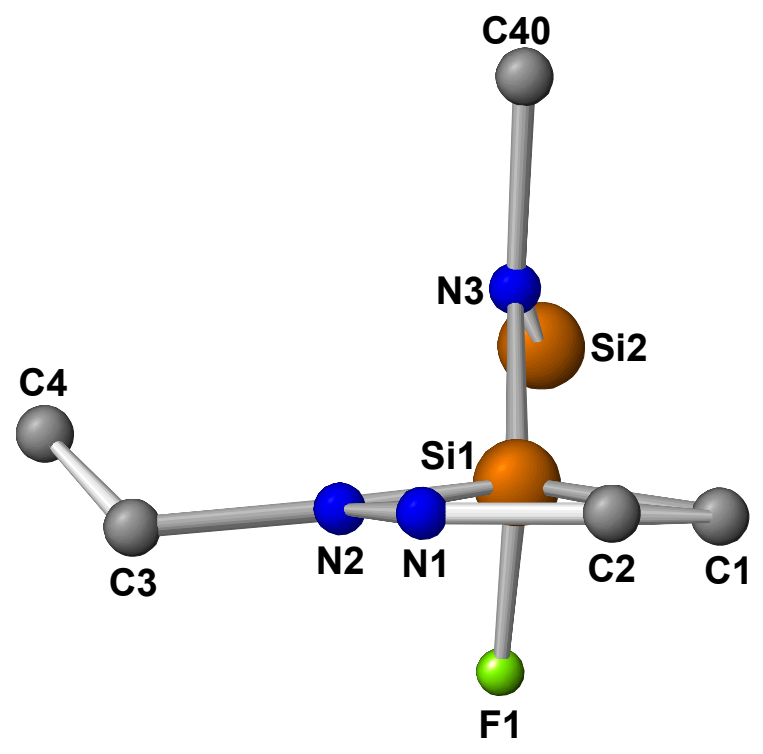

Verbindung 28

Abbildung 18: Seitenansichten der Ringebenen von Verbindung 27 und 28 
Wird das dilithiierte Bis(tert-butyl-(trimethylsilyl)methyl)ketazin II im Verhältnis $1: 1$ mit Trifluorsilanen umgesetzt, erhält man die Diazasilacyclopentene 29-32:

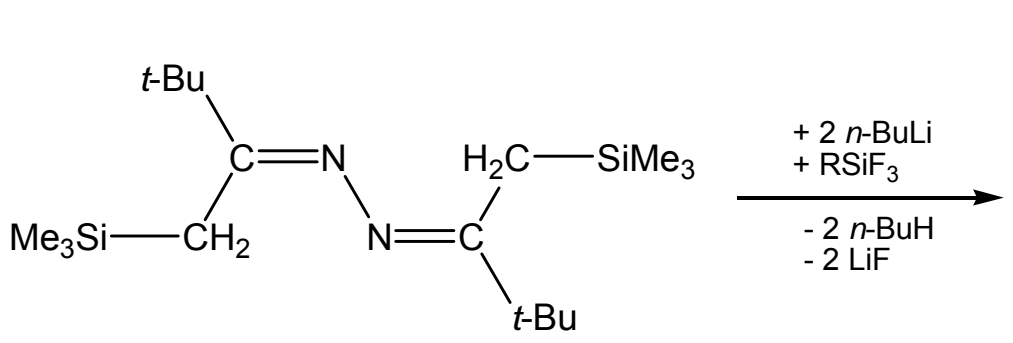

II

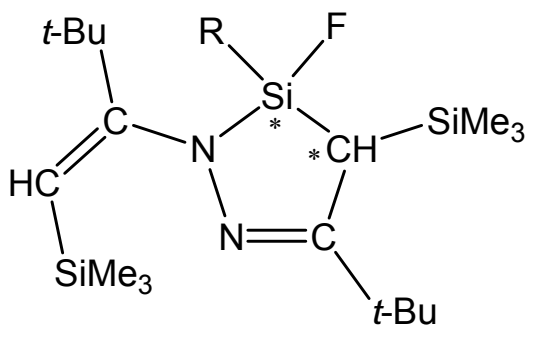

$29,30,31,32$

\begin{tabular}{|c|c|c|c|c|}
\hline Verb. & $\mathbf{2 9}$ & $\mathbf{3 0}$ & $\mathbf{3 1}$ & $\mathbf{3 2}$ \\
\hline $\mathbf{R}$ & $t-\mathrm{Bu}$ & $\mathrm{Ph}$ & $\left(\mathrm{Me}_{3} \mathrm{Si}\right)_{2} \mathrm{~N}$ & $\left(\mathrm{Me}_{3} \mathrm{Si}\right)(t-\mathrm{Bu}) \mathrm{N}$ \\
\hline
\end{tabular}

Gleichung 36: Reaktion des Bis(tert-butyl-(trimethylsilyl)methyl)ketazins II mit Trifluorsilanen

Die Verbindungen 29, 31 und 32 werden in Ausbeuten zwischen 60 und $70 \%$ erhalten und unterscheiden sich von den Verbindungen 26, 27 und 28 dadurch, dass $\mathrm{CHSiMe}_{3}$-Gruppierungen statt $\mathrm{CH}_{2}$-Gruppen vorliegen. Die Strukturen sollten deshalb im Festkörper annähernd identisch sein. Ein Beweis durch Röntgenstrukturanalyse steht noch aus. Wie Gleichung 36 verdeutlicht, besitzen die Verbindungen 29-32 zwei chirale Zentren und bilden zudem an der terminalen Butyliden-Doppelbindung (E/Z)Isomere. Alle vier Verbindungen fallen als Isomerengemisch und hoch viskose Flüssigkeit an. Einkristalle konnten aus den genannten Gründen bisher nicht gewonnen werden.

Aus den NMR-Daten wird deutlich: Es bilden sich im Allgemeinen zwei bis drei Isomere aus, die man unterscheiden und zuordnen kann. Um welchen Isomeren-Typ es sich jeweils handelt, ist anhand der NMR-Daten nicht klärbar.

Für das 3,5-Di-tert-butyl-3-fluor-3,4-dihydro-2-(3,3-dimethyl-1-(trimethylsilyl)but-1-en2-yl)-4-(trimethylsilyl)-2H-1,2,3-diazasilol 29 und das 5-tert-Butyl-3-fluor-3,4-dihydro2-(3,3-dimethyl-1-(trimethylsilyl)but-1-en-2-yl)-4-(trimethylsilyl)-3-phenyl-2H-1,2,3diazasilol 30 findet man im ${ }^{1} \mathrm{H}$-NMR-Spektrum ${ }^{7} \mathrm{~J}_{\mathrm{HF}}-$ Kopplungen im Bereich von $1,2 \mathrm{~Hz}$, deren Kopplungsstärken sich nur durch Raumeffekte begründen lassen. Die ${ }^{1} J_{\mathrm{SiF}}-$ Kopplungskonstanten nehmen von $329 \mathrm{~Hz}$ für Verbindung 29 bis $264 \mathrm{~Hz}$ für Verbindung 32 stetig ab. 


\subsubsection{Reaktionen mit Tetrafluorsilanen}

Bei Umsetzungen von Silylhydrazonen mit zwei Äquivalenten Butyllithium und anschließend bei $-30^{\circ} \mathrm{C}$ mit Tetrafluorsilan entstand die spirocyclische Verbindung 1,9-Bis(di-tert-butylmetylsilyl)-3,7-dimethyl-1,2,8,9-tetraaza-5-sila-spiro[4.4]nona-2,7dien ${ }^{[47]}$ :<smiles>CC[Si](C)(C)N(N=C(C)C)[Si](C)(C)C(C)(C)C</smiles>

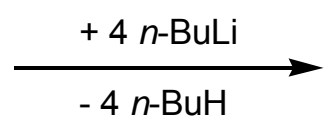

2<smiles>C/C(CCl)=N/N(Cl)[Si](C)(C(C)(C)C)C(C)(C)C</smiles><smiles>CC1=NN([Si](C)(C(C)(C)C)C(C)(C)C)[Si]2(C1)CC(C)=NN2[Si](C)(C(C)(C)C)C(C)(C)C</smiles>

Gleichung 37: Reaktion von dilithiierten Silyhydrazonen mit Tetrafluorsilan

Lässt man das Dilithiumderivat des Ketazins I bzw. des Bis(tert-butyl(trimethylsilyl)methyl)ketazins II mit einem Äquivalent Tetrafluorsilan reagieren, entstehen nicht die difluorfunktionellen Diazasilacyclopentene, sondern die spirocyclischen Verbindungen 33 und 34: 
<smiles>[R]CC(C[R])=NN=C(C[R])C(C)(C)C</smiles>

| bzw. ||

\begin{tabular}{l|l} 
& \\
$+4 n$-BuLi & $-4 n$-BuH \\
$+\mathrm{SiF}_{4}$ & $-4 \mathrm{LiF}$
\end{tabular}

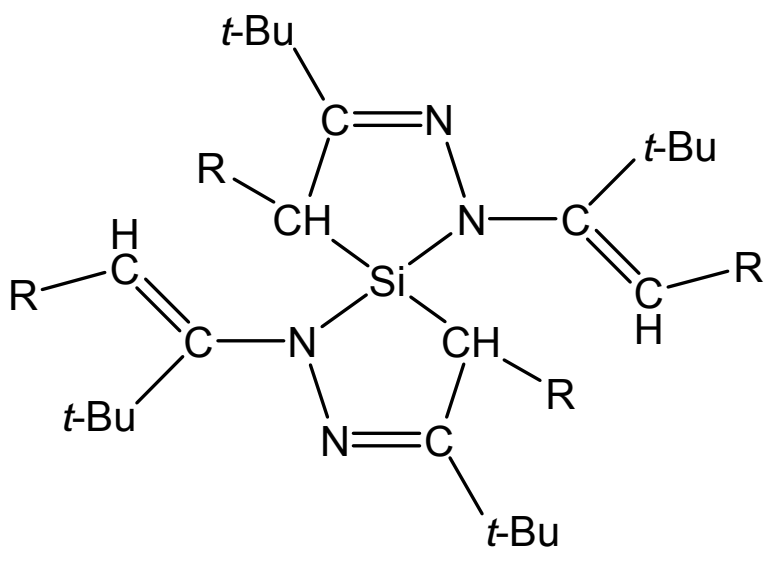

33,34

\begin{tabular}{|c|c|c|}
\hline Verb. & 33 & 34 \\
\hline $\mathbf{R}$ & $\mathrm{H}$ & $\mathrm{SiMe}_{3}$ \\
\hline
\end{tabular}

Gleichung 38: Reaktion von dilithiierten Ketazinen mit $\mathrm{SiF}_{4}$

Die Verbindungen 33 und 34 sind gelbe Feststoffe, die sich im Hochvakuum unzersetzt destillieren lassen.

Verbindung 33 zeigt im ${ }^{1} \mathrm{H}-\mathrm{NMR}$-Spektrum für die gesättigte $\mathrm{CH}_{2}$-Gruppe eine ${ }^{2} \mathrm{~J}_{\mathrm{HH}^{-}}$ Kopplung von $19,6 \mathrm{~Hz}$ und für die ungesättigte $=\mathrm{CH}_{2}$-Gruppierung beträgt die ${ }^{2} \mathrm{~J}_{\mathrm{HH}}=0,7 \mathrm{~Hz}$. Das ${ }^{29}$ Si-NMR-Signal hat eine Verschiebung von 9,8 ppm.

Verbindung 34 neigt wie die Diazasilacyclopentene 29-32 zur Isomerenbildung. Trotzdem konnten Einkristalle für die Röntgenstrukturanalyse durch Umkristallisation in $n$-Hexan gewonnen werden. 


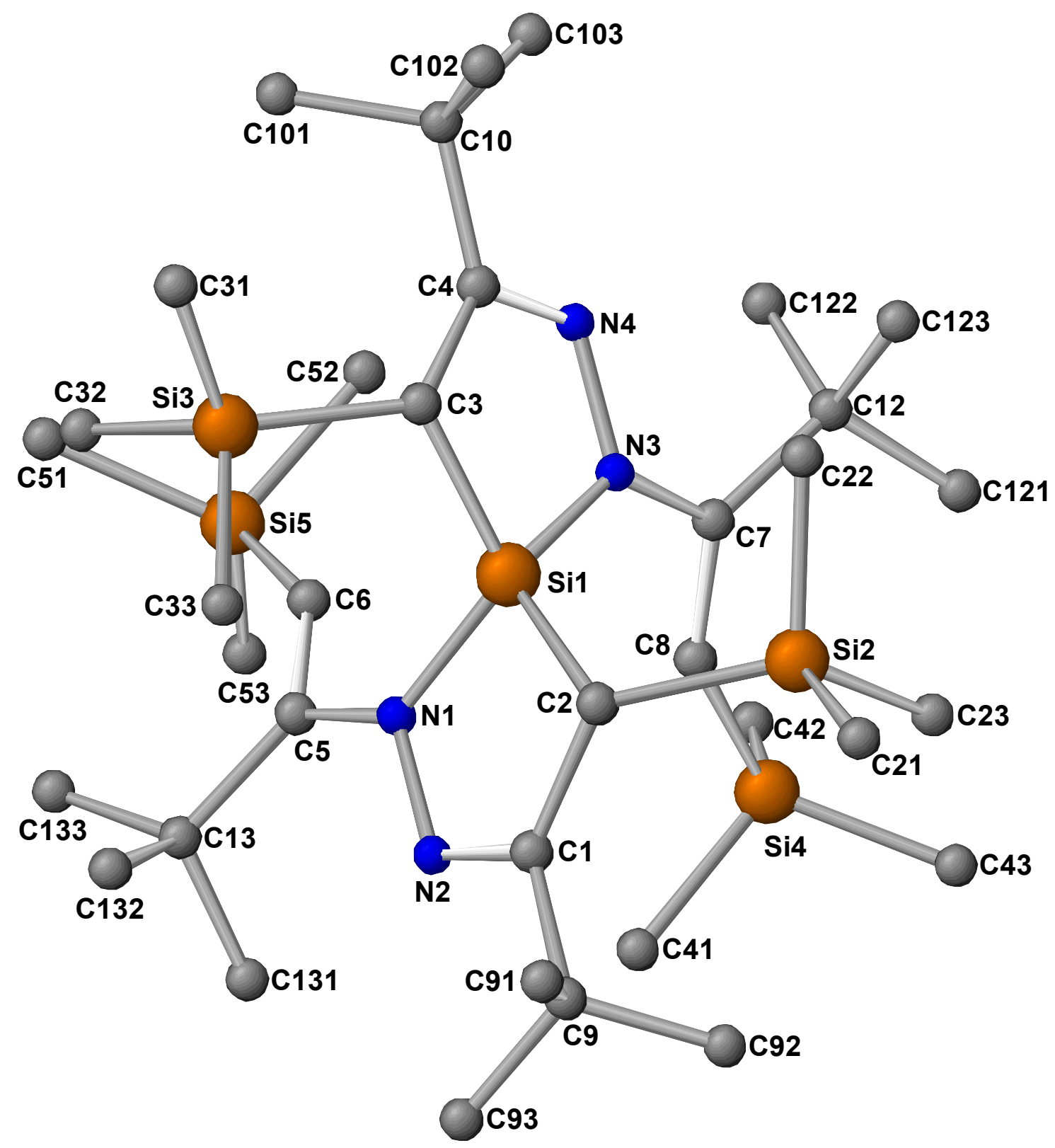

Abbildung 19: Kristallstruktur von Verbindung 34

\begin{tabular}{|l|r|}
\hline Si1-C2 & $187,4 \mathrm{pm}$ \\
\hline C2-C1 & $152,3 \mathrm{pm}$ \\
\hline C1-N2 & $128,2 \mathrm{pm}$ \\
\hline N2-N1 & $140,8 \mathrm{pm}$ \\
\hline N1-Si1 & $175,3 \mathrm{pm}$ \\
\hline N1-C5 & $142,3 \mathrm{pm}$ \\
\hline C5-C6 & $134,0 \mathrm{pm}$ \\
\hline C6-Si5 & $185,5 \mathrm{pm}$ \\
\hline C2-Si2 & $190,3 \mathrm{pm}$ \\
\hline C5-C13 & $153,9 \mathrm{pm}$ \\
\hline
\end{tabular}

\begin{tabular}{|l|l|}
\hline Si1-C3 & $187,9 \mathrm{pm}$ \\
\hline C3-C4 & $152,3 \mathrm{pm}$ \\
\hline C4-N4 & $128,3 \mathrm{pm}$ \\
\hline N4-N3 & $140,7 \mathrm{pm}$ \\
\hline N3-Si1 & $174,6 \mathrm{pm}$ \\
\hline N3-C7 & $143,4 \mathrm{pm}$ \\
\hline C7-C8 & $134,4 \mathrm{pm}$ \\
\hline C8-Si4 & $185,8 \mathrm{pm}$ \\
\hline C3-Si3 & $189,9 \mathrm{pm}$ \\
\hline C7-C12 & $153,5 \mathrm{pm}$ \\
\hline
\end{tabular}

Tabelle 12: Ausgewählte Bindungslängen von Verbindung 34 
Der Spirocyclus 34 kristallisiert in der monoklinen Raumgruppe P2(1)/n mit vier Molekülen in der Elementarzelle. Er besitzt eine kristallographisch zweizählige Achse, die durch das Si1-Atom läuft. Die C2-C1-N2-N1-Atome sind innerhalb 0,8 pm koplanar. Das Si1-Atom steht - 14,1 pm aus dieser Ebene heraus. Das C6-C5-N1N2-Teilgerüst des Ketazins ist um $-152,5^{\circ}$ tordiert. Die $\mathrm{C}=\mathrm{N}$-Doppelbindungen haben eine Länge von rund 128 pm und die N-N-Einfachbindungen sind etwa 141 pm lang. Auch die Si1-N-Bindungen liegen mit ca. $175 \mathrm{pm}$ im erwarteten Bereich. Die Atome N1 und N3 sind mit einer Winkelsumme von 359,3 ${ }^{\circ}$ bzw. 358,8 ${ }^{\circ} \mathrm{sp}^{2}-$ hybridisiert und trigonal planar. Der Spirocyclus 34 ist damit mit den bekannten, aus Hydrazonen dargestellten, Spirocyclen ${ }^{[47,68]}$ vergleichbar.

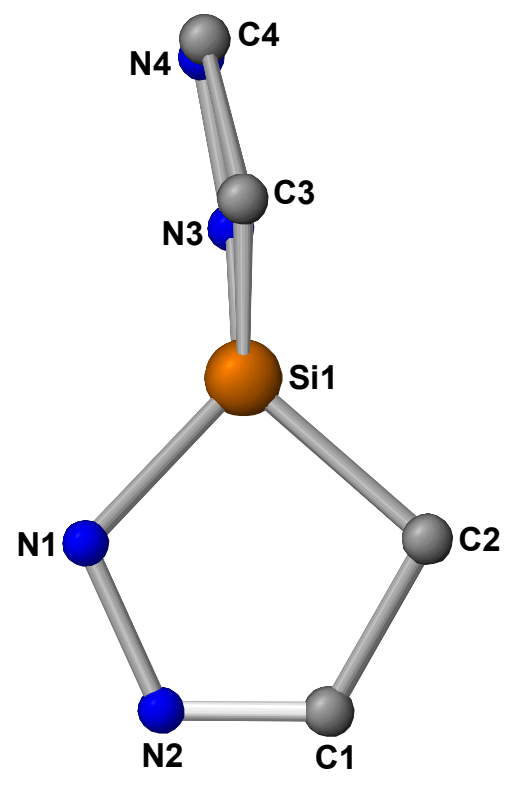

\begin{tabular}{|l|r|}
\hline N1-Si1-C2 & $92,1^{\circ}$ \\
\hline Si1-C2-C1 & $101,6^{\circ}$ \\
\hline C2-C1-N2 & $119,2^{\circ}$ \\
\hline C1-N2-N1 & $114,7^{\circ}$ \\
\hline N2-N1-Si1 & $112,0^{\circ}$ \\
\hline$\Sigma$ (Innenwinkel) & $539,6^{\circ}$ \\
\hline C5-N1-N2 & $117,3^{\circ}$ \\
\hline N2-N1-Si1 & $112,0^{\circ}$ \\
\hline Si1-N1-C5 & $130,0^{\circ}$ \\
\hline N1-C5-C6 & $116,3^{\circ}$ \\
\hline C5-C6-Si5 & $142,7^{\circ}$ \\
\hline Si1-C2-Si2 & $115,7^{\circ}$ \\
\hline
\end{tabular}

\begin{tabular}{|l|r|}
\hline N3-Si1-C3 & $91,8^{\circ}$ \\
\hline Si1-C3-C4 & $101,6^{\circ}$ \\
\hline C3-C4-N4 & $118,9^{\circ}$ \\
\hline C4-N4-N3 & $114,6^{\circ}$ \\
\hline N4-N3-Si1 & $112,2^{\circ}$ \\
\hline$\Sigma$ (Innenwinkel) & $539,1^{\circ}$ \\
\hline C7-N3-N4 & $116,2^{\circ}$ \\
\hline N4-N3-Si1 & $112,2^{\circ}$ \\
\hline Si1-N3-C7 & $130,4^{\circ}$ \\
\hline N3-C7-C8 & $116,3^{\circ}$ \\
\hline C7-C8-Si4 & $141,8^{\circ}$ \\
\hline Si1-C3-Si3 & $115,8^{\circ}$ \\
\hline
\end{tabular}

Tabelle 13: Ausgewählte Bindungswinkel von Verbindung 34 


\subsection{Weiterführende Reaktionen}

\subsubsection{Reaktion von Verbindung 12 mit Methanol}

Um die Struktur von Verbindung 12 aufzuklären, wird diese mit Methanol umgesetzt und reagiert quantitativ zum 2-(1-(Di-tert-butyl(methoxy)silyl)-3,3-dimethylbutan-2yliden)-1-(3,3-dimethylbutan-2-yliden)hydrazin 35:<smiles>CC(C)(C)C(C)(C)C</smiles>

12<smiles>CO[Si](C/C(=N\N=C(/C)C(C)(C)C)[13C](C)(C)C)(C(C)(C)C)C(C)(C)C</smiles>

35

Gleichung 39: Reaktion von Verbindung 12 mit Methanol

Verbindung 35 ist eine gelbe Flüssigkeit, die sich im Hochvakuum unzersetzt destillieren lässt. Das ${ }^{29}$ Si-NMR-Signal verschiebt sich durch die Addition des Methanols von 33,3 ppm (Verbindung 12) auf 9,5 ppm bei Verbindung 35.

Zur Kontrolle wird auch Verbindung 11 mit Methanol umgesetzt. Dabei erhält man ebenfalls als Produkt Verbindung 35:<smiles>C=C(N1N=C(C(C)(C)C)C[Si]1(C(C)(C)C)C(C)(C)C)C(C)(C)C</smiles>

11

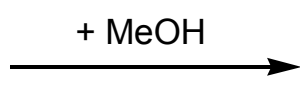

Gleichung 40: Umsetzung von Verbindung 11 mit Methanol 
Wie aus Gleichung 40 deutlich wird, spaltet Methanol die Si-N-Bindung. Eine Reaktion des Alkohols mit dem viergliedrigen Ring bzw. eine Addition an ein mögliches Silaethen (siehe Abbildung 8, Seite 27) würde demnach ebenfalls zur Entstehung von Verbindung 35 führen. Auf diesem Weg kann eine Entscheidung über die Struktur von Verbindung 12 deshalb nicht getroffen werden.

\subsubsection{Versuche zur Bildung einer Si=C-Doppelbindung}

Verbindung 29 weist ein acides Proton in Nachbarschaft zu einem Fluoratom in der gesättigten $\mathrm{CH}$-Gruppe des Ringes auf. Der Ring sollte sich an Position vier, wie für ähnliche Systeme beschrieben ${ }^{[42,43,45,48,50]}$, mit Lithiumalkylen metallieren lassen und zu einer Lithiumfluorid-Eliminierung eignen.

Bei der Reaktion von Verbindung 29 im Verhältnis $1: 1$ mit tert-Butyllithium konnte kein Produkt analysenrein isoliert werden. Umsetzungen mit Methyllithium, nButyllithium und Phenyllithium führen nicht $\mathrm{zu}$ einer intramolekularen Salzeliminierung, sondern zu den Substitutionsprodukten 36-38:

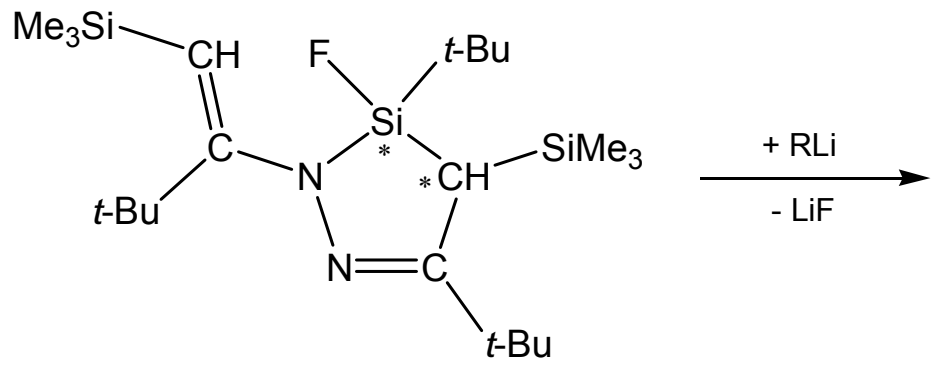

29

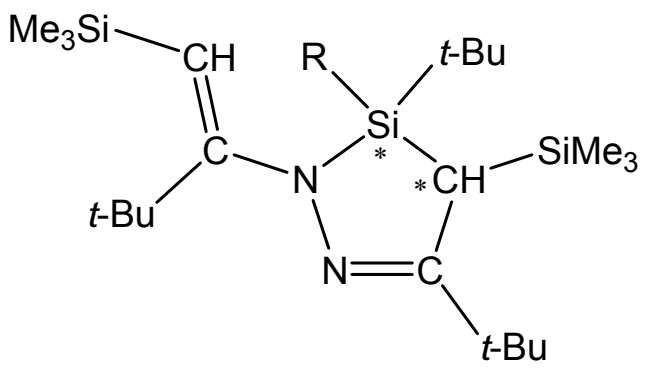

$36,37,38$

\begin{tabular}{|c|c|c|c|}
\hline Verb. & 36 & 37 & 38 \\
\hline $\mathbf{R}$ & $\mathrm{Me}$ & $n-\mathrm{Bu}$ & $\mathrm{Ph}$ \\
\hline
\end{tabular}

Gleichung 41: Reaktionen von Verbindung 29 mit Lithiumalkylen

Die Substitutionsprodukte $\mathbf{3 6}$ und $\mathbf{3 8}$ sind gelbe Feststoffe, Verbindung $\mathbf{3 7}$ ist eine gelborange Flüssigkeit. Die Produkte werden in einer Ausbeute von 70 bis $80 \%$ isoliert. 
Verbindung 29 wurde, wie bereits beschrieben, als Isomerengemisch im Verhältnis 2:1 erhalten, besitzt zwei chirale Zentren und kann zusätzlich (E/Z)-Isomere ausbilden. In den NMR-Spektren sind für die Substitutionsprodukte 36-38 keine Isomere zu erkennen. Dies wirft die Frage auf, wie aus einem Isomerengemisch selektiv ein Produkt synthetisiert werden kann. Dazu ist es sinnvoll, die möglichen Isomere des Eduktes 29 näher zu betrachten. In der folgenden Abbildung sind $(E)$ Isomere dargestellt. Die sterisch besonders anspruchsvollen Gruppen, die eine ungünstige räumliche Ausrichtung zueinander aufweisen, sind farbig hinterlegt.

\section{(E)-Isomere}

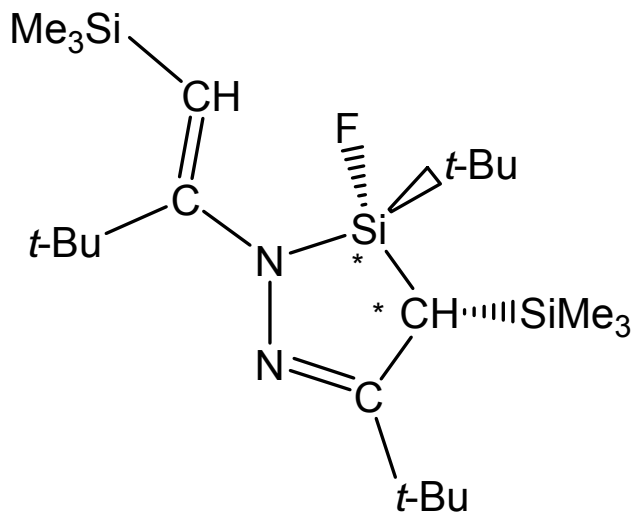

$(3 R, 4 S)-(E)$<smiles>C[SiH2]C=C(N1N=C(C(C)(C)C)[C@](F)(C(C)(C)C)[Si]1(F)C(C)(C)C)C(C)(C)C</smiles>

$(3 R, 4 R)-(E)$<smiles>CCCCC1=NN(/C(=C/[SiH3])C(C)(C)C)[Si](F)(F)[C@]1(CC(C)(C)C)C(C)(C)C</smiles>

$(3 S, 4 R)-(E)$<smiles>C[SiH2]C=C1N(/C(=C/[SiH3])C(C)(C)C)[Si](C)(C)(F)(F)C1(C)C</smiles>

$(3 S, 4 S)-(E)$

Abbildung 20: (E)-Isomere von Verbindung 29

In der (E)-Form gibt es vier verschiedene Konformationsmöglichkeiten: Ein $R, S / S, R-$ und ein R,R/S,S-Paar. Die Moleküle eines jeden Paares sind Spiegelbilder zueinander und damit optische Antipoden oder Enantiomere. Die einzelnen Isomere eines jeden Isomerenpaares verhalten sich $\mathrm{zu}$ dem jeweilig anderen Enantiomerenpaar nicht wie Bild und Spiegelbild, sie lassen sich nicht zur Deckung 
bringen. Es sind Diastereomere. Die R,S-Form ist also ein Enantiomer der $S, R$-Form und diastereomer zu den $R, R$ - und $S, S-F o r m e n$.

Im Gegensatz zu Enantiomeren, die sich NMR-spektroskopisch nicht unterscheiden lassen, besitzen Diastereomere unterschiedliche physikalische und chemische Eigenschaften und lassen sich im Kernspinresonanzspektrum nachweisen.

Die NMR-Spektren der Substitutionsprodukte 36-38 zeigen also nur, dass keine Diastereomere vorliegen, es kann sich aber durchaus um ein Enantiomerengemisch $(R, S / S, R$ bzw. $R, R / S, S)$ handeln. Wie aus Abbildung 20 ersichtlich wird, behindern sich die räumlich anspruchsvolle tert-Butyl- und Trimethysilylgruppe in der $R, R / S, S$ Konformation. Aus sterischen Gründen sollte deshalb das $R, S / S, R-$ Enantiomerenpaar bevorzugt gebildet werden.

Nachfolgend sind auch die (Z)-Isomere von Verbindung 29 aufgeführt. In der Abbildung sind wieder die Substituenten farbig hinterlegt, die sich gegenseitig sterisch behindern.

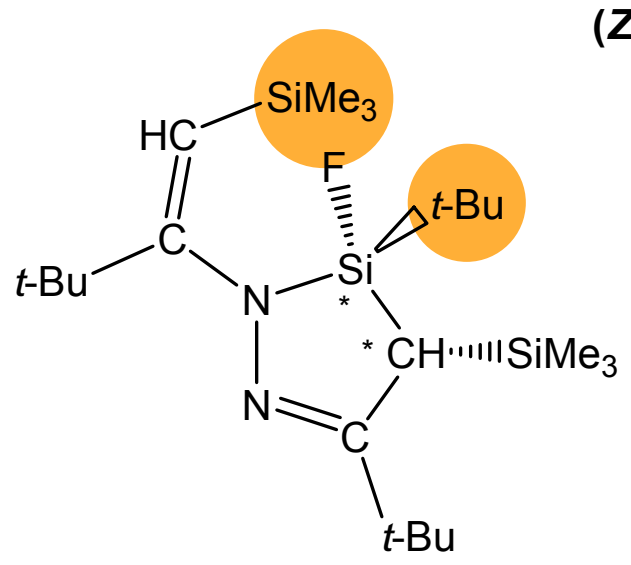

$(3 R, 4 S)-(Z)$

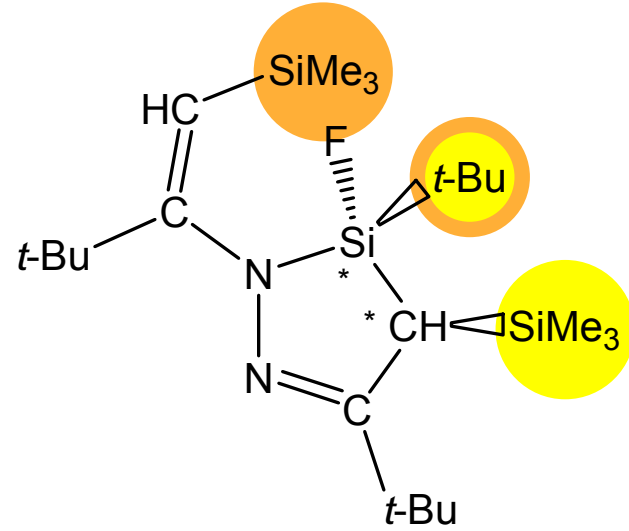

$(3 R, 4 R)-(Z)$

\section{(Z)-Isomere}
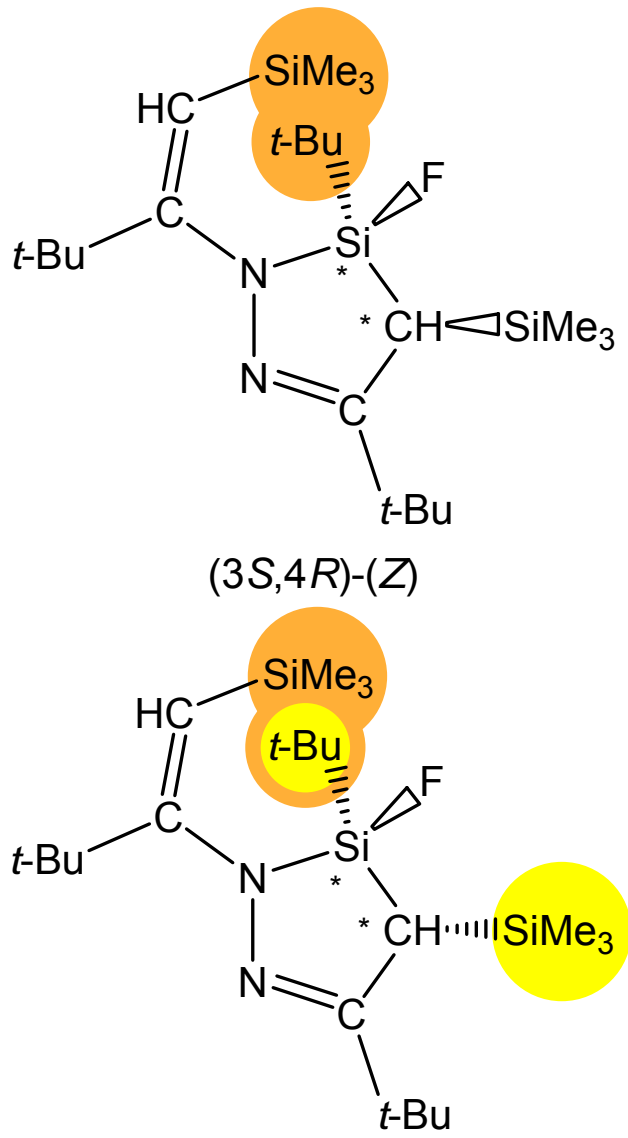

$(3 S, 4 S)-(Z)$

Abbildung 21: (Z)-Isomere von Verbindung 29 
Abbildung 21 macht deutlich, dass die Bildung aller (Z)-Isomere aufgrund der ungünstigen räumlichen Ausrichtung der Substituenten zueinander kinetisch unterbunden werden sollte.

Da Verbindung 29 im NMR-Spektrum ein Isomerengemisch im Verhältnis $2: 1$ bildet, ist $\mathrm{zu}$ vermuten, dass es sich dabei um die $(3 R, 4 S)-(E)-/(3 S, 4 R)-(E)-$ und $(3 R, 4 R)-(E)-/(3 S, 4 S)-(E)-$ Isomere handelt.

Die Röntgenstruktur des Substitutionsproduktes 36 zeigt das (3S,4S)-(E)-Isomer. Mit der Substitution ändert sich die Priorität an Position 3, was zu einer neuen Benennung führt. Die Kristallstruktur von Verbindung 36 entspricht aber dem $(3 R, 4 S)-$ (E)-Isomer des Eduktes 29. Andere Isomere werden im Festkörper nicht gefunden.

Eine Elementarzelle von Verbindung 38 beinhaltet die Enantiomere $(3 R, 4 S)-(E)$ und $(3 S, 4 R)-(E)$. Die Priorität an Position 3 ändert sich durch die Substitution im Vergleich zum Edukt 29 nicht. Die Isomerenbezeichnung ist deshalb identisch.

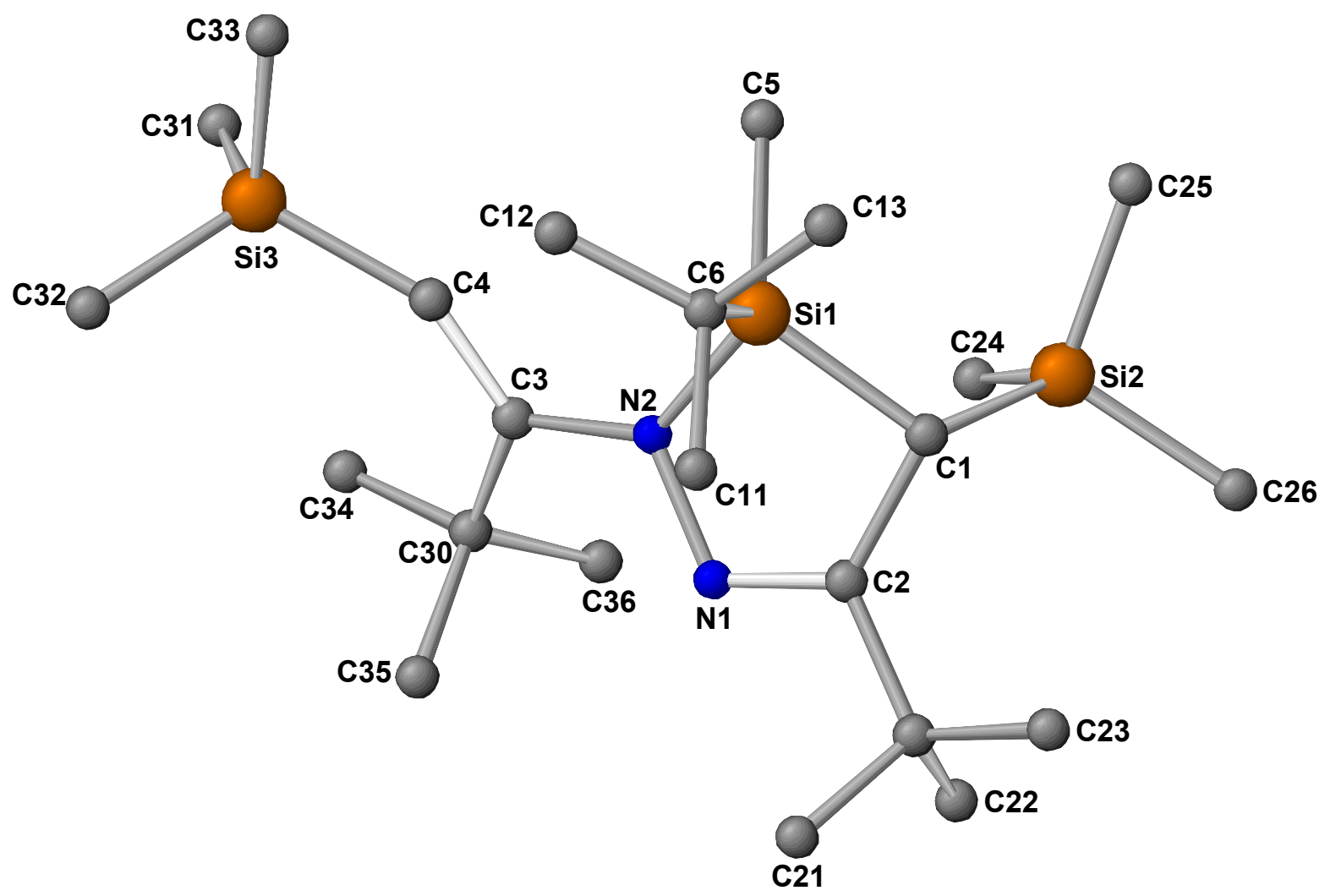

Abbildung 22: Kristallstruktur von Verbindung 36 


\begin{tabular}{|l|c|c|}
\cline { 2 - 3 } \multicolumn{1}{c|}{} & \multicolumn{1}{c|}{$\mathbf{3 6}$} & $\mathbf{3 8}$ \\
\hline C1-C2 & $152,8 \mathrm{pm}$ & $152,0 \mathrm{pm}$ \\
\hline C2-N1 & $128,0 \mathrm{pm}$ & $129,5 \mathrm{pm}$ \\
\hline N1-N2 & $142,4 \mathrm{pm}$ & $142,4 \mathrm{pm}$ \\
\hline N2-C3 & $140,5 \mathrm{pm}$ & $141,4 \mathrm{pm}$ \\
\hline C3-C4 & $135,7 \mathrm{pm}$ & $136,6 \mathrm{pm}$ \\
\hline C1-Si1 & $189,3 \mathrm{pm}$ & $190,6 \mathrm{pm}$ \\
\hline N2-Si1 & $176,7 \mathrm{pm}$ & $177,2 \mathrm{pm}$ \\
\hline Si1-C5 $(\mathrm{Me})$ & $186,2 \mathrm{pm}$ & \\
\hline Si1-C60 $(\mathrm{Ph})$ & & $187,9 \mathrm{pm}$ \\
\hline Si1-C6 $(t-B u)$ & $189,6 \mathrm{pm}$ & \\
\hline Si1-C50 $(t-\mathrm{Bu})$ & & $193,1 \mathrm{pm}$ \\
\hline C1-Si2 & $190,2 \mathrm{pm}$ & $192,9 \mathrm{pm}$ \\
\hline C4-Si3 & $185,7 \mathrm{pm}$ & $187,0 \mathrm{pm}$ \\
\hline
\end{tabular}

Tabelle 14: Vergleich ausgewählter Bindungslängen von Verbindung $\mathbf{3 6}$ und $\mathbf{3 8}$

Verbindung 36 kristallisiert in der triklinen Raumgruppe P-1 mit zwei Molekülen in der Elementarzelle. Verbindung 38 bildet ein monoklines Kristallsystem mit der Raumgruppe P2(1)/c und beinhaltet acht Moleküle in der Elementarzelle. Die N2-N1C2-C1-Atome von Verbindung 36 sind innerhalb 2,64 pm und von Verbindung 38 innerhalb 2,48 pm koplanar. Aus dieser Ebene ragt das Si1-Atom von 36 um 62,3 pm, das Si2- um 169,2 pm und das Si3-Atom um -55,4 pm heraus. Bei Verbindung 38 steht das Si1-Atom des (3S,4R)-(E)-Isomers um 59,9 pm, das Si2- um - 168,4 pm und das Si3-Atom 61,1 pm aus der aufgespannten Ebene hervor. Das C4-C3-N2-N1-Teilgerüst des Ketazins ist bei beiden Verbindungen um rund $150^{\circ}$ tordiert. Die N2-Atome sind mit einer Winkelsumme von 358,6 ${ }^{\circ}$ (36) und 357,9 ${ }^{\circ}$ (38) $\mathrm{sp}^{2}$-hybridisiert und trigonal planar. Alle Bindungslängen und Winkel liegen im erwarteten Bereich. 


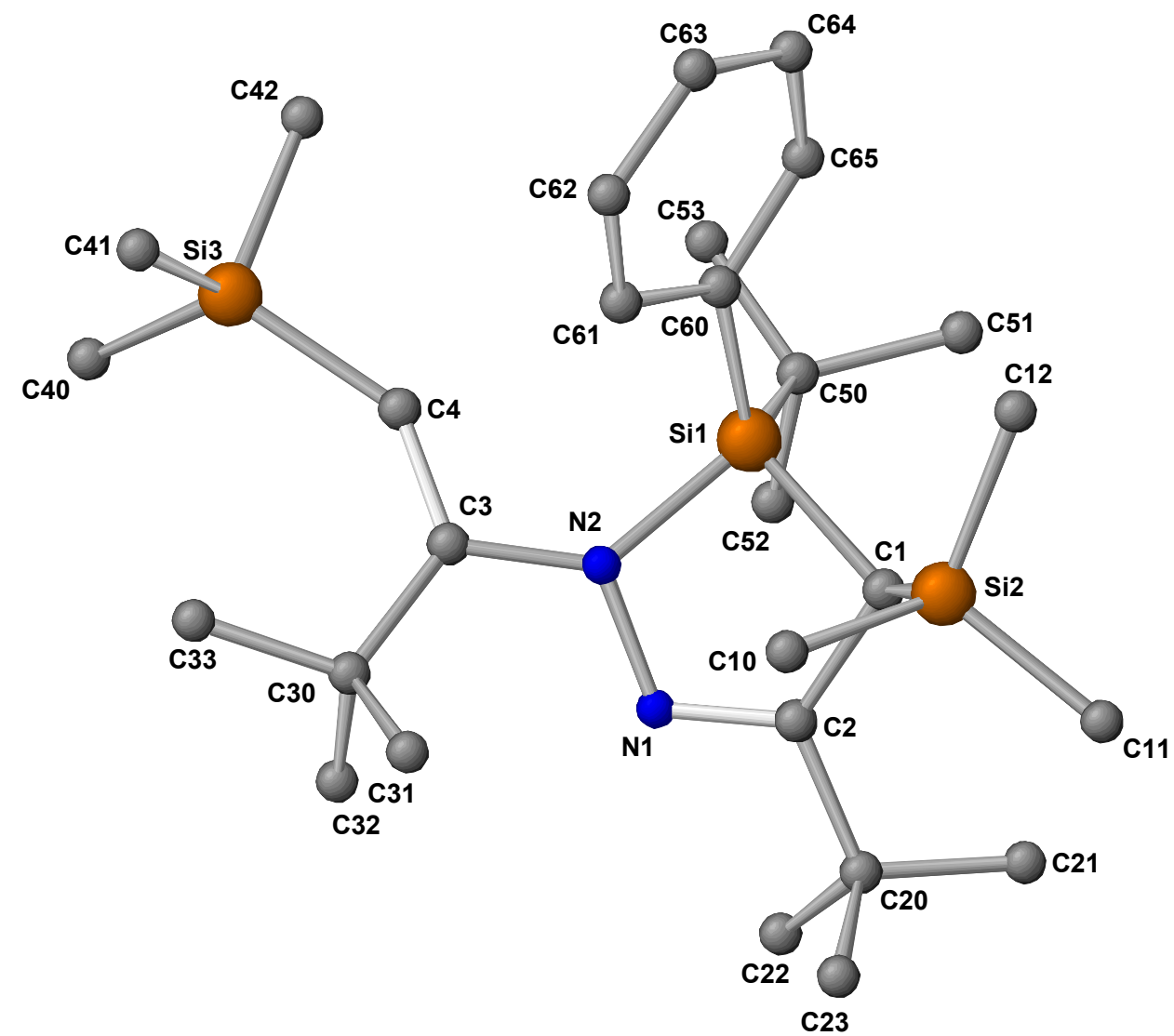

Abbildung 23: Kristallstruktur des $(3 S, 4 R)-(E)$-Isomers von Verbindung 38

\begin{tabular}{|l|c|c|}
\cline { 2 - 3 } \multicolumn{1}{c|}{} & $\mathbf{3 6}$ & $\mathbf{3 8}$ \\
\hline C1-C2-N1 & $119,8^{\circ}$ & $119,7^{\circ}$ \\
\hline C2-N1-N2 & $113,2^{\circ}$ & $113,6^{\circ}$ \\
\hline N1-N2-Si1 & $108,9^{\circ}$ & $109,0^{\circ}$ \\
\hline N2-Si1-C1 & $90,6^{\circ}$ & $90,8^{\circ}$ \\
\hline Si1-C1-C2 & $98,0^{\circ}$ & $98,4^{\circ}$ \\
\hline$\Sigma$ I Innenwinkel) & $530,5^{\circ}$ & $531,5^{\circ}$ \\
\hline N1-N2-C3 & $118,3^{\circ}$ & $117,4^{\circ}$ \\
\hline N2-C3-C4 & $117,0^{\circ}$ & $117,5^{\circ}$ \\
\hline N2-Si1-C5 & $112,7^{\circ}$ & \\
\hline N2-Si1-C60 & & $110,1^{\circ}$ \\
\hline N2-Si1-C6 & $115,0^{\circ}$ & \\
\hline N2-Si1-C50 & & $114,6^{\circ}$ \\
\hline
\end{tabular}

Tabelle 15: Ausgewählte Bindungswinkel von Verbindung $\mathbf{3 6}$ und $\mathbf{3 8}$ 


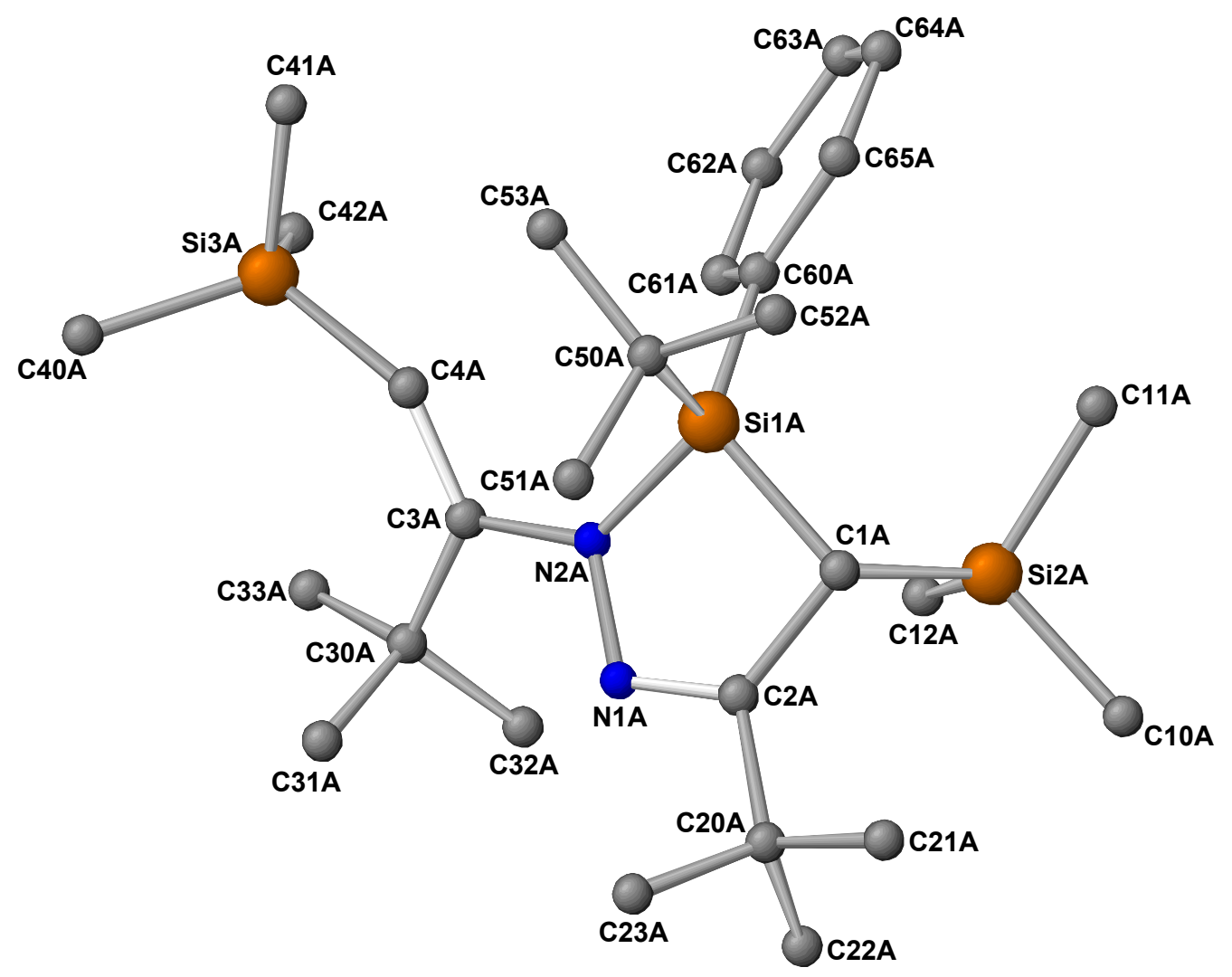

Abbildung 24: Kristallstruktur des (3R,4S)-(E)-Isomers von Verbindung 38

Die Abbildungen 25 und 26 verdeutlichen durch die Seitenansichten auf die Ringebenen der Verbindungen 36 und $\mathbf{3 8}$ die räumliche Ausrichtung der sterisch anspruchsvollen Substituenten.

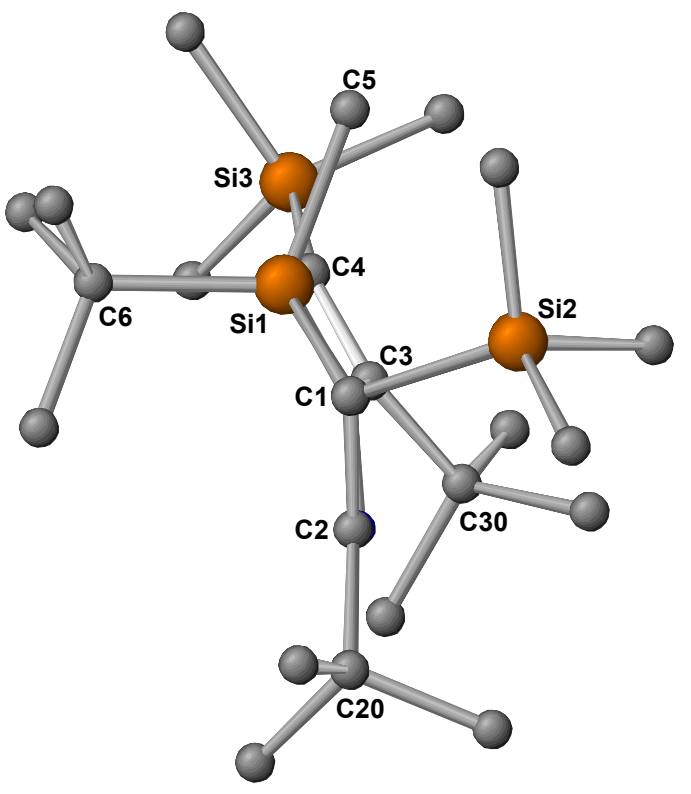

Blick auf die C1-C2-Bindung

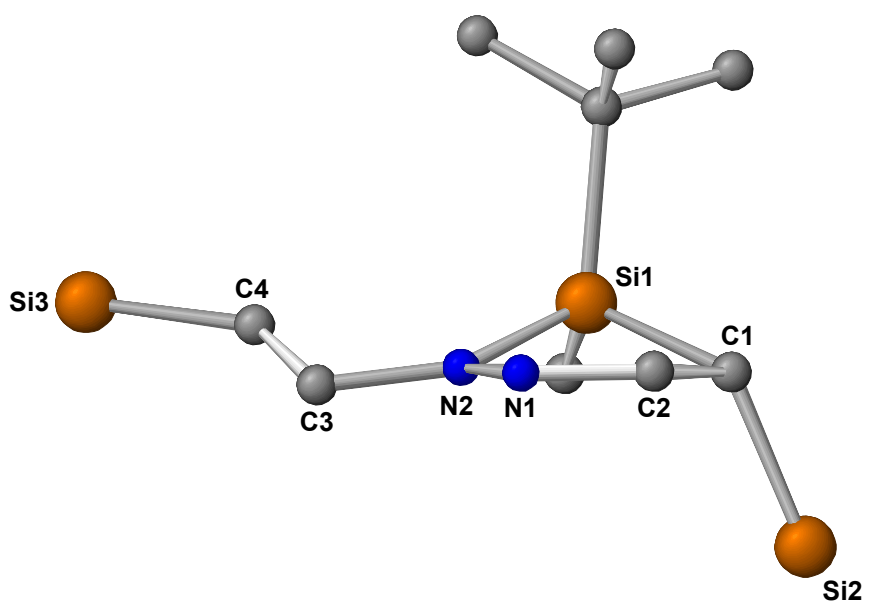

Blick auf die N1-C2-Bindung

Abbildung 25: Seitenansichten der Ringebene von Verbindung 36 
In Abbildung 26 sind die Enantiomere der Verbindung 38 einander gegenüber gestellt.

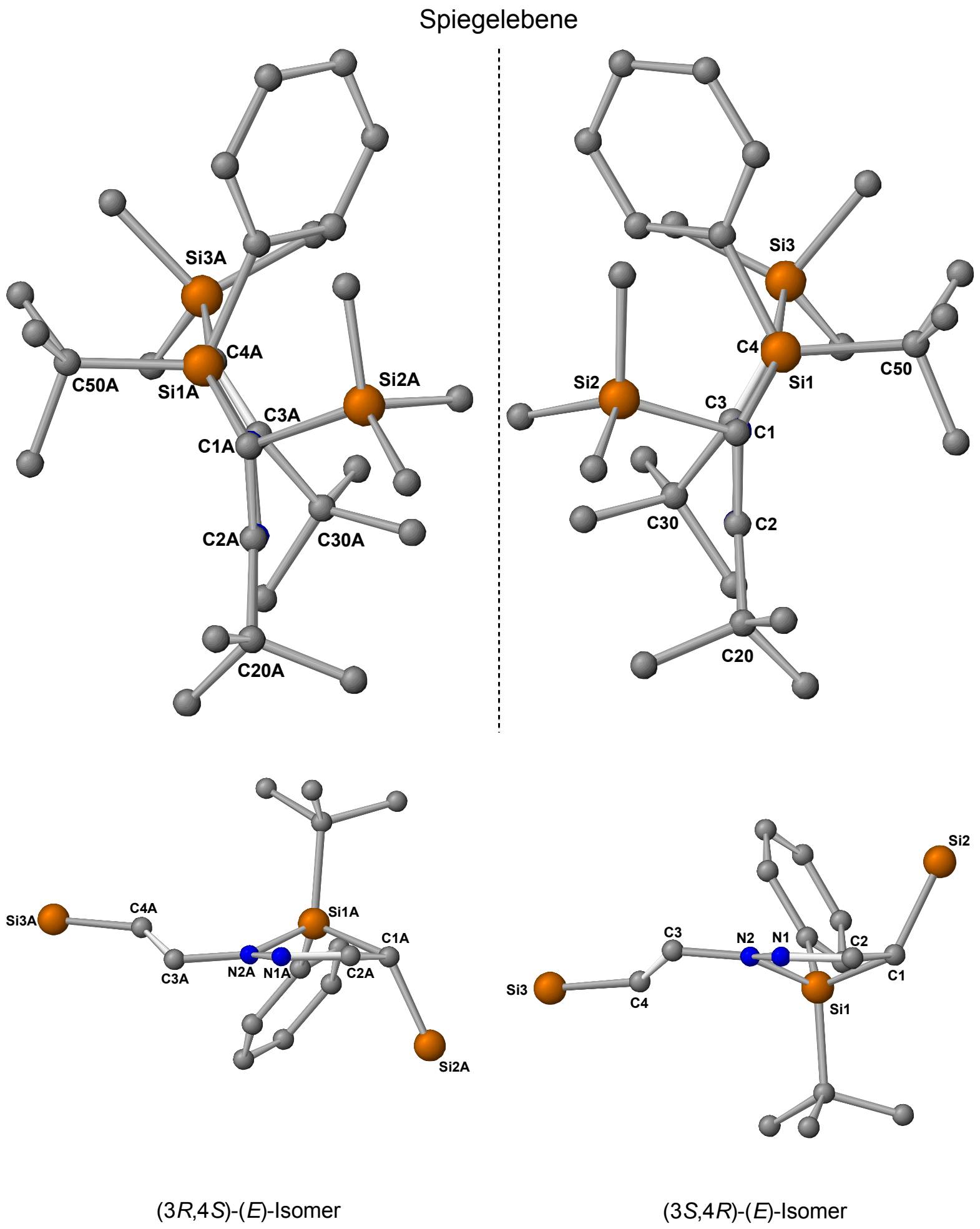

Abbildung 26: Seitenansichten der Ringebene von Verbindung 38 
Eine solche selektive Produktbildung aus einem diastereomeren und enantiomeren Eduktgemisch kann man durch einen $\mathrm{S}_{\mathrm{N}} 2-$ Mechanismus nicht erklären. Der $\mathrm{S}_{\mathrm{N}} 2-$ Mechanismus lässt sich in einen Vorderseiten- und einen Rückseitenangriff unterteilen. Im ersten Fall bleibt die Konfiguration des chiralen Zentrums erhalten, im zweiten Fall wird sie invertiert. Die $R, S-, S, R-, R, R$ - und $S, S-F o r m e n$ blieben daher beim Vorderseitenangriff bestehen - das Isomerengemisch müsste in den NMRSpektren im gleichen Verhältnis wieder $\mathrm{zu}$ finden sein. Im Falle des Rückseitenangriffs mit dem „Regenschirm“-Mechanismus würde jeweils das chirale Zentrum an Position 3 invertiert. Aus den R,S-, S,R-, R,R- und S,S-Isomeren entstehen die Formen $S, S, R, R, S, R$ und $R, S$. Wie beim Vorderseitenangriff bildet sich ein Isomerengemisch, in dem die Diastereomere in den Kernspinresonanzspektren unterscheidbar sein müssen und nur im umgekehrten Verhältnis vorliegen.

Eine Lösung bietet der $S_{N} 1$-Mechanismus unter Ausbildung eines intermediären Silylkations. Das angreifende Nucleophil nähert sich aus der sterisch weniger abgeschirmten Richtung und es wird selektiv das energetisch günstigere Produkt gebildet. Ein solcher Mechanismus ist für die Entstehung von Verbindung 38 im nachfolgenden Schema formuliert. Zur besseren Übersicht ist nur ein Isomer der jeweiligen Enantiomerenpaare ausgewählt worden. Stellvertretend für das $R, S / S, R-$ Paar ist das R,S-Isomer abgebildet, das $S, S$-Isomer steht für das $R, R / S, S$ Enantiomerenpaar. 
<smiles>CC(C)(C)C1=NN(/C(=C/[SiH3])C(C)(C)C)[Si](F)(C(C)(C)C)[C@H]1C(C)(C)C</smiles>

$29 \mathrm{~A}(3 R, 4 S)-(E)$

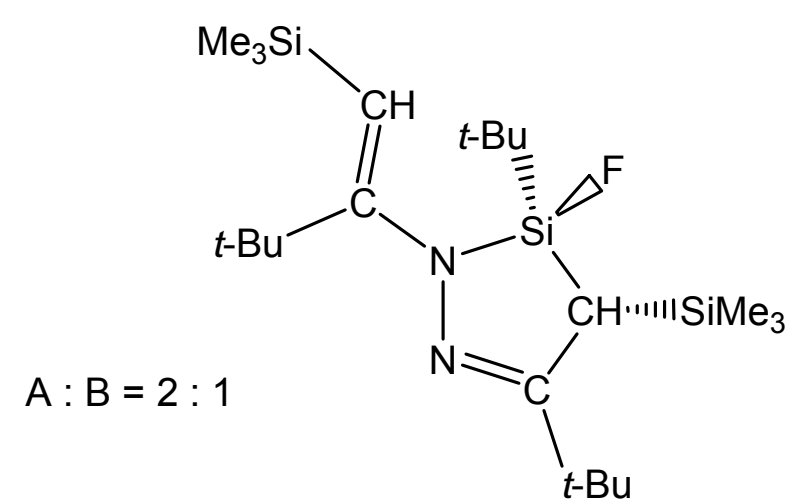

29 B $(3 S, 4 S)-(E)$

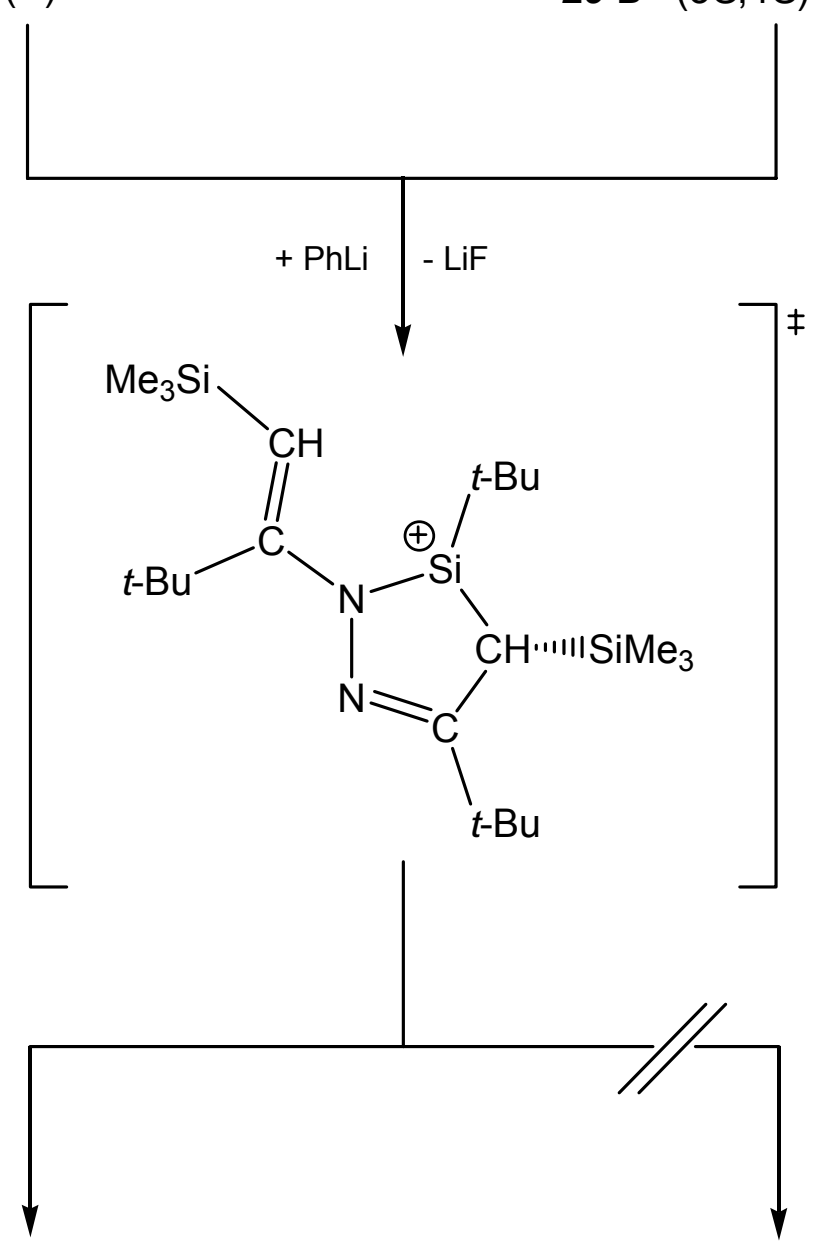

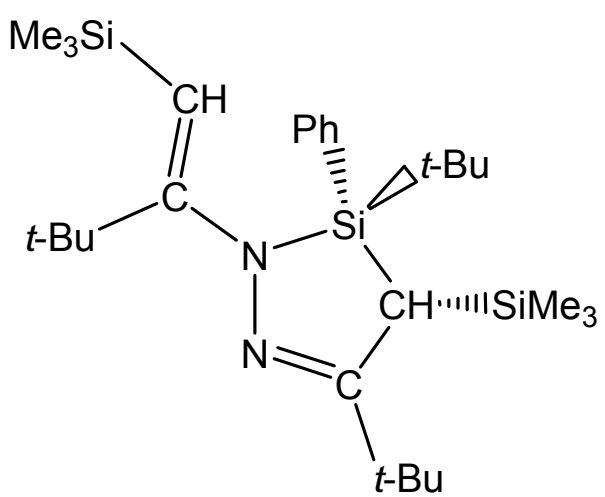

$38(3 R, 4 S)-(E)$

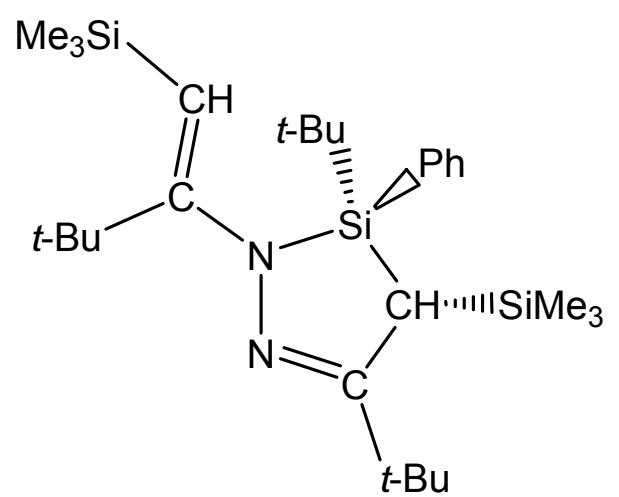

$(3 S, 4 S)-(E)$

Schema 15: Mögliche Produktbildung von Verbindung 38 über einen $S_{N} 1$-Mechanismus 
Da Silylkationen in der Siliciumchemie aber äußerst selten sind und erst kürzlich nachgewiesen werden konnten ${ }^{[70,71,72,73]}$, sind auch Mechanismen, die über einen fünf- oder sechsbindigen Übergangszustand des Siliciums laufen, denkbar.

WIBERG ${ }^{[60]}$ stellte sein erstes stabiles Silaethen durch thermische Salzeliminierung nach einem Fluor-Chloraustausch her:<smiles>CCC(C)(C)C(Cl)([AsH2])[Si](C)(C)F</smiles>

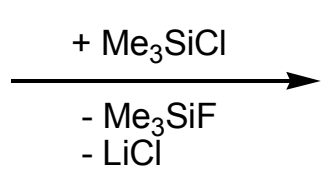<smiles>C[Si](C)C([AsH2])=[As](C)C</smiles>

Gleichung 42: Darstellung eines Silaethens

Analog dazu wird Verbindung 28 im Verhältnis 1:1 mit tert-Butyllithium und nach beendeter Butanabspaltung mit Chlortrimethylsilan umgesetzt. Dabei entsteht das Substitutionsprodukt $N$-(5-tert-Butyl-3-fluor-3,4-dihydro-2-(3,3-dimethylbut-1-en-2-yl)4-(trimethylsilyl)-2H-1,2,3-diazasilol-3-yl)-2-methyl-N-(trimethylsilyl)propan-2-amin 39:<smiles>C=C(N1N=C(C(C)(C)C)C[Si]1(F)N(C)C(C)(C)C)C(C)(C)C</smiles>

28<smiles>C=C(N1N=C(C(C)(C)C)[C@H](C)[Si]1(F)N([SiH3])C(C)(C)C)C(C)(C)C</smiles>

39

Gleichung 43: Darstellung von Verbindung 39

Verbindung 39 fällt bei der Destillation als orange, viskose Flüssigkeit an, die nach zwei Tagen in der Vorlage erstarrt.

Bei $500{ }^{\circ} \mathrm{C}$ im Hochvakuum über eine halbe Stunde zeigt Verbindung 39 keine Tendenz zur Fluortrimethylsilan-Abspaltung oder zur Zersetzung. 
Durch Umkristallisation in $n$-Hexan konnten von Verbindung 39 röntgentaugliche Einkristalle für die Strukturanalyse gewonnen werden. Die Kristallstruktur von $39 \mathrm{im}$ Festkörper ist in der folgenden Abbildung wiedergegeben:

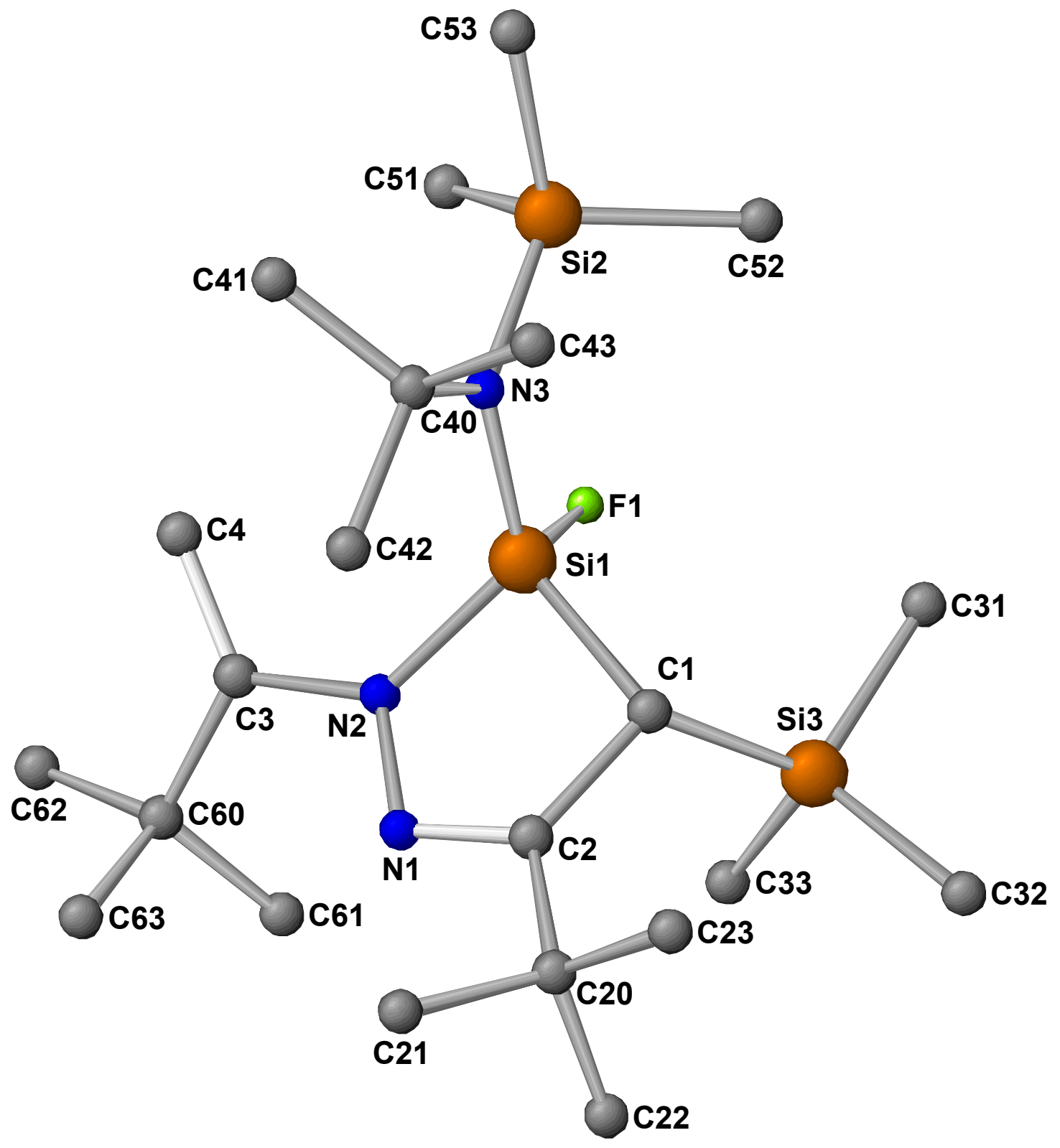

Abbildung 27: Kristallstruktur von Verbindung 39

In den Tabellen 16 und 17 sind zum Vergleich die Bindungslängen und -winkel der Ausgangsverbindung 28 noch einmal mit aufgeführt: 


\begin{tabular}{|l|c|c|}
\cline { 2 - 3 } \multicolumn{1}{c|}{} & $\mathbf{2 8}$ & $\mathbf{3 9}$ \\
\hline C1-C2 & $151,2 \mathrm{pm}$ & $152,2 \mathrm{pm}$ \\
\hline C2-N1 & $128,5 \mathrm{pm}$ & $128,6 \mathrm{pm}$ \\
\hline N1-N2 & $141,7 \mathrm{pm}$ & $141,3 \mathrm{pm}$ \\
\hline N2-C3 & $141,5 \mathrm{pm}$ & $142,8 \mathrm{pm}$ \\
\hline C3-C4 & $132,6 \mathrm{pm}$ & $132,5 \mathrm{pm}$ \\
\hline C1-Si1 & $185,7 \mathrm{pm}$ & $187,4 \mathrm{pm}$ \\
\hline N2-Si1 & $173,6 \mathrm{pm}$ & $173,4 \mathrm{pm}$ \\
\hline Si1-F1 & $159,4 \mathrm{pm}$ & $159,7 \mathrm{pm}$ \\
\hline Si1-N3 & $170,9 \mathrm{pm}$ & $171,4 \mathrm{pm}$ \\
\hline N3-Si2 & $177,1 \mathrm{pm}$ & $178,0 \mathrm{pm}$ \\
\hline N3-C40 & $153,3 \mathrm{pm}$ & $152,2 \mathrm{pm}$ \\
\hline C1-Si3 & & $192,3 \mathrm{pm}$ \\
\hline
\end{tabular}

Tabelle 16: Vergleich ausgewählter Bindungslängen des Eduktes 28 mit dem Reaktionsprodukt 39

Verbindung 39 bildet ein monoklines Kristallsystem mit der Raumgruppe P2(1)/c mit vier Molekülen in der Elementarzelle. Die endocyclische C1-Si1-Bindung von Verbindung 39 ist um rund $5 \mathrm{pm}$ kürzer als die exocyclische C1-Si3-Bindung. Die Substitution eines Protons durch eine Trimethylsilylgruppe verlängert die C1-Si1Bindung im Vergleich zum Edukt 28 um 1,7 pm. Alle anderen Bindungslängen und -winkel liegen im typischen Bereich. Das C4-C3-N2-N1-Teilgerüst des Ketazins ist um $145^{\circ}$ tordiert und die N2-N1-C2-C1-Atome sind innerhalb 3,32 pm koplanar. Das Si1-Atom steht aus dieser Ebene - 42,2 pm und das Si3-Atom 170,4 pm heraus. Das Fluoratom weicht um 67,8 pm von der Ebene ab.

\begin{tabular}{|l|c|c|}
\cline { 2 - 3 } \multicolumn{1}{c|}{} & $\mathbf{2 8}$ & $\mathbf{3 9}$ \\
\hline C1-C2-N1 & $118,8^{\circ}$ & $118,8^{\circ}$ \\
\hline C2-N1-N2 & $113,3^{\circ}$ & $113,4^{\circ}$ \\
\hline N1-N2-Si1 & $112,9^{\circ}$ & $111,9^{\circ}$ \\
\hline N2-Si1-C1 & $91,5^{\circ}$ & $91,3^{\circ}$ \\
\hline Si1-C1-C2 & $102,5^{\circ}$ & $100,4^{\circ}$ \\
\hline$\Sigma($ Innenwinkel) & $539,0^{\circ}$ & $535,8^{\circ}$ \\
\hline N1-N2-C3 & $117,7^{\circ}$ & $117,3^{\circ}$ \\
\hline N2-C3-C4 & $117,7^{\circ}$ & $117,8^{\circ}$ \\
\hline F1-Si1-N2 & $106,9^{\circ}$ & $106,2^{\circ}$ \\
\hline C1-Si1-F1 & $110,0^{\circ}$ & $111,8^{\circ}$ \\
\hline
\end{tabular}

Tabelle 17: Vergleich ausgewählter Bindungswinkel des Eduktes 28 mit dem Reaktionsprodukt 39 
Abbildung 28 ermöglicht durch Seitenansichten der Ringebene von Verbindung 39 einen optischen Vergleich mit den Verbindungen $\mathbf{3 6}$ und $\mathbf{3 8 .}$

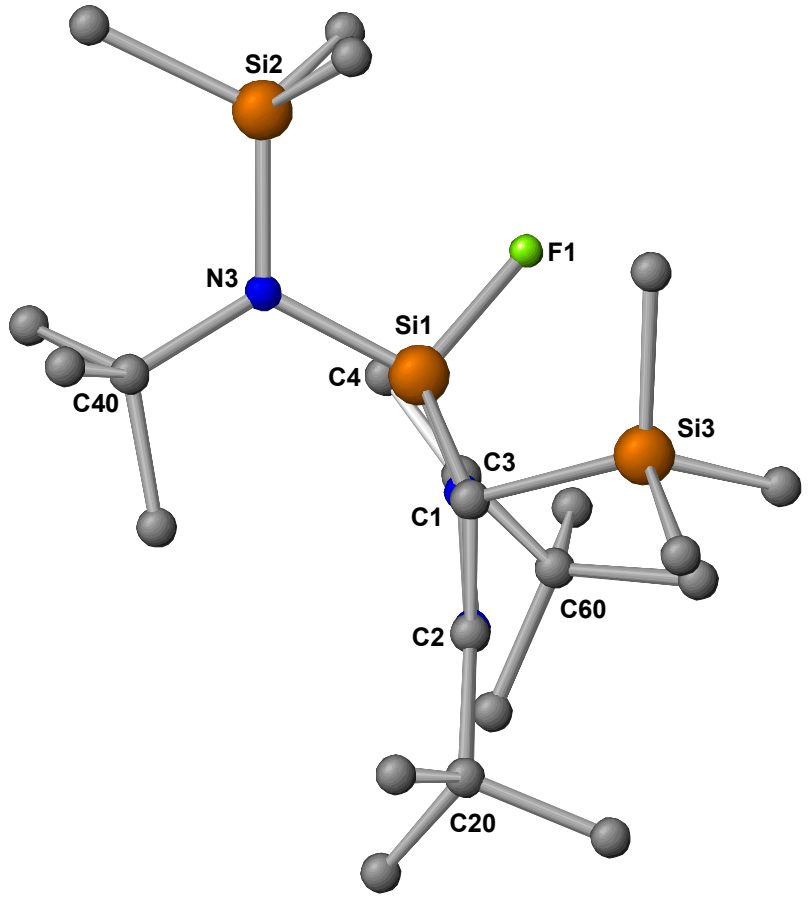

Blick auf die C1-C2-Bindung

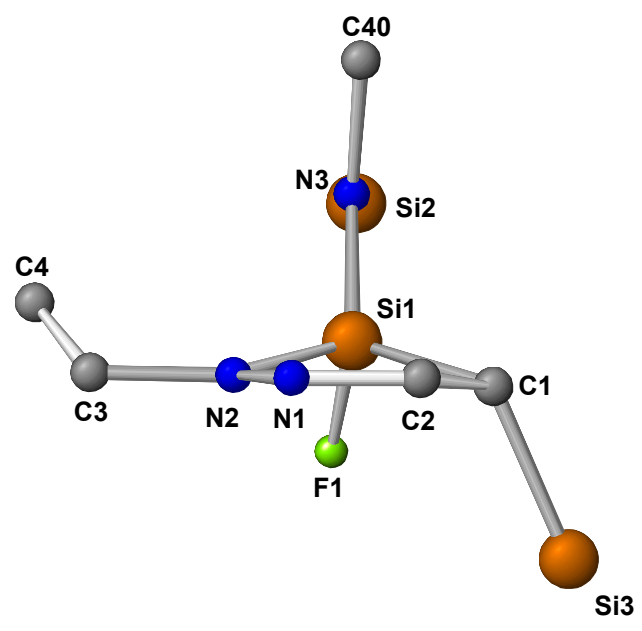

Blick auf die N1-C2-Bindung

Abbildung 28: Seitenansichten der Ringebene von Verbindung 39 


\section{Zusammenfassung}

Das Edukt der vorliegenden Arbeit ist das Bis(tert-butyl-methyl)ketazin I. Es besitzt zwei wesentliche Eigenschaften: Die Methylgruppen sind metallierbar und die tertButylgruppen üben einen stabilisierenden Effekt auf die Folgeprodukte aus. Ziel dieser Arbeit war die Synthese von unbekannten acyclischen und cyclischen Silylketazinen. Dabei sollte geprüft werden, in wieweit man die gewonnen Erkenntnisse über die verwandten Silylhydrazone auf die Ketazine übertragen kann.

Die Reaktion von monolithiiertem Bis(tert-butyl-methyl)ketazin I mit Chlorsilanen führt zu den acyclischen Silylketazinen 1, 2, 3, 7 und 8.<smiles>C/C(=N/N=C(\C)C(C)(C)C)C(C)(C)C</smiles><smiles>[Y16]/C(C[Si]([R])([R3])[R])=N\N=C(/C)C(C)(C)C</smiles>

$1,2,3,7,8$

\begin{tabular}{|c|c|c|c|c|c|}
\hline Verb. & $\mathbf{1}$ & $\mathbf{2}$ & $\mathbf{3}$ & $\mathbf{7}$ & $\mathbf{8}$ \\
\hline $\mathbf{R}_{\mathbf{1}}$ & $\mathrm{Me}$ & $\mathrm{Me}$ & $\mathrm{Me}$ & $\mathrm{Ph}$ & $\mathrm{Cl}$ \\
\hline $\mathbf{R}_{\mathbf{2}}$ & $\mathrm{Me}$ & $\mathrm{Me}$ & $\mathrm{Ph}$ & $\mathrm{Cl}$ & $\mathrm{Cl}$ \\
\hline $\mathbf{R}_{\mathbf{3}}$ & $\mathrm{Me}$ & $\mathrm{Cl}$ & $\mathrm{Cl}$ & $\mathrm{Cl}$ & $\mathrm{Cl}$ \\
\hline
\end{tabular}

Gleichung 44: Reaktion des Bis(tert-butyl-methyl)ketazins I zu acyclischen Silylketazinen

Chlorfunktionelle Mono(silyl)ketazine neigen bei der thermischen Aufarbeitung zur Chlorwasserstoffabspaltung und $\mathrm{zu}$ intra- und intermolekularen Cyclisierungsreaktionen.

Die Bis(ketazinyl)silane 4 und 5 konnten durch Reaktion des Monolithiumsalzes der Verbindung I bzw. 1 mit Dichlordimethylsilan im Verhältnis 2 : 1 dargestellt werden: 


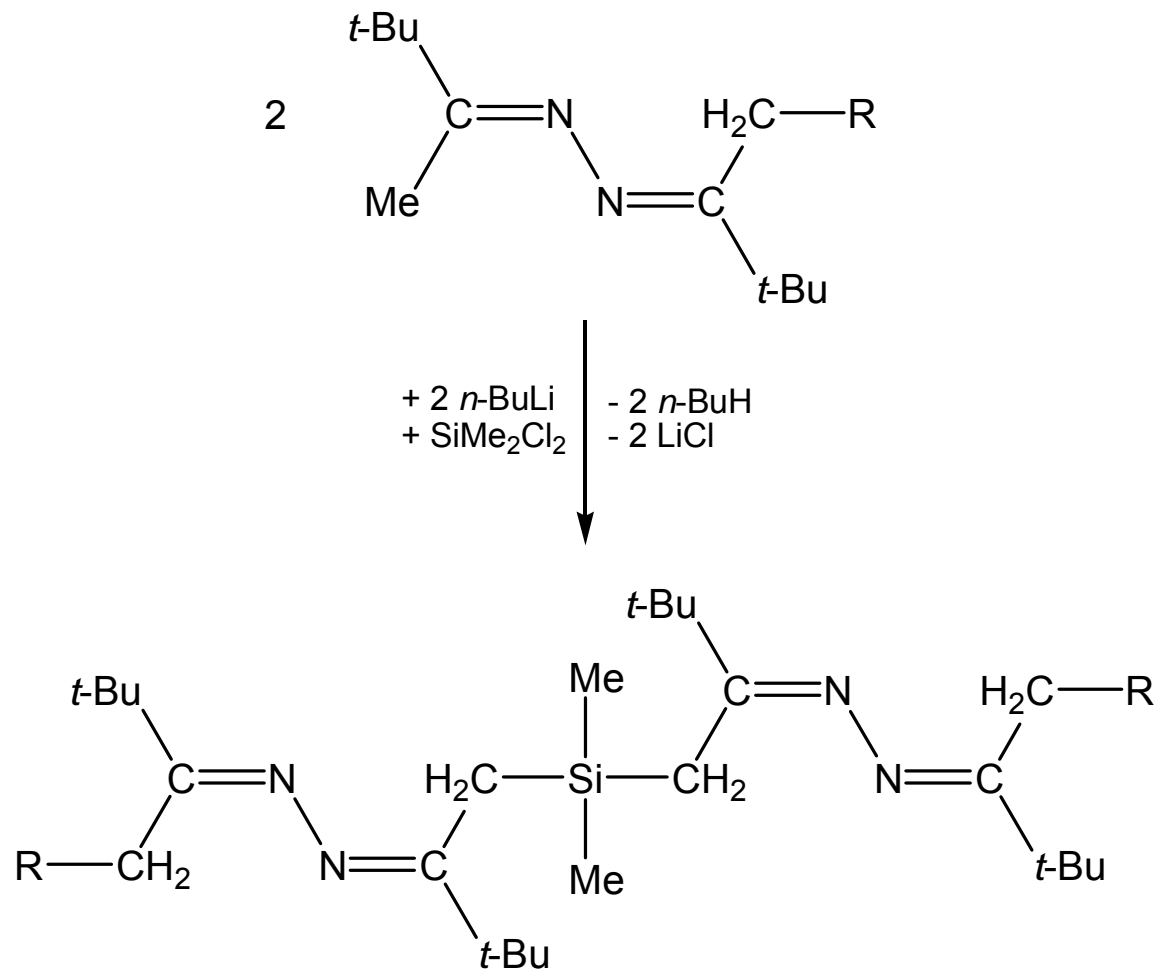

\begin{tabular}{|c|c|c|}
\multicolumn{4}{|c}{$\mathbf{4 , 5}$} \\
\hline Verb. & $\mathbf{4}$ & $\mathbf{5}$ \\
\hline R & H & SiMe $_{3}$ \\
\hline
\end{tabular}

Gleichung 45: Darstellung von Bis(ketazinyl)silanen

Durch Umsetzung der metallierten Verbindung I mit Di-tert-butyldichlorsilan entsteht das Lithium-Hydrazonid-THF-Addukt $\mathbf{6}$ nach noch ungeklärtem Reaktionsmechanismus.
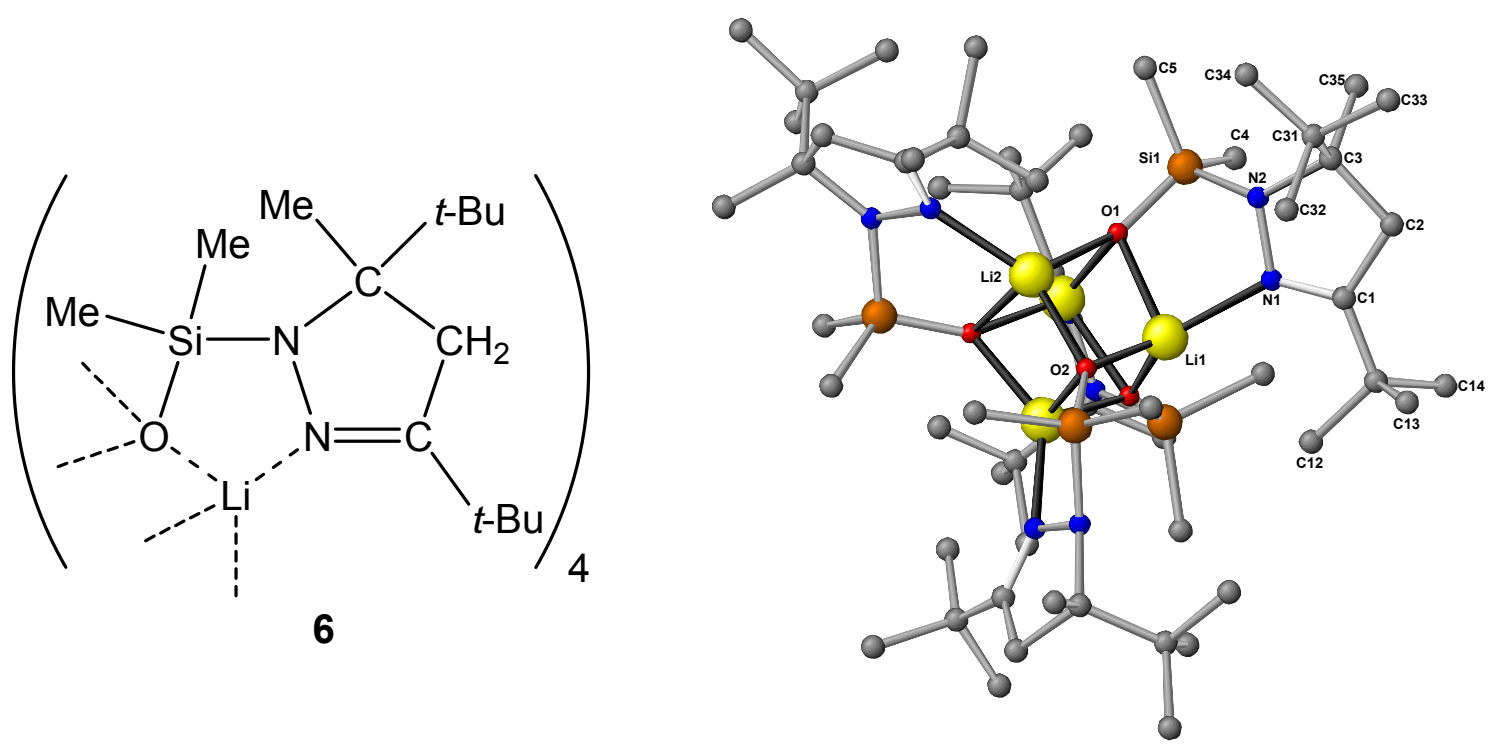

Abbildung 29: Ein Lithium-Hydrazonid-THF-Addukt 
Aus der Reaktion des Fluordimethylphenylsilans mit dem lithiierten Ketazin I bzw. der lithiierten monosilylierten Verbindung 1 erhält man die stabilen Verbindungen 9 und 10:

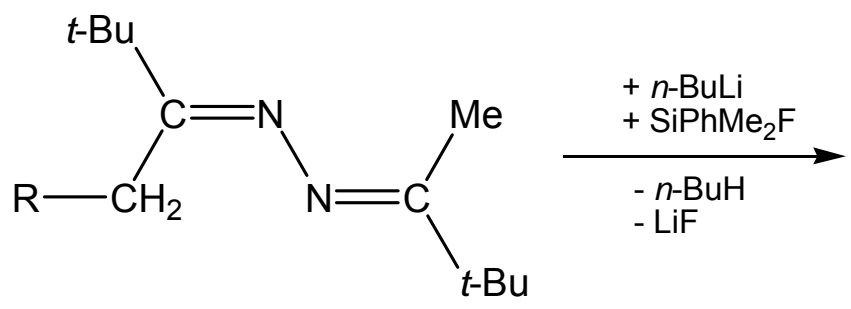

I bzw. 1<smiles>[R]C/C(=N/N=C(\C[Si](C)(C)c1ccccc1)C(C)(C)C)C(C)(C)C</smiles>

9,10

\begin{tabular}{|c|c|c|}
\hline Verb. & 9 & 10 \\
\hline $\mathbf{R}$ & $\mathrm{H}$ & $\mathrm{SiMe}_{3}$ \\
\hline
\end{tabular}

Gleichung 46: Reaktion des Monolithiumderivates von Verbindung I bzw. 1 mit

Fluordimethylphenylsilan

Die strukturisomeren Verbindungen 11 und 12 konnten bei der Umsetzung des monolithiierten Bis(tert-butyl-methyl)ketazins I mit Di-tert-butyldifluorsilan in unterschiedlichen Lösungsmitteln isoliert werden.<smiles>C=C(N1N=C(C(C)(C)C)C[Si]1(C(C)(C)C)C(C)(C)C)C(C)(C)C</smiles>

11<smiles>CC(C)(C)C(C)(C)C</smiles>

12

Abbildung 30: Die Strukturisomere 11 und 12

Für die Produktbildung von Verbindung 11 und 12 durch Eliminierung von zwei Lithiumfluorid wurden drei Reaktionswege dargelegt. 
Es wurde diskutiert, ob es sich bei Verbindung 12 auch um ein ebenfalls strukturisomeres, donor-stabilisiertes Silaethen handelt.<smiles>CC(C)(C)C(C)(C)C</smiles>

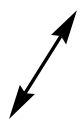<smiles>CC(C)(C)C(C)(C)C</smiles>

Silaethen
12

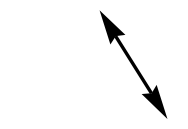

donor-stabilisiertes Silaethen

Schema 16: Mögliche Resonanzstrukturen von Verbindung 12

Die Synthese eines acyclischen fluorfunktionellen Silylketazins gelang durch die Reaktion der monolithiierten Verbindung I mit $N$-(Trifluorsilyl)-2-methyl- $N$-(trimethylsilyl)propan-2-amin.

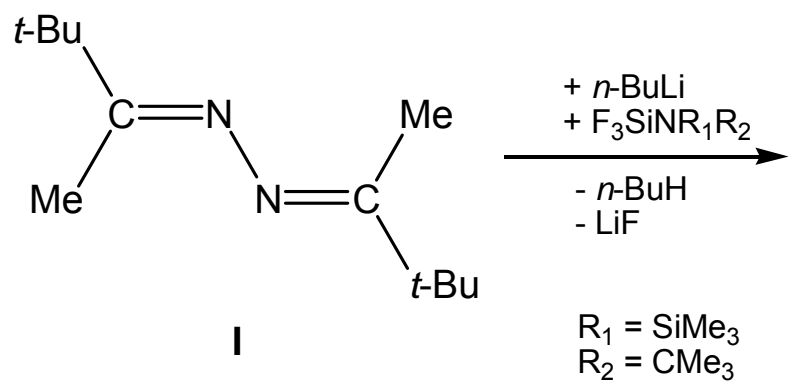

Gleichung 47: Darstellung des acyclischen Fuorsilylketazins 13<smiles>CC(C)(C)C(C)(C)C</smiles>

13

Von Verbindung 13 konnten durch Umkristallisation in $n$-Hexan Einkristalle für die Röntgenstrukturanalyse gewonnen werden. 


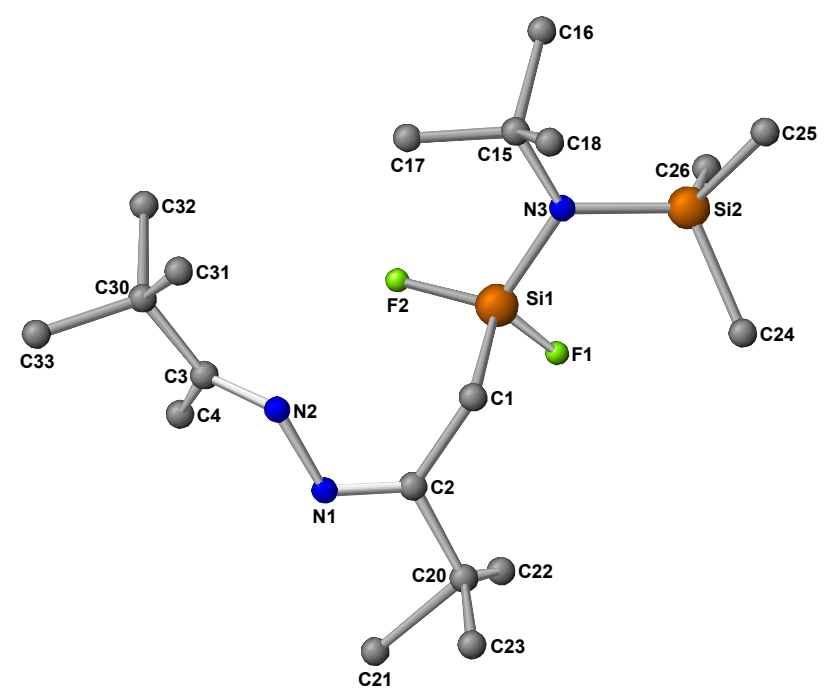

Abbildung 31: Kristallstruktur von Verbindung 13

Das erwartete Bis(ketazinyl)silan konnte in der Reaktion des Monolithiumderivates von Verbindung I mit Tetrafluorsilan im Verhältnis $2: 1$ nicht abgefangen werden. Es entsteht das Tris(ketazinyl)silan 14:

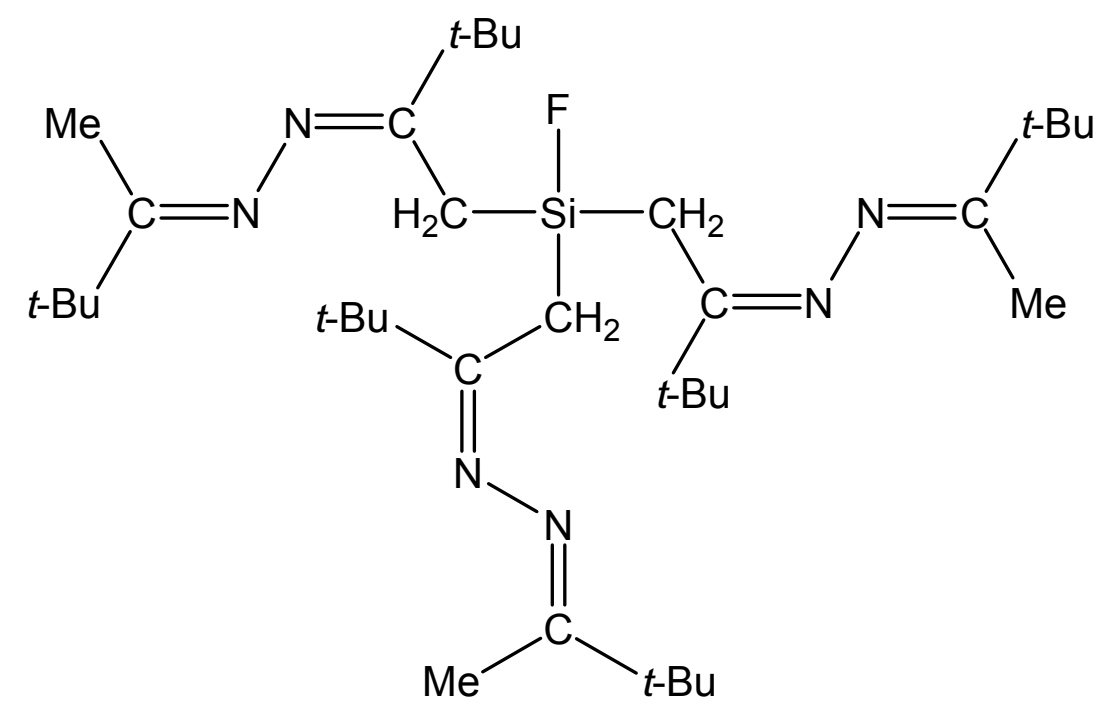

14

Abbildung 32: Struktur von Verbindung 14

Bei Umsetzungen der Dilithiumsalze des Bis(tert-butyl-methyl)ketazins I bzw. des Bis(tert-butyl-(trimethylsilyl)methyl)ketazins II mit Chlorsilanen im Verhältnis 1:1 bilden sich die cyclischen Silylketazine 15, 17, 18, 19 und 20: 


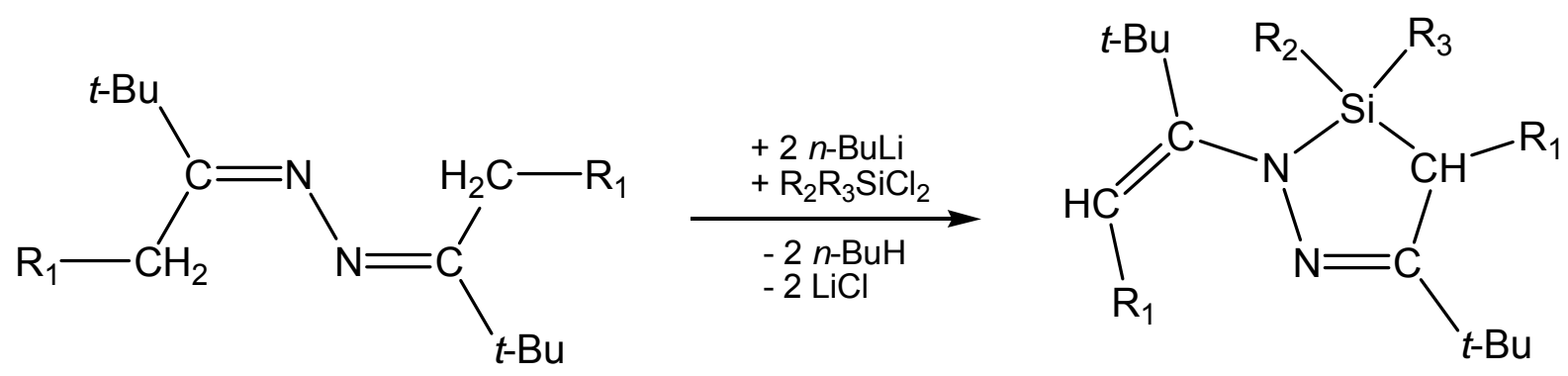

I bzw. II

$15,17,18,19,20$

\begin{tabular}{|c|c|c|c|c|c|}
\hline Verb. & $\mathbf{1 5}$ & $\mathbf{1 7}$ & $\mathbf{1 8}$ & $\mathbf{1 9}$ & $\mathbf{2 0}$ \\
\hline $\mathbf{R}_{\mathbf{1}}$ & $\mathrm{H}$ & $\mathrm{H}$ & $\mathrm{SiMe}_{3}$ & $\mathrm{H}$ & $\mathrm{SiMe}_{3}$ \\
\hline $\mathbf{R}_{\mathbf{2}}$ & $\mathrm{Me}$ & $\mathrm{Me}$ & $\mathrm{Me}$ & $\mathrm{Cl}$ & $\mathrm{Cl}$ \\
\hline $\mathbf{R}_{\mathbf{3}}$ & $\mathrm{Me}$ & $\mathrm{Ph}$ & $\mathrm{Ph}$ & $\mathrm{Cl}$ & $\mathrm{Cl}$ \\
\hline
\end{tabular}

Gleichung 48: Reaktion der dilithiierten Verbindung I bzw. II mit Chlorsilanen

Die Entstehung des Nebenproduktes 16 bei der Reaktion der dilithiierten Verbindung I mit Dichlordimethylsilan lässt sich nach dem vorgestellten Reaktionsweg $B$ (Schema 11, Seite 35) durch eine unvollständige Lithiierung und anschließende Addition von Chlorwasserstoff an die Doppelbindung des intermediären Imins erklären.<smiles>CC(C)(C)C(C)(C)C</smiles>

16

Abbildung 33: Struktur des Nebenproduktes 16

Durch Zugabe von Difluordiisopropylsilan zum Lithiumderivat von Verbindung I entsteht als Hauptprodukt die cyclische Verbindung 21 und zusätzlich die acyclische Verbindung 22: 


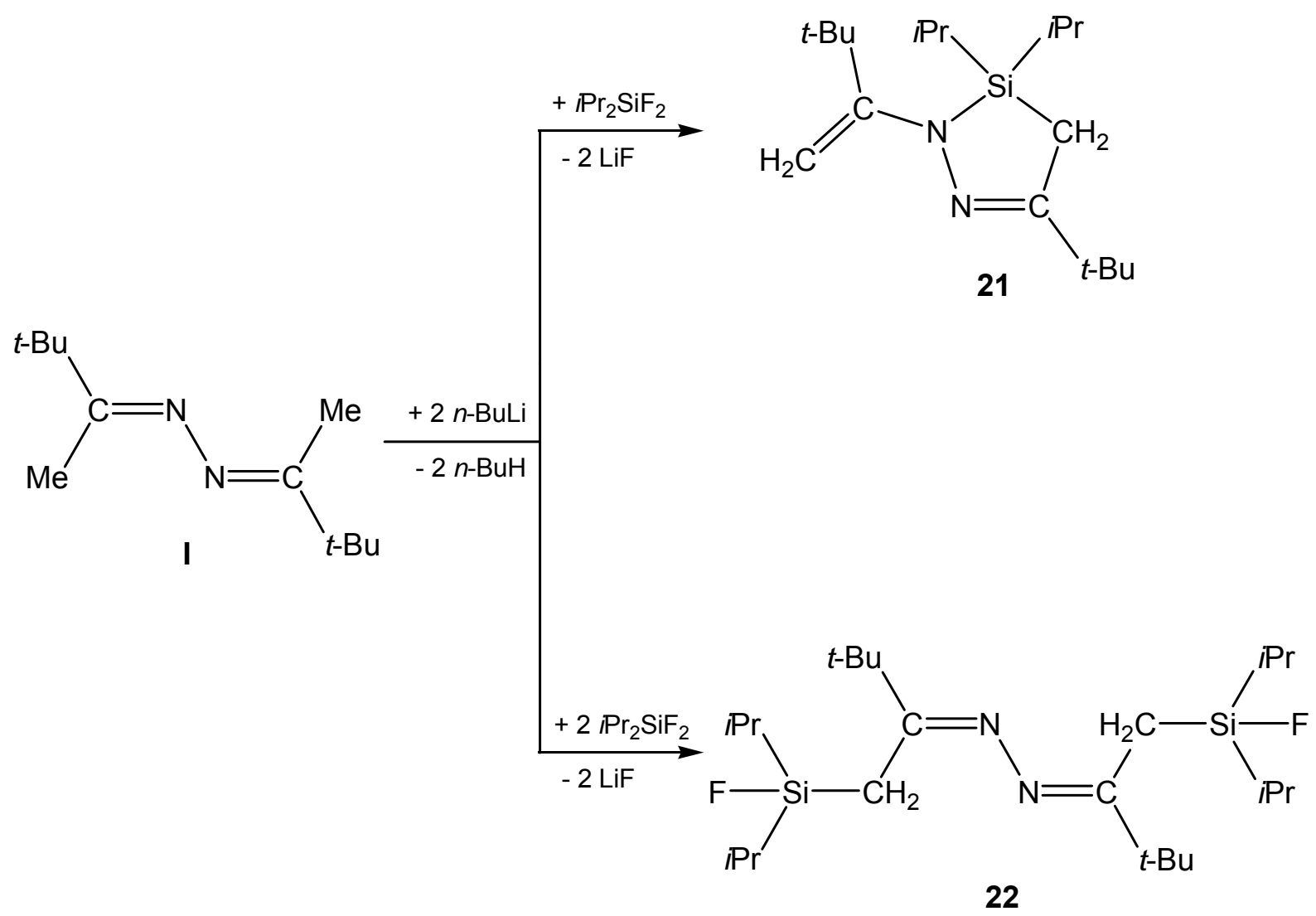

Gleichung 49: Reaktion des dilithiierten Ketazins I mit Difluordiisopropylsilan

Verbindung 22 wurde röntgenstrukturanalytisch vermessen und zeigt im Festkörper folgende Struktur:

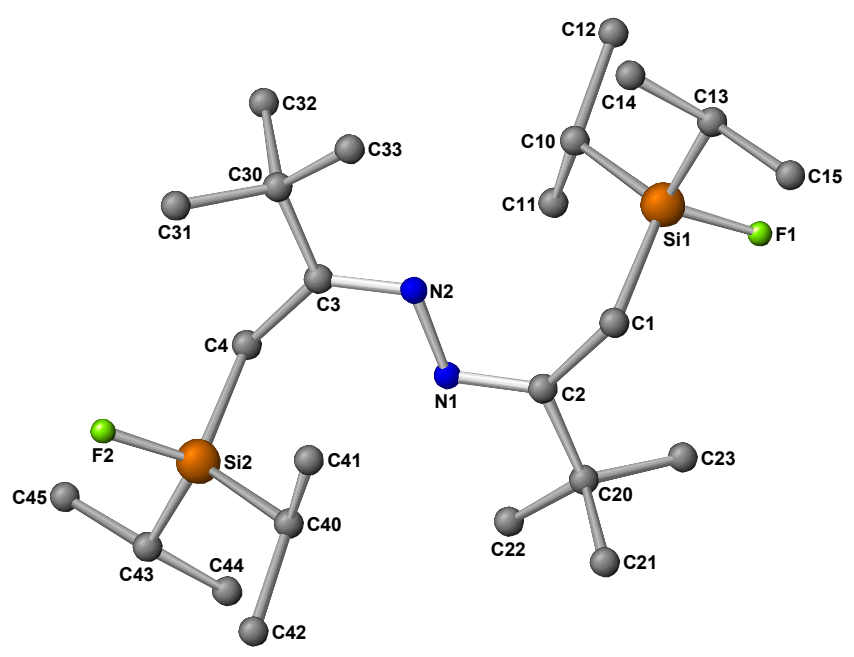

Abbildung 34: Kristallstruktur von Verbindung 22 
Bei Umsetzungen von Fluorsilanen mit lithiierten Hydrazonen erhält man Fluorsilylhydrazone. Durch erneute Reaktion mit tert-Butyllithium cyclisieren diese zu Diazasilacyclopentenen ${ }^{[43]}$. Versetzt man dilithiierte Ketazine mit Fluorsilanen, gelingt die Synthese in einem Reaktionsschritt:

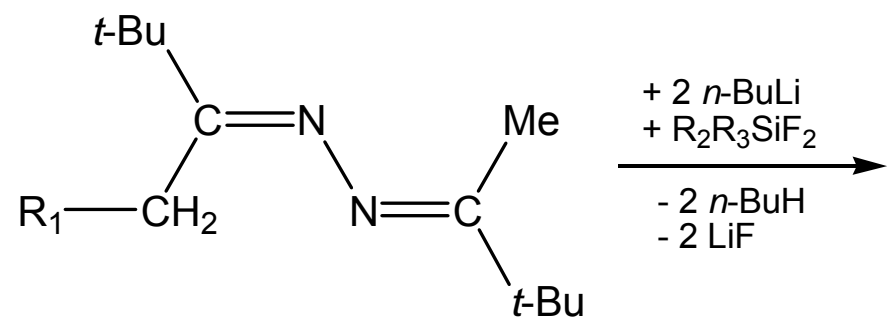

I bzw. 1<smiles>[R]C=C(N1N=C(C(C)(C)C)C[Si]1([R])[R])C(C)(C)C</smiles>

$23,25,26,27,28$

\begin{tabular}{|c|c|c|c|c|c|}
\hline Verb. & $\mathbf{2 3}$ & $\mathbf{2 5}$ & $\mathbf{2 6}$ & $\mathbf{2 7}$ & $\mathbf{2 8}$ \\
\hline $\mathbf{R}_{1}$ & $\mathrm{H}$ & $\mathrm{SiMe}_{3}$ & $\mathrm{H}$ & $\mathrm{H}$ & $\mathrm{H}$ \\
\hline $\mathbf{R}_{\mathbf{2}}$ & $\mathrm{Ph}$ & $t-\mathrm{Bu}$ & $t-\mathrm{Bu}$ & $\left(\mathrm{Me}_{3} \mathrm{Si}\right)_{2} \mathrm{~N}$ & $\left(\mathrm{Me}_{3} \mathrm{Si}\right)(t \mathrm{Bu}) \mathrm{N}$ \\
\hline $\mathbf{R}_{\mathbf{3}}$ & $t-\mathrm{Bu}$ & $t-\mathrm{Bu}$ & $\mathrm{F}$ & $\mathrm{F}$ & $\mathrm{F}$ \\
\hline
\end{tabular}

Gleichung 50: Synthese von Diazasilacyclopentenen

Das Diazasilepin 24 bildet sich bei thermischer Belastung in der Reaktion von Difluordi-tert-butylsilan und dem Dilithiumsalz von Verbindung I:

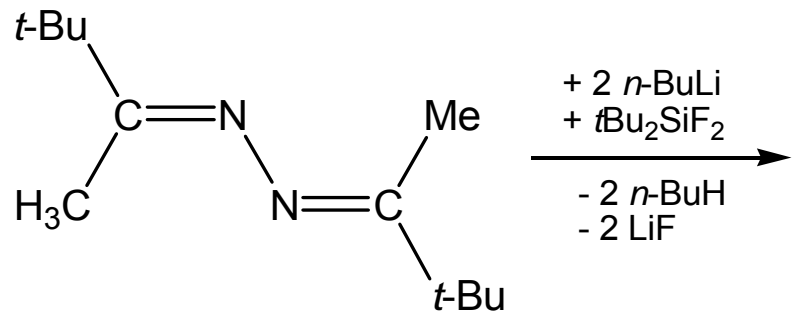

I<smiles>C[Z15](C)(C)C1=NN[Si](C(C)(C)C)(C(C)(C)C)CC(C(C)(C)C)=C1</smiles>

24

Gleichung 51: Bildung von Verbindung 24

Die Strukturen der Verbindungen $\mathbf{2 7}$ und $\mathbf{2 8}$ konnten durch Röntgenstrukturanalyse bestätigt werden: 


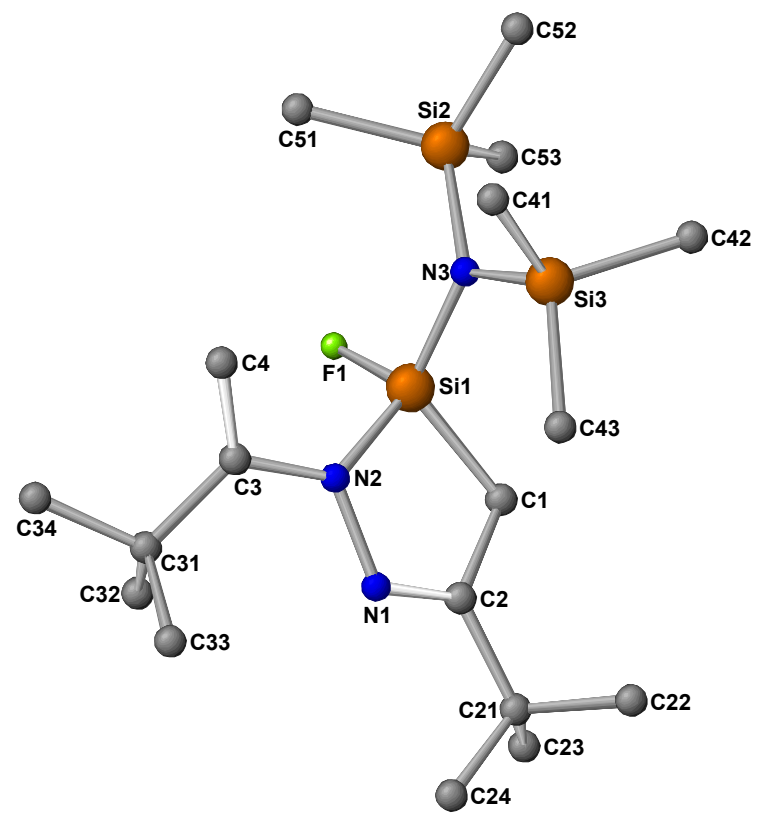

27

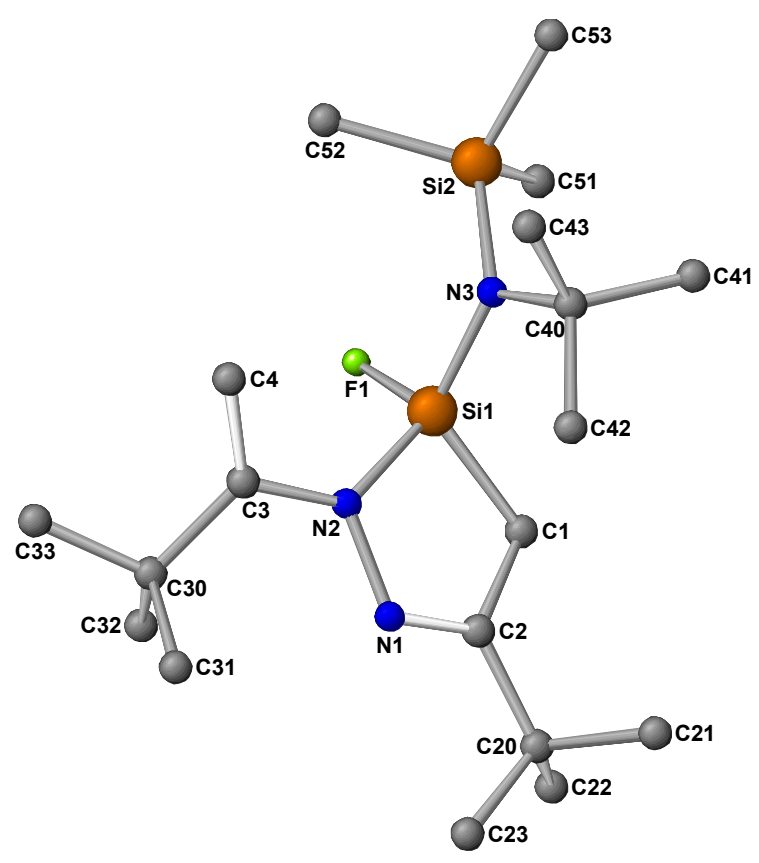

28

Abbildung 35: Kristallstrukturen der Verbindungen 27 und 28

Trifluorsilane reagieren mit dem dilithiierten Bis(tert-butyl-(trimethylsilyl)methyl)ketazin II im Verhältnis $1: 1$ zu den Diazasilacyclopentenen 29-32:

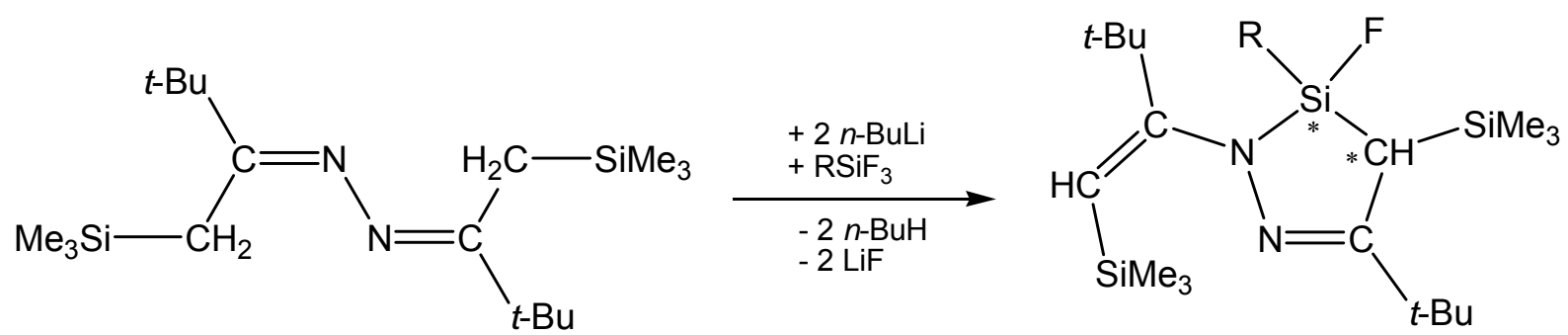

II

$29,30,31,32$

\begin{tabular}{|c|c|c|c|c|}
\hline Verb. & $\mathbf{2 9}$ & $\mathbf{3 0}$ & $\mathbf{3 1}$ & $\mathbf{3 2}$ \\
\hline $\mathbf{R}$ & $t-\mathrm{Bu}$ & $\mathrm{Ph}$ & $\left(\mathrm{Me}_{3} \mathrm{Si}\right)_{2} \mathrm{~N}$ & $\left(\mathrm{Me}_{3} \mathrm{Si}\right)(t-\mathrm{Bu}) \mathrm{N}$ \\
\hline
\end{tabular}

Gleichung 52: Synthese der Verbindungen 29-32

Die Verbindungen 29-32 fallen als Isomerengemische an, da sie zwei chirale Zentren besitzen und zudem an der terminalen Butyliden-Doppelbindung (E/Z)-Isomere bilden. 
Aus der Umsetzung von I bzw. II mit zwei Äquivalenten $n$-Butyllithium und anschließender Reaktion mit Tetrafluorsilan resultieren die Ketazin-Spirocyclen 33 und 34:

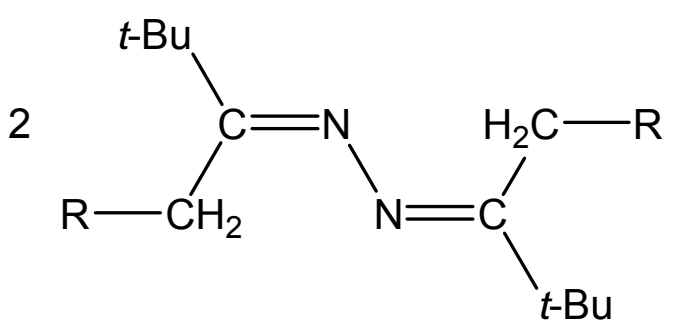

I bzw. II

$$
\begin{array}{l|l}
+4 n-B u L i & -4 n-B u H \\
+\mathrm{SiF}_{4} & -4 \mathrm{LiF}
\end{array}
$$

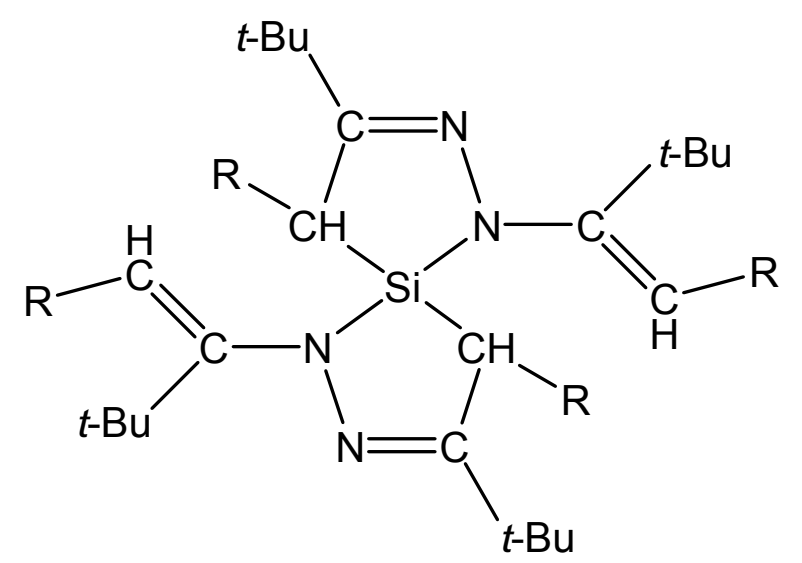

33, 34

\begin{tabular}{|c|c|c|}
\hline Verb. & $\mathbf{3 3}$ & $\mathbf{3 4}$ \\
\hline $\mathbf{R}$ & $\mathrm{H}$ & $\mathrm{SiMe}_{3}$ \\
\hline
\end{tabular}

Gleichung 53: Darstellung der Spirocyclen 33 und 34

Einkristalle konnten von Verbindung 34 durch Umkristallisation in $n$-Hexan gewonnen werden: 


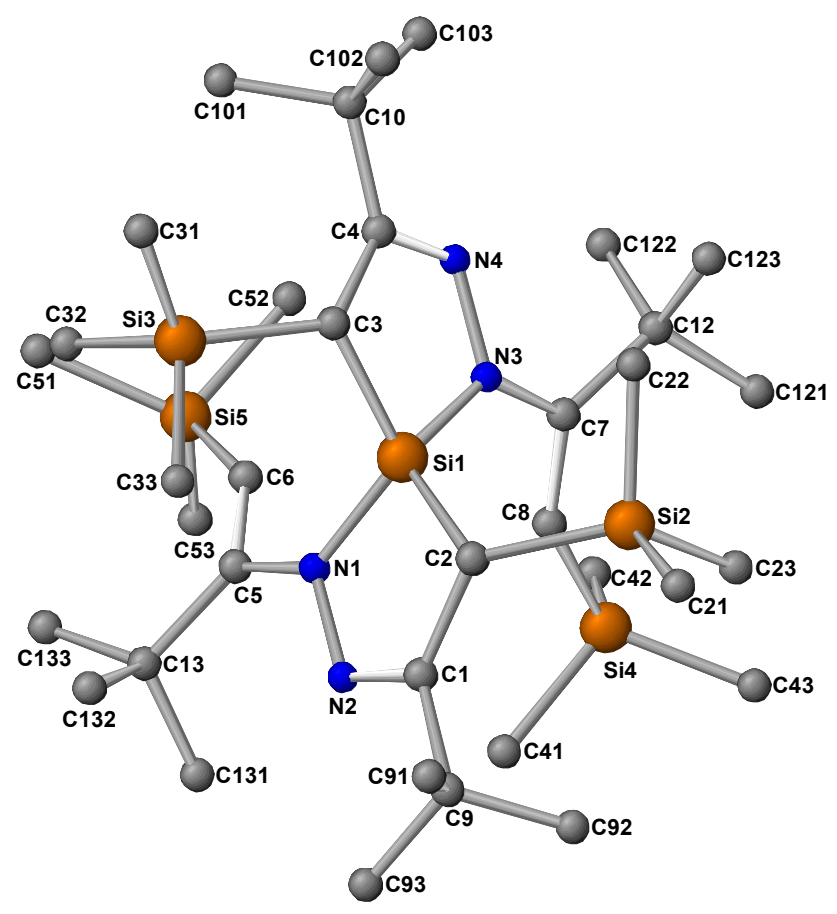

Abbildung 36: Struktur von 34 im Festkörper

Zur Strukturaufklärung wurde Verbindung 12 mit Methanol umgesetzt. Es entsteht quantitativ Verbindung 35:<smiles>CC(C)(C)C(C)(C)C</smiles>

12

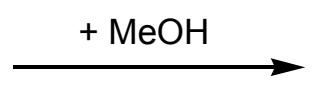

Gleichung 54: Reaktion von Verbindung 12 mit Methanol<smiles>CO[Si](C/C([As])=N\N=C(/C)C(C)(C)C)(C(C)(C)C)C(C)(C)[18O]</smiles>

35

Durch weiterführende Umsetzungen wurde allerdings festgestellt, dass Methanol die Si-N-Bindungen spaltet. Aus diesem Grund können keine endgültigen Schlussfolgerungen in Bezug auf ein mögliches donor-stabilisiertes Silaethen gezogen werden. 
Diazasilacyclopentene könnten geeignete Precursoren für die Stabilisierung einer $\mathrm{Si}=\mathrm{C}$-Doppelbindung darstellen. Sie weisen an Position vier ein acides Proton auf, das sich metallieren lässt. Durch Lithiumfluorid-Eliminierung könnten Silaethene gebildet werden. Dimerisationsprodukte von Silaethenen konnten bei den verwandten Hydrazonen nachgewiesen werden ${ }^{[50]}$.

Das hergestellte Diazasilacyclopenten $\mathbf{2 9}$ wurde mit verschiedenen Lithiumorganylen versetzt. Es kam aber nicht zu einer intramolekularen Salzeliminierung, sondern es konnten nur die Substitutionsprodukte 36-38 isoliert werden:

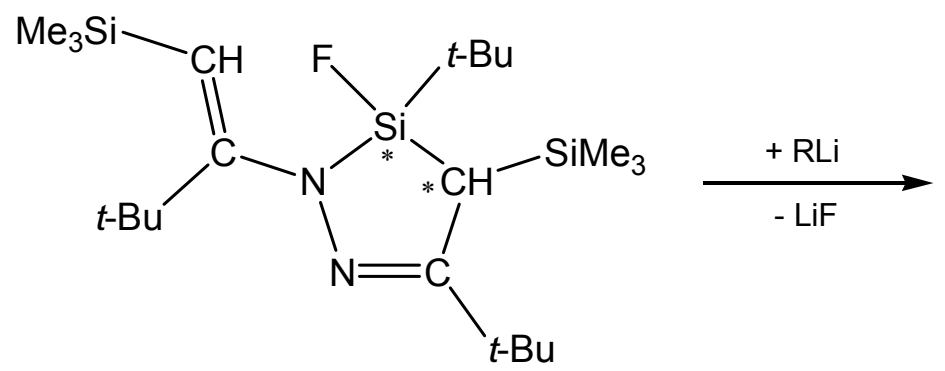

29<smiles>[R][Si]1(C(C)(C)C)C([As])C(C(C)(C)C)=NN1/C(=C/[SiH2]C(C)(C)C)C(C)(C)C</smiles>

$36,37,38$

\begin{tabular}{|c|c|c|c|}
\hline Verb. & $\mathbf{3 6}$ & $\mathbf{3 7}$ & $\mathbf{3 8}$ \\
\hline $\mathbf{R}$ & $\mathrm{Me}$ & $n-\mathrm{Bu}$ & $\mathrm{Ph}$ \\
\hline
\end{tabular}

Gleichung 55: Reaktion von Verbindung 29 mit Lithiumorganylen

Verbindung 29 wurde als Diastereomerengemisch im Verhältnis $2: 1$ erhalten. In den NMR-Spektren der Substitutionsprodukte 36, 37 und 38 ist jeweils nur noch ein Signalsatz zu finden. Eine Röntgenstrukturanalyse zeigt, dass Verbindung $\mathbf{3 8}$ als Enantiomerenpaar vorliegt. Von Verbindung $\mathbf{3 6}$ kristallisiert nur ein Isomer aus.

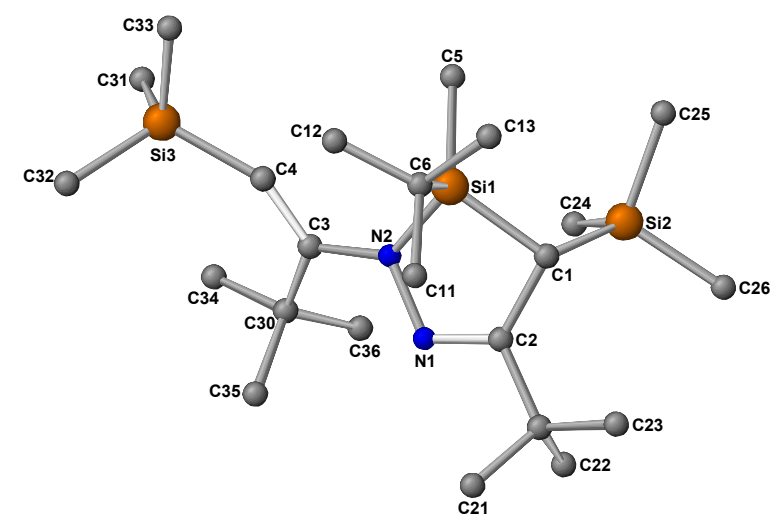

Abbildung 37: Kristallstruktur von Verbindung 36 

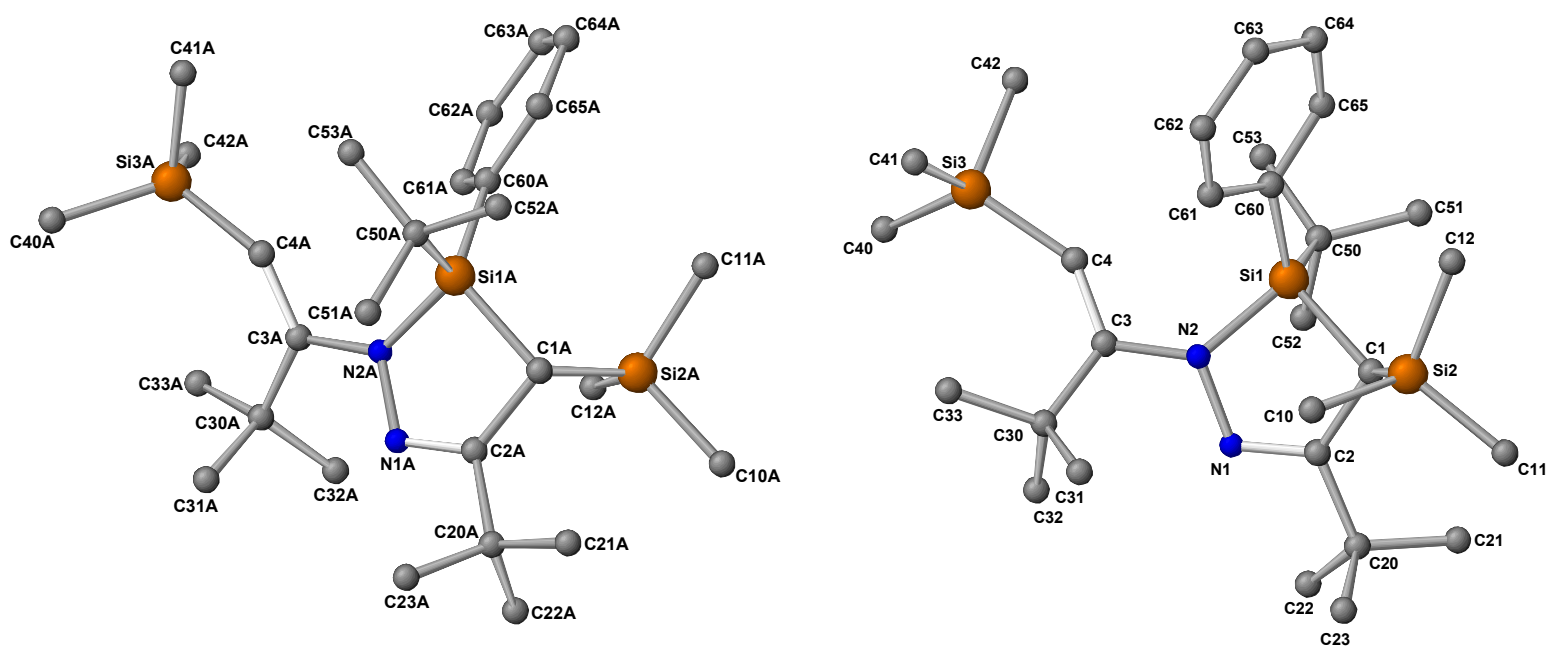

Abbildung 38: Kristallstrukturen des Enantiomerenpaars von Verbindung 38

Die selektive Bildung der Produkte könnte über einen $S_{N} 1$-Mechanismus erklärt werden. $\mathrm{Da}$ Silylkationen in der Siliciumchemie aber sehr selten vorkommen ${ }^{[70,71,72,73]}$, sind auch Mechanismen über einen fünf- oder sechsbindigen Übergangszustand des Siliciums in Erwägung zu ziehen.

Stabile Silaethene konnten durch thermische Salzeliminierung nach einem FluorChlor-Austausch isoliert werden ${ }^{[60]}$. Analog dazu wurde Verbindung 28 mit tertButyllithium im Verhältnis $1: 1$ und nach beendeter Butanabspaltung mit Chlortrimethylsilan versetzt. Es entsteht das Substitutionsprodukt 39, das durch Röntgenstrukturanalyse bestätigt wurde.<smiles>C=C(N1N=C(C(C)(C)C)C[Si]1(F)N(C(C)(C)C)C(C)(C)C)C(C)(C)C</smiles>

28

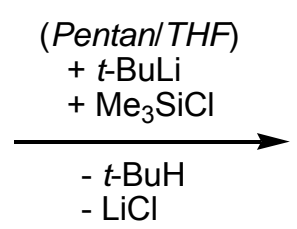

Gleichung 56: Reaktion von Verbindung 28 mit tert-Butyllithium<smiles>C=C(N1N=C(C(C)(C)C)[C@H](C)[Si]1(F)N(C(C)(C)C)C(C)(C)C)C(C)(C)C</smiles>

39 


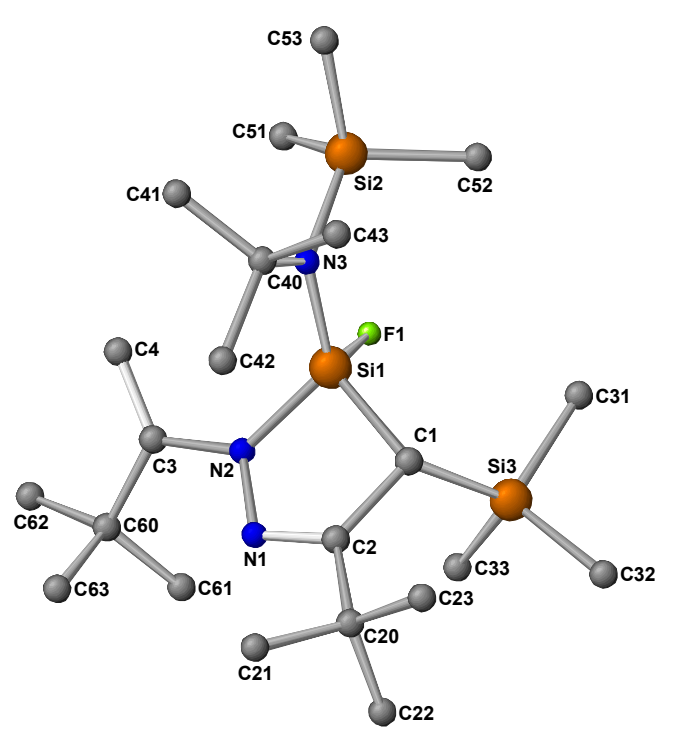

Abbildung 39: Kristallstruktur von Verbindung 39 


\section{Ausblick}

Die vorgestellten Silylketazine bieten weitere interessante Forschungsschwerpunkte. Durch die Synthese von neuen chlor- und fluorfunktionellen, acyclischen und cyclischen Silylketazinen könnten generelle Aussagen über Reaktionsmechanismen getroffen werden. Ausgedehnte Studien über den Einfluss der Reaktionsbedingungen, wie Solventien und Reaktionstemperatur, sind von besonderer Wichtigkeit. Umlithiierungen sollten an weiteren Silylketazinen erforscht und vor allem Lithiumderivate strukturell untersucht werden.

Eine Variation der Substituenten am Ketazin eröffnet die Möglichkeit das Reaktionsverhalten von Ketazinen mit Silanen thermodynamisch und kinetisch zu überprüfen. Eine zweckmäßige Verbindung könnte das Amino-tert-butylketazin sein. Es besitzt sowohl acide Wasserstoffatome als auch chelatisierende Stickstoffatome.

Um die Struktur der Verbindung 12 endgültig aufzuklären, sind weitere Reaktionen notwendig. Es bieten sich Cycloadditionen mit Butadien, Cyclopentadien, $N$ Trimethylsilylbenzophenonimin sowie tert-Butylazid an. Auch En-Reaktionen mit 2,3Dimethylbutadien, Isobuten und Aceton wären sinnvoll.

Die Verbindungen 19 und 20 könnten sich für die Darstellung von Silylenen eignen. In Anlehnung an WEST und DENK ${ }^{[69]}$ könnte eine Umsetzung mit geschmolzenem Kalium in THF zum Erfolg führen.

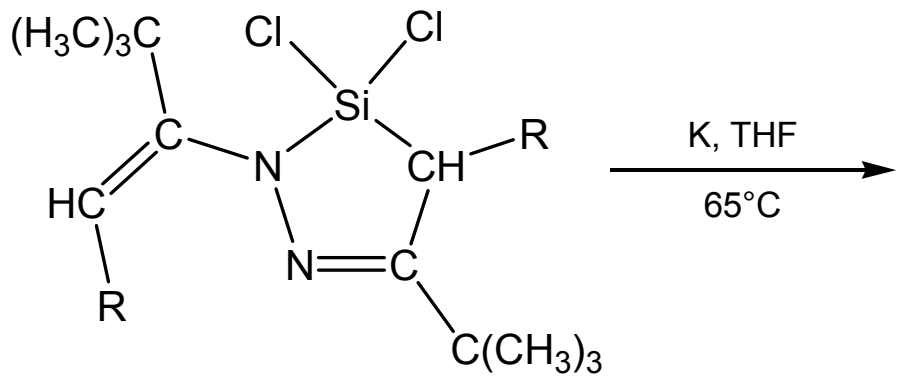<smiles>[R]C=C(N1N=C(C)C([R])[Si]1)C(C)(C)C</smiles>

19: $\mathrm{R}=\mathrm{H}$

20: $\mathrm{R}=\mathrm{SiMe}_{3}$ 
Eine zu 19 und 20 analoge Verbindung des Amino-tert-butylketazins wäre für die Synthese von stabilen Silylenen besonders vielversprechend.

Desweiteren könnten Substitutionen und Ringkopplungen sowohl an der gesättigten $\mathrm{CH}_{2}$-Gruppe als auch an den halogenfunktionellen Silylgruppen der dargestellten Diazasilacyclopentene durchgeführt werden.

Die Reaktion mit anderen Hauptgruppenelementverbindungen, wie z. B. Boranen und Phosphanen, könnte interessante Ergebnisse liefern.

Auch ergänzende Untersuchungen zu den gefundenen Isomeren stehen noch aus. Die Aufklärung des Reaktionsmechanismus, bei dem selektiv das kinetisch bevorzugte Enantiomerenpaar gebildet wird, erfordert noch intensive Forschungsarbeit. 


\section{Präparativer Teil}

\subsection{Allgemeine Arbeitsbedingungen}

Alle Reaktionen werden unter Luft- und Feuchtigkeitsausschluss in einer trockenen Inertgasatmosphäre (Argon oder Stickstoff) durchgeführt.

Soweit die benötigten Ausgangsverbindungen nicht im Handel erhältlich sind, werden sie nach den in der Literatur bekannten Vorschriften synthetisiert.

Die verwendeten Lösungsmittel werden nach den im Labor üblichen Methoden getrocknet und über Natriumdraht bzw. $\mathrm{KOH}$ aufbewahrt.

Das bei den Lithiierungen verwendete $n$-Butyllithium liegt als $15 \%$ ige bzw. $23 \%$ ige Lösung in $n$-Hexan vor, tert-Butyllithium als $15 \%$ ige Lösung in $n$-Pentan. Methyllithium wird als 5 \%ige Lösung in Ether, Phenyllithium als zwei molare Lösung in Cyclohexan / Ether eingesetzt.

Der Reaktionsfortschritt wird, soweit dies möglich ist, ${ }^{1} \mathrm{H}-$ bzw. ${ }^{19} \mathrm{~F}-\mathrm{NMR}$-spektroskopisch verfolgt.

Die Reinheit der dargestellten Verbindungen wird nach Möglichkeit gaschromatographisch sowie massen- und kernresonanzspektroskopisch überprüft.

\subsection{Darstellungsvorschriften für die Verbindungen 1 - 39}

\section{Verbindung 1}

In $300 \mathrm{~mL} n$-Hexan werden 0,5 mol (98 g) Bis(tert-butyl-methyl)ketazin gelöst und bei $0{ }^{\circ} \mathrm{C}$ mit $0,5 \mathrm{~mol}(139,2 \mathrm{~g}) \quad n$-Butyllithium (23\%ig in $n$-Hexan) versetzt. Das Reaktionsgemisch wird langsam zum Sieden erhitzt. Nach $4 \mathrm{~h}$ werden bei Raumtemperatur 0,55 mol (59,8 g) Chlortrimethylsilan hinzugegeben und weitere $2 \mathrm{~h}$ unter Rückfluss erhitzt. Das entstehende LiCl wird über eine Fritte abgetrennt und die verbleibende Lösung eingeengt. Durch fraktionierte Destillation bei vermindertem Druck (ca. 1 mbar) wird 1 analysenrein erhalten. 


\section{Verbindungen 2, 3, 7, 8}

Zu 0,1 mol (19,6 g) Bis(tert-butyl-methyl)ketazin in $200 \mathrm{~mL} n$-Hexan wird bei $0{ }^{\circ} \mathrm{C}$ die äquimolare Menge $(42,7 \mathrm{~g}) \quad n$-Butyllithium (15\%ig in $n$-Hexan) gegeben. Anschließend wird die Aufschlämmung $4 \mathrm{~h}$ zum Sieden erhitzt und nach dem Abkühlen in einen Tropftrichter überführt. 0,11 mol Chlorsilan (2: $14,2 \mathrm{~g} \mathrm{Me}_{2} \mathrm{SiCl}_{2}$; 3: $21,0 \mathrm{~g} \mathrm{PhMeSiCl}_{2}$; 7: 23,3 $\mathrm{g} \mathrm{PhSiCl}_{3}$; 8: $18,7 \mathrm{~g} \mathrm{SiCl}_{4}$ ) in $50 \mathrm{~mL}$ Hexan werden auf - $60^{\circ} \mathrm{C}$ gekühlt und das lithiierte Ketazin langsam zugetropft. Das Reaktionsgemisch wird über Nacht auf Raumtemperatur gebracht und $2 \mathrm{~h}$ zum Sieden erhitzt. Das Rohprodukt wird vom entstandenen $\mathrm{LiCl}$ im Vakuum (2, 8 ca. 1 mbar; 3, 7 ca. 0,01 mbar) abgetrennt. Durch fraktionierte Destillation erhält man die Verbindungen 2, 3, 7 und 8.

\section{Verbindungen 4, 5}

0,1 mol (19,6 g) Bis(tert-butyl-methyl)ketazin für Verbindung 4 bzw. 0,1 mol $(26,8 \mathrm{~g})$ von Verbindung 1 werden mit $0,1 \mathrm{~mol} \quad(27,8 \mathrm{~g}) \quad n$-Butyllithium $(23 \%$ ig in $n$-Hexan) in $200 \mathrm{~mL} n$-Hexan bei $0^{\circ} \mathrm{C}$ lithiiert, indem die Lösung dann $3 \mathrm{~h}$ in der Siedehitze rührt. 0,06 mol (7,7 g) $\mathrm{Me}_{2} \mathrm{SiCl}_{2}$ werden zu der warmen Reaktionslösung hinzugegeben, die für weitere $5 \mathrm{~h}$ auf Siedetemperatur erhitzt wird. Durch Zentrifugieren trennt man das entstandene $\mathrm{LiCl}$ von der überstehenden Lösung. Die Lösung wird eingeengt und die Verbindungen $\mathbf{4}$ und $\mathbf{5}$ durch fraktionierte Destillation (ca. 0,01 mbar) sauber erhalten.

\section{Verbindung 6}

Mit $200 \mathrm{~mL} n$-Hexan werden 0,1 mol (19,6 g) Bis(tert-butyl-methyl)ketazin in Lösung gebracht. Nach Zugabe von 0,1 mol (42,7 g) $n$-Butyllithium (15\%ig in $n$-Hexan) und $2 \mathrm{~h}$ Sieden unter Rückfluss ist die Metallierung abgeschlossen und das Reaktionsgemisch wird auf $0{ }^{\circ} \mathrm{C}$ abgekühlt. $0,1 \mathrm{~mol}(21,3 \mathrm{~g})$ Di-tert-butyldichlorsilan in $50 \mathrm{~mL}$ THF werden hinzugetropft und das Reaktionsprodukt für $6 \mathrm{~h}$ am Sieden gehalten. Das Rohprodukt wird zentrifugiert, eingeengt und dann in einem $n$-Hexan / THF-Gemisch umkristallisiert. 


\section{Verbindungen 9, 13}

In $200 \mathrm{~mL} n$-Hexan werden $0,1 \mathrm{~mol}$ (19,6 g) Bis(tert-butyl-methyl)ketazin gelöst und mit 0,1 mol (42,7 g) $n$-Butyllithium (15\%ig in $n$-Hexan) während $2 \mathrm{~h}$ in der Siedehitze metalliert. Für Verbindung

9 wird das Monolithiumsalz bei Raumtemperatur zu 0,1 mol $(15,4 \mathrm{~g})$ Fluordimethylphenylsilan in $50 \mathrm{~mL}$ Hexan $\left(-60^{\circ} \mathrm{C}\right)$ zugetropft.

13 wird das Monolithiumsalz auf $-60^{\circ} \mathrm{C}$ gekühlt und zu $0,11 \mathrm{~mol}(25,2 \mathrm{~g}) \mathrm{N}$ (trifluorsilyl)-2-methyl- $N$-(trimethylsilyl)propan-2-amin in $50 \mathrm{~mL}$ THF $\left(-60^{\circ} \mathrm{C}\right)$ hinzugetropft.

Die Lösung wird über Nacht auf Raumtemperatur erwärmt und für $8 \mathrm{~h}$ zum Sieden erhitzt. Die Trennung vom LiF erfolgt durch Umkondensation und die folgende Aufreinigung durch Destillation im Vakuum (ca. 0,01 mbar). Verbindung 13 wurde in $n$-Hexan umkristallisiert.

\section{Verbindung 10}

$0,1 \mathrm{~mol}(26,8 \mathrm{~g})$ von Verbindung 1 werden in $200 \mathrm{~mL} n$-Hexan gelöst und mit der äquimolaren Menge (27,8 g) $n$-Butyllithium (23\%ig in $n$-Hexan) zur Reaktion gebracht. Nach $3 \mathrm{~h}$ Sieden unter Rückfluss wird das Monolithiumsalz auf $-60^{\circ} \mathrm{C}$ abgekühlt und $0,1 \mathrm{~mol}(15,4 \mathrm{~g})$ Fluordimethylphenylsilan in $50 \mathrm{~mL}$ Hexan hinzugegeben. Das Gemisch wird über $6 \mathrm{~h}$ auf Raumtemperatur erwärmt und $8 \mathrm{~h}$ unter Rückfluss erhitzt. Nach Abtrennen des Lithiumfluorids im Vakuum (ca. 0,01 mbar) erhält man die reine Verbindung 10 durch Destillation im Vakuum.

\section{Verbindungen 11, 12}

$0,075 \mathrm{~mol}(14,7 \mathrm{~g})$ Bis(tert-butyl-methyl)ketazin werden bei Raumtemperatur mit $0,075 \mathrm{~mol}$ (32 g) $n$-Butyllithium (15\%ig in $n$-Hexan) in $200 \mathrm{~mL} n$-Hexan lithiiert. Die Suspension wird $2 \mathrm{~h}$ auf Siedetemperatur gehalten und wieder auf $0{ }^{\circ} \mathrm{C}$ abgekühlt, bevor $0,075 \mathrm{~mol}(13,5 \mathrm{~g})$ Di-tert-butyldifluorsilan hinzugegeben werden. Anschließend wird $5 \mathrm{~h}$ unter Rückfluss erhitzt und das entstandene LiF durch Umkondensation im Vakuum (ca. 0,01 mbar) abgetrennt. Nach Destillation erhält man ein Gemisch der beiden isomeren Verbindungen 11 und 12.

Löst man das Di-tert-butyldifluorsilan vor dem Zutropfen in $50 \mathrm{~mL}$ THF, erhält man nur Verbindung 12. 
Verbindung 11 ist ebenfalls über das Dilithiumsalz des Bis(tert-butyl-methyl)ketazins zugänglich.

\section{Verbindung 14}

$0,2 \mathrm{~mol} \quad(39,2 \mathrm{~g}) \quad$ Bis(tert-butyl-methyl)ketazin werden in $250 \mathrm{~mL} \quad n$-Hexan aufgenommen und mit 0,2 mol (85,4 g) $n$-Butyllithium (15\%ig in $n$-Hexan) versetzt. Danach wird $3 \mathrm{~h}$ bis zur völligen Butanabspaltung erhitzt. Das Lithiumsalz wird auf - $70{ }^{\circ} \mathrm{C}$ gekühlt und $0,1 \mathrm{~mol}(10,4 \mathrm{~g})$ Tetrafluorsilan eingeleitet. Das Gemisch wird über Nacht auf Raumtemperatur erwärmt und durch Zentrifugation vom entstandenen Lithiumfluorid abgetrennt. Die Lösung wird eingeengt und die Verbindung 14 durch fraktionierte Destillation (ca. 0,01 mbar) gereinigt.

\section{Verbindungen 15, 16, 17, 19}

Jeweils $0,1 \mathrm{~mol}(19,6 \mathrm{~g})$ Bis(tert-butyl-methyl)ketazin werden bei Raumtemperatur in $200 \mathrm{~mL} n$-Hexan gelöst und mit der doppelt äquimolaren Menge $(55,6 \mathrm{~g})$ $n$-Butyllithium (23\%ig in $n$-Hexan) versetzt. Nach $4 \mathrm{~h}$ Sieden unter Rückfluss ist die Metallierung abgeschlossen. Das Lithiumsalz wird für Verbindung

15 bei $-50{ }^{\circ} \mathrm{C}$ langsam zu einer Lösung von $0,11 \mathrm{~mol}(14,2 \mathrm{~g}) \mathrm{Me}_{2} \mathrm{SiCl}_{2}$ in $50 \mathrm{~mL}$ $n$-Hexan $\left(-50^{\circ} \mathrm{C}\right)$ zugetropft. Die Suspension wird über Nacht aufgetaut.

16 bei $30^{\circ} \mathrm{C}$ zu einer Lösung von $0,11 \mathrm{~mol}(14,2 \mathrm{~g}) \mathrm{Me}_{2} \mathrm{SiCl}_{2}$ in $50 \mathrm{~mL} n$-Hexan getropft.

17 bei Raumtemperatur langsam zu einer Lösung von $0,11 \mathrm{~mol} \mathrm{PhMeSiCl}_{2}$ in $50 \mathrm{~mL}$ THF $\left(-70^{\circ} \mathrm{C}\right)$ gegeben und innerhalb $5 \mathrm{~h}$ auf Raumtemperatur gebracht.

19 bei Raumtemperatur zu einer Lösung von $0,1 \mathrm{~mol}(17 \mathrm{~g}) \mathrm{SiCl}_{4}$ in $50 \mathrm{~mL}$ $n$-Hexan $\left(-60^{\circ} \mathrm{C}\right)$ zugetropft. Die Suspension wird langsam aufgetaut.

Anschließend wird die jeweilige Lösung $3 \mathrm{~h}$ zum Sieden erhitzt. Das Rohprodukt wird durch Umkondensation im Vakuum (15, 16, 19 ca. 1 mbar; 17 ca. 0,01 mbar) vom entstandenen $\mathrm{LiCl}$ abgetrennt und destillativ gereinigt.

\section{Verbindungen 18, 20}

In $100 \mathrm{~mL} n$-Hexan und $100 \mathrm{~mL}$ THF werden 0,1 mol (34,1 g) 1,4-Di-tert-butyl-1,4bis(trimethylsilylmethyl)ketazin gelöst und mit der doppelt äquimolaren Menge (55,6 g) n-Butyllithium (23 \%ig in $n$-Hexan) zur Reaktion gebracht. Nach 4 h Erhitzen 
unter Rückfluss wird die Lösung auf Raumtemperatur abgekühlt. Das metallierte Ketazin wird zu 0,11 mol Chlorsilan in $50 \mathrm{~mL}$ THF $\left(-60^{\circ} \mathrm{C}\right)$ zugetropft $(18: 21,0 \mathrm{~g}$ $\mathrm{PhMeSiCl}_{2} ; 20$ : 18,7 $\mathrm{g} \mathrm{SiCl}_{4}$ ), langsam wieder erwärmt und $6 \mathrm{~h}$ auf Siedetemperatur erhitzt. Entstandenes $\mathrm{LiCl}$ wird abzentrifugiert, das Rohprodukt eingeengt und destilliert (ca. 0,01 mbar).

\section{Verbindungen 21, 22, 23, 26, 27, 28}

Man metalliert jeweils $0,1 \mathrm{~mol}(19,6 \mathrm{~g})$ Bis(tert-butyl-methyl)ketazin in $250 \mathrm{~mL}$ $n$-Hexan, indem man bei Raumtemperatur 0,2 mol $(55,6 \mathrm{~g}) n$-Butyllithium (23 \%ig in $n$-Hexan) zutropft. Nach $4 \mathrm{~h}$ unter Sieden wird das Reaktionsgemisch auf $-60{ }^{\circ} \mathrm{C}$ abgekühlt und $\mathrm{zu} 0,11 \mathrm{~mol}$ ebenfalls auf $-60^{\circ} \mathrm{C}$ gekühltem Fluorsilan getropft (21 / 22: 16,7 g (i-Pr) $)_{2} \mathrm{SiF}_{2} ;$ 23: 22,0 g PhtBuSiF $;$ 26: 15,6 g tBuSiF $;$; 27: 27,0 g $\left(\mathrm{Me}_{3} \mathrm{Si}\right)_{2} \mathrm{NSiF}_{3} ; 28:$ 25,2 g $\left.\left(\left(\mathrm{Me}_{3} \mathrm{Si}\right)(t \mathrm{Bu}) \mathrm{N}\right) \mathrm{SiF}_{3}\right)$. Die Reaktionslösung wird über Nacht auf Raumtemperatur gebracht und danach $1 \mathrm{~h}$ auf Siedetemperatur erhitzt. Entstandenes LiF wird durch Umkondensation im Vakuum (ca. 0,01 mbar) abgetrennt und die Verbindungen durch fraktionierte Destillation gereinigt.

Verbindung 22 kristallisierte in der Vorlage aus. Röntgentaugliche Kristalle von Verbindung 27 und 28 konnten durch Umkristallisation in $n$-Hexan erhalten werden.

\section{Verbindung 24}

$0,13 \mathrm{~mol}(25,5 \mathrm{~g})$ Bis(tert-butyl-methyl)ketazin in $250 \mathrm{~mL} n$-Hexan werden mit 0,26 mol (110,9 g) $n$-Butyllithium (15\%ig in $n$-Hexan) versetzt und $4 \mathrm{~h}$ bis zur völligen Butanabspaltung lithiiert. 0,13 mol $(23,4 \mathrm{~g})$ Di-tert-butyldifluorsilan werden zugetropft und das Lösungsmittel abdestilliert. Das Gemisch wird für $3 \mathrm{~h}$ auf $128^{\circ} \mathrm{C}$ gehalten. LiF wird durch Umkondensation im Vakuum (ca. 0,01 mbar) abgetrennt und Verbindung 24 durch Destillation im Vakuum gereinigt.

\section{Verbindung 25}

$0,1 \mathrm{~mol}(26,8 \mathrm{~g})$ von Verbindung 1 werden in $250 \mathrm{~mL} n$-Hexan gelöst und mit 0,2 mol $(85,4 \mathrm{~g}) \quad n$-Butyllithium (15\%ig in $n$-Hexan) metalliert. Nachdem das Reaktionsgemisch $3 \mathrm{~h}$ auf Siedetemperatur gehalten wurde, wird es in einen Tropftrichter überführt und auf $-65^{\circ} \mathrm{C}$ gekühlt. Das Dilithiumsalz wird nun zu $0,1 \mathrm{~mol}$ $(18,0 \mathrm{~g})$ Di-tert-butyldifluorsilan in $50 \mathrm{~mL}$ THF $\left(-65^{\circ} \mathrm{C}\right)$ zugetropft und noch $15 \mathrm{~min}$ in der Kälte gerührt. Danach wird das Gemisch über $5 \mathrm{~h}$ auf Raumtemperatur erwärmt 
und $1 \mathrm{~h}$ unter Rückfluss erhitzt. Das Reaktionsgemisch wird eingeengt, das LiF im Vakuum (ca. 1 mbar) abgetrennt und das Rohprodukt fraktioniert destilliert.

\section{Verbindungen 29, 30, 31, 32}

Jeweils $0,1 \mathrm{~mol}(34,1 \mathrm{~g})$ 1,4-Di-tert-butyl-1,4-bis(trimethylsilylmethyl)ketazin werden in $100 \mathrm{~mL} n$-Hexan und $100 \mathrm{~mL}$ THF gelöst und mit $0,2 \mathrm{~mol}(55,6 \mathrm{~g}) n$-Butyllithium (23\%ig in $n$-Hexan) während $4 \mathrm{~h}$ in der Siedehitze dilithiiert. Die Reaktionslösung wird auf $-60^{\circ} \mathrm{C}$ gekühlt und zu $0,11 \mathrm{~mol}$ Fluorsilan in $50 \mathrm{~mL}$ THF $\left(-60^{\circ} \mathrm{C}\right)$ zugetropft (29: 15,6 g tBuSiF $;$ 30: 17,8 g PhSiF $;$ 31: 27,0 g $\left(\mathrm{Me}_{3} \mathrm{Si}_{2}\right)_{2} \mathrm{NSiF}_{3}$; 32: $\left.25,2 \mathrm{~g}\left(\left(\mathrm{Me}_{3} \mathrm{Si}\right)(t \mathrm{Bu}) \mathrm{N}\right) \mathrm{SiF}_{3}\right)$. Die Lösung wird über Nacht auf Raumtemperatur erwärmt, bevor sie $5 \mathrm{~h}$ unter Rückfluss erhitzt wird. Die Trennung des Rohproduktes vom LiF erfolgt durch Umkondensation und anschließend findet die Reinigung durch Destillation im Vakuum (29 ca. 1 mbar; 30, 31, 32 ca. 0,01 mbar) statt.

\section{Verbindungen 33, 34}

Es werden $0,05 \mathrm{~mol}(9,8 \mathrm{~g})$ Bis(tert-butyl-methyl)ketazin für Verbindung 33 bzw. 0,05 mol (17,0 g) 1,4-Di-tert-butyl-1,4-bis(trimethylsilylmethyl)ketazin für Verbindung 34 in $150 \mathrm{~mL} n$-Hexan gelöst und bei Raumtemperatur mit $0,1 \mathrm{~mol}(27,8 \mathrm{~g})$ $n$-Butyllithium (23\%ig in $n$-Hexan) dilithiiert. Nach beendeter Butanabspaltung wird das Gemisch wieder auf Raumtemperatur gebracht. Über einen Tropftrichter wird das Dilithiumsalz bei $-70{ }^{\circ} \mathrm{C}$ zu $0,05 \mathrm{~mol}(5,2 \mathrm{~g}) \mathrm{SiF}_{4}$ in $50 \mathrm{~mL} n$-Hexan gegeben. Das Reaktionsgemisch wird über Nacht auf Raumtemperatur gebracht und im Hochvakuum (ca. 0,01 mbar) vom entstandenen LiF abgetrennt. Die Verbindungen 33 und 34 erhält man als gelblichen Feststoff durch Sublimation in einem Kugelrohrofen. Umkristallisation in $n$-Hexan liefert für Verbindung 34 röntgentaugliche Einkristalle.

\section{Verbindung 35}

0,005 mol (1,7 g) 12 werden in $40 \mathrm{~mL} n$-Hexan gelöst und 0,005 mol $(0,2 \mathrm{~g})$ Methanol hinzugegeben. Verbindung 12 setzt sich quantitativ zu Verbindung 35 um. Das Lösungsmittel wird entfernt. Eine weitere Aufarbeitung ist nicht erforderlich.

Verbindung 35 entsteht ebenfalls durch Zugabe von Methanol zu Verbindung 11. 


\section{Verbindungen 36, 37, 38}

Für Verbindung

36 werden $0,08 \mathrm{~mol}(35,4 \mathrm{~g})$ Verbindung 29 in $200 \mathrm{~mL}$ THF gelöst, auf $-90{ }^{\circ} \mathrm{C}$ gekühlt und dann $0,08 \mathrm{~mol}(52,1 \mathrm{~mL})$ Methyllithium (5\%ig in Diethylether) zugetropft. Das Reaktionsgemisch wird über Nacht auf Raumtemperatur gebracht und das entstandene LiF durch Umkondensation im Vakuum abgetrennt.

37 werden $0,08 \mathrm{~mol}(35,4 \mathrm{~g})$ Verbindung 29 in $200 \mathrm{~mL} n$-Hexan gelöst und auf $-15^{\circ} \mathrm{C}$ gekühlt. Danach wird die Aufschlämmung mit $0,08 \mathrm{~mol}(34,1 \mathrm{~g})$ $n$-Butyllithium (15\%ig in $n$-Hexan) versetzt. Das Reaktionsgemisch wird über Nacht auf Raumtemperatur erwärmt und das Rohprodukt im Vakuum vom entstandenen LiF abgetrennt.

38 werden 0,08 mol (35,4 g) Verbindung 29 in $200 \mathrm{~mL}$ Diethylether gelöst und auf $-20{ }^{\circ} \mathrm{C}$ gekühlt. Anschließend gibt man 0,08 mol (40 mL) Phenyllithium (2,0 mol / L in Cyclohexan / Diethylether) hinzu. Die Lösung wird innerhalb von $4 \mathrm{~h}$ aufgetaut und $2 \mathrm{~d}$ auf Siedetemperatur gehalten. Das entstehende LiF wird abzentrifugiert.

Die Verbindungen 36, 37 und 38 werden durch fraktionierte Destillation (36 ca. 0,1 mbar; 37, 38 ca. 0,01 mbar) rein erhalten.

\section{Verbindung 39}

$0,05 \mathrm{~mol}(19,3 \mathrm{~g})$ von Verbindung 28 werden in $150 \mathrm{~mL} n$-Hexan gelöst und mit $0,05 \mathrm{~mol}$ (34 mL) t-Butyllithium (15\%ig in Pentan) versetzt. Nachdem die Lösung $3 \mathrm{~h}$ unter Rückfluss erhitzt wurde, ist die Lithiierung abgeschlossen. Zu der Suspension werden $50 \mathrm{~mL}$ THF hinzugegeben und auf $-60{ }^{\circ} \mathrm{C}$ gekühlt. Danach werden $0,05 \mathrm{~mol}$ $(5,4 \mathrm{~g}) \mathrm{Me}_{3} \mathrm{SiCl}$ langsam zugetropft. Das Reaktionsgemisch wird innerhalb von $4 \mathrm{~h}$ auf Raumtemperatur erwärmt und dann $4 \mathrm{~h}$ auf Siedetemperatur gehalten. Das Rohprodukt wird durch Umkondensation vom Lithiumfluorid abgetrennt, eingeengt und anschließend destilliert. Verbindung 39 kristallisierte in der Vorlage aus. 


\subsection{Behandlung und Entsorgung der Abfälle}

Die verwendeten Lösungsmittel werden destillativ entfernt oder in Kühlfallen einkondensiert. Falls keine Möglichkeit der Wiederverwertung besteht, werden sie getrennt nach halogenierten und nicht halogenierten Lösungsmittelabfällen in die dafür vorgesehenen Sammelbehälter gegeben und der zentralen Entsorgung zugeführt. Aceton, das zur Reinigung von Glasgeräten dient, wird destillativ zurückgewonnen.

Zum Trocknen von Lösungsmitteln verwendetes Natrium wird mit iso-Propanol oder Ethanol zersetzt und dem alkalischen Reinigungsbad zugeführt. Andere Trockenmittelreste wie $\mathrm{KOH}$ und $\mathrm{P}_{4} \mathrm{O}_{10}$ werden in die Basen- bzw. Säureabfälle überführt.

Säurebäder werden mit $\mathrm{Na}_{2} \mathrm{CO}_{3}$ neutralisiert und der zentralen Entsorgung zugeführt. Das zur Säuberung von Glasgeräten verwendete ethanolische Kaliumhydroxidbad wird durch Eindampfen reduziert und anschließend in die zentrale Sammelstelle gegeben. 


\section{Charakteristische Daten der dargestellten Verbindungen}

\subsection{Namen, Siede- und Schmelzpunkte, Ausbeuten sowie massen- und kernresonanzspektroskopische Daten}

Die Schmelzpunkte fester Stoffe wurden in einer Apparatur nach Tottoli in abgeschmolzenen Kapillaren bestimmt.

Die Molmassenbestimmung wurde massenspektroskopisch mit einem FINNIGAN MAT 8200- bzw. einem FINNIGAN MAT 9500-Gerät durchgeführt. Die Verbindungen wurden mit Hilfe der Elektronenstoßionisation (El; Elektronenanregungsenergie von $70 \mathrm{eV}$ ) ionisiert. Falls die Messungen einen Molpeak liefern, wird dieser als erstes angegeben. Danach folgen weitere charakteristische Fragmentpeaks in $\mathrm{m} / \mathrm{z}$. Angegeben ist zusätzlich die relative Intensität in bezug auf den Basispeak (100 \%) angegeben.

Alle dargestellten Verbindungen wurden kernresonanzspektroskopisch untersucht. Die chemischen Verschiebungen $\delta$ [ppm] und die Kopplungskonstanten $\mathrm{J}[\mathrm{Hz}] \mathrm{der}$ angefertigten ${ }^{1} \mathrm{H}-,{ }^{11} \quad \mathrm{~B}-,{ }^{13} \mathrm{C}-,{ }^{15} \mathrm{~N}$-, ${ }^{19} \mathrm{~F}$ - und ${ }^{29} \mathrm{Si}-N M R-S p e k t r e n$ sind auf den folgenden Seiten tabelliert. Die ${ }^{1} \mathrm{H}-,{ }^{11} \mathrm{~B}-,{ }^{13} \mathrm{C}-,{ }^{15} \mathrm{~N}$ - und ${ }^{29} \mathrm{Si}-K e r n e$ sind an einem BRUKER AVANCE 500- und BRUKER AVANCE 300-Kernresonanzspektrometer bei Raumtemperatur durchgeführt worden $\left({ }^{1} \mathrm{H},{ }^{13} \mathrm{C},{ }^{29} \mathrm{Si}\right.$ interner Standard TMS; ${ }^{11} \mathrm{~B}$ externer Standard $\mathrm{BF}_{3} \cdot \mathrm{OEt}_{2} ;{ }^{15} \mathrm{~N}$ externer Standard $\mathrm{MeNO}_{2}$ ). Die ${ }^{19} \mathrm{~F}-\mathrm{NMR}-$ Messungen erfolgten an einem BRUKER AVANCE 200-Kernresonanzspektrometer (interner Standard $\left.\mathrm{C}_{6} \mathrm{~F}_{6}\right)$. Die Messungen wurden aus $5 \%$ igen $\left({ }^{1} \mathrm{H},{ }^{19} \mathrm{~F}\right)$ bzw. die übrigen Kerne aus 30\%igen Lösungen gemacht.

Die Röntgenstrukturanalysen wurden DR. M. NOLTEMEYER und H.-G. SCHMIDT in der Abteilung von PROF. G.M. SHELDRICK am Institut für Anorganische Chemie der Universität Göttingen angefertigt. Die Datensammlungen wurden auf einem STOESIEMENS-AED-Vierkreisdiffraktometer bei graphitmonochromatischer $\mathrm{MoK}_{\alpha}$-Strahlung $(\lambda=71,069 \mathrm{pm})$ durchgeführt. Die Messung der Verbindungen erfolgte bei 
Tieftemperaturen im Bereich von $-80^{\circ} \mathrm{C}$ bis $-140^{\circ} \mathrm{C}$. Die Strukturen wurden durch "Direkte Methoden" gelöst und nach $\mathrm{F}^{2}$-Werten ("Kleinste-Quadrate-Verfahren") verfeinert. Für die Strukturlösung, -verfeinerung und -darstellung wurden die SHELXProgramme von PROF. G.M. SHELDRICK, Universität Göttingen, verwendet.

Ausgewählte Bindungslängen und -winkel werden in den jeweiligen Kapiteln angegeben. 
Verbindung 1: 2-(3,3-Dimethyl-1-(trimethylsilyl)butan-2-yliden)-1-(3,3dimethylbutan-2-yliden)hydrazin<smiles>CC(C[Si](C)(C)C)=NN=C(C)C(C)(C)C</smiles>

Summenformel: $\quad \mathrm{C}_{15} \mathrm{H}_{32} \mathrm{~N}_{2} \mathrm{Si}$

Molare Masse: $\quad 268,51 \mathrm{~g} / \mathrm{mol}$

MS (EI)

$268 \mathrm{~g} / \mathrm{mol}$

$12 \% \quad[\mathrm{M}]^{+}$

$253 \mathrm{~g} / \mathrm{mol}$

$36 \% \quad[\mathrm{M}-\mathrm{Me}]^{+}$

$211 \mathrm{~g} / \mathrm{mol}$

$33 \% \quad[\mathrm{M}-\mathrm{tBu}]^{+}$

$57 \mathrm{~g} / \mathrm{mol}$

$100 \% \quad[t \mathrm{Bu}]^{+}$

Siedepunkt: $\quad 74{ }^{\circ} \mathrm{C} / 0,7 \mathrm{mbar}$

Ausbeute: $\quad 83 \%$

NMR ( $\left.\mathrm{CDCl}_{3}\right)$ :

\begin{tabular}{|c|c|c|c|c|}
\hline \multirow[t]{4}{*}{$\delta{ }^{1} \mathrm{H}$ [ppm] } & 0,03 & $\mathrm{Si}\left(\mathrm{CH}_{3}\right)_{3}$ & s & $9 \mathrm{H}$ \\
\hline & 1,14 & $\mathrm{C}\left(\mathrm{C}_{3}\right)_{3}$ & $\mathrm{~s}$ & $18 \mathrm{H}$ \\
\hline & 1,78 & $\mathrm{C}_{\mathrm{H}_{3}}$ & $\mathrm{~s}$ & 0 \\
\hline & 1,91 & $\mathrm{CH}_{2}$ & $\mathrm{~s}$ & 2 \\
\hline \multirow[t]{9}{*}{$\delta^{13} \mathrm{C}[\mathrm{ppm}]$} & 0,5 & $\mathrm{Si}\left(\underline{\mathrm{CH}}_{3}\right)_{3}$ & s & \\
\hline & 13,0 & $\underline{\mathrm{CH}}_{3}$ & s & \\
\hline & 18,9 & $\underline{\mathrm{C}} \mathrm{H}_{2}$ & $\mathrm{~s}$ & \\
\hline & 27,9 & $\mathrm{C}\left(\underline{\mathrm{CH}}_{3}\right)_{3}$ & s & \\
\hline & 28,6 & $\mathrm{C}\left(\underline{\mathrm{CH}}_{3}\right)_{3}$ & s & \\
\hline & 38,3 & $\underline{\mathrm{C}}\left(\mathrm{CH}_{3}\right)_{3}$ & s & \\
\hline & 38,5 & $\underline{\mathrm{C}}\left(\mathrm{CH}_{3}\right)_{3}$ & s & \\
\hline & 167,4 & $\underline{\mathrm{CN}} \mathrm{a}$ & $\mathrm{s}$ & \\
\hline & 170,1 & $\underline{\mathrm{CN}}$ b & $s$ & \\
\hline
\end{tabular}


$\begin{array}{llll}\delta^{29} \mathrm{Si}[\mathrm{ppm}] & 0,9 & \underline{\mathrm{Si}}\left(\mathrm{CH}_{3}\right)_{3} \quad \mathrm{~s}\end{array}$ 
Verbindung 2: $\quad$ 2-(1-(Chlordimethylsilyl)-3,3-dimethylbutan-2-yliden)-1-(3,3dimethylbutan-2-yliden)hydrazin<smiles>C/C(=N/N=C(\C[Si](C)(C)Cl)C(C)(C)C)C(C)(C)C</smiles>

Summenformel: $\quad \mathrm{C}_{14} \mathrm{H}_{29} \mathrm{ClN}_{2} \mathrm{Si}$

Molare Masse: $\quad 288,93 \mathrm{~g} / \mathrm{mol}$

MS (EI)

$\begin{array}{lrl}288 \mathrm{~g} / \mathrm{mol} & 3 \% & {[\mathrm{M}]^{+}} \\ 273 \mathrm{~g} / \mathrm{mol} & 4 \% & {\left[_{\mathrm{M}-\mathrm{Me}]^{+}}\right.} \\ 253 \mathrm{~g} / \mathrm{mol} & 2 \% & {[\mathrm{M}-\mathrm{Cl}]^{+}} \\ 231 \mathrm{~g} / \mathrm{mol} & 8 \% & {[\mathrm{M}-\mathrm{Bu}]^{+}} \\ 57 \mathrm{~g} / \mathrm{mol} & 100 \% & {[t \mathrm{Bu}]^{+}}\end{array}$

Siedepunkt: $\quad 63^{\circ} \mathrm{C} / 1 \mathrm{mbar}$

Ausbeute: $\quad 12 \%$

$\operatorname{NMR}\left(\mathrm{CDCl}_{3}\right)$ :

\begin{tabular}{|c|c|c|c|}
\hline$\delta^{1} \mathrm{H}$ [ppm] & 0,47 & $\mathrm{Si}\left(\mathrm{C}_{3}\right)_{2}$ & $\mathrm{~s}$ \\
\hline & 1,15 & $\mathrm{C}\left(\mathrm{C}_{3}\right)_{3}$ & $\mathrm{~s}$ \\
\hline & 1,18 & $\mathrm{C}\left(\mathrm{C}_{3}\right)_{3}$ & $\mathrm{~s}$ \\
\hline & 1,82 & $\mathrm{C}_{\mathrm{H}_{3}}$ & $\mathrm{~s}$ \\
\hline & 2,25 & $\mathrm{CH}_{2}$ & $\mathrm{~s}$ \\
\hline$\delta^{13} \mathrm{C}[\mathrm{ppm}]$ & 3,5 & $\mathrm{Si}\left(\underline{\mathrm{C}}_{3}\right)_{2}$ & $s$ \\
\hline & 13,2 & $\underline{\mathrm{C}}_{3}$ & $\mathrm{~s}$ \\
\hline & 21,8 & $\underline{\mathrm{C}} \mathrm{H}_{2}$ & $\mathrm{~s}$ \\
\hline & 27,9 & $\mathrm{C}\left(\underline{\mathrm{C}}_{3}\right)_{3}$ & $\mathrm{~s}$ \\
\hline & 28,3 & $\mathrm{C}\left(\underline{\mathrm{C}}_{3}\right)_{3}$ & $\mathrm{~s}$ \\
\hline & 38,5 & $\underline{\mathrm{C}}\left(\mathrm{CH}_{3}\right)_{3}$ & $\mathrm{~s}$ \\
\hline & 38,7 & $\underline{\mathrm{C}}\left(\mathrm{CH}_{3}\right)_{3}$ & $\mathrm{~s}$ \\
\hline
\end{tabular}




$\begin{array}{llll}\delta{ }^{13} \mathrm{C}[\mathrm{ppm}] & 168,4 & \underline{\mathrm{CN}} & \mathrm{s} \\ 169,4 & \underline{\mathrm{CN}} & \mathrm{S}\end{array}$

$\delta^{29} \mathrm{Si}[\mathrm{ppm}] \quad 28,7 \quad \underline{\mathrm{SiCl}}\left(\mathrm{CH}_{3}\right)_{2} \quad \mathrm{~s}$ 
Verbindung 3: $\quad$ 2-(1-(Chlor(methyl)(phenyl)silyl)-3,3-dimethylbutan-2-yliden)1-(3,3-dimethylbutan-2-yliden)hydrazin

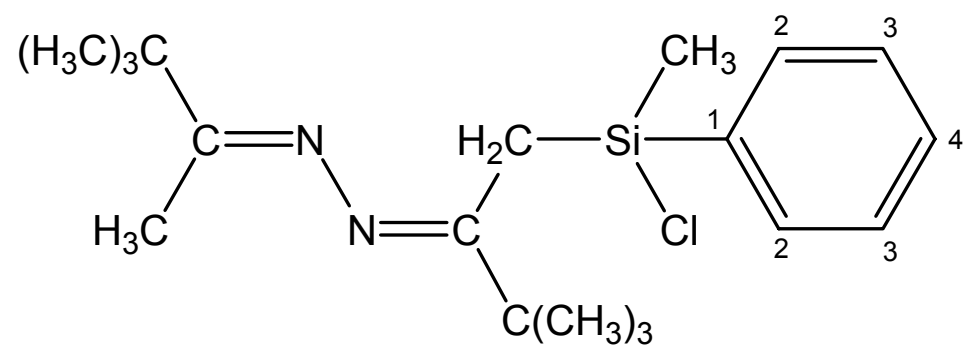

Summenformel: $\quad \mathrm{C}_{19} \mathrm{H}_{31} \mathrm{ClN}_{2} \mathrm{Si}$

Molare Masse: $\quad 351,00 \mathrm{~g} / \mathrm{mol}$

MS (EI)

$\begin{array}{lcl}350 \mathrm{~g} / \mathrm{mol} & 10 \% & {[\mathrm{M}]^{+}} \\ 335 \mathrm{~g} / \mathrm{mol} & 8 \% & {[\mathrm{M}-\mathrm{Me}]^{+}} \\ 293 \mathrm{~g} / \mathrm{mol} & 42 \% & {[\mathrm{M}-\mathrm{tBu}]^{+}} \\ 57 \mathrm{~g} / \mathrm{mol} & 100 \% & {[t \mathrm{Bu}]^{+}}\end{array}$

Siedepunkt: $\quad 108{ }^{\circ} \mathrm{C} / 0,009 \mathrm{mbar}$

Ausbeute: $\quad 68 \%$

$\operatorname{NMR}\left(\mathrm{CDCl}_{3}\right)$ :

$\delta{ }^{1} \mathrm{H}[\mathrm{ppm}]$

$\begin{array}{ll}0,73 & \mathrm{SiC}_{3} \\ 1,03 & \mathrm{C}\left(\mathrm{C}_{3}\right)_{3} \\ 1,17 & \mathrm{C}\left(\underline{\mathrm{H}}_{3}\right)_{3} \\ 1,81 & \mathrm{CH}_{3} \\ 2,50 & \underline{\mathrm{CH}}_{2} \mathrm{a} \\ 2,60 & \underline{\mathrm{C}}_{2} \mathrm{~b}\end{array}$

S

$3 \mathrm{H}$

s $9 \mathrm{H}$

s $9 \mathrm{H}$

s $3 \mathrm{H}$

d $\quad{ }^{2} J_{H_{a} H_{b}}=12,6 \mathrm{~Hz} \quad 1 \mathrm{H}$

7,3-7,7 $\quad \mathrm{C}_{6} \underline{\mathrm{H}}_{5}$

d $\quad{ }^{2} \mathrm{~J}_{\mathrm{H}_{b} \mathrm{H}_{\mathrm{a}}}=12,6 \mathrm{~Hz}$ $1 \mathrm{H}$

m

$\begin{array}{rrll}\delta{ }^{13} \mathrm{C}[\mathrm{ppm}] & 1,6 & \mathrm{Si} \underline{\mathrm{C}} \mathrm{H}_{3} & \mathrm{~s} \\ 13,3 & \underline{\mathrm{C}} \mathrm{H}_{3} & \mathrm{~s} \\ 20,7 & \underline{\mathrm{C}} \mathrm{H}_{2} & \mathrm{~s} \\ 27,7 & \mathrm{C}\left(\underline{\mathrm{C}} \mathrm{H}_{3}\right)_{3} & \mathrm{~s} \\ 28,5 & \mathrm{C}\left(\underline{\mathrm{C}} \mathrm{H}_{3}\right)_{3} & \mathrm{~s} \\ 38,5 & \underline{\mathrm{C}}\left(\mathrm{CH}_{3}\right)_{3} & \mathrm{~s}\end{array}$




$\begin{array}{rrlr}\delta{ }^{13} \mathrm{C}[\mathrm{ppm}] & 38,5 & \underline{\mathrm{C}}\left(\mathrm{CH}_{3}\right)_{3} & \mathrm{~s} \\ 128,0 & \mathrm{Ph}(\underline{\mathrm{C}} 3 / 2) & \mathrm{s} \\ 130,3 & \mathrm{Ph}(\underline{\mathrm{C}} 4) & \mathrm{s} \\ 133,3 & \mathrm{Ph}(\underline{\mathrm{C}} 2 / 3) & \mathrm{s} \\ 136,0 & \mathrm{Ph}(\underline{\mathrm{C}} 1) & \mathrm{s} \\ 167,7 & \underline{\mathrm{CN}} & \mathrm{s} \\ 169,3 & \underline{\mathrm{CN}} & \mathrm{s}\end{array}$

$\delta^{29} \mathrm{Si}[\mathrm{ppm}] \quad 16,4 \quad \mathrm{PhMe} \underline{\mathrm{SiCl}} \quad \mathrm{S}$ 
Verbindung 4: $\quad$ Bis(2-(2-(3,3-dimethylbutan-2-yliden)hydrazono)-3,3dimethylbut-1-yl)-dimethylsilan<smiles>CC(=N/N=C(/C)C[Si](C)(C)C/C(C)=N\N=C(C)C(C)(C)C)C(C)(C)C</smiles>

Summenformel: $\quad \mathrm{C}_{26} \mathrm{H}_{52} \mathrm{~N}_{4} \mathrm{Si}$

Molare Masse: $\quad 448,80 \mathrm{~g} / \mathrm{mol}$

MS (EI) $433 \mathrm{~g} / \mathrm{mol}$ $1 \% \quad[\mathrm{M}-\mathrm{Me}]^{+}$ $391 \mathrm{~g} / \mathrm{mol}$ $3 \% \quad[\mathrm{~m}-\mathrm{tBu}]^{+}$ $253 \mathrm{~g} / \mathrm{mol}$ $100 \% \quad[\mathrm{M}-\mathrm{R}]^{+}$

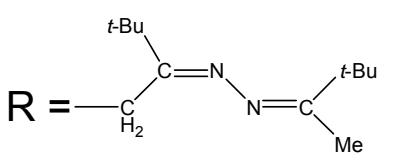

Siedepunkt: $\quad 130{ }^{\circ} \mathrm{C} / 0,008 \mathrm{mbar}$

Ausbeute: $\quad 29 \%$

NMR (CDCl 3 ):

$\begin{array}{rrlrr}\delta{ }^{1} \mathrm{H}[\mathrm{ppm}] & 0,06 & \mathrm{Si}\left(\mathrm{C}_{3}\right)_{2} & \mathrm{~s} & 6 \mathrm{H} \\ 1,14 & \mathrm{C}\left(\mathrm{C}_{\mathrm{H}_{3}}\right)_{3} & \mathrm{~s} & 18 \mathrm{H} \\ 1,15 & \mathrm{C}\left(\underline{\mathrm{C}}_{3}\right)_{3} & \mathrm{~s} & 18 \mathrm{H} \\ 1,80 & \mathrm{CH}_{3} & \mathrm{~s} & 6 \mathrm{H} \\ 2,04 & \mathrm{CH}_{2} & \mathrm{~s} & 4 \mathrm{H}\end{array}$

$\delta{ }^{13} \mathrm{C}[\mathrm{ppm}]$

$0,1 \quad \mathrm{Si}\left(\underline{\mathrm{CH}}_{3}\right)_{2}$

$\mathrm{S}$

$13,1 \quad \mathrm{CH}_{3}$

S

$20,7 \quad \underline{\mathrm{CH}_{2}}$

S

$28,0 \quad \mathrm{C}\left(\underline{\mathrm{CH}}_{3}\right)_{3}$

S

$28,6 \quad \mathrm{C}\left(\underline{\mathrm{CH}}_{3}\right)_{3}$ 


$\begin{array}{rrll}\delta{ }^{13} \mathrm{C}[\mathrm{ppm}] & 38,5 & \underline{\mathrm{C}}\left(\mathrm{CH}_{3}\right)_{3} & \mathrm{~s} \\ 38,6 & \underline{\mathrm{C}}\left(\mathrm{CH}_{3}\right)_{3} & \mathrm{~s} \\ 168,1 & \underline{\mathrm{CN}} & \mathrm{s} \\ 170,1 & \underline{\mathrm{CN}} & \mathrm{s}\end{array}$

$\begin{array}{llll}\delta^{29} \mathrm{Si}[\mathrm{ppm}] & 0,6 \quad \underline{\mathrm{Si}}\left(\mathrm{CH}_{3}\right)_{2} \quad \mathrm{~s}\end{array}$ 
Verbindung 5: $\quad$ Bis(2-(2-(3,3-dimethyl-1-(trimethylsilyl)butan-2-yliden)hydrazono)-3,3-dimethylbut-1-yl)-dimethylsilan

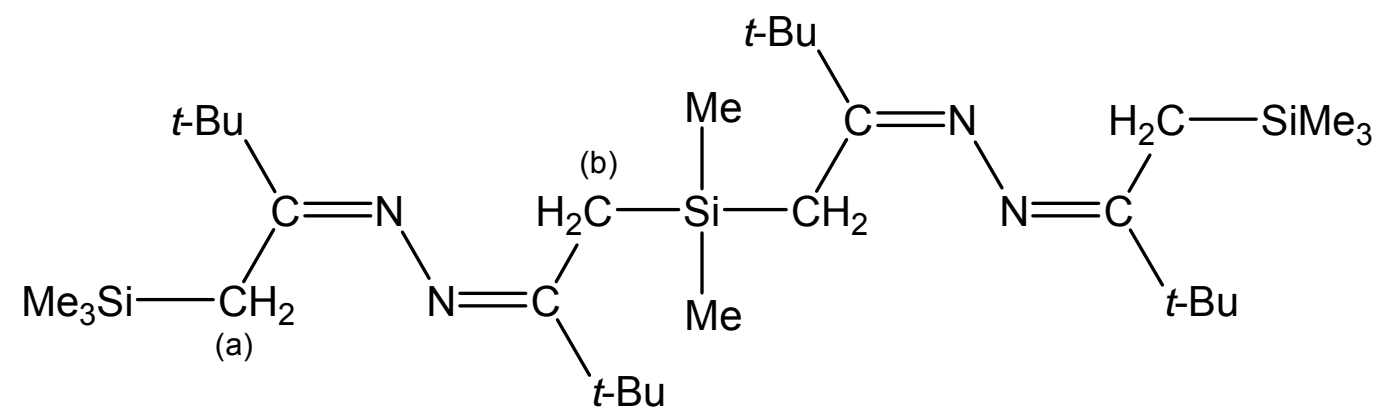

Summenformel: $\quad \mathrm{C}_{32} \mathrm{H}_{68} \mathrm{~N}_{4} \mathrm{Si}_{3}$

Molare Masse: $\quad 593,17 \mathrm{~g} / \mathrm{mol}$

MS (EI)

$577 \mathrm{~g} / \mathrm{mol}$

$1 \% \quad[\mathrm{M}-\mathrm{Me}]^{+}$

$325 \mathrm{~g} / \mathrm{mol}$

$100 \% \quad[\mathrm{M}-\mathrm{R}]^{+}$

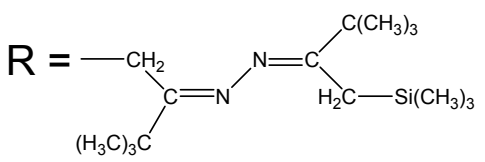

Siedepunkt: $\quad 173{ }^{\circ} \mathrm{C} / 0,07$ mbar

Ausbeute: $\quad 28 \%$

NMR (CDCl $)_{3}$ :

$\begin{array}{llllr}\delta{ }^{1} \mathrm{H} \text { [ppm] } & 0,05 & \mathrm{Si}\left(\mathrm{CH}_{3}\right)_{2} & \mathrm{~s} & 6 \mathrm{H} \\ & 0,05 & \mathrm{Si}\left(\mathrm{CH}_{3}\right)_{3} & \mathrm{~s} & 18 \mathrm{H} \\ 1,14 & \mathrm{C}\left(\mathrm{C}_{3}\right)_{3} & \mathrm{~s} & 18 \mathrm{H} \\ 1,15 & \mathrm{C}\left(\mathrm{C}_{3}\right)_{3} & \mathrm{~s} & 18 \mathrm{H} \\ 2,03 & \mathrm{CH}_{2} \mathrm{a} & \mathrm{s} & 4 \mathrm{H} \\ 2,16 & \mathrm{CH}_{2} \mathrm{~b} & \mathrm{~s} & 4 \mathrm{H}\end{array}$

$\begin{array}{rrll}\delta{ }^{13} \mathrm{C}[\mathrm{ppm}] & 0,7 & \mathrm{Si}\left(\underline{\mathrm{CH}}_{3}\right)_{2} & \mathrm{~s} \\ 1,1 & \mathrm{Si}\left(\underline{\mathrm{CH}}_{3}\right)_{3} & \mathrm{~s} \\ 20,2 & \underline{\mathrm{CH}}_{2} \mathrm{a} & \mathrm{s} \\ 22,1 & \underline{\mathrm{CH}}_{2} \mathrm{~b} & \mathrm{~s} \\ 29,3 & \mathrm{C}\left(\underline{\mathrm{C}} \mathrm{H}_{3}\right)_{3} & \mathrm{~s}\end{array}$




$\begin{array}{rrlr}\delta{ }^{13} \mathrm{C}[\mathrm{ppm}] & 29,4 & \left.\mathrm{C}(\underline{\mathrm{CH}})_{3}\right)_{3} & \mathrm{~s} \\ 38,9 & \underline{\mathrm{C}}\left(\mathrm{CH}_{3}\right)_{3} & \mathrm{~s} \\ 39,0 & \underline{\mathrm{C}}\left(\mathrm{CH}_{3}\right)_{3} & \mathrm{~s} \\ 172,8 & \underline{\mathrm{CN}} & \mathrm{s} \\ 173,3 & \underline{\mathrm{CN}} & \mathrm{s}\end{array}$

$\delta^{29} \mathrm{Si}[\mathrm{ppm}] \quad 0,7 \quad \underline{\mathrm{Si}}\left(\mathrm{CH}_{3}\right)_{2} \quad \mathrm{~s}$

$1,1 \quad \underline{\mathrm{Si}}\left(\mathrm{CH}_{3}\right)_{3} \quad \mathrm{~s}$ 
Verbindung 6: Tetra-[Lithium-[ $\mu^{3}$-(dimethyl-(3,5-di-tert-butyl-4,5-dihydro-5methyl-1H-pyrazol-1-yl)-silanolat)]]

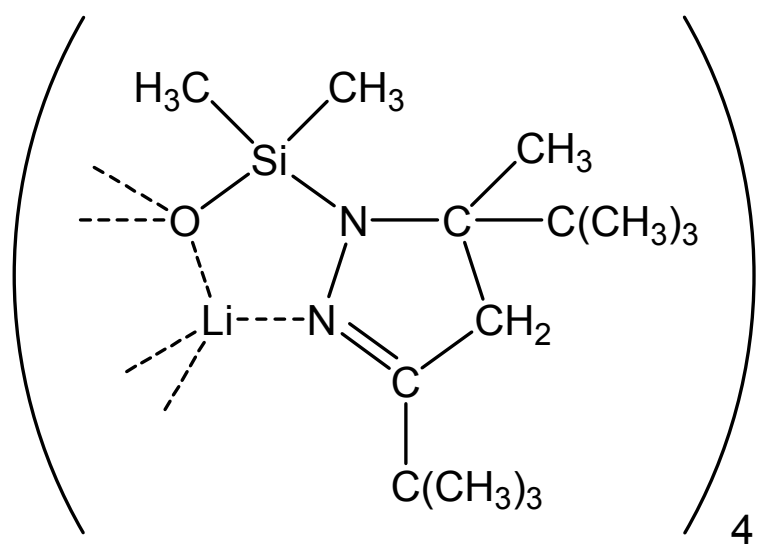

Summenformel: $\quad \mathrm{C}_{56} \mathrm{H}_{116} \mathrm{~N}_{8} \mathrm{Si}_{4}$

Molare Masse: $\quad 1105,68 \mathrm{~g} / \mathrm{mol}$

Schmelzpunkt: $\quad 108^{\circ} \mathrm{C}$

Ausbeute: $\quad 7 \%$

NMR $\left(\mathrm{CDCl}_{3}\right)$ :

$\delta{ }^{1} \mathrm{H}$ [ppm]

$\begin{array}{ll}0,40 & \mathrm{Si}\left(\mathrm{CH}_{3}\right)_{2} \\ 0,98 & \mathrm{C}\left(\mathrm{C}_{3}\right)_{3} \\ 1,29 & \mathrm{CH}_{3} \\ 1,32 & \mathrm{C}\left(\underline{\mathrm{CH}}_{3}\right)_{3} \\ 2,28 & \mathrm{C}_{2} \mathrm{a} \\ 2,78 & \mathrm{C}_{2} \mathrm{~b}\end{array}$

$2,78 \quad \mathrm{CH}_{2} \mathrm{~b}$

d

$$
{ }^{2} \mathrm{~J}_{\mathrm{H}_{\mathrm{a}} \mathrm{H}_{\mathrm{b}}}=17,5 \mathrm{~Hz}
$$
$\delta^{7} \mathrm{Li}$ [ppm]
$0,9 \underline{\operatorname{LiOSi}}\left(\mathrm{CH}_{3}\right)_{2}$
S 
Verbindung 7: 2-(1-(Dichlor(phenyl)silyl)-3,3-dimethylbutan-2-yliden)-1-(3,3dimethylbutan-2-yliden)hydrazin

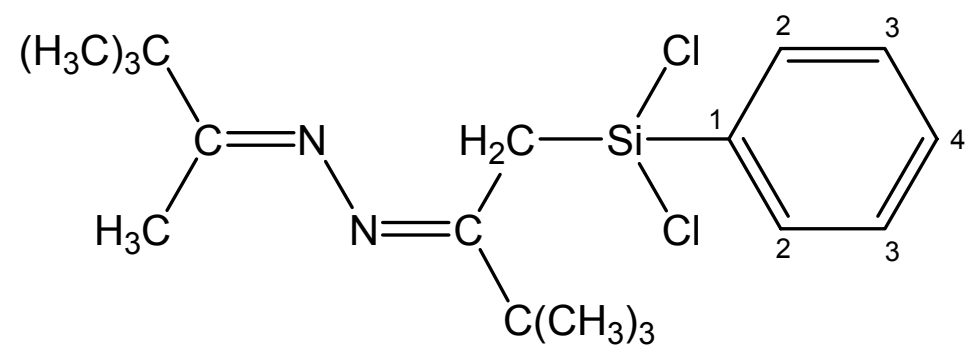

Summenformel: $\quad \mathrm{C}_{18} \mathrm{H}_{28} \mathrm{Cl}_{2} \mathrm{~N}_{2} \mathrm{Si}$

Molare Masse: $\quad 371,42 \mathrm{~g} / \mathrm{mol}$

MS (EI)

$\begin{array}{lrl}370 \mathrm{~g} / \mathrm{mol} & 8 \% & {[\mathrm{M}]^{+}} \\ 313 \mathrm{~g} / \mathrm{mol} & 18 \% & {[\mathrm{M}-\mathrm{tBu}]^{+}} \\ 57 \mathrm{~g} / \mathrm{mol} & 100 \% & {[\mathrm{tBu}]^{+}}\end{array}$

Siedepunkt: $\quad 108^{\circ} \mathrm{C} / 0,008 \mathrm{mbar}$

Ausbeute: $\quad 36 \%$

$\operatorname{NMR}\left(\mathrm{C}_{6} \mathrm{D}_{6}\right)$ :

$\delta^{1} \mathrm{H}[\mathrm{ppm}]$

$\begin{array}{rl}1,02 & \mathrm{C}\left(\mathrm{CH}_{3}\right)_{3} \\ 1,17 & \mathrm{C}\left(\underline{\mathrm{CH}}_{3}\right)_{3} \\ 1,85 & \mathrm{CH}_{3} \\ 2,97 & \mathrm{CH}_{2} \\ 7,1-7,8 & \mathrm{C}_{6} \underline{\mathrm{H}}_{5}\end{array}$

$\mathrm{S}$

S

S

S

$\mathrm{m}$

$\delta{ }^{13} \mathrm{C}[\mathrm{ppm}] \quad 13,8 \quad \underline{\mathrm{CH}}_{3} \quad \mathrm{~s}$

23,5 $\quad \underline{\mathrm{CH}}_{2}$

$27,8 \quad \mathrm{C}\left(\mathrm{CH}_{3}\right)_{3} \quad \mathrm{~s}$

28,5 $\mathrm{C}\left(\mathrm{CH}_{3}\right)_{3} \quad \mathrm{~s}$

$38,8 \quad \underline{\mathrm{C}}\left(\mathrm{CH}_{3}\right)_{3} \quad \mathrm{~s}$

$38,8 \quad \underline{\mathrm{C}}\left(\mathrm{CH}_{3}\right)_{3} \quad \mathrm{~s}$

128,4 Ph (ㄷ3/2) s

131,6 Ph (두) s

133,8 Ph $(\underline{\mathrm{C}} 2 / 3) \quad \mathrm{s}$
$9 \mathrm{H}$

$9 \mathrm{H}$

$3 \mathrm{H}$

$2 \mathrm{H}$

$5 \mathrm{H}$ 


$\begin{array}{rlll}\delta{ }^{13} \mathrm{C}[\mathrm{ppm}] & 133,8 & \mathrm{Ph}(\underline{\mathrm{C}} 1) & \mathrm{s} \\ 166,7 & \underline{\mathrm{CN}} & \mathrm{S} \\ 171,5 & \underline{\mathrm{CN}} & \mathrm{S}\end{array}$

$\delta^{29} \mathrm{Si}[\mathrm{ppm}] \quad 12,3 \quad \mathrm{PhSiCl}_{2} \quad \mathrm{~s}$ 
Verbindung 8: 2-(1-(Trichlorsilyl)-3,3-dimethylbutan-2-yliden)-1-(3,3dimethylbutan-2-yliden)hydrazin<smiles>CC(=NN=C(C)C(C)(C)C)C(C)(C)C</smiles>

Summenformel: $\quad \mathrm{C}_{12} \mathrm{H}_{23} \mathrm{Cl}_{3} \mathrm{~N}_{2} \mathrm{Si}$

Molare Masse: $\quad 329,77 \mathrm{~g} / \mathrm{mol}$

MS (EI)

$\begin{array}{lrl}330 \mathrm{~g} / \mathrm{mol} & 4 \% & {[\mathrm{M}]^{+}} \\ 315 \mathrm{~g} / \mathrm{mol} & 5 \% & {[\mathrm{M}-\mathrm{Me}]^{+}} \\ 273 \mathrm{~g} / \mathrm{mol} & 14 \% & {[\mathrm{M}-\mathrm{tBu}]^{+}} \\ 57 \mathrm{~g} / \mathrm{mol} & 100 \% & {[t \mathrm{Bu}]^{+}}\end{array}$

Siedepunkt: $\quad 98^{\circ} \mathrm{C} / 1,9 \mathrm{mbar}$

Ausbeute: $\quad 33 \%$

$\operatorname{NMR}\left(\mathrm{CDCl}_{3}\right)$ :

$\begin{array}{lllll}\delta{ }^{1} \mathbf{H}[\mathbf{p p m}] & 1,19 & \mathrm{C}\left(\mathrm{C}_{3}\right)_{3} & \mathrm{~s} & 9 \mathrm{H} \\ & 1,23 & \mathrm{C}\left(\mathrm{CH}_{3}\right)_{3} & \mathrm{~s} & 9 \mathrm{H} \\ 1,92 & \mathrm{CH}_{3} & \mathrm{~s} & 3 \mathrm{H} \\ 3,03 & \mathrm{CH}_{2} & \mathrm{~s} & 2 \mathrm{H}\end{array}$

$\begin{array}{rrlr}\delta{ }^{13} \mathrm{C} \text { [ppm] } & 13,9 & \underline{\mathrm{C}} \mathrm{H}_{3} & \mathrm{~s} \\ 27,0 & \underline{\mathrm{C}} \mathrm{H}_{2} & \mathrm{~s} \\ 27,7 & \mathrm{C}\left(\underline{\mathrm{C}} \mathrm{H}_{3}\right)_{3} & \mathrm{~s} \\ 28,1 & \mathrm{C}\left(\underline{\mathrm{C}} \mathrm{H}_{3}\right)_{3} & \mathrm{~s} \\ 38,6 & \underline{\mathrm{C}}\left(\mathrm{CH}_{3}\right)_{3} & \mathrm{~s} \\ 38,9 & \underline{\mathrm{C}}\left(\mathrm{CH}_{3}\right)_{3} & \mathrm{~s} \\ 165,2 & \underline{\mathrm{CN}} & \mathrm{s} \\ 172,5 & \underline{\mathrm{CN}} & \mathrm{s}\end{array}$


$\delta^{29} \mathrm{Si}[\mathrm{ppm}] \quad 5,5 \quad \underline{\mathrm{SiCl}}_{3} \quad \mathrm{~s}$ 
Verbindung 9: 2-(3,3-Dimethyl-1-(dimethyl(phenyl)silyl)butan-2-yliden)-1-

\section{(3,3-dimethylbutan-2-yliden)hydrazin}

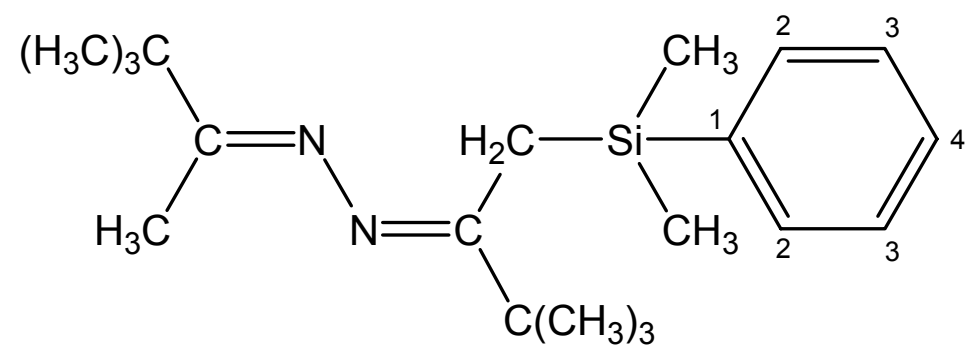

Summenformel: $\quad \mathrm{C}_{20} \mathrm{H}_{34} \mathrm{~N}_{2} \mathrm{Si}$

Molare Masse: $\quad 330,58 \mathrm{~g} / \mathrm{mol}$

MS (EI)

$\begin{array}{lll}330 \mathrm{~g} / \mathrm{mol} & 14 \% & {\left[_{\mathrm{M}]^{+}}\right.} \\ 315 \mathrm{~g} / \mathrm{mol} & 38 \% & {\left[_{\mathrm{M}-\mathrm{Me}]^{+}}\right.} \\ 273 \mathrm{~g} / \mathrm{mol} & 50 \% & {[\mathrm{M}-\mathrm{Bu}]^{+}} \\ 253 \mathrm{~g} / \mathrm{mol} & 14 \% & {[\mathrm{M}-\mathrm{Ph}]^{+}} \\ 135 \mathrm{~g} / \mathrm{mol} & 100 \% & {\left[\mathrm{SiMe}_{2} \mathrm{Ph}\right]^{+}}\end{array}$

Siedepunkt: $\quad 103{ }^{\circ} \mathrm{C} / 0,01 \mathrm{mbar}$

Ausbeute: $\quad 54 \%$

NMR $\left(\mathrm{CDCl}_{3}\right)$ :

\begin{tabular}{|c|c|c|c|}
\hline \multirow[t]{6}{*}{${ }^{1} \mathrm{H}$ rnnml } & 0,33 & $\mathrm{Si}\left(\mathrm{CH}_{3}\right)_{2}$ & $\mathrm{~s}$ \\
\hline & 1,07 & $\mathrm{C}\left(\mathrm{C}_{3}\right)_{3}$ & $\mathrm{~s}$ \\
\hline & 1,10 & $\mathrm{C}\left(\mathrm{C}_{3}\right)_{3}$ & $\mathrm{~s}$ \\
\hline & 1,79 & $\mathrm{CH}_{3}$ & $\mathrm{~s}$ \\
\hline & 2,19 & $\mathrm{CH}_{2}$ & $\mathrm{~s}$ \\
\hline & $7,3-7,5$ & $\mathrm{C}_{6} \underline{\mathrm{H}}_{5}$ & $\mathrm{~s}$ \\
\hline \multirow[t]{6}{*}{$\delta{ }^{13} \mathrm{C}[\mathrm{ppm}]$} & $-1,1$ & $\mathrm{Si}\left(\underline{\mathrm{CH}}_{3}\right)_{2}$ & $\mathrm{~s}$ \\
\hline & 13,0 & $\underline{\mathrm{CH}}_{3}$ & $\mathrm{~s}$ \\
\hline & 18,2 & $\underline{\mathrm{C}} \mathrm{H}_{2}$ & $\mathrm{~s}$ \\
\hline & 27,8 & $\mathrm{C}\left(\underline{\mathrm{C}}_{3}\right)_{3}$ & $\mathrm{~s}$ \\
\hline & 28,6 & $\mathrm{C}\left(\underline{\mathrm{CH}}_{3}\right)_{3}$ & $\mathrm{~s}$ \\
\hline & 38,3 & $\underline{\mathrm{C}}\left(\mathrm{CH}_{3}\right)_{3}$ & $\mathrm{~s}$ \\
\hline
\end{tabular}




$\begin{array}{rrll}\delta{ }^{13} \mathrm{C}[\mathrm{ppm}] & 38,5 & \underline{\mathrm{C}}\left(\mathrm{CH}_{3}\right)_{3} & \mathrm{~s} \\ 127,7 & \mathrm{Ph}(\underline{\mathrm{C}} 3 / 2) & \mathrm{s} \\ 128,9 & \mathrm{Ph}(\underline{\mathrm{C}} 4) & \mathrm{s} \\ 133,5 & \mathrm{Ph}(\underline{\mathrm{C}} 2 / 3) & \mathrm{s} \\ 139,9 & \mathrm{Ph}(\underline{\mathrm{C}} 1) & \mathrm{s} & \\ 167,6 & \underline{\mathrm{CN}} & \mathrm{s} & \\ 169,5 & \underline{\mathrm{CN}} & \mathrm{s} & { }^{2} \mathrm{~J}_{13 \mathrm{C} 29 \mathrm{Si}}=3,9 \mathrm{~Hz}\end{array}$

$\delta^{29} \mathrm{Si}[\mathrm{ppm}] \quad-4,6 \quad \underline{\mathrm{Si}}\left(\mathrm{CH}_{3}\right)_{2} \mathrm{Ph} \quad \mathrm{s}$ 
Verbindung 10: 1-(3,3-Dimethyl-1-(dimethyl(phenyl)silyl)butan-2-yliden)-2(3,3-dimethyl-1-(trimethylsilyl)butan-2-yliden)hydrazin<smiles>C/C(C[Si](C)(C)C)=N\N=C(\CC(C)(C)C)C[Si](C)(C)c1ccccc1</smiles>

Summenformel: $\quad \mathrm{C}_{23} \mathrm{H}_{42} \mathrm{~N}_{2} \mathrm{Si}_{2}$

Molare Masse: $\quad 402,76 \mathrm{~g} / \mathrm{mol}$

MS (EI)

$402 \mathrm{~g} / \mathrm{mol}$

$30 \% \quad[\mathrm{M}]^{+}$

$387 \mathrm{~g} / \mathrm{mol}$

$55 \% \quad[\mathrm{M}-\mathrm{Me}]^{+}$

$325 \mathrm{~g} / \mathrm{mol}$

$18 \% \quad[\mathrm{M}-\mathrm{Ph}]^{+}$

$135 \mathrm{~g} / \mathrm{mol}$

$100 \% \quad\left[\mathrm{SiMe}_{2} \mathrm{Ph}\right]^{+}$

Siedepunkt: $\quad 123^{\circ} \mathrm{C} / 0,02 \mathrm{mbar}$

Ausbeute:

$42 \%$

NMR ( $\left.\mathrm{CDCl}_{3}\right)$ :

$\delta^{1} \mathrm{H}$ [ppm]

$\begin{array}{rl}0,09 & \mathrm{Si}\left(\mathrm{CH}_{3}\right)_{3} \\ 0,35 & \mathrm{Si}\left(\mathrm{CH}_{3}\right)_{2} \\ 1,08 & \mathrm{C}\left(\mathrm{C}_{\mathrm{H}_{3}}\right)_{3} \\ 1,13 & \mathrm{C}\left(\mathrm{C}_{3}\right)_{3} \\ 2,06 & \mathrm{C}_{2} \\ 2,31 & \mathrm{PhSiC}_{2} \\ 7,3-7,5 & \mathrm{C}_{6} \underline{\mathrm{H}}_{5}\end{array}$

$\mathrm{S}$

$9 \mathrm{H}$

$\mathrm{S}$

$6 \mathrm{H}$

$\mathrm{s}$

$9 \mathrm{H}$

$S$

$9 \mathrm{H}$

S

$2 \mathrm{H}$

$\mathrm{S}$

$2 \mathrm{H}$

m

$5 \mathrm{H}$

$\delta{ }^{13} \mathrm{C}[\mathrm{ppm}]$

$\begin{array}{rlr}-1,0 & \mathrm{Si}\left(\underline{\mathrm{CH}}_{3}\right)_{2} & \mathrm{~s} \\ 0,7 & \mathrm{Si}\left(\underline{\mathrm{CH}}_{3}\right)_{3} & \mathrm{~s} \\ 18,8 & \mathrm{PhSi} \underline{\mathrm{C}} \mathrm{H}_{2} & \mathrm{~s} \\ 19,7 & \underline{\mathrm{CH}}_{2} & \mathrm{~s} \\ 28,8 & \mathrm{C}\left(\underline{\mathrm{CH}}_{3}\right)_{3} & \mathrm{~s} \\ 28,9 & \mathrm{C}\left(\underline{\mathrm{CH}}_{3}\right)_{3} & \mathrm{~s}\end{array}$

S

$\mathrm{S}$

$\mathrm{S}$

$S$

S

S 


$\begin{array}{rrlr}\delta{ }^{13} \mathrm{C} \text { [ppm] } & 38,4 & \underline{\mathrm{C}}\left(\mathrm{CH}_{3}\right)_{3} & \mathrm{~s} \\ 38,4 & \underline{\mathrm{C}}\left(\mathrm{CH}_{3}\right)_{3} & \mathrm{~s} \\ 127,7 & \mathrm{Ph}(\underline{\mathrm{C}} 3 / 2) & \mathrm{s} \\ 128,7 & \mathrm{Ph}(\underline{\mathrm{C}} 4) & \mathrm{s} \\ 133,5 & \mathrm{Ph}(\underline{\mathrm{C}} 2 / 3) & \mathrm{s} \\ 140,5 & \mathrm{Ph}(\underline{\mathrm{C}} 1) & \mathrm{s} \\ 171,9 & \underline{\mathrm{CN}} & \mathrm{s} \\ 172,8 & \underline{\mathrm{CN}} & \mathrm{s}\end{array}$

$\delta^{29} \mathrm{Si}[\mathrm{ppm}] \quad-4,4 \quad \mathrm{Ph} \underline{\mathrm{Si}}\left(\mathrm{CH}_{3}\right)_{2} \quad \mathrm{~s}$

$1,2 \quad \underline{\mathrm{Si}}\left(\mathrm{CH}_{3}\right)_{3} \quad \mathrm{~s}$ 
Verbindung 11: 3,3,5-Tri-tert-butyl-3,4-dihydro-2-(3,3-dimethylbut-1-en-2-yl)2H-1,2,3-diazasilol<smiles>C=C(N1N=C(C(C)(C)C)C[Si]1(C(C)(C)C)C(C)(C)C)C(C)(C)C</smiles>

Summenformel: $\quad \mathrm{C}_{20} \mathrm{H}_{40} \mathrm{~N}_{2} \mathrm{Si}$

Molare Masse: $\quad 336,63 \mathrm{~g} / \mathrm{mol}$

MS (EI)

$336 \mathrm{~g} / \mathrm{mol}$

$100 \% \quad[\mathrm{M}]^{+}$

$321 \mathrm{~g} / \mathrm{mol}$

$6 \% \quad[\mathrm{M}-\mathrm{Me}]^{+}$

Siedepunkt: $\quad 88^{\circ} \mathrm{C} / 0,008 \mathrm{mbar}$

Ausbeute:

$18 \%$

$\operatorname{NMR}\left(\mathrm{C}_{6} \mathrm{D}_{6}\right)$ :

$\delta{ }^{1} \mathrm{H}$ [ppm]

$\begin{array}{rl}1,07 & \mathrm{Si}\left(\mathrm{C}\left(\mathrm{CH}_{3}\right)_{3}\right)_{2} \\ 1,15 & \mathrm{C}\left(\mathrm{C}_{3}\right)_{3} \\ 1,46 & \mathrm{CH}_{2} \\ 1,51 & \mathrm{C}\left(\mathrm{C}_{3}\right)_{3} \\ 4,26 & =\mathrm{CH}_{2} \mathrm{a} \\ 4,27 & =\underline{\mathrm{C}}_{2} \mathrm{~b}\end{array}$

$\mathrm{s}$

$18 \mathrm{H}$

$\mathrm{s}$

$9 \mathrm{H}$

$\mathrm{S}$

$2 \mathrm{H}$

$\mathrm{s}$

$9 \mathrm{H}$

d $\quad{ }^{2} \mathrm{~J}_{\mathrm{H}_{\mathrm{a}} \mathrm{H}_{\mathrm{b}}}=0,8 \mathrm{~Hz}$

$1 \mathrm{H}$

d $\quad{ }^{2} \mathrm{~J}_{\mathrm{H}_{b} \mathrm{H}_{\mathrm{a}}}=0,8 \mathrm{~Hz}$

$1 \mathrm{H}$

$\begin{array}{clll}\delta{ }^{13} \mathrm{C} \text { [ppm] } & 13,0 & \underline{\mathrm{C}} \mathrm{H}_{2} & \mathrm{~s} \\ & 21,8 & \mathrm{Si}\left(\underline{\mathrm{C}}\left(\mathrm{CH}_{3}\right)_{3}\right)_{2} & \mathrm{~s} \\ 28,8 & \mathrm{C}\left(\underline{\mathrm{C}} \mathrm{H}_{3}\right)_{3} & \mathrm{~s} \\ 28,8 & \mathrm{Si}\left(\mathrm{C}\left(\underline{\mathrm{C}} \mathrm{H}_{3}\right)_{3}\right)_{2} & \mathrm{~s} \\ 30,5 & \mathrm{C}\left(\underline{\mathrm{C}} \mathrm{H}_{3}\right)_{3} & \mathrm{~s} \\ 36,7 & \underline{\mathrm{C}}\left(\mathrm{CH}_{3}\right)_{3} & \mathrm{~s} \\ 37,3 & \underline{\mathrm{C}}\left(\mathrm{CH}_{3}\right)_{3} & \mathrm{~s} \\ 85,6 & =\underline{\mathrm{C}} \mathrm{H}_{2} & \mathrm{~s}\end{array}$




$\begin{array}{lrlll}\delta{ }^{13} \mathrm{C}[\mathrm{ppm}] & 155,7 & \underline{\mathrm{CN}} & \mathrm{s} \\ & 161,1 & \underline{\mathrm{CN}} & \mathrm{s} & \\ & & & & \\ \delta{ }^{15} \mathrm{~N}[\mathrm{ppm}] & -232,0 & \underline{\mathrm{N}}-\mathrm{C} & \mathrm{d} & { }^{3} \mathrm{~J}_{\mathrm{NH}}=11,6 \mathrm{~Hz} \\ & -35,0 & \underline{\mathrm{C}}=\underline{\mathrm{N}} & \mathrm{d} & { }^{3} \mathrm{~J}_{\mathrm{NH}}=4,7 \mathrm{~Hz}\end{array}$

$\delta^{29} \mathrm{Si}[\mathrm{ppm}] \quad 27,6 \quad \underline{\mathrm{Si}}\left(\mathrm{C}\left(\mathrm{CH}_{3}\right)_{3}\right)_{2} \quad \mathrm{~s}$ 
Verbindung 12: $\quad N$-(2,2,4-Tri-tert-butyl-1,2-azasilet-1(2H)-yl)-3,3-dimethyl butan-2-imin

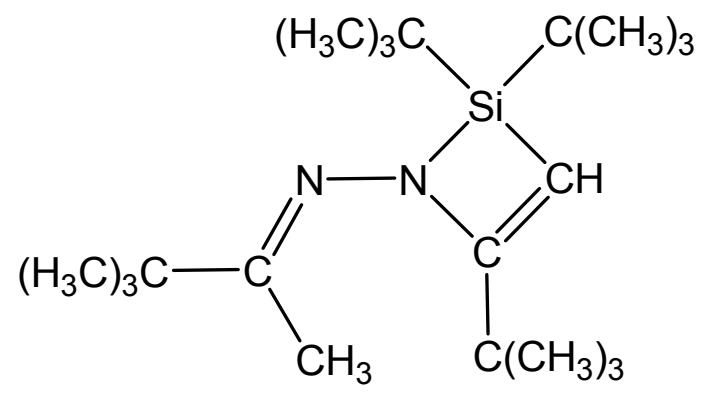

Summenformel: $\quad \mathrm{C}_{20} \mathrm{H}_{40} \mathrm{~N}_{2} \mathrm{Si}$

Molare Masse: $\quad 336,63 \mathrm{~g} / \mathrm{mol}$

MS (EI)

$336 \mathrm{~g} / \mathrm{mol}$

$100 \% \quad[\mathrm{M}]^{+}$

$321 \mathrm{~g} / \mathrm{mol}$

$7 \% \quad[\mathrm{M}-\mathrm{Me}]^{+}$

$279 \mathrm{~g} / \mathrm{mol}$

$18 \% \quad[\mathrm{M}-\mathrm{tBu}]^{+}$

Siedepunkt: $\quad 71^{\circ} \mathrm{C} / 0,02 \mathrm{mbar}$

Ausbeute: $\quad 15 \%$

$\operatorname{NMR}\left(\mathrm{C}_{6} \mathrm{D}_{6}\right)$ :

$\delta^{1} \mathrm{H}[\mathrm{ppm}]$

$\begin{array}{ll}1,11 & \mathrm{C}\left(\mathrm{C}_{3}\right)_{3} \\ 1,12 & \mathrm{Si}\left(\left(\mathrm{CH}_{3}\right)_{3}\right)_{2} \\ 1,36 & \mathrm{C}\left(\mathrm{C}_{3}\right)_{3} \\ 1,70 & \mathrm{CH}_{3} \\ 4,91 & =\underline{\mathrm{C}}\end{array}$

$S$

$9 \mathrm{H}$

S

$18 \mathrm{H}$

$\mathrm{S}$

$9 \mathrm{H}$

$\mathrm{s}$

$3 \mathrm{H}$

$\mathrm{s}$

${ }^{2} \mathrm{~J}_{1 \mathrm{H} 29 \mathrm{Si}}=12,2 \mathrm{~Hz}$

${ }^{3} \mathrm{~J}_{\mathrm{H}^{15 \mathrm{~N}}}=8,2 \mathrm{~Hz}$

$\begin{array}{llll}\delta{ }^{13} \mathrm{C} \text { [ppm] } & 12,5 & \underline{\mathrm{C}} \mathrm{H}_{3} & \mathrm{~s} \\ & 21,4 & \mathrm{Si}\left(\underline{\mathrm{C}}\left(\mathrm{CH}_{3}\right)_{3}\right)_{2} & \mathrm{~s} \\ 28,2 & \mathrm{Si}\left(\mathrm{C}\left(\underline{\mathrm{C}} \mathrm{H}_{3}\right)_{3}\right)_{2} & \mathrm{~s} \\ 28,4 & \mathrm{C}\left(\underline{\mathrm{C}} \mathrm{H}_{3}\right)_{3} & \mathrm{~s} \\ 28,6 & \mathrm{C}\left(\underline{\mathrm{C}} \mathrm{H}_{3}\right)_{3} & \mathrm{~s} \\ 37,1 & \underline{\mathrm{C}}\left(\mathrm{CH}_{3}\right)_{3} & \mathrm{~s} \\ 38,4 & \underline{\mathrm{C}}\left(\mathrm{CH}_{3}\right)_{3} & \mathrm{~s}\end{array}$




$\begin{array}{rrlll}\delta{ }^{13} \mathrm{C}[\mathrm{ppm}] & 92,0 & =\underline{\mathrm{CH}} & \mathrm{s} & { }^{1} \mathrm{~J}_{13 \mathrm{C}} \mathrm{C}^{29} \mathrm{Si}=62,3 \mathrm{~Hz} \\ 156,5 & \underline{\mathrm{CN}} & \mathrm{s} & \\ 182,2 & \underline{\mathrm{CN}} & \mathrm{s}\end{array}$

$\begin{array}{lllll}\delta{ }^{15} \mathrm{~N}[\mathrm{ppm}] & -232,5 & \underline{\mathrm{N}}-\mathrm{C} & \mathrm{d} & { }^{3} \mathrm{~J}_{\mathrm{NH}}=8,5 \mathrm{~Hz} \\ & -39,1 & \mathrm{C}=\underline{\mathrm{N}} & \mathrm{d} & { }^{4} \mathrm{~J}_{\mathrm{NH}}=3,7 \mathrm{~Hz}\end{array}$

$\delta{ }^{29} \mathrm{Si}[\mathrm{ppm}] \quad 33,3 \quad \underline{\mathrm{Si}}\left(\left(\mathrm{CH}_{3}\right)_{3}\right)_{2}$

s $\begin{aligned}{ }^{1} \mathrm{~J}_{29 \mathrm{Si}^{13} \mathrm{C}}=62,3 \mathrm{~Hz} \\ { }^{1} \mathrm{~J}_{29 \mathrm{Si}^{13} \mathrm{C}}=53,2 \mathrm{~Hz}\end{aligned}$


Verbindung 13: 2-(1-(tert-Butyl-(trimethylsilyl)amino)-difluorsilyl)-3,3dimethylbutan-2-yliden)-1-(3,3-dimethylbutan-2-yliden)hydrazin<smiles>CC(C[Si](F)(F)N([SiH3])C(C)(C)C)=NN=C(C)C(C)(C)C</smiles>

Summenformel: $\quad \mathrm{C}_{19} \mathrm{H}_{41} \mathrm{~F}_{2} \mathrm{~N}_{3} \mathrm{Si}_{2}$

Molare Masse: $\quad 405,72 \mathrm{~g} / \mathrm{mol}$ MS (EI)

$405 \mathrm{~g} / \mathrm{mol}$
$390 \mathrm{~g} / \mathrm{mol}$
$348 \mathrm{~g} / \mathrm{mol}$
$261 \mathrm{~g} / \mathrm{mol}$
$110{ }^{\circ} \mathrm{C} / 0,02 \mathrm{mbar}$
$40 \%$

$\begin{array}{cl}4 \% & {[\mathrm{M}]^{+}} \\ 6 \% & {[\mathrm{M}-\mathrm{Me}]^{+}} \\ 8 \% & {[\mathrm{M}-\mathrm{tBu}]^{+}} \\ 100 \% & {[\mathrm{M}-\mathrm{N} t \mathrm{BuSiMe}]^{+}}\end{array}$

Siedepunkt: $\quad 110{ }^{\circ} \mathrm{C} / 0,02 \mathrm{mbar}$

Ausbeute: $\quad 40 \%$

$\operatorname{NMR}\left(\mathrm{C}_{6} \mathrm{D}_{6}\right)$ :

\begin{tabular}{|c|c|c|c|c|}
\hline$\delta{ }^{1} \mathrm{H}[\mathrm{ppm}]$ & 0,24 & $\mathrm{NSi}\left(\mathrm{CH}_{3}\right)_{3}$ & $\mathrm{t}$ & ${ }^{5} \mathrm{~J}_{\mathrm{HF}}=1,1 \mathrm{~Hz}$ \\
\hline & 1,14 & $\mathrm{CC}\left(\mathrm{CH}_{3}\right)_{3}$ & $s$ & \\
\hline & 1,18 & $\mathrm{CC}\left(\mathrm{CH}_{3}\right)_{3}$ & $\mathrm{~s}$ & \\
\hline & 1,35 & $\mathrm{NC}\left(\mathrm{CH}_{3}\right)_{3}$ & $\mathrm{~s}$ & \\
\hline & 1,77 & $\mathrm{C}_{\mathrm{H}_{3}}$ & $s$ & \\
\hline & 2,23 & $\mathrm{C}_{\mathrm{H}_{2}}$ & $\mathrm{~m}$ & $A A^{\prime} X X^{\prime}$ \\
\hline$\delta{ }^{13} \mathrm{C}[\mathrm{ppm}]$ & 4,8 & $\mathrm{NSi}\left(\underline{\mathrm{CH}}_{3}\right)_{3}$ & $\mathrm{t}$ & ${ }^{4} \mathrm{~J}_{\mathrm{CF}}=2,4 \mathrm{~Hz}$ \\
\hline & 12,7 & $\underline{\mathrm{CH}}_{3}$ & $\mathrm{t}$ & ${ }^{7} \mathrm{~J}_{\mathrm{CF}}=1,2 \mathrm{~Hz}$ \\
\hline & 18,7 & $\underline{\mathrm{C}} \mathrm{H}_{2}$ & $\mathrm{t}$ & ${ }^{2} \mathrm{~J}_{\mathrm{CF}}=24,0 \mathrm{~Hz}$ \\
\hline & 27,1 & $\mathrm{CC}\left(\underline{\mathrm{CH}}_{3}\right)_{3}$ & $\mathrm{~s}$ & \\
\hline & 28,2 & $\mathrm{CC}\left(\underline{\mathrm{CH}}_{3}\right)_{3}$ & $\mathrm{~s}$ & \\
\hline & 33,3 & $\mathrm{NC}\left(\underline{\mathrm{CH}}_{3}\right)_{3}$ & $\mathrm{t}$ & ${ }^{4} \mathrm{~J}_{\mathrm{CF}}=2,3 \mathrm{~Hz}$ \\
\hline
\end{tabular}




\begin{tabular}{|c|c|c|c|c|}
\hline \multirow[t]{5}{*}{$\delta{ }^{13} \mathrm{C}[\mathrm{ppm}]$} & 38,0 & $\mathrm{C} \underline{\mathrm{C}}\left(\mathrm{CH}_{3}\right)_{3}$ & $\mathrm{~s}$ & \multirow[b]{3}{*}{${ }^{3} \mathrm{~J}_{\mathrm{CF}}=0,8 \mathrm{~Hz}$} \\
\hline & 38,1 & $\mathrm{C} \underline{\mathrm{C}}\left(\mathrm{CH}_{3}\right)_{3}$ & $\mathrm{~s}$ & \\
\hline & 53,9 & $\mathrm{~N} \underline{\mathrm{C}}\left(\mathrm{CH}_{3}\right)_{3}$ & $t$ & \\
\hline & 164,1 & $\underline{\mathrm{C}}=\mathrm{N}$ & $\mathrm{t}$ & \multirow[t]{2}{*}{${ }^{3} \mathrm{~J}_{\mathrm{CF}}=0,5 \mathrm{~Hz}$} \\
\hline & 168,0 & $\underline{\mathrm{C}}=\mathrm{N}$ & $\mathrm{s}$ & \\
\hline$\delta^{19} \mathrm{~F}[\mathrm{ppm}]$ & 45,2 & $\mathrm{SiF}_{2} \mathrm{~N}$ & $\mathrm{~m}$ & $A A^{\prime} X X^{\prime}$ \\
\hline \multirow[t]{2}{*}{$\delta{ }^{29} \mathrm{Si}$ [ppm] } & $-40,2$ & $\underline{\mathrm{SiF}}_{2} \mathrm{~N}$ & $\mathrm{t}$ & ${ }^{1} \mathrm{~J}_{\mathrm{SiF}}=283 \mathrm{~Hz}$ \\
\hline & 3,0 & $\mathrm{NSi}\left(\mathrm{CH}_{3}\right)_{3}$ & $\mathrm{t}$ & ${ }^{3} \mathrm{~J}_{\mathrm{SiF}}=1,3 \mathrm{~Hz}$ \\
\hline
\end{tabular}


Verbindung 14: Tris(2-(2-(3,3-dimethylbutan-2-yliden)hydrazono)-3,3dimethylbut-1-yl)-fluorsilan<smiles>CC(C)=N/N=C(/C)C[Si](F)(C/C(C)=N/N=C(C)C(C)(C)C)/C(C)=N/N=C(C)C(C)(C)C</smiles>

Summenformel: $\quad \mathrm{C}_{36} \mathrm{H}_{69} \mathrm{FN} \mathrm{N}_{6} \mathrm{Si}$

Molare Masse: $\quad 633,06 \mathrm{~g} / \mathrm{mol}$

MS (EI)

$555 \mathrm{~g} / \mathrm{mol}$

$10 \% \quad\left[\mathrm{M}-\mathrm{NC}(\mathrm{Me})\left(\mathrm{CMe}_{3}\right),-\mathrm{HF}\right]^{+}$

$437 \mathrm{~g} / \mathrm{mol}$ $100 \% \quad[M-R]^{+}$

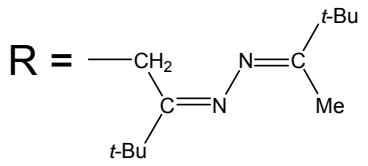

Siedepunkt: $\quad 124{ }^{\circ} \mathrm{C} / 0,04 \mathrm{mbar}$

Ausbeute: $\quad 5 \%$

$\operatorname{NMR}\left(\mathrm{CDCl}_{3}\right)$ :

$\delta^{1} \mathrm{H}[\mathrm{ppm}]$

$\begin{array}{ll}1,13 & \mathrm{C}\left(\mathrm{CH}_{3}\right)_{3} \\ 1,14 & \mathrm{C}\left(\mathrm{CH}_{3}\right)_{3} \\ 1,78 & \mathrm{C}_{3} \\ 2,22 & \underline{\mathrm{C}}_{2}\end{array}$

$S$

$27 \mathrm{H}$

d $\quad{ }^{6} \mathrm{~J}_{\mathrm{HF}}=0,4 \mathrm{~Hz}$

$27 \mathrm{H}$

$\mathrm{S}$

$9 \mathrm{H}$

$2,22 \quad \underline{\mathrm{CH}}_{2}$

d $\quad{ }^{3} \mathrm{~J}_{\mathrm{HF}}=7,8 \mathrm{~Hz}$

$6 \mathrm{H}$

$\delta{ }^{13} \mathrm{C}[\mathrm{ppm}]$

\section{3,3 $\quad \mathrm{CH}_{3}$}

S

$19,9 \quad \underline{\mathrm{CH}}_{2}$

d $\quad{ }^{2} \mathrm{~J}_{\mathrm{CF}}=13,7 \mathrm{~Hz}$

$28,0 \quad \mathrm{C}\left(\underline{\mathrm{CH}}_{3}\right)_{3}$

S 


$\begin{array}{rrlll}\delta{ }^{13} \mathrm{C} \text { [ppm] } & 28,8 & \mathrm{C}\left(\mathrm{CH}_{3}\right)_{3} & \mathrm{~d} & { }^{5} \mathrm{~J}_{\mathrm{CF}}=1,8 \mathrm{~Hz} \\ 38,7 & \underline{\mathrm{C}}\left(\mathrm{CH}_{3}\right)_{3} & \mathrm{~s} & \\ 38,9 & \underline{\mathrm{C}}\left(\mathrm{CH}_{3}\right)_{3} & \mathrm{~s} & \\ 167,6 & \underline{\mathrm{C}}=\mathrm{N} & \mathrm{d} & { }^{3} \mathrm{~J}_{\mathrm{CF}}=0,7 \mathrm{~Hz} \\ 169,1 & \underline{\mathrm{C}}=\mathrm{N} & \mathrm{s} & \end{array}$
$\delta{ }^{19} \mathrm{~F}[\mathrm{ppm}] \quad 19,4 \quad \mathrm{SiF}$
sept. $\quad{ }^{3} J_{F H}=7,8 \mathrm{~Hz}$
$\delta^{29} \mathrm{Si}[\mathrm{ppm}]$
$14,9 \quad \underline{\mathrm{SiF}}$
d $\quad{ }^{1} \mathrm{~J}_{\mathrm{SiF}}=308 \mathrm{~Hz}$ 
Verbindung 15: 5-tert-Butyl-3,4-dihydro-3,3-dimethyl-2-(3,3-dimethylbut-1-en2-yl)-2H-1,2,3-diazasilol<smiles>C=C(N1N=C(C(C)(C)C)C[Si]1(C)C)C(C)(C)C</smiles>

Summenformel: $\quad \mathrm{C}_{14} \mathrm{H}_{28} \mathrm{~N}_{2} \mathrm{Si}$

Molare Masse: $\quad 252,47 \mathrm{~g} / \mathrm{mol}$

MS (EI)

$252 \mathrm{~g} / \mathrm{mol}$

$97 \% \quad[\mathrm{M}]^{+}$

$237 \mathrm{~g} / \mathrm{mol}$

$64 \% \quad[\mathrm{M}-\mathrm{Me}]^{+}$

$195 \mathrm{~g} / \mathrm{mol}$

$38 \% \quad[\mathrm{M}-\mathrm{tBu}]^{+}$

Siedepunkt: $\quad 101^{\circ} \mathrm{C} / 7 \mathrm{mbar}$

Ausbeute: $\quad 59 \%$

$\operatorname{NMR}\left(\mathrm{CDCl}_{3}\right)$ :

$\delta{ }^{1} \mathrm{H}$ [ppm]

$0,32 \quad \mathrm{Si}\left(\mathrm{CH}_{3}\right)_{2}$

S

$6 \mathrm{H}$

$1,11 \quad \mathrm{C}\left(\mathrm{CH}_{3}\right)_{3}$

S

$9 \mathrm{H}$

$1,20 \quad \mathrm{C}\left(\mathrm{CH}_{3}\right)_{3}$

S

$9 \mathrm{H}$

$1,52 \quad \mathrm{CH}_{2}$

S

$2 \mathrm{H}$

$4,04=\mathrm{CH}_{2} \mathrm{a}$

$1 \mathrm{H}$

$4,06=\mathrm{CH}_{2} \mathrm{~b}$

$\mathrm{S}$

$1 \mathrm{H}$

$\begin{array}{cclc}\delta{ }^{13} \mathrm{C} \text { [ppm] } & -0,6 & \mathrm{Si}\left(\underline{\mathrm{C}} \mathrm{H}_{3}\right)_{2} & \mathrm{~s} \\ 16,2 & \underline{\mathrm{C}} \mathrm{H}_{2} & \mathrm{~s} \\ 28,6 & \mathrm{C}\left(\underline{\mathrm{C}} \mathrm{H}_{3}\right)_{3} & \mathrm{~s} \\ 29,8 & \mathrm{C}\left(\underline{\mathrm{C}} \mathrm{H}_{3}\right)_{3} & \mathrm{~s} \\ 36,5 & \underline{\mathrm{C}}\left(\mathrm{CH}_{3}\right)_{3} & \mathrm{~s} \\ 36,9 & \underline{\mathrm{C}}\left(\mathrm{CH}_{3}\right)_{3} & \mathrm{~s} \\ 87,0 & =\underline{\mathrm{C}} \mathrm{H}_{2} & \mathrm{~s}\end{array}$




$\begin{array}{llll}\delta{ }^{13} \mathrm{C}[\mathrm{ppm}] & 157,8 & \underline{\mathrm{CN}} & \mathrm{s} \\ 160,2 & \underline{\mathrm{CN}} & \mathrm{s}\end{array}$

$\delta^{29} \mathrm{Si}[\mathrm{ppm}] \quad 19,2 \quad \underline{\mathrm{Si}}\left(\mathrm{CH}_{3}\right)_{2} \quad$ s 
Verbindung 16: $\quad N$-(4-tert-Butyl-4-chlor-2,2-dimethyl-1,2-azasiletidin-1-yl)-3,3dimethylbutan-2-imin

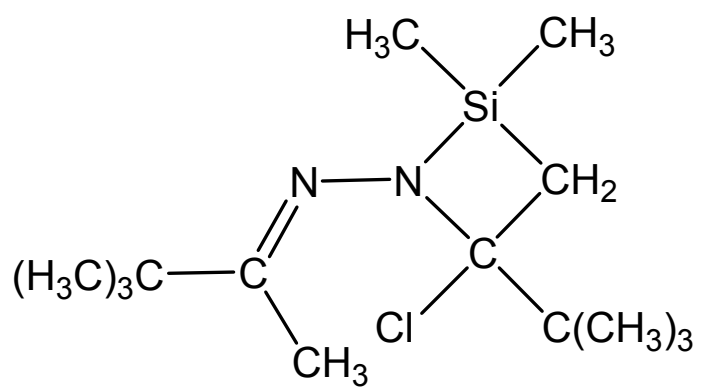

Summenformel: $\quad \mathrm{C}_{14} \mathrm{H}_{29} \mathrm{ClN}_{2} \mathrm{Si}$

Molare Masse: $\quad 288,93 \mathrm{~g} / \mathrm{mol}$

MS (EI)

$288 \mathrm{~g} / \mathrm{mol}$

$1 \% \quad[\mathrm{M}]^{+}$

$273 \mathrm{~g} / \mathrm{mol}$

$10 \% \quad[\mathrm{M}-\mathrm{Me}]^{+}$

$231 \mathrm{~g} / \mathrm{mol}$

$100 \% \quad[\mathrm{M}-\mathrm{tBu}]^{+}$

Siedepunkt: $\quad 105^{\circ} \mathrm{C} / 5 \mathrm{mbar}$

Ausbeute: $\quad 25 \%$

$\operatorname{NMR}\left(\mathrm{CDCl}_{3}\right)$ :

$\delta{ }^{1} \mathrm{H}$ [ppm]

$\begin{array}{ll}0,60 & \mathrm{Si}\left(\mathrm{CH}_{3}\right)_{2} \mathrm{a} \\ 0,62 & \mathrm{Si}\left(\mathrm{CH}_{3}\right)_{2} \mathrm{~b} \\ 0,84 & \mathrm{C}\left(\mathrm{CH}_{3}\right)_{3} \\ 1,10 & \mathrm{C}\left(\mathrm{C}_{3}\right)_{3} \\ 1,37 & \mathrm{CH}_{3} \\ 2,42 & \mathrm{CH}_{2} \mathrm{a} \\ 2,89 & \mathrm{C}_{2} \mathrm{~b}\end{array}$

$\mathrm{s}$

$3 \mathrm{H}$

(n)

$\left.\mathrm{Si}_{3}\right)_{2}$

S

$3 \mathrm{H}$

$\mathrm{s}$

$9 \mathrm{H}$

$\mathrm{s}$

$9 \mathrm{H}$

$\mathrm{S}$

$3 \mathrm{H}$

d $\quad{ }^{2} \mathrm{~J}_{\mathrm{H}_{\mathrm{a}} \mathrm{H}_{\mathrm{b}}}=17,3 \mathrm{~Hz}$

$1 \mathrm{H}$

d $\quad{ }^{2} \mathrm{~J}_{\mathrm{H}_{b} \mathrm{H}_{\mathrm{a}}}=17,3 \mathrm{~Hz}$

$1 \mathrm{H}$

$\begin{array}{rrll}\delta{ }^{13} \mathrm{C}[\mathrm{ppm}] & 4,2 & \mathrm{Si}\left(\mathrm{CH}_{3}\right)_{2} \mathrm{a} & \mathrm{s} \\ 4,9 & \mathrm{Si}\left(\underline{\mathrm{C}} \mathrm{H}_{3}\right)_{2} \mathrm{~b} & \mathrm{~s} \\ 23,4 & \underline{\mathrm{C}} \mathrm{H}_{3} & \mathrm{~s} \\ 25,4 & \mathrm{C}\left(\underline{\mathrm{C}} \mathrm{H}_{3}\right)_{3} & \mathrm{~s} \\ 28,3 & \mathrm{C}\left(\underline{\mathrm{CH}}_{3}\right)_{3} & \mathrm{~s} \\ 33,8 & \underline{\mathrm{C}}\left(\mathrm{CH}_{3}\right)_{3} & \mathrm{~s}\end{array}$




$\begin{array}{rrll}\delta{ }^{13} \mathrm{C}[\mathrm{ppm}] & 38,7 & \underline{\mathrm{C}}\left(\mathrm{CH}_{3}\right)_{3} & \mathrm{~s} \\ 44,4 & \underline{\mathrm{C}} \mathrm{H}_{2} & \mathrm{~s} \\ 72,2 & \underline{\mathrm{C}} \mathrm{Cl} & \mathrm{s} \\ 160,1 & \underline{\mathrm{C}}=\mathrm{N} & \mathrm{s}\end{array}$

$\begin{array}{llll}\delta^{29} \mathrm{Si}[\mathrm{ppm}] & 9,2 \quad \underline{\mathrm{Si}}\left(\mathrm{CH}_{3}\right)_{2} \quad \mathrm{~s}\end{array}$ 
Verbindung 17: 5-tert-Butyl-3,4-dihydro-3-methyl-2-(3,3-dimethylbut-1-en-2yl)-3-phenyl-2H-1,2,3-diazasilol

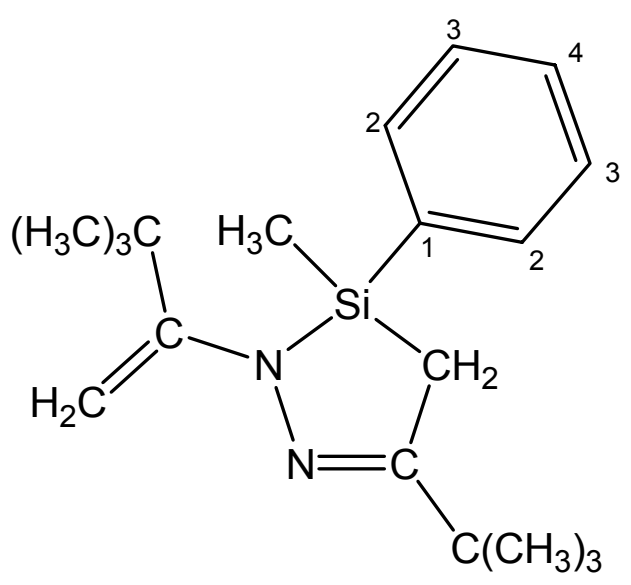

Summenformel: $\quad \mathrm{C}_{19} \mathrm{H}_{30} \mathrm{~N}_{2} \mathrm{Si}$

Molare Masse: $\quad 314,54 \mathrm{~g} / \mathrm{mol}$

MS (EI)

\begin{tabular}{|c|c|c|}
\hline $314 \mathrm{~g} / \mathrm{mol}$ & $100 \%$ & {$[\mathrm{M}]^{+}$} \\
\hline $299 \mathrm{~g} / \mathrm{mol}$ & $43 \%$ & {$[\mathrm{M}-\mathrm{Me}]^{+}$} \\
\hline $257 \mathrm{~g} / \mathrm{mol}$ & $77 \%$ & {$[\mathrm{M}-\mathrm{tBu}]^{+}$} \\
\hline
\end{tabular}

Siedepunkt: $\quad 79^{\circ} \mathrm{C} / 0,005 \mathrm{mbar}$

Ausbeute: $\quad 66 \%$

NMR $\left(\mathrm{CDCl}_{3}\right)$ :

$\delta{ }^{1} \mathrm{H}$ [ppm] $\quad 0,69 \quad \mathrm{SiC}_{3}$

$\begin{array}{llll}1,18 & \mathrm{C}\left(\mathrm{CH}_{3}\right)_{3} & \mathrm{~s} & 9 \mathrm{H}\end{array}$

$\begin{array}{llll}1,23 & \mathrm{C}\left(\mathrm{CH}_{3}\right)_{3} & \mathrm{~s} & 9 \mathrm{H}\end{array}$

$1,73 \mathrm{CH}_{2} \mathrm{a} \quad \mathrm{d} \quad{ }^{2} \mathrm{~J}_{\mathrm{H}_{\mathrm{a}} \mathrm{H}_{\mathrm{b}}}=19,1 \mathrm{~Hz} \quad 1 \mathrm{H}$

$1,79 \quad \mathrm{CH}_{2} \mathrm{~b} \quad \mathrm{~d} \quad{ }^{2} \mathrm{~J}_{\mathrm{H}_{\mathrm{b}} \mathrm{H}_{\mathrm{a}}=19,1 \mathrm{~Hz}} \quad 1 \mathrm{H}$

$3,89=\mathrm{CH}_{2}$ a $\quad$ d $\quad{ }^{2} \mathrm{~J}_{\mathrm{H}_{\mathrm{a}} \mathrm{H}_{\mathrm{b}}}=0,4 \mathrm{~Hz} \quad 1 \mathrm{H}$

$3,95=\underline{C}_{2} \mathrm{~b} \quad \mathrm{~d} \quad{ }^{2} \mathrm{~J}_{\mathrm{H}_{b} \mathrm{H}_{\mathrm{a}}}=0,4 \mathrm{~Hz} \quad 1 \mathrm{H}$

7,3-7,6 $\mathrm{C}_{6} \underline{\mathrm{H}}_{5} \quad \mathrm{~m} \quad 5 \mathrm{H}$

$\delta{ }^{13} \mathrm{C}$ [ppm] $\quad-4,1 \quad \mathrm{SiCH}_{3} \quad \mathrm{~S}$

$16,6 \quad \underline{\mathrm{CH}}_{2} \quad \mathrm{~s}$ 


$\begin{array}{rrlr}\delta{ }^{13} \mathrm{C}[\mathrm{ppm}] & 28,6 & \mathrm{C}\left(\underline{\mathrm{C}} \mathrm{H}_{3}\right)_{3} & \mathrm{~s} \\ 29,8 & \mathrm{C}\left(\underline{\mathrm{C}} \mathrm{H}_{3}\right)_{3} & \mathrm{~s} \\ 36,6 & \underline{\mathrm{C}}\left(\mathrm{CH}_{3}\right)_{3} & \mathrm{~s} \\ 36,9 & \underline{\mathrm{C}}\left(\mathrm{CH}_{3}\right)_{3} & \mathrm{~s} \\ 87,4 & =\underline{\mathrm{C}} \mathrm{H}_{2} & \mathrm{~s} \\ 128,0 & \mathrm{Ph}(\underline{\mathrm{C}} 3 / 2) & \mathrm{s} \\ 130,0 & \mathrm{Ph}(\underline{\mathrm{C}} 4) & \mathrm{s} \\ 133,8 & \mathrm{Ph}(\underline{\mathrm{C}} 2 / 3) & \mathrm{s} \\ 135,9 & \mathrm{Ph}(\underline{\mathrm{C}} 1) & \mathrm{s} \\ 157,6 & \underline{\mathrm{CN}} & \mathrm{s} \\ 159,7 & \underline{\mathrm{CN}} & \mathrm{s}\end{array}$

$\delta^{29} \mathrm{Si}[\mathrm{ppm}] \quad 9,1 \quad \underline{\mathrm{SiPhCH}}_{3} \quad \mathrm{~s}$ 
Verbindung 18: 5-tert-Butyl-3,4-dihydro-3-methyl-2-(3,3-dimethyl-1(trimethylsilyl)but-1-en-2-yl)-4-(trimethylsilyl)-3-phenyl-2H-

\section{1,2,3-diazasilol}

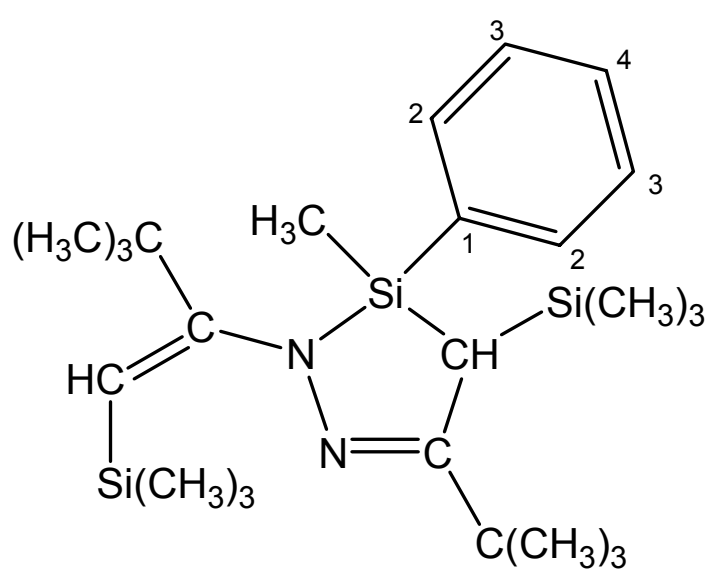

Summenformel: $\quad \mathrm{C}_{25} \mathrm{H}_{46} \mathrm{~N}_{2} \mathrm{Si}_{3}$

Molare Masse: $\quad 458,9 \mathrm{~g} / \mathrm{mol}$

MS (EI)

$458 \mathrm{~g} / \mathrm{mol}$
$443 \mathrm{~g} / \mathrm{mol}$
$401 \mathrm{~g} / \mathrm{mol}$
$73 \mathrm{~g} / \mathrm{mol}$

$\begin{aligned} 8 \% & {[\mathrm{M}]^{+} } \\ 9 \% & {[\mathrm{M}-\mathrm{Me}]^{+} } \\ 11 \% & {[\mathrm{M}-\mathrm{tBu}]^{+} } \\ 100 \% & \left.\text { [SiMe }_{3}\right]^{+}\end{aligned}$

Siedepunkt: $\quad 128{ }^{\circ} \mathrm{C} / 0,005$ mbar

Ausbeute: $42 \%$

Isomerengemisch im Verhältnis $A: B=4: 1$

$\operatorname{NMR}\left(\mathrm{CDCl}_{3}\right)$ :

Isomer A:

$\delta{ }^{1} \mathrm{H}[\mathrm{ppm}]$

$\begin{array}{rl}-0,03 & \mathrm{CSi}\left(\mathrm{CH}_{3}\right)_{3} \\ 0,22 & =\mathrm{CSi}\left(\mathrm{C}_{3}\right)_{3} \\ 0,80 & \mathrm{SiC} \underline{\mathrm{H}}_{3} \\ 1,24 & \mathrm{C}\left(\mathrm{C}_{3}\right)_{3} \\ 1,28 & \mathrm{C}\left(\mathrm{CH}_{3}\right)_{3} \\ 1,70 & \mathrm{C} \underline{\mathrm{H}} \\ 4,27 & =\mathrm{C} \underline{\mathrm{H}} \\ 7,3-7,6 & \mathrm{C}_{6} \underline{\mathrm{H}}_{5}\end{array}$

S

$9 \mathrm{H}$

$\mathrm{s}$

$9 \mathrm{H}$

S

$3 \mathrm{H}$

s

$9 \mathrm{H}$

S

$9 \mathrm{H}$

7,3-7,6 $\quad \mathrm{C}_{6} \underline{\mathrm{H}}_{5}$ 


$\begin{array}{rrlr}\delta{ }^{13} \mathrm{C}[\mathrm{ppm}] & -4,2 & \mathrm{Si} \underline{\mathrm{C}} \mathrm{H}_{3} & \mathrm{~s} \\ 2,2 & \mathrm{CSi}\left(\underline{\mathrm{C}} \mathrm{H}_{3}\right)_{3} & \mathrm{~s} \\ 2,6 & =\mathrm{CSi}\left(\underline{\mathrm{C}} \mathrm{H}_{3}\right)_{3} & \mathrm{~s} \\ 24,0 & \underline{\mathrm{C}} \mathrm{H} & \mathrm{s} \\ 30,0 & \mathrm{C}\left(\underline{\mathrm{C}} \mathrm{H}_{3}\right)_{3} & \mathrm{~s} \\ 30,9 & \mathrm{C}\left(\underline{\mathrm{CH}}_{3}\right)_{3} & \mathrm{~s} \\ 37,0 & \underline{\mathrm{C}}\left(\mathrm{CH}_{3}\right)_{3} & \mathrm{~s} \\ 38,3 & \underline{\mathrm{C}}(\mathrm{CH})_{3} & \mathrm{~s} \\ 102,2 & =\underline{\mathrm{C}} \mathrm{H} & \mathrm{s} \\ 128,0 & \mathrm{Ph}(\underline{\mathrm{C}} 3 / 2) & \mathrm{s} \\ 129,8 & \mathrm{Ph}(\underline{\mathrm{C}} 4) & \mathrm{s} \\ 133,3 & \mathrm{Ph}(\underline{\mathrm{C}} 2 / 3) & \mathrm{s} \\ 138,3 & \mathrm{Ph}(\underline{\mathrm{C}} 1) & \mathrm{s} \\ 158,3 & \underline{\mathrm{CN}} & \mathrm{s} \\ 166,3 & \underline{\mathrm{CN}} & \mathrm{s}\end{array}$

$\begin{array}{llll}\delta{ }^{29} \text { Si [ppm] } & -12,3 & =\underline{\mathrm{CSi}}\left(\mathrm{CH}_{3}\right)_{3} & \mathrm{~s} \\ -0,7 & \mathrm{CS}\left(\mathrm{CH}_{3}\right)_{3} & \mathrm{~s} \\ 14,5 & \underline{\mathrm{SiPhCH}}{ }_{3} & \mathrm{~s}\end{array}$


$\operatorname{NMR}\left(\mathrm{CDCl}_{3}\right)$ :

Isomer B:

$\delta{ }^{1} \mathrm{H}[\mathrm{ppm}]$

$\begin{array}{rlll}-0,16 & \mathrm{CSi}\left(\mathrm{CH}_{3}\right)_{3} & \mathrm{~s} & 9 \mathrm{H} \\ -0,09 & =\mathrm{CSi}\left(\mathrm{C}_{3}\right)_{3} & \mathrm{~s} & 9 \mathrm{H} \\ 0,60 & \mathrm{SiC}_{3} & \mathrm{~s} & 3 \mathrm{H} \\ 1,24 & \mathrm{C}\left(\mathrm{CH}_{3}\right)_{3} & \mathrm{~s} & 9 \mathrm{H} \\ 1,36 & \mathrm{C}\left(\mathrm{C}_{3}\right)_{3} & \mathrm{~s} & 9 \mathrm{H} \\ 1,44 & \mathrm{C} \underline{\mathrm{H}} & \mathrm{s} & 1 \mathrm{H} \\ 4,00 & =\mathrm{C} \underline{\mathrm{H}} & \mathrm{s} & 1 \mathrm{H} \\ 7,3-7,6 & \mathrm{C}_{6} \underline{\mathrm{H}}_{5} & \mathrm{~m} & 5 \mathrm{H}\end{array}$

$\delta{ }^{13} \mathrm{C}[\mathrm{ppm}] \quad-0,5 \quad \mathrm{SiCH}_{3} \quad \mathrm{~s}$

$1,9 \quad \mathrm{CSi}\left(\mathrm{CH}_{3}\right)_{3} \quad \mathrm{~S}$

$2,5 \quad=\mathrm{CSi}\left(\mathrm{CH}_{3}\right)_{3} \quad \mathrm{~s}$

$24,4 \quad \underline{\mathrm{C}} \mathrm{H} \quad \mathrm{S}$

$29,9 \quad \mathrm{C}\left(\mathrm{CH}_{3}\right)_{3} \quad \mathrm{~s}$

$30,8 \quad \mathrm{C}\left(\mathrm{CH}_{3}\right)_{3} \quad \mathrm{~s}$

$37,4 \quad \underline{\mathrm{C}}\left(\mathrm{CH}_{3}\right)_{3} \quad \mathrm{~s}$

$38,1 \quad \underline{\mathrm{C}}\left(\mathrm{CH}_{3}\right)_{3} \quad \mathrm{~s}$

$101,4=\underline{\mathrm{C}} \mathrm{H} \quad \mathrm{s}$

$127,6 \quad \mathrm{Ph}(\underline{\mathrm{C}} 3 / 2) \quad \mathrm{s}$

130,0 Ph (ㅁu) s

134,3 Ph $(\underline{\mathrm{C}} 1) \quad \mathrm{s}$

135,7 $\mathrm{Ph}(\underline{\mathrm{C}} 2 / 3) \quad \mathrm{s}$

$159,1 \quad \underline{\mathrm{C}} \quad \mathrm{s}$

$165,4 \quad \underline{\mathrm{CN}} \mathrm{s}$

$\delta^{29} \mathrm{Si}[\mathrm{ppm}] \quad-12,0 \quad=\underline{\mathrm{Ci}}\left(\mathrm{CH}_{3}\right)_{3} \quad \mathrm{~s}$

$-1,6 \quad \mathrm{C} \underline{\mathrm{Si}}\left(\mathrm{CH}_{3}\right)_{3} \quad \mathrm{~s}$

15,8 $\mathrm{SiPhCH}_{3} \quad \mathrm{~S}$ 
Verbindung 19: 5-tert-Butyl-3,3-dichlor-3,4-dihydro-2-(3,3-dimethylbut-1-en-2yl)-2H-1,2,3-diazasilol

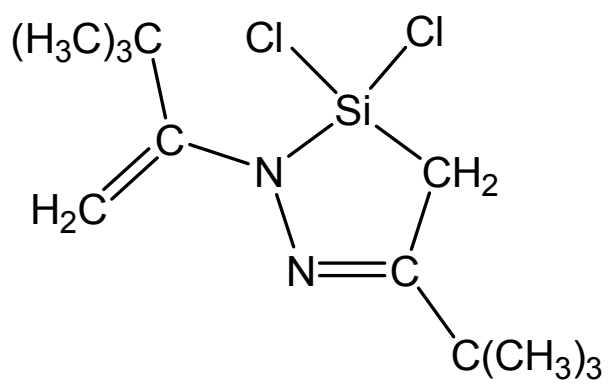

Summenformel: $\quad \mathrm{C}_{12} \mathrm{H}_{22} \mathrm{Cl}_{2} \mathrm{~N}_{2} \mathrm{Si}$

Molare Masse: $\quad 293,31 \mathrm{~g} / \mathrm{mol}$

MS (EI)

$$
\begin{aligned}
& 292 \mathrm{~g} / \mathrm{mol} \\
& 277 \mathrm{~g} / \mathrm{mol} \\
& 235 \mathrm{~g} / \mathrm{mol} \\
& 178 \mathrm{~g} / \mathrm{mol}
\end{aligned}
$$

$34 \% \quad[\mathrm{M}]^{+}$

$27 \% \quad[\mathrm{M}-\mathrm{Me}]^{+}$

$26 \% \quad[\mathrm{M}-\mathrm{tBu}]^{+}$

$100 \% \quad[\mathrm{M}-2 t \mathrm{Bu}]^{+}$

\begin{tabular}{|c|c|c|c|c|}
\hline$\delta^{1} \mathbf{H}$ [ppm] & 1,14 & $\mathrm{C}\left(\mathrm{C}_{3}\right)_{3}$ & $\mathrm{~s}$ & \\
\hline & 1,20 & $\mathrm{C}\left(\mathrm{C}_{3}\right)_{3}$ & s & \\
\hline & 2,02 & $\mathrm{C}_{\mathrm{H}_{2}}$ & $\mathrm{~s}$ & \\
\hline & 4,54 & $=\underline{C}_{2} a$ & $d$ & ${ }^{2} \mathrm{~J}_{\mathrm{H}_{\mathrm{a}} \mathrm{H}_{\mathrm{b}}}=0,8 \mathrm{~Hz}$ \\
\hline & 4,78 & $=\mathrm{C}_{2}{ }_{2} \mathrm{~b}$ & $d$ & ${ }^{2} J_{H_{b} H_{a}}=0,8 \mathrm{~Hz}$ \\
\hline$\delta{ }^{13} \mathrm{C}$ [ppm] & 18,8 & $\underline{\mathrm{CH}}_{2}$ & $\mathrm{~s}$ & \\
\hline & 28,2 & $\mathrm{C}\left(\underline{\mathrm{C}}_{3}\right)_{3}$ & s & \\
\hline & 29,6 & $\mathrm{C}\left(\underline{\mathrm{C}}_{3}\right)_{3}$ & s & \\
\hline & 36,9 & $\underline{\mathrm{C}}\left(\mathrm{CH}_{3}\right)_{3}$ & $\mathrm{~s}$ & \\
\hline & 37,1 & $\underline{\mathrm{C}}\left(\mathrm{CH}_{3}\right)_{3}$ & s & \\
\hline & 96,6 & $=\underline{\mathrm{C}} \mathrm{H}_{2}$ & s & \\
\hline & 156,4 & $\underline{\mathrm{CN}}$ & $s$ & \\
\hline
\end{tabular}

Siedepunkt: $\quad 121^{\circ} \mathrm{C} / 1 \mathrm{mbar}$

Ausbeute: $\quad 49 \%$

$\operatorname{NMR}\left(\mathrm{CDCl}_{3}\right)$ : 
$\delta{ }^{13} \mathrm{C}[\mathrm{ppm}] \quad 158,6 \quad \underline{\mathrm{CN}} \mathrm{s}$

$\delta{ }^{29} \mathrm{Si}[\mathrm{ppm}] \quad 2,0 \quad \underline{\mathrm{SiCl}}_{2} \quad \mathrm{~s}$ 
Verbindung 20: 5-tert-Butyl-3,3-dichlor-3,4-dihydro-2-(3,3-dimethyl-1(trimethylsilyl)but-1-en-2-yl)-4-(trimethylsilyl)-2H-1,2,3diazasilol<smiles>CC(C)(C)C1=NN(/C(=C\[SiH3])C(C)(C)C)[Si](Cl)(Cl)C1[SiH3]</smiles>

Summenformel: $\quad \mathrm{C}_{18} \mathrm{H}_{38} \mathrm{Cl}_{2} \mathrm{~N}_{2} \mathrm{Si}_{3}$

Molare Masse: $\quad 437,67 \mathrm{~g} / \mathrm{mol}$

MS (EI)

$436 \mathrm{~g} / \mathrm{mol}$
$421 \mathrm{~g} / \mathrm{mol}$
$379 \mathrm{~g} / \mathrm{mol}$
$57 \mathrm{~g} / \mathrm{mol}$

$\begin{array}{cl}6 \% & {[\mathrm{M}]^{+}} \\ 7 \% & {[\mathrm{M}-\mathrm{Me}]^{+}} \\ 8 \% & {[\mathrm{M}-t \mathrm{Bu}]^{+}} \\ 100 \% & {[\mathrm{BBu}]^{+}}\end{array}$

Siedepunkt: $\quad 117^{\circ} \mathrm{C} / 0,03 \mathrm{mbar}$

Ausbeute: $\quad 45 \%$

Isomerengemisch im

Verhältnis $A: B=2: 1$

$\operatorname{NMR}\left(\mathrm{CDCl}_{3}\right)$ :

Isomer A:

$\delta{ }^{1} \mathrm{H}[\mathrm{ppm}]$

$0,04 \quad \mathrm{CSi}\left(\mathrm{C}_{3}\right)_{3}$

$\mathrm{s}$

$9 \mathrm{H}$

$0,36=\mathrm{CSi}\left(\mathrm{C}_{\mathrm{H}_{3}}\right)_{3}$

$9 \mathrm{H}$

$1,14 \quad \mathrm{C}\left(\mathrm{C}_{3}\right)_{3}$

$9 \mathrm{H}$

$1,15 \quad \mathrm{C}\left(\mathrm{C}_{3}\right)_{3}$

$9 \mathrm{H}$

$1,82 \quad \mathrm{C} \underline{\mathrm{H}}$

$1 \mathrm{H}$

$5,51=\mathrm{C} \underline{\mathrm{H}}$

$\mathrm{S}$

$1 \mathrm{H}$
$\delta{ }^{13} \mathrm{C}[\mathrm{ppm}]$
0,5 $\mathrm{CSi}\left(\mathrm{CH}_{3}\right)_{3}$
$\mathrm{S}$
$2,1=\mathrm{CSi}\left(\underline{\mathrm{CH}}_{3}\right)_{3}$
$\mathrm{s}$
$27,5 \quad \underline{\mathrm{C}} \mathrm{H}$
S 


$\begin{array}{rrlr}\delta{ }^{13} \mathrm{C} \text { [ppm] } & 29,0 & \mathrm{C}\left(\underline{\mathrm{CH}}_{3}\right)_{3} & \mathrm{~s} \\ 30,8 & \mathrm{C}\left(\underline{\mathrm{C}} \mathrm{H}_{3}\right)_{3} & \mathrm{~s} \\ 37,8 & \underline{\mathrm{C}}\left(\mathrm{CH}_{3}\right)_{3} & \mathrm{~s} \\ 39,2 & \underline{\mathrm{C}}\left(\mathrm{CH}_{3}\right)_{3} & \mathrm{~s} \\ 122,8 & =\underline{\mathrm{C}} \mathrm{H} & \mathrm{s} \\ 158,2 & \underline{\mathrm{CN}} & \mathrm{s} \\ 162,2 & \underline{\mathrm{CN}} & \mathrm{s}\end{array}$

$\delta^{29} \mathrm{Si}[\mathrm{ppm}] \quad-9,0 \quad=\mathrm{C} \underline{\mathrm{Si}}\left(\mathrm{CH}_{3}\right)_{3} \quad \mathrm{~s}$

$-0,2 \quad \mathrm{CSi}\left(\mathrm{CH}_{3}\right)_{3} \quad \mathrm{~s}$

$1,6 \quad \mathrm{SiCl}_{2} \quad \mathrm{~s}$ 
$\operatorname{NMR}\left(\mathrm{CDCl}_{3}\right)$ :

Isomer B:

$\delta{ }^{1} \mathrm{H}[\mathrm{ppm}]$

$\begin{array}{ll}0,17 & \mathrm{CSi}\left(\mathrm{C}_{3}\right)_{3} \\ 0,30 & =\mathrm{CSi}\left(\underline{\mathrm{CH}}_{3}\right)_{3} \\ 1,18 & \mathrm{C}\left(\mathrm{C}_{3}\right)_{3} \\ 1,23 & \mathrm{C}\left(\mathrm{CH}_{3}\right)_{3} \\ 1,82 & \mathrm{C} \underline{\mathrm{H}} \\ 5,21 & =\underline{\mathrm{C}} \underline{\mathrm{H}}\end{array}$

S

S

S

$\mathrm{S}$

$\mathrm{S}$

S

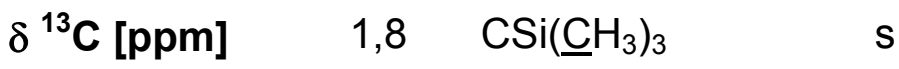

$2,3=\mathrm{CSi}\left(\mathrm{CH}_{3}\right)_{3} \quad \mathrm{~s}$

$27,6 \quad \underline{\mathrm{CH}} \quad \mathrm{S}$

$29,4 \quad \mathrm{C}\left(\mathrm{CH}_{3}\right)_{3} \quad \mathrm{~s}$

$30,7 \quad \mathrm{C}\left(\mathrm{CH}_{3}\right)_{3} \quad \mathrm{~s}$

$37,7 \quad \underline{\mathrm{C}}\left(\mathrm{CH}_{3}\right)_{3} \quad \mathrm{~s}$

$38,2 \quad \underline{\mathrm{C}}\left(\mathrm{CH}_{3}\right)_{3} \quad \mathrm{~s}$

$111,9=\underline{\mathrm{C}} \mathrm{H} \quad \mathrm{s}$

$159.1 \quad \underline{\mathrm{CN}} \mathrm{s}$

$162,1 \quad$ CN $\quad s$

$\delta^{29} \mathrm{Si}[\mathrm{ppm}] \quad-11,1 \quad=\mathrm{CSi}\left(\mathrm{CH}_{3}\right)_{3} \quad \mathrm{~s}$

$1,1 \quad \mathrm{C} \underline{\mathrm{Si}}\left(\mathrm{CH}_{3}\right)_{3} \quad \mathrm{~s}$

$3,0 \quad \mathrm{SiCl}_{2} \quad \mathrm{~s}$ 
Verbindung 21: 5-tert-Butyl-3,4-dihydro-3,3-diisopropyl-2-(3,3-dimethylbut-1en-2-yl)-2H-1,2,3-diazasilol<smiles>C=C(N1N=C(C(C)(C)C)C[Si]1(C(C)C)C(C)C)C(C)(C)C</smiles>

Summenformel: $\quad \mathrm{C}_{18} \mathrm{H}_{36} \mathrm{~N}_{2} \mathrm{Si}$

Molare Masse: $\quad 308,58 \mathrm{~g} / \mathrm{mol}$

MS (EI)

$\begin{array}{lrl}308 \mathrm{~g} / \mathrm{mol} & 70 \% & \text { [M] }^{+} \\ 293 \mathrm{~g} / \mathrm{mol} & 15 \% & {\text { [M-Me }]^{+}}_{2} \\ 265 \mathrm{~g} / \mathrm{mol} & 16 \% & {[\mathrm{M}-i \mathrm{Pr}]^{+}} \\ 209 \mathrm{~g} / \mathrm{mol} & 100 \% & {[\mathrm{M}-99 \mathrm{~g} / \mathrm{mol}]^{+}}\end{array}$

Siedepunkt: $\quad \quad \quad 70^{\circ} \mathrm{C} / 0,009 \mathrm{mbar}$

Ausbeute: $\quad 43 \%$

$\operatorname{NMR}\left(\mathrm{CDCl}_{3}\right)$ :

$\delta^{1} \mathrm{H}$ [ppm]

$\begin{array}{ll}0,98 & \mathrm{SiCH}\left(\mathrm{C}_{3}\right)_{2} \\ 1,00 & \mathrm{SiCH}\left(\mathrm{CH}_{3}\right)_{2} \\ 1,12 & \mathrm{C}\left(\mathrm{CH}_{3}\right)_{3} \\ 1,24 & \mathrm{C}\left(\underline{\mathrm{CH}}_{3}\right)_{3} \\ 1,31 & \mathrm{SiC} \underline{\mathrm{H}}\left(\mathrm{CH}_{3}\right)_{2} \\ 1,32 & \mathrm{SiC} \underline{\mathrm{H}}\left(\mathrm{CH}_{3}\right)_{2} \\ 1,45 & \mathrm{C} \underline{H}_{2} \\ 3,80 & =\mathrm{C}_{2} \mathrm{a} \\ 3,87 & =\mathrm{CH}_{2} \mathrm{~b}\end{array}$

d $\quad{ }^{3} \mathrm{~J}_{\mathrm{HH}}=7,4 \mathrm{~Hz}$

$6 \mathrm{H}$

d $\quad{ }^{3} \mathrm{~J}_{\mathrm{HH}}=7,4 \mathrm{~Hz}$

$6 \mathrm{H}$

$S$

$9 \mathrm{H}$

S

$9 \mathrm{H}$

sept $3^{3} \mathrm{~J}_{\mathrm{HH}}=7,4 \mathrm{~Hz} \quad 1 \mathrm{H}$

sept $\quad{ }^{3} \mathrm{~J}_{\mathrm{HH}}=7,4 \mathrm{~Hz} \quad 1 \mathrm{H}$

$2 \mathrm{H}$

d $\quad{ }^{2} \mathrm{~J}_{\mathrm{H}_{\mathrm{a}} \mathrm{H}_{\mathrm{b}}}=0,8 \mathrm{~Hz} \quad 1 \mathrm{H}$

d $\quad{ }^{2} \mathrm{~J}_{\mathrm{H}_{\mathrm{b}} \mathrm{H}_{\mathrm{a}}}=0,8 \mathrm{~Hz}$

$1 \mathrm{H}$
$\delta{ }^{13} \mathrm{C}$ [ppm]
9,1 $\underline{\mathrm{CH}}_{2}$
S
12,5 $\operatorname{SiC} H\left(\mathrm{CH}_{3}\right)_{2}$
$\mathrm{S}$ 


$\begin{array}{rrlr}\delta{ }^{13} \mathrm{C} \text { [ppm] } & 17,4 & \mathrm{SiCH}\left(\underline{\mathrm{C}} \mathrm{H}_{3}\right)_{2} & \mathrm{~s} \\ 17,8 & \mathrm{SiCH}\left(\underline{\mathrm{C}} \mathrm{H}_{3}\right)_{2} & \mathrm{~s} \\ 28,6 & \mathrm{C}\left(\underline{\mathrm{C}} \mathrm{H}_{3}\right)_{3} & \mathrm{~s} \\ 29,9 & \mathrm{C}\left(\underline{\mathrm{C}} \mathrm{H}_{3}\right)_{3} & \mathrm{~s} \\ 36,6 & \underline{\mathrm{C}}\left(\mathrm{CH}_{3}\right)_{3} & \mathrm{~s} \\ 36,8 & \underline{\mathrm{C}}\left(\mathrm{CH}_{3}\right)_{3} & \mathrm{~s} \\ 82,7 & =\underline{\mathrm{C}} \mathrm{H}_{2} & \mathrm{~s} \\ 155,9 & \underline{\mathrm{CN}} & \mathrm{s} \\ 161,1 & \underline{\mathrm{CN}} & \mathrm{s}\end{array}$

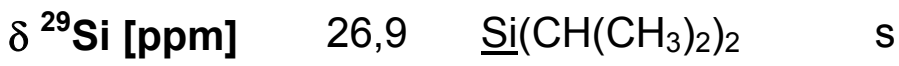


Verbindung 22: 1,2-Bis(1-(fluordiisopropylsilyl)-3,3-dimethylbutan-2yliden)hydrazin<smiles>CC(C[Si](F)(C(C)C)C(C)C)=NN=C(C)C[Si](F)(C(C)C)C(C)C</smiles>

Summenformel: $\quad \mathrm{C}_{24} \mathrm{H}_{50} \mathrm{~F}_{2} \mathrm{~N}_{2} \mathrm{Si}_{2}$

Molare Masse: $\quad 460,84 \mathrm{~g} / \mathrm{mol}$

MS (EI)

$460 \mathrm{~g} / \mathrm{mol}$

$58 \% \quad[\mathrm{M}]^{+}$

$445 \mathrm{~g} / \mathrm{mol}$

$9 \% \quad[\mathrm{M}-\mathrm{Me}]^{+}$

$417 \mathrm{~g} / \mathrm{mol}$

$86 \% \quad[\mathrm{M}-\mathrm{iPr}]^{+}$

$174 \mathrm{~g} / \mathrm{mol}$

$100 \% \quad[\mathrm{M} / 2-\mathrm{tBu}+\mathrm{H}]^{+}$

Siedepunkt: $\quad 82^{\circ} \mathrm{C} / 0,008 \mathrm{mbar}$

Ausbeute: $\quad 25 \%$

$\operatorname{NMR}\left(\mathrm{CDCl}_{3}\right)$ :

$\delta^{1} \mathrm{H}[\mathrm{ppm}]$

$\begin{array}{ll}1,00 & \mathrm{SiCH}\left(\mathrm{CH}_{3}\right)_{2} \text { a } \\ 1,03 & \mathrm{SiCH}\left(\mathrm{C}_{3}\right)_{2} \text { b } \\ 1,13 & \mathrm{SiCH}\left(\mathrm{CH}_{3}\right)_{2} \\ 1,18 & \mathrm{C}\left(\mathrm{CH}_{3}\right)_{3} \\ 2,21 & \mathrm{C}_{2}\end{array}$

d

$$
{ }^{3} \mathrm{~J}_{\mathrm{H}_{\mathrm{a}} \mathrm{H}_{\mathrm{b}}}=7,4 \mathrm{~Hz}
$$

$12 \mathrm{H}$

d

$$
3 \mathrm{~J}_{\mathrm{H}_{\mathrm{b}} \mathrm{H}_{\mathrm{a}}}=7,3 \mathrm{~Hz}
$$

$12 \mathrm{H}$

m

d $\quad{ }^{3} J_{H F}=9,2 \mathrm{~Hz}$

$\begin{array}{lrlll}\delta{ }^{13} \mathrm{C} \text { [ppm] } & 13,3 & \mathrm{SiC} H\left(\mathrm{CH}_{3}\right)_{2} & \mathrm{~d} & { }^{2} \mathrm{~J}_{\mathrm{CF}}=13,4 \mathrm{~Hz} \\ & 15,6 & \underline{\mathrm{CH}}_{2} & \mathrm{~d} & { }^{2} \mathrm{~J}_{\mathrm{CF}}=13,1 \mathrm{~Hz} \\ 17,0 & \mathrm{SiCH}\left(\underline{\mathrm{CH}}_{3}\right)_{2} \mathrm{a} & \mathrm{d} & { }^{3} \mathrm{~J}_{\mathrm{C}_{\mathrm{a}} \mathrm{F}}=1,9 \mathrm{~Hz} \\ 17,0 & \mathrm{SiCH}\left(\underline{\mathrm{CH}}_{3}\right)_{2} \mathrm{~b} & \mathrm{~d} & { }^{3} \mathrm{~J}_{\mathrm{C}_{\mathrm{b}} \mathrm{F}}=1,0 \mathrm{~Hz} \\ & 28,8 & \mathrm{C}\left(\underline{\mathrm{C}} \mathrm{H}_{3}\right)_{3} & \mathrm{~s} & \end{array}$




$\begin{array}{rrll}\delta{ }^{13} \mathrm{C}[\mathrm{ppm}] & 38,9 & \underline{\mathrm{C}}\left(\mathrm{CH}_{3}\right)_{3} & \mathrm{~s} \\ 173,1 & \underline{\mathrm{CN}} & \mathrm{s}\end{array}$
$\delta{ }^{19} \mathrm{~F}[\mathrm{ppm}] \quad-10,6 \quad \mathrm{SiF}$
m

$\delta^{29} \mathrm{Si}[\mathrm{ppm}] \quad 24,9 \quad \underline{\operatorname{SiF}}\left(\mathrm{CH}\left(\mathrm{CH}_{3}\right)_{2}\right)_{2} \quad \mathrm{~d} \quad{ }^{1} \mathrm{~J}_{\mathrm{SiF}}=303 \mathrm{~Hz}$ 
Verbindung 23: 3,5-Di-tert-butyl-3,4-dihydro-2-(3,3-dimethylbut-1-en-2-yl)-3phenyl-2H-1,2,3-diazasilol

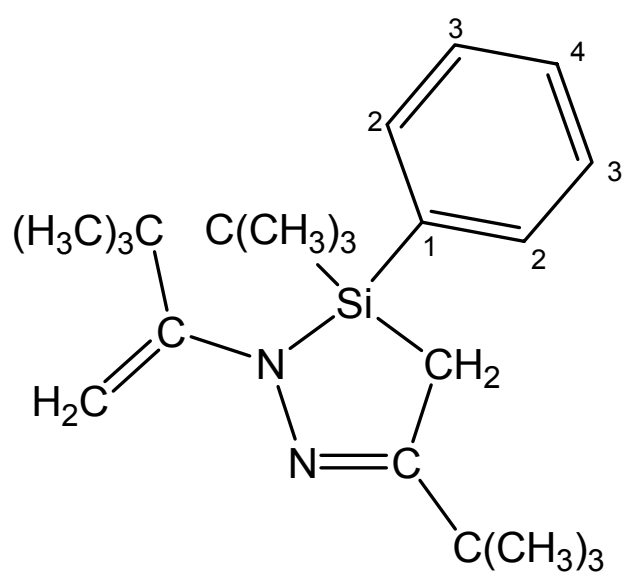

Summenformel: $\quad \mathrm{C}_{22} \mathrm{H}_{36} \mathrm{~N}_{2} \mathrm{Si}$

Molare Masse: $\quad 356,62 \mathrm{~g} / \mathrm{mol}$

MS (EI)

$356 \mathrm{~g} / \mathrm{mol}$

$34 \% \quad[\mathrm{M}]^{+}$

$341 \mathrm{~g} / \mathrm{mol}$

$8 \% \quad[\mathrm{M}-\mathrm{Me}]^{+}$

$279 \mathrm{~g} / \mathrm{mol}$

$12 \% \quad[\mathrm{M}-\mathrm{Ph}]^{+}$

$243 \mathrm{~g} / \mathrm{mol}$

$100 \% \quad\left[\mathrm{M}-\mathrm{tBu}-\mathrm{C}_{4} \mathrm{H}_{8}\right]^{+}$

Siedepunkt: $\quad 117^{\circ} \mathrm{C} / 0,05 \mathrm{mbar}$

Ausbeute: $\quad 61 \%$

NMR (CDCl $)_{3}$ :

$\delta^{1} \mathrm{H}[\mathrm{ppm}]$

$\begin{array}{rl}1,15 & \mathrm{CC}\left(\mathrm{C}_{3}\right)_{3} \\ 1,16 & \mathrm{SiC}\left(\mathrm{CH}_{3}\right)_{3} \\ 1,27 & \mathrm{CC}\left(\underline{\mathrm{CH}}_{3}\right)_{3} \\ 1,67 & \mathrm{C} \underline{\mathrm{H}}_{2} \mathrm{a} \\ 1,81 & \mathrm{C} \underline{\mathrm{H}}_{2} \mathrm{~b} \\ 3,98 & =\underline{\mathrm{C}}_{2} \mathrm{a} \\ 4,00 & =\underline{\mathrm{C}}_{2} \mathrm{~b} \\ 7,3-7,7 & \mathrm{C}_{6} \underline{\mathrm{H}}_{5}\end{array}$

S

$9 \mathrm{H}$

$\mathrm{S}$

$9 \mathrm{H}$

$\mathrm{S}$

$9 \mathrm{H}$

d

$1 \mathrm{H}$

d $\quad{ }^{2} \mathrm{~J}_{\mathrm{H}_{\mathrm{b}} \mathrm{H}_{\mathrm{a}}}=19,0 \mathrm{~Hz} \quad 1 \mathrm{H}$

d

${ }^{2} \mathrm{~J}_{\mathrm{H}_{\mathrm{a}} \mathrm{H}_{\mathrm{b}}}=0,7 \mathrm{~Hz}$

$1 \mathrm{H}$

d $\quad 2 \mathrm{~J}_{\mathrm{H}_{\mathrm{b}} \mathrm{H}_{\mathrm{a}}}=0,7 \mathrm{~Hz}$

$1 \mathrm{H}$

m

$5 \mathrm{H}$ 


$\begin{array}{rrlr}\delta{ }^{13} \mathrm{C}[\mathrm{ppm}] & 14,9 & \underline{\mathrm{C}} \mathrm{H}_{2} & \mathrm{~s} \\ 19,8 & \mathrm{SiC}\left(\mathrm{CH}_{3}\right)_{3} & \mathrm{~s} \\ 27.9 & \mathrm{SiC}\left(\underline{\mathrm{C}} \mathrm{H}_{3}\right)_{3} & \mathrm{~s} \\ 28,6 & \mathrm{CC}\left(\underline{\mathrm{C}} \mathrm{H}_{3}\right)_{3} & \mathrm{~s} \\ 30,1 & \mathrm{CC}\left(\underline{\mathrm{C}} \mathrm{H}_{3}\right)_{3} & \mathrm{~s} \\ 36,6 & \mathrm{C} \underline{\mathrm{C}}\left(\mathrm{CH}_{3}\right)_{3} & \mathrm{~s} \\ 37,1 & \mathrm{C} \underline{\mathrm{C}}\left(\mathrm{CH}_{3}\right)_{3} & \mathrm{~s} \\ 87,8 & =\underline{\mathrm{C}} \mathrm{H}_{2} & \mathrm{~s} \\ 128,0 & \mathrm{Ph}(\underline{\mathrm{C}} 3 / 2) & \mathrm{s} \\ 129,7 & \mathrm{Ph}(\underline{\mathrm{C}} 4) & \mathrm{s} \\ 134,6 & \mathrm{Ph}(\underline{\mathrm{C}} 2 / 3) & \mathrm{s} \\ 134,6 & \mathrm{Ph}(\underline{\mathrm{C}} 1) & \mathrm{s} \\ 156,7 & \underline{\mathrm{CN}} & \mathrm{s} \\ 160,5 & \underline{\mathrm{CN}} & \mathrm{s}\end{array}$

$\delta^{29} \mathrm{Si}[\mathrm{ppm}] \quad 12,7 \quad \underline{\operatorname{SiPh}}\left(\mathrm{CH}_{3}\right)_{3} \quad \mathrm{~s}$ 
Verbindung 24: 3,3,5,7-Tetra-tert-butyl-3,4-dihydro-2H-1,2,3-diazasilepin<smiles>CC(C)(C)C1=CC(C(C)(C)C)=NN[Si](C(C)(C)C)(C(C)(C)C)C1</smiles>

Summenformel: $\quad \mathrm{C}_{20} \mathrm{H}_{40} \mathrm{~N}_{2} \mathrm{Si}$

Molare Masse: $\quad 336,63 \mathrm{~g} / \mathrm{mol}$

MS (EI)

$336 \mathrm{~g} / \mathrm{mol}$

$12 \% \quad[\mathrm{M}]^{+}$

$321 \mathrm{~g} / \mathrm{mol}$

$3 \% \quad[\mathrm{M}-\mathrm{Me}]^{+}$

$279 \mathrm{~g} / \mathrm{mol}$

$33 \% \quad[\mathrm{M}-\mathrm{tBu}]^{+}$

$140 \mathrm{~g} / \mathrm{mol}$

$100 \% \quad[\mathrm{M}-196 \mathrm{~g} / \mathrm{mol}]^{+}$

Siedepunkt: $\quad 97^{\circ} \mathrm{C} / 0,008 \mathrm{mbar}$

Ausbeute: $\quad 13 \%$

$\operatorname{NMR}\left(\mathrm{CDCl}_{3}\right)$ :

$\delta^{1} \mathrm{H}$ [ppm]

$1,01 \quad \mathrm{Si}\left(\left(\mathrm{CH}_{3}\right)_{3}\right)_{2}$

S

$18 \mathrm{H}$

$1,11 \quad \mathrm{C}\left(\mathrm{CH}_{3}\right)_{3}$

$\mathrm{S}$

$9 \mathrm{H}$

$1,22 \quad \mathrm{C}\left(\mathrm{CH}_{3}\right)_{3}$

$\mathrm{S}$

$9 \mathrm{H}$

$1,60 \quad \mathrm{CH}_{2}$

$\mathrm{S}$

$2 \mathrm{H}$

$4,88 \quad \mathrm{~N} \underline{\mathrm{H}}$

$1 \mathrm{H}$

$5,83=\mathrm{C} \underline{\mathrm{H}}$

S

$1 \mathrm{H}$

$\begin{array}{cclll}\delta{ }^{13} \mathrm{C}[\mathrm{ppm}] & 10,6 & \underline{\mathrm{C}} \mathrm{H}_{2} & \mathrm{~s} & { }^{1} \mathrm{~J}_{13 \mathrm{C}}{ }^{13 \mathrm{C}}=38,6 \mathrm{~Hz} \\ & & & { }^{1} \mathrm{~J}_{13 \mathrm{C}^{29} \mathrm{Si}}=52,1 \mathrm{~Hz} \\ & 22,1 & \mathrm{Si}\left(\underline{\mathrm{C}}\left(\mathrm{CH}_{3}\right)_{3}\right)_{2} & \mathrm{~s} & { }^{1} \mathrm{~J}_{13 \mathrm{C} C}{ }^{29} \mathrm{Si}=55,6 \mathrm{~Hz} \\ 29,1 & \mathrm{Si}\left(\mathrm{C}\left(\underline{\mathrm{CH}}_{3}\right)_{3}\right)_{2} & \mathrm{~s} & \\ 29,3 & \mathrm{C}\left(\underline{\mathrm{C}} \mathrm{H}_{3}\right)_{3} & \mathrm{~s} & \\ 30,5 & \mathrm{C}\left(\underline{\mathrm{C}} \mathrm{H}_{3}\right)_{3} & \mathrm{~s} & { }^{1} \mathrm{~J}_{13 \mathrm{C}}{ }^{13 \mathrm{C}}=35,6 \mathrm{~Hz}\end{array}$




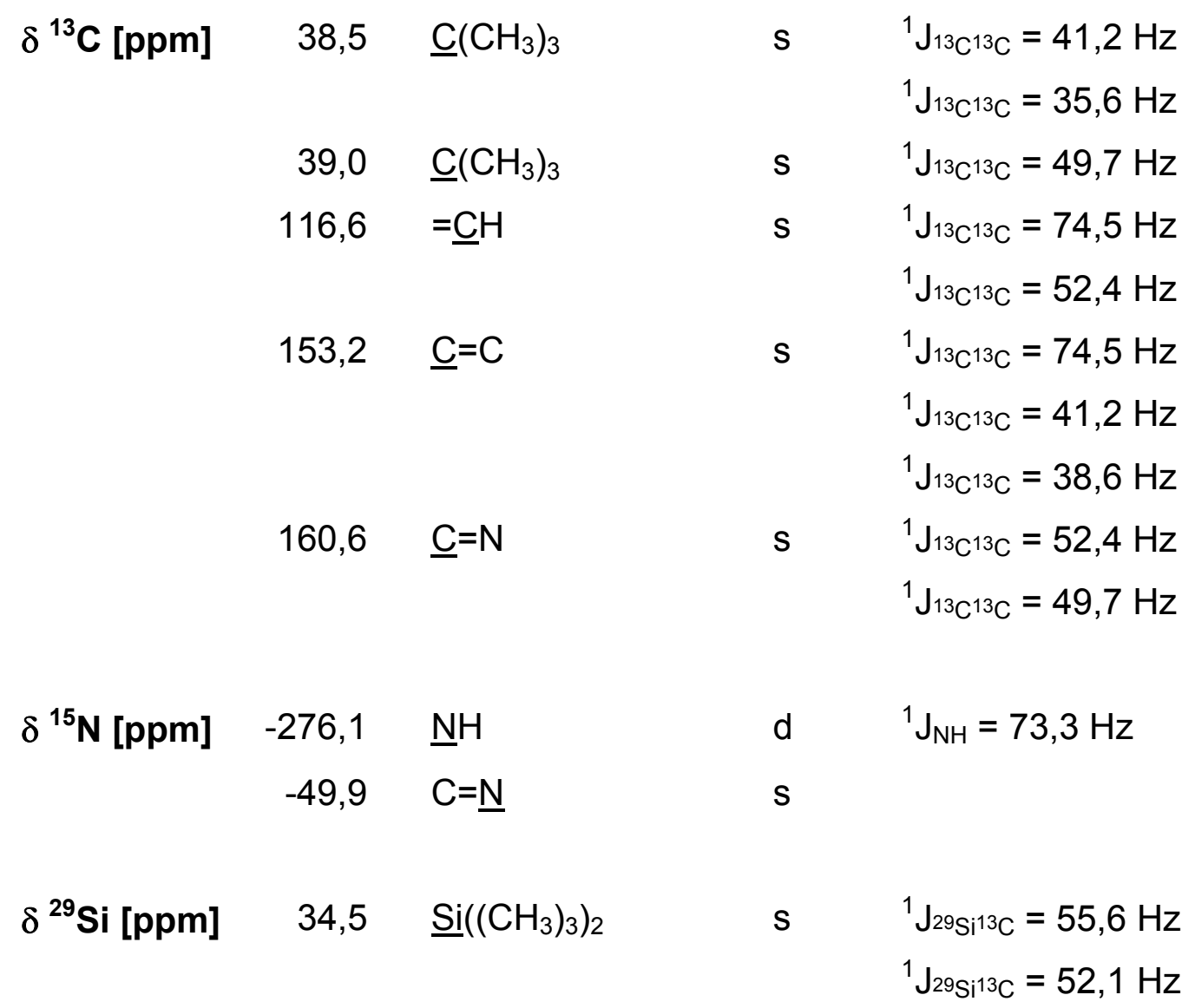


Verbindung 25: 3,3,5-Tri-tert-butyl-3,4-dihydro-2-(3,3-dimethyl-1(trimethylsilyl)but-1-en-2-yl)-2H-1,2,3-diazasilol<smiles>CC(C)(C)C1=NN(/C(=C\[SiH3])C(C)(C)C)[Si](C(C)(C)C)(C(C)(C)C)C1</smiles>

Summenformel: $\quad \mathrm{C}_{23} \mathrm{H}_{48} \mathrm{~N}_{2} \mathrm{Si}_{2}$

Molare Masse: $\quad 408,81 \mathrm{~g} / \mathrm{mol}$ MS (EI)

$408 \mathrm{~g} / \mathrm{mol}$
$393 \mathrm{~g} / \mathrm{mol}$
$351 \mathrm{~g} / \mathrm{mol}$
$295 \mathrm{~g} / \mathrm{mol}$

$14 \% \quad[\mathrm{M}]^{+}$

$13 \% \quad[\mathrm{M}-\mathrm{Me}]^{+}$

$12 \% \quad[\mathrm{M}-\mathrm{tBu}]^{+}$

$100 \% \quad\left[\mathrm{M}-\mathrm{tBu}-\mathrm{C}_{4} \mathrm{H}_{8}\right]^{+}$

Siedepunkt: $\quad 130{ }^{\circ} \mathrm{C} / 0,5 \mathrm{mbar}$

Ausbeute: $\quad 80 \%$

NMR $\left(\mathrm{CDCl}_{3}\right)$ :

\begin{tabular}{|c|c|c|c|c|}
\hline \multirow[t]{6}{*}{$\delta^{1} \mathrm{H}[\mathrm{ppm}]$} & 0,35 & $=\mathrm{CSi}\left(\mathrm{C}_{3}\right)_{3}$ & $\mathrm{~s}$ & $9 \mathrm{H}$ \\
\hline & 1,08 & $\mathrm{Si}\left(\mathrm{C}\left(\mathrm{CH}_{3}\right)_{3}\right)_{2}$ & $\mathrm{~s}$ & $18 \mathrm{H}$ \\
\hline & 1,14 & $\mathrm{C}\left(\mathrm{CH}_{3}\right)_{3}$ & $\mathrm{~s}$ & \\
\hline & 1,42 & $\mathrm{C}_{2}$ & $\mathrm{~s}$ & \\
\hline & 1,55 & $\mathrm{C}\left(\mathrm{C}_{3}\right)_{3}$ & $\mathrm{~s}$ & \\
\hline & 4,58 & $=\underline{\mathrm{CH}}$ & $\mathrm{s}$ & \\
\hline \multirow[t]{6}{*}{$\delta{ }^{13} \mathrm{C}[\mathrm{ppm}]$} & 3,8 & $=\mathrm{CSi}\left(\underline{\mathrm{CH}}_{3}\right)_{3}$ & $S$ & \\
\hline & 12,8 & $\underline{\mathrm{CH}}_{2}$ & $\mathrm{~s}$ & \\
\hline & 21,8 & $\mathrm{Si}\left(\underline{\mathrm{C}}\left(\mathrm{CH}_{3}\right)_{3}\right)_{2}$ & $\mathrm{~s}$ & \\
\hline & 28,7 & $\mathrm{C}\left(\underline{\mathrm{C}}_{3}\right)_{3}$ & $\mathrm{~s}$ & \\
\hline & 29,1 & $\mathrm{Si}\left(\mathrm{C}\left(\underline{\mathrm{CH}}_{3}\right)_{3}\right)_{2}$ & $\mathrm{~s}$ & \\
\hline & 31,3 & $\mathrm{C}\left(\underline{\mathrm{CH}}_{3}\right)_{3}$ & $\mathrm{~s}$ & \\
\hline
\end{tabular}




$\begin{array}{rrll}\delta{ }^{13} \mathrm{C}[\mathrm{ppm}] & 36,8 & \underline{\mathrm{C}}\left(\mathrm{CH}_{3}\right)_{3} & \mathrm{~s} \\ 38,8 & \underline{\mathrm{C}}\left(\mathrm{CH}_{3}\right)_{3} & \mathrm{~s} \\ 96,8 & =\underline{\mathrm{C}} \mathrm{H} & \mathrm{s} \\ 156,3 & \underline{\mathrm{CN}} & \mathrm{s} \\ 169,1 & \underline{\mathrm{CN}} & \mathrm{s}\end{array}$

$\delta^{29} \mathrm{Si}[\mathrm{ppm}] \quad-11,9 \quad=\mathrm{CSi}\left(\mathrm{CH}_{3}\right)_{3} \quad \mathrm{~s}$

$27,3 \underline{\mathrm{Si}}\left(\mathrm{C}\left(\mathrm{CH}_{3}\right)_{3}\right)_{2} \quad \mathrm{~s}$ 
Verbindung 26: 3,5-Di-tert-butyl-3-fluor-3,4-dihydro-2-(3,3-dimethylbut-1-en-2yl)-2H-1,2,3-diazasilol

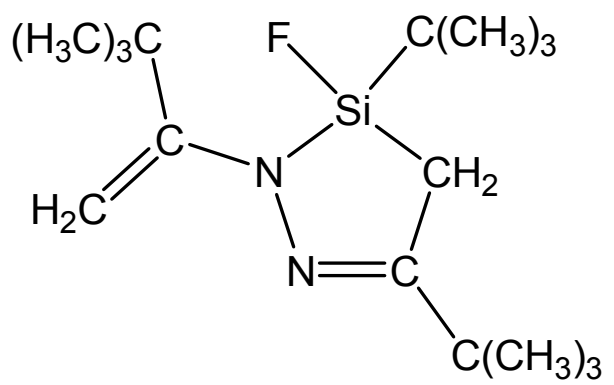

Summenformel: $\quad \mathrm{C}_{16} \mathrm{H}_{31} \mathrm{FN}_{2} \mathrm{Si}$

Molare Masse: $\quad 298,51 \mathrm{~g} / \mathrm{mol}$

MS (EI)

$\begin{array}{lcl}298 \mathrm{~g} / \mathrm{mol} & 27 \% & {[\mathrm{M}]^{+}} \\ 283 \mathrm{~g} / \mathrm{mol} & 9 \% & {[\mathrm{M}-\mathrm{Me}]^{+}} \\ 242 \mathrm{~g} / \mathrm{mol} & 60 \% & {\left[\mathrm{M}-\mathrm{C}_{4} \mathrm{H}_{8}\right]^{+}} \\ 57 \mathrm{~g} / \mathrm{mol} & 100 \% & {[\mathrm{Buu}]^{+}}\end{array}$

Siedepunkt: $\quad 58^{\circ} \mathrm{C} / 0,02 \mathrm{mbar}$

Ausbeute: $\quad 63 \%$

$\operatorname{NMR}\left(\mathrm{CDCl}_{3}\right)$ :

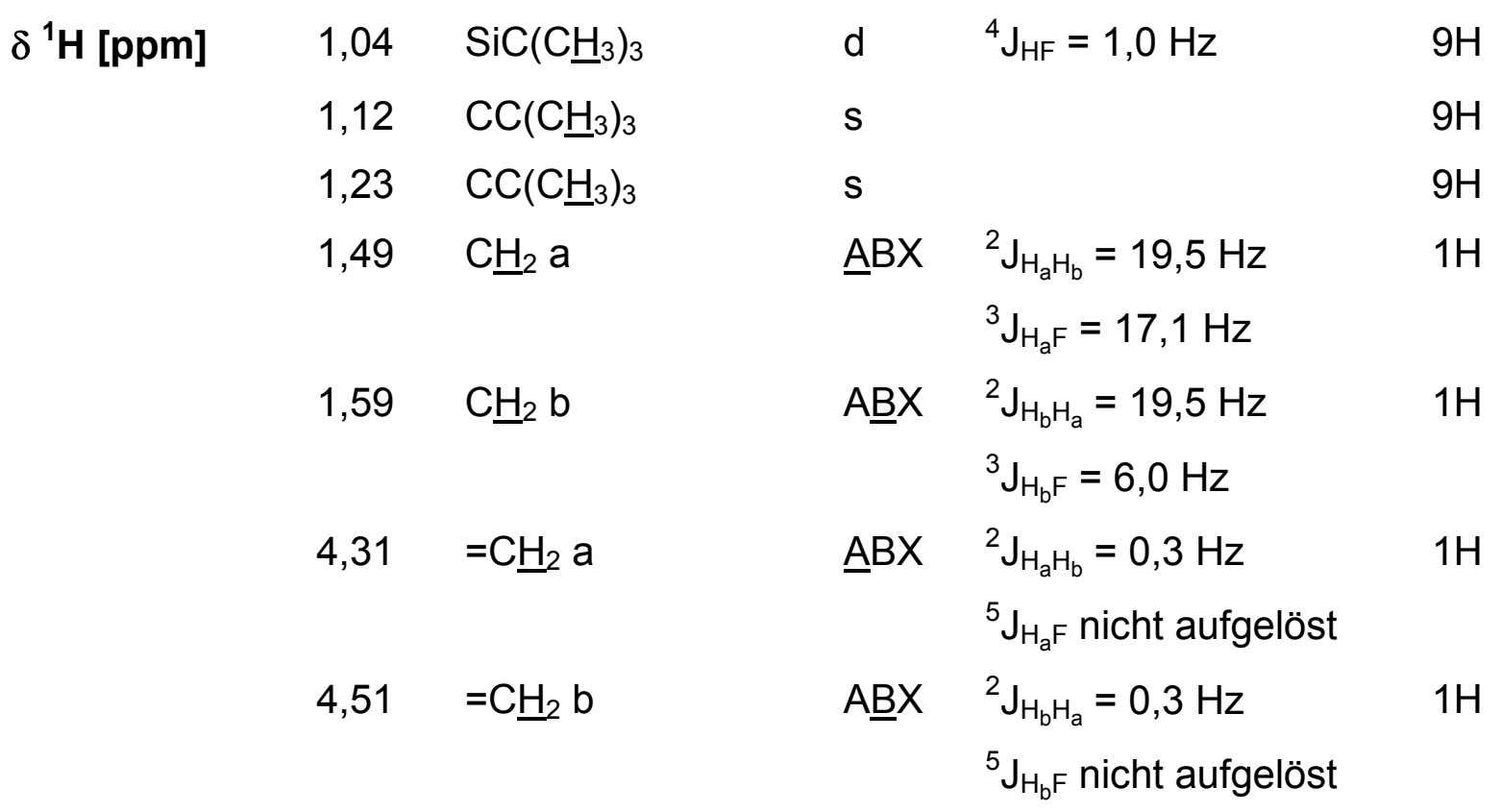




$\begin{array}{rrlll}\delta{ }^{13} \mathrm{C}[\mathrm{ppm}] & 10,7 & \underline{\mathrm{C}} \mathrm{H}_{2} & \mathrm{~d} & { }^{2} \mathrm{~J}_{\mathrm{CF}}=14,8 \mathrm{~Hz} \\ 18,6 & \mathrm{SiC}(\mathrm{CH})_{3} & \mathrm{~d} & { }^{2} \mathrm{~J}_{\mathrm{CF}}=20,1 \mathrm{~Hz} \\ 26,8 & \mathrm{SiC}(\underline{\mathrm{CH}})_{3} & \mathrm{~s} & \\ 28,5 & \mathrm{CC}\left(\underline{\mathrm{C}} \mathrm{H}_{3}\right)_{3} & \mathrm{~s} & \\ 30,2 & \mathrm{CC}\left(\underline{\mathrm{CH}}_{3}\right)_{3} & \mathrm{~s} & \\ 36,8 & \underline{\mathrm{C}}\left(\mathrm{CH}_{3}\right)_{3} & \mathrm{~s} & \\ 36,9 & \underline{\mathrm{C}}\left(\mathrm{CH}_{3}\right)_{3} & \mathrm{~d} & { }^{4} \mathrm{~J}_{\mathrm{CF}}=2,8 \mathrm{~Hz} \\ 93,1 & =\underline{\mathrm{C}} \mathrm{H}_{2} & \mathrm{~s} & \\ 156,8 & \underline{\mathrm{CN}} & \mathrm{d} & { }^{3} \mathrm{~J}_{\mathrm{CF}}=1,8 \mathrm{~Hz} \\ 159,4 & \underline{\mathrm{CN}} & \mathrm{d} & { }^{3} \mathrm{~J}_{\mathrm{CF}}=3,0 \mathrm{~Hz}\end{array}$

$\delta{ }^{19} \mathrm{~F}[\mathrm{ppm}] \quad 16,2 \quad \operatorname{SiFC}\left(\mathrm{CH}_{3}\right)_{3} \quad \mathrm{~s}$

$$
\begin{aligned}
\mathrm{ABX} \underline{X}^{*}{ }^{3} \mathrm{~J}_{\mathrm{FH}_{\mathrm{a}}} & =17,1 \mathrm{~Hz} \\
{ }^{3} \mathrm{~J}_{\mathrm{FH}_{\mathrm{b}}} & =6,0 \mathrm{~Hz}
\end{aligned}
$$

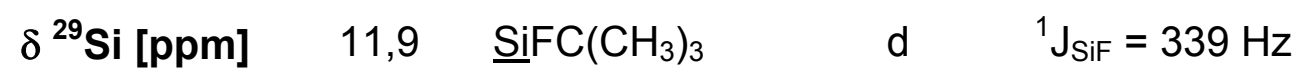

* : ${ }^{1} \mathrm{H}$-gekoppelt: Multiplett; $\mathrm{AB} \underline{\mathrm{X}}$ mit weiteren Kopplungen 
Verbindung 27: (5-tert-Butyl-3-fluor-3,4-dihydro-2-(3,3-dimethylbut-1-en-2-yl)2H-1,2,3-diazasilol-3-yl)-bis(trimethylsilyl)amin<smiles>C=C(N1N=C(C(C)(C)C)C[Si]1(F)N([SiH3])[SiH3])C(C)(C)C</smiles>

Summenformel: $\quad \mathrm{C}_{18} \mathrm{H}_{40} \mathrm{FN}_{3} \mathrm{Si}_{3}$

Molare Masse: $\quad 401,79 \mathrm{~g} / \mathrm{mol}$

MS (EI)

$\begin{array}{lrl}401 \mathrm{~g} / \mathrm{mol} & 9 \% & {[\mathrm{M}]^{+}} \\ 386 \mathrm{~g} / \mathrm{mol} & 19 \% & {[\mathrm{M}-\mathrm{Me}]^{+}} \\ 344 \mathrm{~g} / \mathrm{mol} & 100 \% & {[\mathrm{M}-\mathrm{tBu}]^{+}}\end{array}$

Siedepunkt: $\quad 104^{\circ} \mathrm{C} / 0,05 \mathrm{mbar}$

Ausbeute: $\quad 55 \%$

$\operatorname{NMR}\left(\mathrm{CDCl}_{3}\right)$ :

\begin{tabular}{|c|c|c|c|c|c|}
\hline$\delta{ }^{1} \mathrm{H}[\mathrm{ppm}]$ & 0,18 & $\left(\mathrm{Si}\left(\mathrm{C}_{3}\right)_{3}\right)_{2}$ & $d$ & ${ }^{5} J_{\mathrm{HF}}=1,1 \mathrm{~Hz}$ & $18 \mathrm{H}$ \\
\hline & 1,11 & $\mathrm{C}\left(\mathrm{C}_{3}\right)_{3}$ & $\mathrm{~s}$ & & $9 \mathrm{H}$ \\
\hline & 1,23 & $\mathrm{C}\left(\mathrm{C}_{3}\right)_{3}$ & $\mathrm{~s}$ & & $9 \mathrm{H}$ \\
\hline & 1,29 & $\mathrm{CH}_{2} \mathrm{a}$ & $\underline{A B X}$ & ${ }^{2} \mathrm{~J}_{\mathrm{H}_{\mathrm{a}} \mathrm{H}_{\mathrm{b}}}=19,7 \mathrm{~Hz}$ & $1 \mathrm{H}$ \\
\hline & & & & ${ }^{3} \mathrm{~J}_{\mathrm{H}_{\mathrm{a}} \mathrm{F}}=15,0 \mathrm{~Hz}$ & \\
\hline & 1,44 & $\mathrm{CH}_{2} \mathrm{~b}$ & $A \underline{B} X$ & ${ }^{2} \mathrm{~J}_{\mathrm{H}_{b} \mathrm{H}_{\mathrm{a}}}=19,7 \mathrm{~Hz}$ & $1 \mathrm{H}$ \\
\hline & & & & ${ }^{3} \mathrm{~J}_{\mathrm{H}_{b} \mathrm{~F}}=5,8 \mathrm{~Hz}$ & \\
\hline & 4,20 & $=\mathrm{CH}_{2} \mathrm{a}$ & $d$ & ${ }^{2} \mathrm{~J}_{\mathrm{H}_{\mathrm{a}} \mathrm{H}_{\mathrm{b}}}=0,7 \mathrm{~Hz}$ & $1 \mathrm{H}$ \\
\hline & 4,43 & $=\mathrm{C}_{2} \mathrm{~b}$ & $d v d$ & ${ }^{2} \mathrm{~J}_{\mathrm{H}_{\mathrm{b}} \mathrm{H}_{\mathrm{a}}}=0,7 \mathrm{~Hz}$ & $1 \mathrm{H}$ \\
\hline & & & & ${ }^{5} \mathrm{~J}_{\mathrm{H}_{b} \mathrm{~F}}=0,6 \mathrm{~Hz}$ & \\
\hline
\end{tabular}




$\begin{array}{rrlll}\delta{ }^{13} \mathrm{C} \text { [ppm] } & 3,9 & \left(\mathrm{Si}\left(\underline{\mathrm{CH}}_{3}\right)_{3}\right)_{2} & \mathrm{~d} & { }^{4} \mathrm{~J}_{\mathrm{CF}}=2,3 \mathrm{~Hz} \\ 12,9 & \underline{\mathrm{C}} \mathrm{H}_{2} & \mathrm{~d} & { }^{3} \mathrm{~J}_{\mathrm{CF}}=20,3 \mathrm{~Hz} \\ 28,5 & \underline{\mathrm{C}}\left(\underline{\mathrm{C}} \mathrm{H}_{3}\right)_{3} & \mathrm{~s} & \\ 29,9 & \mathrm{C}\left(\underline{\mathrm{C}} \mathrm{H}_{3}\right)_{3} & \mathrm{~s} & \\ 36,7 & \underline{\mathrm{C}}\left(\mathrm{CH}_{3}\right)_{3} & \mathrm{~d} & { }^{4} \mathrm{~J}_{\mathrm{CF}}=1,0 \mathrm{~Hz} \\ 37,1 & \underline{\mathrm{C}}\left(\mathrm{CH}_{3}\right)_{3} & \mathrm{~d} & { }^{4} \mathrm{~J}_{\mathrm{CF}}=0,3 \mathrm{~Hz} \\ 89,1 & =\underline{\mathrm{C}} \mathrm{H}_{2} & \mathrm{~d} & { }^{4} \mathrm{~J}_{\mathrm{CF}}=0,6 \mathrm{~Hz} \\ 155,7 & \underline{\mathrm{CN}} & \mathrm{d} & { }^{3} \mathrm{~J}_{\mathrm{CF}}=2,8 \mathrm{~Hz} \\ 157,9 & \underline{\mathrm{CN}} & \mathrm{d} & { }^{3} \mathrm{~J}_{\mathrm{CF}}=2,0 \mathrm{~Hz}\end{array}$

$\delta^{19} \mathrm{~F}[\mathrm{ppm}] \quad 36,6 \quad \mathrm{SiE}$

S

$$
\begin{aligned}
\mathrm{ABX} \underline{X}^{*}{ }^{3} \mathrm{~J}_{\mathrm{FH}_{\mathrm{a}}} & =15,0 \mathrm{~Hz} \\
{ }^{3} \mathrm{~J}_{\mathrm{FH}_{\mathrm{b}}} & =5,8 \mathrm{~Hz}
\end{aligned}
$$

$\delta^{29} \mathrm{Si}[\mathrm{ppm}] \quad-13,9 \quad \mathrm{NSiF}$

d $\quad{ }^{1} \mathrm{~J}_{\mathrm{SiF}}=282 \mathrm{~Hz}$

$5,6 \quad\left(\underline{\mathrm{Si}}\left(\mathrm{CH}_{3}\right)_{3}\right)_{2}$

d $\quad{ }^{3} \mathrm{~J}_{\mathrm{SiF}}=4,3 \mathrm{~Hz}$

* : ${ }^{1} \mathrm{H}$-gekoppelt: Multiplett; ABX mit weiteren Kopplungen 
Verbindung 28: $\quad N$-(5-tert-Butyl-3-fluor-3,4-dihydro-2-(3,3-dimethylbut-1-en-2yl)-2H-1,2,3-diazasilol-3-yl)-2-methyl-N-(trimethylsilyl)propan2-amin<smiles>C=C(N1N=C(C(C)(C)C)C[Si]1(F)N([Si])C(C)(C)C)C(C)(C)C</smiles>

Summenformel: $\quad \mathrm{C}_{19} \mathrm{H}_{40} \mathrm{FN}_{3} \mathrm{Si}_{2}$

Molare Masse: $\quad 385,71 \mathrm{~g} / \mathrm{mol}$

MS (EI)

$385 \mathrm{~g} / \mathrm{mol}$
$370 \mathrm{~g} / \mathrm{mol}$
$328 \mathrm{~g} / \mathrm{mol}$
$314 \mathrm{~g} / \mathrm{mol}$

$\begin{aligned} 12 \% & {[\mathrm{M}]^{+} } \\ 18 \% & {[\mathrm{M}-\mathrm{Me}]^{+} } \\ 60 \% & {[\mathrm{M}-t \mathrm{Bu}]^{+} } \\ 100 \% & {[\mathrm{M}-\mathrm{N} t \mathrm{Bu}]^{+} }\end{aligned}$

Siedepunkt: $\quad 89^{\circ} \mathrm{C} / 0,06 \mathrm{mbar}$

Ausbeute: $\quad 68 \%$

$\operatorname{NMR}\left(\mathrm{C}_{6} \mathrm{D}_{6}\right)$ :

$\begin{array}{clllll}\delta{ }^{1} \mathrm{H} \text { [ppm] } & 0,24 & \mathrm{NSi}\left(\mathrm{CH}_{3}\right)_{3} & \mathrm{~d} & { }^{5} \mathrm{~J}_{\mathrm{HF}}=2,0 \mathrm{~Hz} & 9 \mathrm{H} \\ & 1,11 & \mathrm{CC}\left(\mathrm{C}_{3}\right)_{3} & \mathrm{~s} & & 9 \mathrm{H} \\ 1,23 & \mathrm{CC}\left(\mathrm{CH}_{3}\right)_{3} & \mathrm{~s} & & 9 \mathrm{H} \\ 1,31 & \mathrm{NC}\left(\mathrm{C}_{3}\right)_{3} & \mathrm{~d} & { }^{5} \mathrm{~J}_{\mathrm{HF}}=0,5 \mathrm{~Hz} & 9 \mathrm{H} \\ 1,34 & \mathrm{C} \underline{\mathrm{H}}_{2} \mathrm{a} & \underline{\mathrm{ABX}} & { }^{2} \mathrm{~J}_{\mathrm{H}_{\mathrm{a}} \mathrm{H}_{\mathrm{b}}}=19,6 \mathrm{~Hz} & 1 \mathrm{H} \\ & & & { }^{3} \mathrm{~J}_{\mathrm{H}_{\mathrm{a}} \mathrm{F}}=12,8 \mathrm{~Hz} & \\ 1,43 & \mathrm{C} \underline{\mathrm{H}}_{2} \mathrm{~b} & \mathrm{ABXX} & { }^{2} \mathrm{~J}_{\mathrm{H}_{\mathrm{b}} \mathrm{H}_{\mathrm{a}}}=19,6 \mathrm{~Hz} & 1 \mathrm{H} \\ & & & { }^{3} \mathrm{~J}_{\mathrm{H}_{\mathrm{b}} \mathrm{F}}=7,0 \mathrm{~Hz} & \\ 4,19 & =\underline{\mathrm{C}}_{2} \mathrm{a} & \mathrm{d} & { }^{2} \mathrm{~J}_{\mathrm{H}_{\mathrm{a}} \mathrm{H}_{\mathrm{b}}}=0,5 \mathrm{~Hz} & 1 \mathrm{H} \\ 4,50 & =\mathrm{C} \underline{\mathrm{H}}_{2} \mathrm{~b} & \mathrm{~d} & { }^{2} \mathrm{~J}_{\mathrm{H}_{\mathrm{b}} \mathrm{H}_{\mathrm{a}}}=0,5 \mathrm{~Hz} & 1 \mathrm{H}\end{array}$




$\begin{array}{rrlll}\delta{ }^{13} \mathrm{C} \text { [ppm] } & 5,4 & \mathrm{NSi}\left(\underline{\mathrm{C}} \mathrm{H}_{3}\right)_{3} & \mathrm{~d} & { }^{4} \mathrm{~J}_{\mathrm{CF}}=4,2 \mathrm{~Hz} \\ 14,0 & \underline{\mathrm{C}} \mathrm{H}_{2} & \mathrm{~d} & { }^{2} \mathrm{~J}_{\mathrm{CF}}=19,9 \mathrm{~Hz} \\ 28,4 & \mathrm{CC}\left(\underline{\mathrm{C}} \mathrm{H}_{3}\right)_{3} & \mathrm{~s} & \\ 29,8 & \mathrm{CC}\left(\underline{\mathrm{C}} \mathrm{H}_{3}\right)_{3} & \mathrm{~s} & \\ 33,4 & \mathrm{NC}\left(\underline{\mathrm{CH}}_{3}\right)_{3} & \mathrm{~d} & { }^{4} \mathrm{~J}_{\mathrm{CF}}=2,3 \mathrm{~Hz} \\ 36,7 & \underline{\mathrm{C}}\left(\mathrm{CH}_{3}\right)_{3} & \mathrm{~d} & { }^{4} \mathrm{~J}_{\mathrm{CF}}=0,9 \mathrm{~Hz} \\ 37,0 & \underline{\mathrm{C}}\left(\mathrm{CH}_{3}\right)_{3} & \mathrm{~s} & \\ 54,6 & \underline{\mathrm{N}}\left(\mathrm{CH}_{3}\right)_{3} & \mathrm{~s} & \\ 88,5 & =\underline{\mathrm{C}} \mathrm{H}_{2} & \mathrm{~s} & \\ 155,6 & \underline{\mathrm{CN}} & \mathrm{d} & { }^{3} \mathrm{~J}_{\mathrm{CF}}=3,3 \mathrm{~Hz} \\ 157,9 & \underline{\mathrm{CN}} & \mathrm{d} & { }^{3} \mathrm{~J}_{\mathrm{CF}}=1,9 \mathrm{~Hz}\end{array}$

$\delta^{19} \mathrm{~F}[\mathrm{ppm}] \quad 33,8 \quad$ SiFN $\quad S$

$$
\begin{aligned}
\mathrm{ABX} \underline{X}^{*}{ }^{3} \mathrm{~J}_{\mathrm{FH}_{\mathrm{a}}} & =12,8 \mathrm{~Hz} \\
{ }^{3} \mathrm{~J}_{\mathrm{FH}_{\mathrm{b}}} & =7,0 \mathrm{~Hz}
\end{aligned}
$$

$\delta^{29} \mathrm{Si}[\mathrm{ppm}] \quad-12,6 \quad \underline{\mathrm{SiFN}} \quad \mathrm{d} \quad{ }^{1} \mathrm{~J}_{\mathrm{SiF}}=276 \mathrm{~Hz}$

$5,4 \quad \mathrm{NSi}\left(\mathrm{CH}_{3}\right)_{3} \quad$ d $\quad{ }^{3} \mathrm{~J}_{\mathrm{SiF}}=4,1 \mathrm{~Hz}$

* : ${ }^{1} \mathrm{H}$-gekoppelt: Multiplett; $\mathrm{AB} \underline{X}$ mit weiteren Kopplungen 
Verbindung 29: 3,5-Di-tert-butyl-3-fluor-3,4-dihydro-2-(3,3-dimethyl-1(trimethylsilyl)but-1-en-2-yl)-4-(trimethylsilyl)-2H-1,2,3diazasilol<smiles>CC(C)(C)C1=NN(/C(=C\[SiH3])C(C)(C)C)[Si](F)(C(C)(C)C)C1[SiH3]</smiles>

Summenformel: $\quad \mathrm{C}_{22} \mathrm{H}_{47} \mathrm{FN}_{2} \mathrm{Si}_{3}$

Molare Masse: $\quad 442,88 \mathrm{~g} / \mathrm{mol}$

MS (EI)

$442 \mathrm{~g} / \mathrm{mol}$

$30 \% \quad[\mathrm{M}]^{+}$

$427 \mathrm{~g} / \mathrm{mol}$

$30 \% \quad[\mathrm{M}-\mathrm{Me}]^{+}$

$385 \mathrm{~g} / \mathrm{mol}$

$83 \% \quad[\mathrm{M}-\mathrm{tBu}]^{+}$

$329 \mathrm{~g} / \mathrm{mol}$

$100 \% \quad\left[\mathrm{M}-\mathrm{BBu}-\mathrm{C}_{4} \mathrm{H}_{8}\right]^{+}$

Siedepunkt: $\quad 120^{\circ} \mathrm{C} / 0,5 \mathrm{mbar}$

Ausbeute: $\quad 69 \%$

Isomerengemisch im

Verhältnis $A: B=2: 1$

$\operatorname{NMR}\left(\mathrm{CDCl}_{3}\right)$ :

Isomer A:

$\delta{ }^{1} \mathrm{H}$ [ppm]

$0,13 \quad \mathrm{CSi}\left(\mathrm{CH}_{3}\right)_{3}$

$\mathrm{s}$

$9 \mathrm{H}$

$0,21=\mathrm{CSi}\left(\mathrm{C}_{\mathrm{H}_{3}}\right)_{3}$

d

${ }^{7} \mathrm{~J}_{\mathrm{HF}}=1,3 \mathrm{~Hz}$

$9 \mathrm{H}$

$0,96 \quad \operatorname{SiC}\left(\mathrm{CH}_{3}\right)_{3}$

d

$$
{ }^{4} \mathrm{~J}_{\mathrm{HF}}=0,9 \mathrm{~Hz}
$$

$9 \mathrm{H}$

$1,15 \quad \mathrm{CC}\left(\mathrm{CH}_{3}\right)_{3}$

s

$9 \mathrm{H}$

$1,30 \quad \mathrm{CC}\left(\mathrm{CH}_{3}\right)_{3}$

$\mathrm{S}$

$9 \mathrm{H}$

$1,44 \quad \mathrm{C} \underline{\mathrm{H}}$

d $\quad{ }^{3} \mathrm{~J}_{\mathrm{HF}}=11,2 \mathrm{~Hz}$

$1 \mathrm{H}$

$4,92=\mathrm{C} \underline{\mathrm{H}}$

d $\quad{ }^{5} \mathrm{~J}_{\mathrm{HF}}=1,7 \mathrm{~Hz}$

$1 \mathrm{H}$
$\delta{ }^{13} \mathrm{C}[\mathrm{ppm}]$
1,8 $\mathrm{CSi}\left(\underline{\mathrm{CH}}_{3}\right)_{3}$
d
${ }^{4} \mathrm{~J}_{\mathrm{CF}}=2,7 \mathrm{~Hz}$
$3,0=\mathrm{CSi}\left(\underline{\mathrm{CH}}_{3}\right)_{3}$
$\mathrm{S}$ 


\begin{tabular}{|c|c|c|c|c|}
\hline \multirow[t]{10}{*}{$\delta{ }^{13} \mathrm{C}[\mathrm{ppm}]$} & 18,5 & $\underline{\mathrm{C}} \mathrm{H}$ & $d$ & ${ }^{2} \mathrm{~J}_{\mathrm{CF}}=13,5 \mathrm{~Hz}$ \\
\hline & 20,5 & $\mathrm{SiC}(\mathrm{CH})_{3}$ & $\mathrm{~d}$ & ${ }^{2} \mathrm{~J}_{\mathrm{CF}}=22,6 \mathrm{~Hz}$ \\
\hline & 26,5 & $\mathrm{SiC}(\underline{\mathrm{CH}})_{3}$ & $\mathrm{~s}$ & \\
\hline & 30,0 & $\mathrm{CC}\left(\underline{\mathrm{CH}}_{3}\right)_{3}$ & $\mathrm{~s}$ & \\
\hline & 30,9 & $\mathrm{CC}\left(\underline{\mathrm{CH}}_{3}\right)_{3}$ & $\mathrm{~s}$ & \\
\hline & 37,4 & $\mathrm{C} \underline{\mathrm{C}}\left(\mathrm{CH}_{3}\right)_{3}$ & $\mathrm{~s}$ & \\
\hline & 38,3 & $\mathrm{C} \underline{\mathrm{C}}\left(\mathrm{CH}_{3}\right)_{3}$ & $\mathrm{~d}$ & ${ }^{4} \mathrm{~J}_{\mathrm{CF}}=3,0 \mathrm{~Hz}$ \\
\hline & 103,2 & $=\underline{\mathrm{C}} \mathrm{H}$ & $\mathrm{s}$ & \\
\hline & 156,2 & $\underline{\mathrm{CN}}$ & $d$ & ${ }^{3} \mathrm{~J}_{\mathrm{CF}}=4,3 \mathrm{~Hz}$ \\
\hline & 166,0 & $\underline{\mathrm{C} N}$ & $d$ & ${ }^{3} \mathrm{~J}_{\mathrm{CF}}=2,6 \mathrm{~Hz}$ \\
\hline$\delta^{19} \mathrm{~F}$ [ppm] & 19,4 & $\operatorname{SiFC}\left(\mathrm{CH}_{3}\right)_{3}$ & $d$ & ${ }^{3} \mathrm{~J}_{\mathrm{FH}}=11,2 \mathrm{~Hz}$ \\
\hline \multirow[t]{3}{*}{$\delta^{29} \mathrm{Si}[\mathrm{ppm}]$} & $-11,9$ & $=\mathrm{CS} \underline{\underline{i}}\left(\mathrm{CH}_{3}\right)_{3}$ & $S$ & \\
\hline & 0,1 & $\mathrm{CSi}\left(\mathrm{CH}_{3}\right)_{3}$ & $d$ & ${ }^{3} \mathrm{~J}_{\mathrm{SiF}}=5,9 \mathrm{~Hz}$ \\
\hline & 18,0 & $\underline{\operatorname{SiF}} \mathrm{C}\left(\mathrm{CH}_{3}\right)_{3}$ & $d$ & ${ }^{1} \mathrm{~J}_{\mathrm{SiF}}=329 \mathrm{~Hz}$ \\
\hline
\end{tabular}


NMR (CDCl $\left.{ }_{3}\right)$ :

Isomer B:

$\delta{ }^{1} \mathrm{H}$ [ppm]

$\begin{array}{ll}0,05 & \mathrm{CSi}\left(\mathrm{CH}_{3}\right)_{3} \\ 0,26 & =\mathrm{CSi}\left(\mathrm{C}_{3}\right)_{3} \\ 0,98 & \mathrm{SiC}\left(\mathrm{C}_{3}\right)_{3} \\ 1,16 & \mathrm{CC}\left(\mathrm{CH}_{3}\right)_{3} \\ 1,20 & \mathrm{CC}\left(\underline{\mathrm{CH}}_{3}\right)_{3} \\ 1,45 & \mathrm{C} \underline{\mathrm{H}} \\ 5,05 & =\mathrm{C} \underline{\mathrm{H}}\end{array}$

$\begin{array}{lll}\text { d } & { }^{5} \mathrm{~J}_{\mathrm{HF}}=1,5 \mathrm{~Hz} & 9 \mathrm{H} \\ \mathrm{d} & { }^{7} \mathrm{~J}_{\mathrm{HF}}=0,8 \mathrm{~Hz} & 9 \mathrm{H} \\ \mathrm{d} & { }^{4} \mathrm{~J}_{\mathrm{HF}}=1,0 \mathrm{~Hz} & 9 \mathrm{H} \\ \mathrm{s} & & 9 \mathrm{H} \\ \mathrm{s} & & 9 \mathrm{H} \\ \mathrm{d} & { }^{3} \mathrm{~J}_{\mathrm{HF}}=15,9 \mathrm{~Hz} & 1 \mathrm{H} \\ \mathrm{s} & & 1 \mathrm{H}\end{array}$

$\delta{ }^{13} \mathrm{C}[\mathrm{ppm}]$

$\begin{array}{rl}1,0 & \mathrm{CSi}\left(\underline{\mathrm{C}} \mathrm{H}_{3}\right)_{3} \\ 2,7 & =\mathrm{CSi}\left(\underline{\mathrm{C}} \mathrm{H}_{3}\right)_{3} \\ 18,6 & \underline{\mathrm{CH}} \\ 20,1 & \mathrm{Si} \underline{\mathrm{C}}(\mathrm{CH})_{3} \\ 27,54 & \mathrm{SiC}(\underline{\mathrm{CH}})_{3} \\ 29,7 & \mathrm{CC}\left(\underline{\mathrm{C}} \mathrm{H}_{3}\right)_{3} \\ 31,3 & \mathrm{CC}\left(\underline{\mathrm{C}} \mathrm{H}_{3}\right)_{3} \\ 38,0 & \mathrm{C} \underline{\mathrm{C}}\left(\mathrm{CH}_{3}\right)_{3} \\ 39,8 & \mathrm{C} \underline{\mathrm{C}}\left(\mathrm{CH}_{3}\right)_{3} \\ 112,2 & =\underline{\mathrm{C}} \mathrm{H} \\ 157,2 & \underline{\mathrm{CN}} \\ 165,2 & \underline{\mathrm{CN}}\end{array}$

$\delta{ }^{19} \mathrm{~F}[\mathrm{ppm}] \quad 31,9 \quad \operatorname{SiFC}\left(\mathrm{CH}_{3}\right)_{3}$

d $\quad{ }^{3} \mathrm{~J}_{\mathrm{FH}}=15,9 \mathrm{~Hz}$

$\delta^{29} \mathrm{Si}[\mathrm{ppm}]$

$\begin{array}{lrlll}\delta{ }^{19} \mathrm{~F} \text { [ppm] } & 31,9 & \operatorname{SiEC}\left(\mathrm{CH}_{3}\right)_{3} & \mathrm{~d} & { }^{3} \mathrm{~J}_{\mathrm{FH}}=15,9 \mathrm{~Hz} \\ \delta^{29} \mathrm{Si}[\mathrm{ppm}] & -9,5 & =\mathrm{CSi}\left(\mathrm{CH}_{3}\right)_{3} & \mathrm{~d} & { }^{5} \mathrm{~J}_{\mathrm{SiF}}=2,0 \mathrm{~Hz} \\ & -0,7 & \mathrm{CS}\left(\mathrm{CH}_{3}\right)_{3} & \mathrm{~d} & { }^{3} \mathrm{~J}_{\mathrm{SiF}}=5,5 \mathrm{~Hz} \\ & 14,1 & \underline{\operatorname{SiFC}}\left(\mathrm{CH}_{3}\right)_{3} & \mathrm{~d} & { }^{1} \mathrm{~J}_{\mathrm{SiF}}=328 \mathrm{~Hz}\end{array}$

d

${ }^{5} \mathrm{~J}_{\mathrm{SiF}}=2,0 \mathrm{~Hz}$

d

${ }^{3} \mathrm{~J}_{\mathrm{SiF}}=5,5 \mathrm{~Hz}$

d

d $\quad{ }^{4} \mathrm{~J}_{\mathrm{CF}}=2,1 \mathrm{~Hz}$

d $\quad{ }^{3} \mathrm{~J}_{\mathrm{CF}}=2,5 \mathrm{~Hz}$

d $\quad{ }^{3} \mathrm{~J}_{\mathrm{CF}}=1,9 \mathrm{~Hz}$

$\begin{array}{ll}\text { d } & { }^{6} \mathrm{~J}_{\mathrm{CF}}=2,1 \mathrm{~Hz} \\ \text { d } & { }^{2} \mathrm{~J}_{\mathrm{CF}}=16,6 \mathrm{~Hz} \\ \text { d } & { }^{2} \mathrm{~J}_{\mathrm{CF}}=24,3 \mathrm{~Hz}\end{array}$

s

$\mathrm{s}$ 
Verbindung 30: 5-tert-Butyl-3-fluor-3,4-dihydro-2-(3,3-dimethyl-1-(trimethylsilyl)but-1-en-2-yl)-4-(trimethylsilyl)-3-phenyl-2H-1,2,3diazasilol

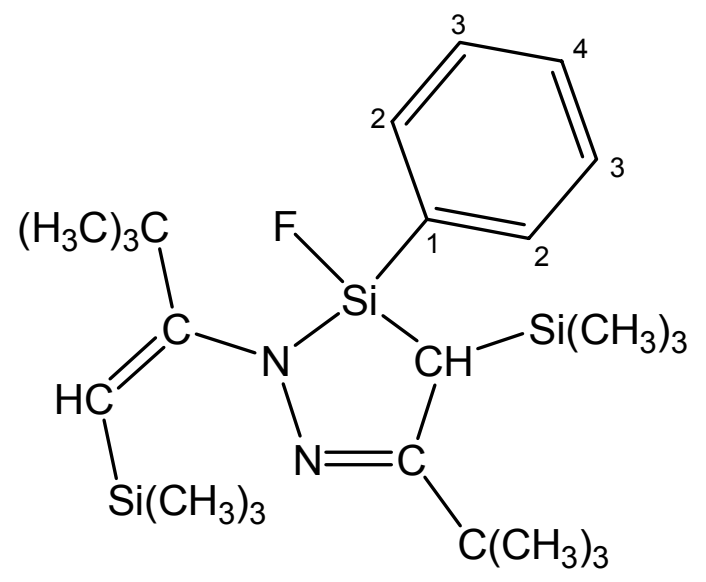

Summenformel: $\quad \mathrm{C}_{24} \mathrm{H}_{43} \mathrm{FN}_{2} \mathrm{Si}_{3}$

Molare Masse: $\quad 462,87 \mathrm{~g} / \mathrm{mol}$

MS (EI)

$462 \mathrm{~g} / \mathrm{mol}$

$29 \% \quad[\mathrm{M}]^{+}$

$447 \mathrm{~g} / \mathrm{mol}$

$34 \% \quad[\mathrm{M}-\mathrm{Me}]^{+}$

$405 \mathrm{~g} / \mathrm{mol}$

$50 \% \quad[\mathrm{M}-\mathrm{tBu}]^{+}$

$349 \mathrm{~g} / \mathrm{mol}$

$100 \% \quad\left[\mathrm{M}-\mathrm{tBu}-\mathrm{C}_{4} \mathrm{H}_{8}\right]^{+}$

Siedepunkt: $\quad 116{ }^{\circ} \mathrm{C} / 0,009 \mathrm{mbar}$

Ausbeute:

$61 \%$

NMR ( $\left.\mathrm{CDCl}_{3}\right)$ :

$\delta{ }^{1} \mathrm{H}[\mathrm{ppm}]$

$\begin{array}{rl}0,00 & \mathrm{CSi}\left(\underline{\mathrm{C}}_{3}\right)_{3} \\ 0,24 & =\mathrm{CSi}\left(\mathrm{C}_{3}\right)_{3} \\ 0,93 & \mathrm{C}\left(\mathrm{C}_{3}\right)_{3} \\ 1,21 & \mathrm{C}\left(\underline{\mathrm{C}}_{3}\right)_{3} \\ 1,79 & \mathrm{C} \underline{\mathrm{H}} \\ 5,25 & =\mathrm{C} \underline{\mathrm{H}} \\ 7,3-7,6 & \mathrm{C}_{6} \underline{\mathrm{H}}_{5}\end{array}$

d

$$
{ }^{5} \mathrm{~J}_{\mathrm{HF}}=0,8 \mathrm{~Hz}
$$

$9 \mathrm{H}$

d

$$
{ }^{7} \mathrm{~J}_{\mathrm{HF}}=1,2 \mathrm{~Hz}
$$

$9 \mathrm{H}$

$\mathrm{S}$

$9 \mathrm{H}$

$\mathrm{S}$

$9 \mathrm{H}$

d $\quad{ }^{3} \mathrm{~J}_{\mathrm{HF}}=19,2 \mathrm{~Hz}$

$1 \mathrm{H}$

$\mathrm{S}$

$1 \mathrm{H}$

$\mathrm{m}$

$5 \mathrm{H}$

$\delta{ }^{13} \mathrm{C}[\mathrm{ppm}]$

$$
\begin{array}{ll}
0,3 & \mathrm{CSi}\left(\underline{\mathrm{CH}}_{3}\right)_{3} \\
2,2 & =\mathrm{CSi}\left(\underline{\mathrm{CH}}_{3}\right)_{3}
\end{array}
$$

d $\quad{ }^{4} \mathrm{~J}_{\mathrm{CF}}=2,1 \mathrm{~Hz}$

d $\quad{ }^{6} \mathrm{~J}_{\mathrm{CF}}=1,6 \mathrm{~Hz}$ 


\begin{tabular}{|c|c|c|c|c|}
\hline \multirow[t]{12}{*}{$\delta{ }^{13} \mathrm{C}[\mathrm{ppm}]$} & 20,7 & $\underline{\mathrm{C}} \mathrm{H}$ & $d$ & \multirow[t]{4}{*}{${ }^{2} \mathrm{~J}_{\mathrm{CF}}=17,7 \mathrm{~Hz}$} \\
\hline & 29,3 & $\mathrm{C}\left(\underline{\mathrm{CH}}_{3}\right)_{3}$ & $\mathrm{~s}$ & \\
\hline & 30,3 & $\mathrm{C}\left(\underline{\mathrm{CH}}_{3}\right)_{3}$ & $\mathrm{~s}$ & \\
\hline & 37,8 & $\underline{\mathrm{C}}\left(\mathrm{CH}_{3}\right)_{3}$ & $\mathrm{~s}$ & \\
\hline & 39,1 & $\underline{\mathrm{C}}\left(\mathrm{CH}_{3}\right)_{3}$ & $d$ & \multirow[t]{4}{*}{${ }^{4} \mathrm{~J}_{\mathrm{CF}}=2,1 \mathrm{~Hz}$} \\
\hline & 118,4 & $=\underline{\mathrm{CH}}$ & $\mathrm{s}$ & \\
\hline & 127,9 & $\mathrm{Ph}(\underline{\mathrm{C}} 3 / 2)$ & $\mathrm{s}$ & \\
\hline & 130,9 & $\mathrm{Ph}(\underline{\mathrm{C}} 4)$ & $\mathrm{s}$ & \\
\hline & 134,7 & $\mathrm{Ph}(\underline{\mathrm{C}} 2 / 3)$ & $d$ & ${ }^{3} \mathrm{~J}_{\mathrm{CF}}=1,6 \mathrm{~Hz}$ \\
\hline & 136,3 & $\mathrm{Ph}(\underline{\mathrm{C}} 1)$ & $d$ & ${ }^{2} \mathrm{~J}_{\mathrm{CF}}=7,8 \mathrm{~Hz}$ \\
\hline & 157,2 & $\underline{\mathrm{CN}}$ & $d$ & ${ }^{3} \mathrm{~J}_{\mathrm{CF}}=1,4 \mathrm{~Hz}$ \\
\hline & 164,1 & $\underline{\mathrm{C} N}$ & $d$ & ${ }^{3} \mathrm{~J}_{\mathrm{CF}}=1,7 \mathrm{~Hz}$ \\
\hline \multirow[t]{2}{*}{$\delta^{19} \mathrm{~F}[\mathrm{ppm}]$} & 35,1 & PhSiF & $d$ & ${ }^{3} \mathrm{~J}_{\mathrm{FH}}=19,2 \mathrm{~Hz}$ \\
\hline & & & & ${ }^{1} \mathrm{~J}_{\mathrm{FSi}}=305 \mathrm{~Hz}$ \\
\hline \multirow[t]{3}{*}{$\delta{ }^{29} \mathrm{Si}$ [ppm] } & $-9,2$ & $=\mathrm{CS} \underline{\underline{S i}}\left(\mathrm{CH}_{3}\right)_{3}$ & $d$ & ${ }^{5} \mathrm{~J}_{\mathrm{SiF}}=0,2 \mathrm{~Hz}$ \\
\hline & $-0,3$ & $\underline{\text { SiFPh }}$ & $d$ & ${ }^{1} \mathrm{~J}_{\mathrm{SiF}}=305 \mathrm{~Hz}$ \\
\hline & $-0,3$ & $\mathrm{CSi}\left(\mathrm{CH}_{3}\right)_{3}$ & $d$ & ${ }^{3} \mathrm{~J}_{\mathrm{SiF}}=4,8 \mathrm{~Hz}$ \\
\hline
\end{tabular}


Verbindung 31: (5-tert-Butyl-3-fluor-3,4-dihydro-2-(3,3-dimethyl-1-

(trimethylsilyl)but-1-en-2-yl)-4-(trimethylsilyl)-2H-1,2,3diazasilol-3-yl)-bis(trimethylsilyl)amine<smiles>CC(C)(C)C1=NN(/C(=C\[SiH3])C(C)(C)C)[Si](F)(N([SiH3])[SiH3])C1[SiH3]</smiles>

Summenformel: $\quad \mathrm{C}_{24} \mathrm{H}_{56} \mathrm{FN}_{3} \mathrm{Si}_{5}$

Molare Masse: $\quad 546,15 \mathrm{~g} / \mathrm{mol}$

MS (EI)

$545 \mathrm{~g} / \mathrm{mol}$

$20 \% \quad[\mathrm{M}]^{+}$

$530 \mathrm{~g} / \mathrm{mol}$

$28 \% \quad[\mathrm{M}-\mathrm{Me}]^{+}$

$488 \mathrm{~g} / \mathrm{mol}$

$100 \% \quad[\mathrm{M}-\mathrm{tBu}]^{+}$

Siedepunkt: $\quad 152{ }^{\circ} \mathrm{C} / 0,04 \mathrm{mbar}$

Ausbeute: $\quad 58 \%$

Isomerengemisch

Verhältnis $A: B=5: 1$

$\operatorname{NMR}\left(\mathrm{CDCl}_{3}\right)$ :

Isomer A:

$\delta{ }^{1} \mathrm{H}[\mathrm{ppm}]$

$0,17=\mathrm{CSi}\left(\underline{\mathrm{C}}_{3}\right)_{3}$

$\mathrm{s}$

$9 \mathrm{H}$

$0,20 \mathrm{~N}\left(\mathrm{Si}\left(\mathrm{CH}_{3}\right)_{3}\right)_{2}$

d

${ }^{5} \mathrm{~J}_{\mathrm{HF}}=1,3 \mathrm{~Hz}$

$18 \mathrm{H}$

$0,22 \quad \mathrm{CSi}\left(\mathrm{CH}_{3}\right)_{3}$

d

${ }^{5} \mathrm{~J}_{\mathrm{HF}}=1,3 \mathrm{~Hz}$

$9 \mathrm{H}$

$1,17 \quad \mathrm{C}\left(\mathrm{CH}_{3}\right)_{3}$

$\mathrm{S}$

$9 \mathrm{H}$

$1,24 \quad \mathrm{C}\left(\mathrm{CH}_{3}\right)_{3}$

S

$9 \mathrm{H}$

$1,24 \quad \mathrm{CH}$

d ${ }^{3} \mathrm{~J}_{\mathrm{HF}}=12,4 \mathrm{~Hz}$

$1 \mathrm{H}$

$5,03=\mathrm{C} \underline{\mathrm{H}}$

$\mathrm{s}$

${ }^{5} \mathrm{~J}_{\mathrm{HF}}=1,1 \mathrm{~Hz}$

$1 \mathrm{H}$

$\delta{ }^{13} \mathrm{C}[\mathrm{ppm}]$

2,2 $\mathrm{CSi}\left(\underline{\mathrm{CH}}_{3}\right)_{3}$

d $\quad{ }^{4} J_{C F}=2,4 \mathrm{~Hz}$

$3,0=\mathrm{CSi}\left(\underline{\mathrm{CH}}_{3}\right)_{3}$

S 


\begin{tabular}{|c|c|c|c|c|}
\hline \multirow[t]{9}{*}{$\delta^{13} \mathrm{C}[\mathrm{ppm}]$} & 4,7 & $\mathrm{~N}\left(\mathrm{Si}\left(\underline{\mathrm{CH}}_{3}\right)_{3}\right)_{2}$ & $\mathrm{~d}$ & ${ }^{4} \mathrm{~J}_{\mathrm{CF}}=2,4 \mathrm{~Hz}$ \\
\hline & 22,8 & $\underline{\mathrm{C}} \mathrm{H}$ & $\mathrm{d}$ & ${ }^{2} \mathrm{~J}_{\mathrm{CF}}=20,1 \mathrm{~Hz}$ \\
\hline & 29,6 & $\mathrm{C}\left(\underline{\mathrm{CH}}_{3}\right)_{3}$ & $\mathrm{~s}$ & \\
\hline & 31,1 & $\mathrm{C}\left(\underline{\mathrm{CH}}_{3}\right)_{3}$ & $\mathrm{~s}$ & \\
\hline & 37,3 & $\underline{\mathrm{C}}\left(\mathrm{CH}_{3}\right)_{3}$ & $d$ & ${ }^{4} \mathrm{~J}_{\mathrm{CF}}=0,3 \mathrm{~Hz}$ \\
\hline & 38,5 & $\underline{\mathrm{C}}\left(\mathrm{CH}_{3}\right)_{3}$ & $d$ & ${ }^{4} \mathrm{~J}_{\mathrm{CF}}=0,7 \mathrm{~Hz}$ \\
\hline & 108,0 & $=\underline{\mathrm{C}} \mathrm{H}$ & $\mathrm{d}$ & ${ }^{4} \mathrm{~J}_{\mathrm{CF}}=0,7 \mathrm{~Hz}$ \\
\hline & 155,4 & $\underline{\mathrm{CN}}$ & $d$ & ${ }^{3} \mathrm{~J}_{\mathrm{CF}}=4,3 \mathrm{~Hz}$ \\
\hline & 165,3 & $\underline{\mathrm{CN}}$ & $d$ & ${ }^{3} \mathrm{~J}_{\mathrm{CF}}=1,5 \mathrm{~Hz}$ \\
\hline$\delta{ }^{19} \mathrm{~F}$ [ppm] & 40,5 & $\mathrm{SiE}$ & $d$ & ${ }^{3} \mathrm{~J}_{\mathrm{FH}}=12,4 \mathrm{~Hz}$ \\
\hline \multirow[t]{4}{*}{$\delta^{29} \mathrm{Si}[\mathrm{ppm}]$} & $-13,5$ & $\underline{\mathrm{SiF}}$ & $d$ & ${ }^{1} \mathrm{~J}_{\mathrm{SiF}}=273 \mathrm{~Hz}$ \\
\hline & $-11,8$ & $=\mathrm{CSi}\left(\mathrm{CH}_{3}\right)_{3}$ & $\mathrm{~s}$ & \\
\hline & 1,5 & $\mathrm{CSi}\left(\mathrm{CH}_{3}\right)_{3}$ & $d$ & ${ }^{3} \mathrm{~J}_{\mathrm{SiF}}=5,8 \mathrm{~Hz}$ \\
\hline & 5,3 & $\mathrm{~N}\left(\underline{\mathrm{Si}}\left(\mathrm{CH}_{3}\right)_{3}\right)_{2}$ & $d$ & ${ }^{3} \mathrm{~J}_{\mathrm{SiF}}=4,4 \mathrm{~Hz}$ \\
\hline
\end{tabular}


NMR ( $\left.\mathrm{CDCl}_{3}\right)$ :

Isomer B:

$\delta^{1} \mathrm{H}[\mathrm{ppm}]$

$\begin{array}{ll}0,02 & =\mathrm{CSi}\left(\mathrm{CH}_{3}\right)_{3} \\ 0,21 & \mathrm{~N}\left(\mathrm{Si}\left(\mathrm{CH}_{3}\right)_{3}\right)_{2} \\ 0,27 & \mathrm{CSi}\left(\mathrm{CH}_{3}\right)_{3} \\ 1,13 & \mathrm{C}\left(\mathrm{C}_{3}\right)_{3} \\ 1,14 & \mathrm{C}\left(\underline{\mathrm{C}}_{3}\right)_{3} \\ 1,43 & \mathrm{C} \underline{\mathrm{H}} \\ 5,50 & =\mathrm{C} \underline{\mathrm{H}}\end{array}$

d

d

$$
{ }^{7} \mathrm{~J}_{\mathrm{HF}}=0,7 \mathrm{~Hz}
$$

$9 \mathrm{H}$

d

${ }^{5} \mathrm{~J}_{\mathrm{HF}}=1,2 \mathrm{~Hz}$

$18 \mathrm{H}$

d

${ }^{5} \mathrm{~J}_{\mathrm{HF}}=1,1 \mathrm{~Hz}$

$9 \mathrm{H}$

$\mathrm{S}$

$9 \mathrm{H}$

$\mathrm{s}$

$9 \mathrm{H}$

d $\quad{ }^{3} \mathrm{~J}_{\mathrm{HF}}=16,5 \mathrm{~Hz}$

$1 \mathrm{H}$

d

${ }^{5} \mathrm{~J}_{\mathrm{HF}}=0,4 \mathrm{~Hz}$

$1 \mathrm{H}$

\begin{tabular}{|c|c|c|c|c|}
\hline \multirow[t]{11}{*}{$\delta{ }^{13} \mathrm{C}[\mathrm{ppm}]$} & 0,8 & $\mathrm{CSi}\left(\underline{\mathrm{CH}}_{3}\right)_{3}$ & $d$ & ${ }^{4} J_{C F}=2,8 \mathrm{~Hz}$ \\
\hline & 2,8 & $=\mathrm{CSi}\left(\underline{\mathrm{CH}}_{3}\right)_{3}$ & $d$ & ${ }^{6} \mathrm{~J}_{\mathrm{CF}}=1,7 \mathrm{~Hz}$ \\
\hline & 5,5 & $\mathrm{~N}\left(\mathrm{Si}\left(\underline{\mathrm{CH}}_{3}\right)_{3}\right)_{2}$ & $d$ & ${ }^{4} \mathrm{~J}_{\mathrm{CF}}=2,3 \mathrm{~Hz}$ \\
\hline & 21,7 & $\underline{\mathrm{C}} \mathrm{H}$ & $d$ & ${ }^{2} \mathrm{~J}_{\mathrm{CF}}=21,4 \mathrm{~Hz}$ \\
\hline & 29,2 & $\mathrm{C}\left(\underline{\mathrm{CH}}_{3}\right)_{3}$ & $\mathrm{~s}$ & \\
\hline & 31,4 & $\mathrm{C}\left(\underline{\mathrm{CH}}_{3}\right)_{3}$ & $\mathrm{~s}$ & \\
\hline & 37,6 & $\underline{\mathrm{C}}\left(\mathrm{CH}_{3}\right)_{3}$ & $\mathrm{~s}$ & \\
\hline & 39,6 & $\underline{\mathrm{C}}\left(\mathrm{CH}_{3}\right)_{3}$ & $d$ & ${ }^{4} \mathrm{~J}_{\mathrm{CF}}=2,0 \mathrm{~Hz}$ \\
\hline & 122,8 & $=\underline{\mathrm{CH}}$ & $\mathrm{s}$ & \\
\hline & 154,2 & $\underline{\mathrm{CN}}$ & $d$ & ${ }^{3} \mathrm{~J}_{\mathrm{CF}}=3,1 \mathrm{~Hz}$ \\
\hline & 164,6 & $\underline{\mathrm{C} N}$ & $d$ & ${ }^{3} \mathrm{~J}_{\mathrm{CF}}=1,8 \mathrm{~Hz}$ \\
\hline$\delta^{19} \mathrm{~F}$ [ppm] & 52,9 & $\mathrm{SiF}$ & $d$ & ${ }^{3} J_{F H}=16,5 \mathrm{~Hz}$ \\
\hline \multirow[t]{4}{*}{$\delta^{29} \mathrm{Si}$ [ppm] } & $-13,9$ & $\underline{\text { SiF }}$ & $d$ & ${ }^{1} \mathrm{~J}_{\mathrm{SiF}}=274 \mathrm{~Hz}$ \\
\hline & $-9,4$ & $=\mathrm{CS} \underline{\mathrm{i}}\left(\mathrm{CH}_{3}\right)_{3}$ & $\mathrm{~s}$ & \\
\hline & 0,5 & $\mathrm{CSi}\left(\mathrm{CH}_{3}\right)_{3}$ & $d$ & ${ }^{3} \mathrm{~J}_{\mathrm{SiF}}=5,3 \mathrm{~Hz}$ \\
\hline & 5,1 & $\mathrm{~N}\left(\underline{\mathrm{Si}}\left(\mathrm{CH}_{3}\right)_{3}\right)_{2}$ & $d$ & ${ }^{3} \mathrm{~J}_{\mathrm{SiF}}=4,6 \mathrm{~Hz}$ \\
\hline
\end{tabular}


Verbindung 32: $\quad$-(5-tert-Butyl-3-fluor-3,4-dihydro-2-(3,3-dimethyl-1(trimethylsilyl)but-1-en-2-yl)-4-(trimethylsilyl)-2H-1,2,3diazasilol-3-yl)-2-methyl-N-(trimethylsilyl)propan-2-amin<smiles>CC(C)(C)C1=NN(/C(=C\[SiH3])C(C)(C)C)[Si](F)(N([SiH3])[SiH3])C1[SiH3]</smiles>

Summenformel: $\quad \mathrm{C}_{25} \mathrm{H}_{56} \mathrm{FN}_{3} \mathrm{Si}_{4}$

Molare Masse: $\quad 530,07 \mathrm{~g} / \mathrm{mol}$

MS (EI)

$529 \mathrm{~g} / \mathrm{mol}$
$514 \mathrm{~g} / \mathrm{mol}$
$472 \mathrm{~g} / \mathrm{mol}$
$458 \mathrm{~g} / \mathrm{mol}$
$73 \mathrm{~g} / \mathrm{mol}$

$\begin{array}{ll}27 \% & {[\mathrm{M}]^{+}} \\ 38 \% & {[\mathrm{M}-\mathrm{Me}]^{+}} \\ 79 \% & {[\mathrm{M}-\mathrm{tBu}]^{+}} \\ 98 \% & {[\mathrm{M}-\mathrm{N} t \mathrm{Bu}]^{+}} \\ 100 \% & {\left[\mathrm{SiMe}_{3}\right]^{+}}\end{array}$

Siedepunkt: $\quad 140{ }^{\circ} \mathrm{C} / 0,01 \mathrm{mbar}$

Ausbeute:

$67 \%$

$\operatorname{NMR}\left(\mathrm{C}_{6} \mathrm{D}_{6}\right)$ :

$\begin{array}{clllll}\delta{ }^{1} \mathbf{H}[\mathbf{p p m}] & 0,25 & \mathrm{CSi}\left(\mathrm{CH}_{3}\right)_{3} & \mathrm{~d} & { }^{5} \mathrm{~J}_{\mathrm{HF}}=1,3 \mathrm{~Hz} & 9 \mathrm{H} \\ & 0,33 & =\mathrm{CSi}\left(\mathrm{C}_{3}\right)_{3} & \mathrm{~s} & & 9 \mathrm{H} \\ 0,38 & \mathrm{NSi}\left(\mathrm{CH}_{3}\right)_{3} & \mathrm{~d} & { }^{5} \mathrm{~J}_{\mathrm{HF}}=2,6 \mathrm{~Hz} & 9 \mathrm{H} \\ 1,22 & \mathrm{CC}\left(\mathrm{CH}_{3}\right)_{3} & \mathrm{~s} & & 9 \mathrm{H} \\ 1,26 & \mathrm{CC}\left(\mathrm{CH}_{3}\right)_{3} & \mathrm{~s} & & 9 \mathrm{H} \\ 1,37 & \mathrm{C} \underline{\mathrm{H}} & \mathrm{d} & { }^{3} \mathrm{~J}_{\mathrm{HF}}=10,3 \mathrm{~Hz} & 1 \mathrm{H} \\ 1,48 & \mathrm{NC}\left(\underline{\mathrm{H}}_{3}\right)_{3} & \mathrm{~s} & & 9 \mathrm{H} \\ 5,42 & =\mathrm{CH} & \mathrm{d} & { }^{5} \mathrm{~J}_{\mathrm{HF}}=1,1 \mathrm{~Hz} & 1 \mathrm{H}\end{array}$




$\begin{array}{rrlll}\delta{ }^{13} \mathrm{C} \text { [ppm] } & 2,3 & \mathrm{CSi}\left(\underline{\mathrm{C}} \mathrm{H}_{3}\right)_{3} & \mathrm{~d} & { }^{4} \mathrm{~J}_{\mathrm{CF}}=2,5 \mathrm{~Hz} \\ 3,4 & =\mathrm{CSi}\left(\underline{\mathrm{C}} \mathrm{H}_{3}\right)_{3} & \mathrm{~s} & \\ 6,7 & \mathrm{NSi}\left(\underline{\mathrm{C}} \mathrm{H}_{3}\right)_{3} & \mathrm{~d} & { }^{4} \mathrm{~J}_{\mathrm{CF}}=5,4 \mathrm{~Hz} \\ 25,3 & \underline{\mathrm{CH}} & \mathrm{d} & { }^{2} \mathrm{~J}_{\mathrm{CF}}=19,8 \mathrm{~Hz} \\ 30,1 & \mathrm{CC}\left(\underline{\mathrm{CH}}_{3}\right)_{3} & \mathrm{~s} & \\ 31,5 & \mathrm{CC}\left(\underline{\mathrm{CH}}_{3}\right)_{3} & \mathrm{~s} & \\ 34,0 & \mathrm{NC}\left(\underline{\mathrm{CH}}_{3}\right)_{3} & \mathrm{~d} & { }^{4} \mathrm{~J}_{\mathrm{CF}}=1,3 \mathrm{~Hz} \\ 37,3 & \underline{\mathrm{C}}\left(\mathrm{CH}_{3}\right)_{3} & \mathrm{~d} & { }^{4} \mathrm{~J}_{\mathrm{CF}}=0,4 \mathrm{~Hz} \\ 38,9 & \underline{\mathrm{C}}\left(\mathrm{CH}_{3}\right)_{3} & \mathrm{~d} & { }^{4} \mathrm{~J}_{\mathrm{CF}}=0,5 \mathrm{~Hz} \\ 54,5 & \mathrm{~N} \underline{\mathrm{C}}\left(\mathrm{CH}_{3}\right)_{3} & \mathrm{~d} & { }^{3} \mathrm{~J}_{\mathrm{CF}}=1,2 \mathrm{~Hz} \\ 106,2 & =\underline{\mathrm{C}} \mathrm{H} & \mathrm{s} & \\ 156,3 & \underline{\mathrm{CN}} & \mathrm{d} & { }^{3} \mathrm{~J}_{\mathrm{CF}}=5,1 \mathrm{~Hz} \\ 166,2 & \underline{\mathrm{CN}} & \mathrm{d} & { }^{3} \mathrm{~J}_{\mathrm{CF}}=1,2 \mathrm{~Hz}\end{array}$

$\delta^{19} \mathrm{~F}[\mathrm{ppm}] \quad 35,5 \quad$ SiFN $\quad \mathrm{s}$

$\begin{array}{rrlll}\delta{ }^{29} \mathrm{Si}[\mathrm{ppm}] & -11,9 & \underline{\mathrm{SiFN}} & \mathrm{d} & { }^{1} \mathrm{~J}_{\mathrm{SiF}}=264 \mathrm{~Hz} \\ -11,8 & =\mathrm{CSi}\left(\mathrm{CH}_{3}\right)_{3} & \mathrm{~s} & \\ 1,7 & \mathrm{CS}\left(\mathrm{CH}_{3}\right)_{3} & \mathrm{~d} & { }^{3} \mathrm{~J}_{\mathrm{SiF}}=6,4 \mathrm{~Hz} \\ 5,3 & \mathrm{NS}\left(\mathrm{CH}_{3}\right)_{3} & \mathrm{~d} & { }^{3} \mathrm{~J}_{\mathrm{SiF}}=3,9 \mathrm{~Hz}\end{array}$


Verbindung 33: 1,9-Bis(3,3-dimethylbut-1-en-2-yl)-3,7-tert-butyl-1,2,8,9tetraaza-5-sila-spiro[4.4]nona-2,7-dien<smiles>C=C(N1N=C(C(C)(C)C)C[Si]12CC(C(C)(C)C)=NN2C(=C)C(C)(C)C)C(C)(C)C</smiles>

Summenformel: $\quad \mathrm{C}_{24} \mathrm{H}_{44} \mathrm{~N}_{4} \mathrm{Si}$

Molare Masse: $\quad 416,72 \mathrm{~g} / \mathrm{mol}$

MS (EI)

$416 \mathrm{~g} / \mathrm{mol}$

$10 \% \quad[\mathrm{M}]^{+}$

$401 \mathrm{~g} / \mathrm{mol}$

$2 \% \quad[\mathrm{M}-\mathrm{Me}]^{+}$

$359 \mathrm{~g} / \mathrm{mol}$

$100 \% \quad[\mathrm{M}-\mathrm{tBu}]^{+}$

Siedepunkt: $\quad 142{ }^{\circ} \mathrm{C} / 0,04 \mathrm{mbar}$

Ausbeute: $\quad 35 \%$

NMR ( $\left.\mathrm{CDCl}_{3}\right)$ :

$\delta^{1} \mathrm{H}[\mathrm{ppm}]$

$\begin{array}{ll}1,13 & \mathrm{C}\left(\mathrm{C}_{3}\right)_{3} \\ 1,19 & \mathrm{C}\left(\mathrm{CH}_{3}\right)_{3} \\ 1,57 & \mathrm{C} \underline{H}_{2} \mathrm{a} \\ 1,78 & \mathrm{C}_{2} \mathrm{~b} \\ 3,84 & =\underline{\mathrm{CH}}_{2} \mathrm{a} \\ 4,10 & =\underline{\mathrm{CH}}_{2} \mathrm{~b}\end{array}$

$S$

$18 \mathrm{H}$

$\mathrm{s}$

$18 \mathrm{H}$

d $\quad 2 \mathrm{~J}_{\mathrm{H}_{\mathrm{a}} \mathrm{H}_{\mathrm{b}}}=19,6 \mathrm{~Hz} \quad 2 \mathrm{H}$

d $\quad 2 \mathrm{~J}_{\mathrm{H}_{\mathrm{b}} \mathrm{H}_{\mathrm{a}}}=19,6 \mathrm{~Hz} \quad 2 \mathrm{H}$

d $\quad{ }^{2} \mathrm{~J}_{\mathrm{H}_{\mathrm{a}} \mathrm{H}_{\mathrm{b}}}=0,7 \mathrm{~Hz} \quad 2 \mathrm{H}$

$\delta{ }^{13} \mathrm{C}[\mathrm{ppm}] \quad 10,8 \quad \underline{\mathrm{CH}}_{2} \quad \mathrm{~s}$

28,6 $\mathrm{C}\left(\underline{\mathrm{CH}}_{3}\right)_{3} \quad \mathrm{~s}$

$29,8 \quad \mathrm{C}\left(\underline{\mathrm{CH}}_{3}\right)_{3} \quad \mathrm{~s}$

$36,7 \quad \underline{\mathrm{C}}\left(\mathrm{CH}_{3}\right)_{3} \quad \mathrm{~s}$

36,9 $\underline{\mathrm{C}}\left(\mathrm{CH}_{3}\right)_{3} \quad \mathrm{~s}$ 


$\begin{array}{rrll}\delta{ }^{13} \mathrm{C}[\mathrm{ppm}] & 89,7 & =\underline{\mathrm{C}} \mathrm{H}_{2} & \mathrm{~s} \\ 157,3 & \underline{\mathrm{CN}} & \mathrm{s} \\ 158,6 & \underline{\mathrm{CN}} & \mathrm{s}\end{array}$

$\delta^{29} \mathrm{Si}[\mathrm{ppm}] \quad 9,8 \quad \mathrm{NSiC} \quad \mathrm{s}$ 
Verbindung 34: 1,9-Bis(3,3-dimethyl-1-(trimethylsilyl)but-1-en-2-yl)-3,7-tertbutyl-4,6-(trimethylsilyl)-1,2,8,9-tetraaza-5-sila-spiro[4.4]nona2,7-dien<smiles>C[SiH2]/C=C(/N1N=C(C(C)(C)C)C([SiH3])[Si]12C([SiH3])C(C)=NN2C(C)(C)C)C(C)(C)C</smiles>

Summenformel: $\quad \mathrm{C}_{36} \mathrm{H}_{76} \mathrm{~N}_{4} \mathrm{Si}_{5}$

Molare Masse: $\quad 705,44 \mathrm{~g} / \mathrm{mol}$

MS (EI)

$704 \mathrm{~g} / \mathrm{mol}$

$100 \% \quad[\mathrm{M}]^{+}$

$689 \mathrm{~g} / \mathrm{mol}$

$7 \% \quad[\mathrm{M}-\mathrm{Me}]^{+}$

$647 \mathrm{~g} / \mathrm{mol}$

$9 \% \quad[\mathrm{M}-\mathrm{tBu}]^{+}$

Siedepunkt: $\quad 210^{\circ} \mathrm{C} / 0,01 \mathrm{mbar}$

Ausbeute: $\quad 33 \%$

$\operatorname{NMR}\left(\mathrm{CDCl}_{3}\right)$ :*

$\delta{ }^{1} \mathrm{H}[\mathrm{ppm}]$

$0,07 \quad \mathrm{CSi}\left(\mathrm{C}_{\mathrm{H}_{3}}\right)_{3}$

S

$9 \mathrm{H}$

$0,21=\mathrm{CSi}\left(\mathrm{C}_{\mathrm{H}_{3}}\right)_{3}$

$9 \mathrm{H}$

$1,19 \quad \mathrm{C}\left(\mathrm{C}_{3}\right)_{3}$

$9 \mathrm{H}$

$1,29 \quad \mathrm{C}\left(\mathrm{CH}_{3}\right)_{3}$

$9 \mathrm{H}$

$2,02 \quad \mathrm{C} \underline{\mathrm{H}}$

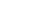

$1 \mathrm{H}$

$4,38=\mathrm{C} \underline{\mathrm{H}}$

$\mathrm{S}$

$1 \mathrm{H}$

$\begin{array}{rrll}\delta{ }^{13} \mathrm{C}[\mathrm{ppm}] & 3,1 & \mathrm{CSi}\left(\underline{\mathrm{C}} \mathrm{H}_{3}\right)_{3} & \mathrm{~s} \\ 3,3 & =\mathrm{CSi}\left(\underline{\mathrm{CH}} \mathrm{H}_{3}\right)_{3} & \mathrm{~s} \\ 20,7 & \underline{\mathrm{C}} \mathrm{H} & \mathrm{s} \\ 29,4 & \mathrm{C}\left(\underline{\mathrm{C}} \mathrm{H}_{3}\right)_{3} & \mathrm{~s}\end{array}$




$\begin{array}{rrlr}\delta{ }^{13} \mathrm{C} \text { [ppm] } & 30,9 & \mathrm{C}\left(\mathrm{CH}_{3}\right)_{3} & \mathrm{~s} \\ 38,3 & \underline{\mathrm{C}}\left(\mathrm{CH}_{3}\right)_{3} & \mathrm{~s} \\ 38,5 & \underline{\mathrm{C}}\left(\mathrm{CH}_{3}\right)_{3} & \mathrm{~s} \\ 100,6 & =\underline{\mathrm{C}} \mathrm{H} & \mathrm{s} \\ 154,9 & \underline{\mathrm{CN}} & \mathrm{s} \\ 165,1 & \underline{\mathrm{CN}} & \mathrm{s}\end{array}$

$\delta^{29} \mathrm{Si}[\mathrm{ppm}] \quad-11,8 \quad=\mathrm{CSi}\left(\mathrm{CH}_{3}\right)_{3}$

$$
-3,0 \quad \mathrm{CSi}\left(\mathrm{CH}_{3}\right)_{3}
$$

16,1 NSiC

*: zugeordnet wurde nur das Hauptisomer
S

S

s

$\mathrm{S}$

S

S

s $\quad{ }^{1} \mathrm{~J}_{29 \mathrm{Si}^{13} \mathrm{C}}=69,6 \mathrm{~Hz}$

${ }^{1} \mathrm{~J}_{29 \mathrm{Si}^{13} \mathrm{C}_{3}}=53,2 \mathrm{~Hz}$

S $\quad{ }^{1} \mathrm{~J}_{29} \mathrm{Si}^{13 \mathrm{C}}=44,6 \mathrm{~Hz}$

${ }^{1} \mathrm{~J}_{29 \mathrm{Si}^{13} \mathrm{C}_{3}}=52,6 \mathrm{~Hz}$

s $\quad{ }^{1} \mathrm{~J}_{29} \mathrm{Si}^{13} \mathrm{C}_{2}=59,2 \mathrm{~Hz}$ 
Verbindung 35: 2-(1-(Di-tert-butyl(methoxi)silyl)-3,3-dimethylbutan-2-yliden)1-(3,3-dimethylbutan-2-yliden)hydrazin

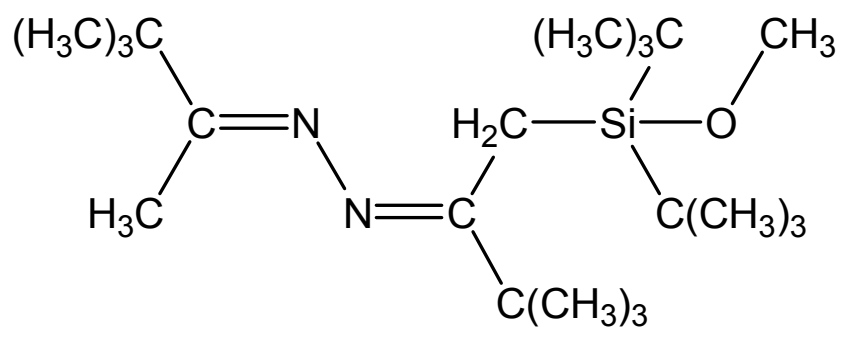

Summenformel: $\quad \mathrm{C}_{21} \mathrm{H}_{44} \mathrm{~N}_{2} \mathrm{OSi}$

Molare Masse: $\quad 368,67 \mathrm{~g} / \mathrm{mol}$

MS (EI)

$\begin{array}{lcl}368 \mathrm{~g} / \mathrm{mol} & 3 \% & {[\mathrm{M}]^{+}} \\ 353 \mathrm{~g} / \mathrm{mol} & 2 \% & {[\mathrm{M}-\mathrm{Me}]^{+}} \\ 311 \mathrm{~g} / \mathrm{mol} & 100 \% & {[\mathrm{M}-\mathrm{tBu}]^{+}}\end{array}$

Siedepunkt: $\quad 74^{\circ} \mathrm{C} / 0,01 \mathrm{mbar}$

Ausbeute: $\quad 71 \%$

$\operatorname{NMR}\left(\mathrm{C}_{6} \mathrm{D}_{6}\right)$ :

\begin{tabular}{|c|c|c|c|c|}
\hline \multirow[t]{6}{*}{$\delta^{1} \mathrm{H}$ [ppm] } & 1,09 & $\mathrm{Si}\left(\mathrm{C}\left(\mathrm{CH}_{3}\right)_{3}\right)_{2}$ & $\mathrm{~s}$ & $18 \mathrm{H}$ \\
\hline & 1,16 & $\mathrm{C}\left(\mathrm{C}_{3}\right)_{3}$ & $\mathrm{~s}$ & $9 \mathrm{H}$ \\
\hline & 1,41 & $\mathrm{C}\left(\mathrm{C}_{3}\right)_{3}$ & $\mathrm{~s}$ & $9 \mathrm{H}$ \\
\hline & 1,91 & $\mathrm{CC}_{3}$ & $\mathrm{~s}$ & $3 \mathrm{H}$ \\
\hline & 2,39 & $\mathrm{C}_{2}$ & $\mathrm{~s}$ & $\mathrm{H}$ \\
\hline & 3,45 & $\mathrm{OCH}_{3}$ & $\mathrm{~s}$ & $3 \mathrm{H}$ \\
\hline \multirow[t]{8}{*}{$\delta{ }^{13} \mathrm{C}$ [ppm] } & 13,6 & $\mathrm{C}_{\mathrm{CH}} \mathrm{H}_{3}$ & $\mathrm{~s}$ & \\
\hline & 14,4 & $\underline{\mathrm{C}}_{2}$ & $s$ & \\
\hline & 22,4 & $\mathrm{Si}\left(\underline{\mathrm{C}}\left(\mathrm{CH}_{3}\right)_{3}\right)_{2}$ & $\mathrm{~s}$ & \\
\hline & 28,0 & $\mathrm{C}\left(\underline{\mathrm{C}}_{3}\right)_{3}$ & $\mathrm{~s}$ & \\
\hline & 28,9 & $\mathrm{Si}\left(\mathrm{C}\left(\mathrm{CH}_{3}\right)_{3}\right)_{2}$ & $\mathrm{~s}$ & \\
\hline & 29,8 & $\mathrm{C}\left(\underline{\mathrm{C}}_{3}\right)_{3}$ & $\mathrm{~s}$ & \\
\hline & 38,9 & $\underline{\mathrm{C}}\left(\mathrm{CH}_{3}\right)_{3}$ & $\mathrm{~s}$ & \\
\hline & 39,5 & $\underline{\mathrm{C}}\left(\mathrm{CH}_{3}\right)_{3}$ & $\mathrm{~s}$ & \\
\hline
\end{tabular}




$\begin{array}{rrll}\delta{ }^{13} \mathrm{C}[\mathrm{ppm}] & 52,6 & \mathrm{O}_{\mathrm{CH}} & \mathrm{s} \\ & 169,4 & \underline{\mathrm{CN}} & \mathrm{s} \\ 173,2 & \underline{\mathrm{CN}} & \mathrm{s}\end{array}$

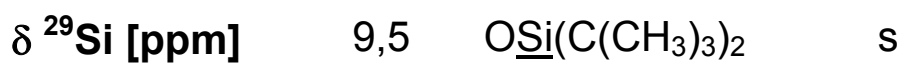


Verbindung 36: 3,5-Di-tert-butyl-3,4-dihydro-3-methyl-2-(3,3-dimethyl-1(trimethylsilyl)but-1-en-2-yl)-4-(trimethylsilyl)-2H-1,2,3diazasilol<smiles>CC(C)(C)C1=NN(/C(=C\[SiH3])C(C)(C)C)[Si](C)(C)C1[SiH3]</smiles>

Summenformel: $\quad \mathrm{C}_{23} \mathrm{H}_{50} \mathrm{~N}_{2} \mathrm{Si}_{3}$

Molare Masse: $\quad 438,91 \mathrm{~g} / \mathrm{mol}$

MS (EI)

$438 \mathrm{~g} / \mathrm{mol}$
$423 \mathrm{~g} / \mathrm{mol}$
$381 \mathrm{~g} / \mathrm{mol}$
$73 \mathrm{~g} / \mathrm{mol}$

$63 \% \quad[\mathrm{M}]^{+}$

$60 \% \quad[\mathrm{M}-\mathrm{Me}]^{+}$

$67 \% \quad[\mathrm{M}-\mathrm{tBu}]^{+}$

$124^{\circ} \mathrm{C} / 0,24 \mathrm{mbar}$

Siedepunkt:

$78 \%$

NMR $\left(\mathrm{CDCl}_{3}\right)$ :

$\delta{ }^{1} \mathrm{H}$ [ppm]

$0,10 \quad \mathrm{CSi}\left(\mathrm{CH}_{3}\right)_{3}$

$0,30=\mathrm{CSi}\left(\mathrm{C}_{3}\right)_{3}$

$9 \mathrm{H}$

$0,50 \quad \mathrm{SiC}_{3}$

$3 \mathrm{H}$

$0,90 \quad \mathrm{SiC}\left(\mathrm{CH}_{3}\right)_{3}$

$9 \mathrm{H}$

$1,23 \quad \mathrm{CC}\left(\mathrm{CH}_{3}\right)_{3}$

$9 \mathrm{H}$

$1,47 \quad \mathrm{CC}\left(\mathrm{CH}_{3}\right)_{3}$

$9 \mathrm{H}$

$1,49 \quad \mathrm{C} \underline{\mathrm{H}}$

$1 \mathrm{H}$

$4,49=\mathrm{C} \underline{\mathrm{H}}$

$\mathrm{s}$

$1 \mathrm{H}$

$\begin{array}{rrll}\delta{ }^{13} \mathrm{C}[\mathrm{ppm}] & -4,8 & \mathrm{Si} \underline{\mathrm{C}} \mathrm{H}_{3} & \mathrm{~s} \\ 2,2 & \mathrm{CSi}\left(\underline{\mathrm{C}} \mathrm{H}_{3}\right)_{3} & \mathrm{~s} \\ 3,6 & =\mathrm{CSi}\left(\underline{\mathrm{CH}} \mathrm{H}_{3}\right)_{3} & \mathrm{~s}\end{array}$




$\begin{array}{rrlr}\delta{ }^{13} \mathrm{C} \text { [ppm] } & 21,0 & \underline{\mathrm{CH}} & \mathrm{s} \\ 21,4 & \mathrm{Si} \underline{\mathrm{C}}(\mathrm{CH})_{3} & \mathrm{~s} \\ 27,2 & \left.\mathrm{CC}(\underline{\mathrm{CH}})_{3}\right)_{3} & \mathrm{~s} \\ 30,5 & \left.\mathrm{CC}(\underline{\mathrm{CH}})_{3}\right)_{3} & \mathrm{~s} \\ 31,5 & \mathrm{SiC}(\underline{\mathrm{C}} \mathrm{H})_{3} & \mathrm{~s} \\ 36,9 & \mathrm{C} \underline{\mathrm{C}}\left(\mathrm{CH}_{3}\right)_{3} & \mathrm{~s} \\ 38,7 & \mathrm{C} \underline{\mathrm{C}}\left(\mathrm{CH}_{3}\right)_{3} & \mathrm{~s} \\ 99,1 & =\underline{\mathrm{C}} \mathrm{H} & \mathrm{s} \\ 158,3 & \underline{\mathrm{CN}} & \mathrm{s} \\ 167,5 & \underline{\mathrm{CN}} & \mathrm{s}\end{array}$

$\begin{array}{rrll}\delta{ }^{29} \mathrm{Si} \text { [ppm] } & -12,0 & =\mathrm{CSi}\left(\mathrm{CH}_{3}\right)_{3} & \mathrm{~s} \\ -0,6 & \mathrm{CSi}\left(\mathrm{CH}_{3}\right)_{3} & \mathrm{~s} \\ 29,8 & \underline{\operatorname{SiC}(\mathrm{CH})_{3}} & \mathrm{~s}\end{array}$


Verbindung 37: 3,5-Di-tert-butyl-3-butyl-3,4-dihydro-2-(3,3-dimethyl-1(trimethylsilyl)but-1-en-2-yl)-4-(trimethylsilyl)-2H-1,2,3diazasilol<smiles>CC(C)(C)C1=NN(/C(=C\[SiH3])C(C)(C)C)[Si](C(C)(C)C)(C(C)(C)C)C1[SiH3]</smiles>

Summenformel: $\quad \mathrm{C}_{26} \mathrm{H}_{56} \mathrm{~N}_{2} \mathrm{Si}_{3}$

Molare Masse: $\quad 480,99 \mathrm{~g} / \mathrm{mol}$

MS (EI)

$480 \mathrm{~g} / \mathrm{mol}$
$465 \mathrm{~g} / \mathrm{mol}$
$423 \mathrm{~g} / \mathrm{mol}$
$367 \mathrm{~g} / \mathrm{mol}$

$63 \% \quad[\mathrm{M}]^{+}$

$39 \% \quad[\mathrm{M}-\mathrm{Me}]^{+}$

$70 \% \quad[\mathrm{M}-\mathrm{tBu}]^{+}$

$100 \% \quad\left[\mathrm{M}-\mathrm{tBu}-\mathrm{C}_{4} \mathrm{H}_{8}\right]^{+}$

Siedepunkt: $\quad 133^{\circ} \mathrm{C} / 0,01 \mathrm{mbar}$

Ausbeute: $\quad 73 \%$

$\operatorname{NMR}\left(\mathrm{CDCl}_{3}\right)$ :

$\delta{ }^{1} \mathrm{H}[\mathrm{ppm}]$

$\begin{array}{rlcc}0,13 & \mathrm{CSi}\left(\mathrm{CH}_{3}\right)_{3} & \text { s } & 9 \mathrm{H} \\ 0,18 & =\mathrm{CSi}\left(\mathrm{CH}_{3}\right)_{3} & \mathrm{~s} & 9 \mathrm{H} \\ 0,90 & \mathrm{SiC}\left(\mathrm{CH}_{3}\right)_{3} & \mathrm{~s} & 9 \mathrm{H} \\ 1,18 & \mathrm{CC}\left(\mathrm{C}_{3}\right)_{3} & \mathrm{~s} & 9 \mathrm{H} \\ 1,26 & \mathrm{CC}\left(\underline{\mathrm{C}}_{3}\right)_{3} & \mathrm{~s} & 9 \mathrm{H} \\ 1,45 & \mathrm{C} \underline{\mathrm{H}} & \mathrm{s} & 1 \mathrm{H} \\ 4,37 & =\mathrm{C} \underline{\mathrm{H}} & \mathrm{s} & 1 \mathrm{H} \\ 0,9-1,5 & \mathrm{C}_{4} \underline{\mathrm{H}}_{9} & \mathrm{~m} & 9 \mathrm{H}\end{array}$

$\begin{array}{rrll}\delta{ }^{13} \mathrm{C} \text { [ppm] } & 2,3 & \mathrm{CSi}\left(\underline{\mathrm{C}} \mathrm{H}_{3}\right)_{3} & \mathrm{~s} \\ 3,1 & =\mathrm{CSi}\left(\underline{\mathrm{C}} \mathrm{H}_{3}\right)_{3} & \mathrm{~s} \\ 10,6 & n-\mathrm{Bu}\left(\underline{\mathrm{C}} \mathrm{H}_{2}\right) & \mathrm{s}\end{array}$




$\begin{array}{rrlr}\delta{ }^{13} \mathrm{C}[\mathrm{ppm}] & 13,7 & n-\mathrm{Bu}\left(\underline{\mathrm{C}} \mathrm{H}_{3}\right) & \mathrm{s} \\ 20,5 & \underline{\mathrm{C}} \mathrm{H} & \mathrm{s} \\ 21,8 & \mathrm{Si} \underline{\mathrm{C}}(\mathrm{CH})_{3} & \mathrm{~s} \\ 25,7 & n-\mathrm{Bu}\left(\underline{\mathrm{C}} \mathrm{H}_{2}\right) \mathrm{b} & \mathrm{s} \\ 27,1 & n-\mathrm{Bu}\left(\underline{\mathrm{C}} \mathrm{H}_{2}\right) \mathrm{c} & \mathrm{s} \\ 27,5 & \mathrm{SiC}(\underline{\mathrm{C}})_{3} & \mathrm{~s} \\ 30,0 & \mathrm{CC}\left(\underline{\mathrm{C}} \mathrm{H}_{3}\right)_{3} & \mathrm{~s} \\ 31,2 & \mathrm{CC}\left(\underline{\mathrm{C}} \mathrm{H}_{3}\right)_{3} & \mathrm{~s} \\ 37,0 & \underline{\mathrm{C}}\left(\mathrm{CH}_{3}\right)_{3} & \mathrm{~s} \\ 38,3 & \underline{\mathrm{C}}\left(\mathrm{CH}_{3}\right)_{3} & \mathrm{~s} \\ 98,0 & =\underline{\mathrm{C}} \mathrm{H} & \mathrm{s} \\ 158,1 & \underline{\mathrm{CN}} & \mathrm{s} \\ 167,4 & \underline{\mathrm{CN}} & \mathrm{s}\end{array}$

$\delta^{29} \mathrm{Si}[\mathrm{ppm}] \quad-12,4 \quad=\mathrm{CSi}\left(\mathrm{CH}_{3}\right)_{3} \quad \mathrm{~s}$

$-0,7 \quad \mathrm{CSi}\left(\mathrm{CH}_{3}\right)_{3} \quad \mathrm{~s}$

29,7 n-BuSiC $(\mathrm{CH})_{3} \quad \mathrm{~s}$ 
Verbindung 38: 3,5-Di-tert-butyl-3,4-dihydro-2-(3,3-dimethyl-1-(trimethylsilyl)but-1-en-2-yl)-3-phenyl-4-(trimethylsilyl)-2H-1,2,3diazasilol

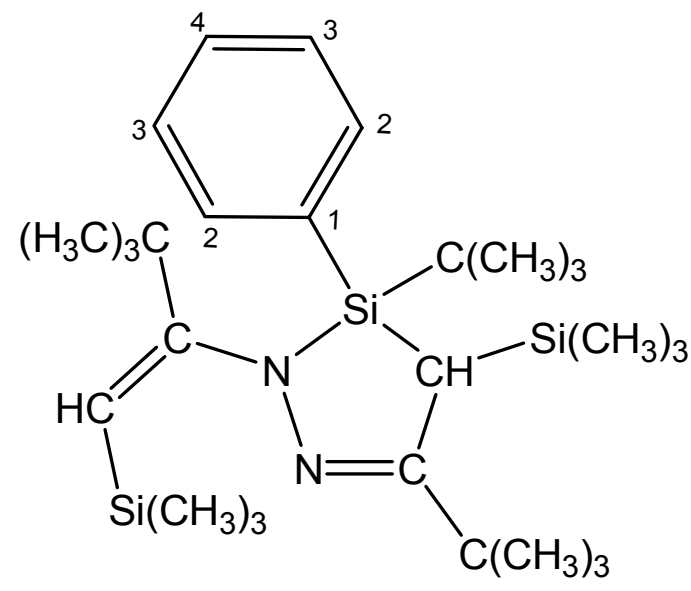

Summenformel: $\quad \mathrm{C}_{28} \mathrm{H}_{52} \mathrm{~N}_{2} \mathrm{Si}_{3}$

Molare Masse: $\quad 500,98 \mathrm{~g} / \mathrm{mol}$

MS (EI)

$500 \mathrm{~g} / \mathrm{mol}$

$94 \% \quad[\mathrm{M}]^{+}$

$423 \mathrm{~g} / \mathrm{mol}$

$28 \% \quad[\mathrm{M}-\mathrm{Me}]^{+}$

$443 \mathrm{~g} / \mathrm{mol}$

$59 \% \quad[\mathrm{M}-\mathrm{tBu}]^{+}$

$387 \mathrm{~g} / \mathrm{mol}$

$100 \% \quad\left[\mathrm{M}-\mathrm{tBu}-\mathrm{C}_{4} \mathrm{H}_{8}\right]^{+}$

Siedepunkt: $\quad 162{ }^{\circ} \mathrm{C} / 0,008 \mathrm{mbar}$

Ausbeute: $\quad 76 \%$

NMR ( $\left.\mathrm{CDCl}_{3}\right)$ :

$\begin{array}{rrlll}\delta{ }^{1} \mathbf{H}[\mathrm{ppm}] & 0,16 & \mathrm{CSi}\left(\mathrm{CH}_{3}\right)_{3} & \mathrm{~s} & 9 \mathrm{H} \\ 0,17 & =\mathrm{CSi}\left(\mathrm{C}_{3}\right)_{3} & \mathrm{~s} & 9 \mathrm{H} \\ 1,22 & \mathrm{SiC}\left(\mathrm{C}_{\mathrm{H}_{3}}\right)_{3} & \mathrm{~s} & 9 \mathrm{H} \\ 1,25 & \mathrm{CC}\left(\underline{\mathrm{CH}}_{3}\right)_{3} & \mathrm{~s} & 9 \mathrm{H} \\ 1,39 & \mathrm{CC}\left(\mathrm{CH}_{3}\right)_{3} & \mathrm{~s} & 9 \mathrm{H} \\ 1,70 & \mathrm{CH} & \mathrm{H} & 1 \mathrm{H} \\ 3,85 & =\mathrm{C} \underline{\mathrm{H}} & \mathrm{s} & 1 \mathrm{H} \\ 7,3-7,7 & \mathrm{C} & \underline{\mathrm{H}}_{5} & \mathrm{~m} & 5 \mathrm{H}\end{array}$




$\begin{array}{rrlr}\delta{ }^{13} \mathrm{C} \text { [ppm] } & 1,8 & \mathrm{CSi}\left(\underline{\mathrm{C}} \mathrm{H}_{3}\right)_{3} & \mathrm{~s} \\ 3,0 & =\mathrm{CSi}\left(\underline{\mathrm{C}} \mathrm{H}_{3}\right)_{3} & \mathrm{~s} \\ 21,3 & \underline{\mathrm{C}} \mathrm{H} & \mathrm{s} \\ 21,6 & \mathrm{Si} \underline{\mathrm{C}}(\mathrm{CH})_{3} & \mathrm{~s} \\ 28,8 & \mathrm{SiC}(\underline{\mathrm{C}})_{3} & \mathrm{~s} \\ 30,2 & \mathrm{CC}\left(\underline{\mathrm{C}} \mathrm{H}_{3}\right)_{3} & \mathrm{~s} \\ 30,9 & \mathrm{CC}\left(\underline{\mathrm{C}} \mathrm{H}_{3}\right)_{3} & \mathrm{~s} \\ 37,1 & \mathrm{C} \underline{\mathrm{C}}\left(\mathrm{CH}_{3}\right)_{3} & \mathrm{~s} \\ 38,0 & \mathrm{C} \underline{\mathrm{C}}(\mathrm{CH})_{3} & \mathrm{~s} \\ 99,9 & =\underline{\mathrm{C}} \mathrm{H} & \mathrm{s} \\ 127,4 & \mathrm{Ph}(\underline{\mathrm{C}} 3 / 2) & \mathrm{s} \\ 129,9 & \mathrm{Ph}(\underline{\mathrm{C}} 4) & \mathrm{s} \\ 131,8 & \mathrm{Ph}(\underline{\mathrm{C}} 1) & \mathrm{s} \\ 136,9 & \mathrm{Ph}(\underline{\mathrm{C}} 2 / 3) & \mathrm{s} \\ 158,6 & \underline{\mathrm{CN}} & \mathrm{s} \\ 165,5 & \underline{\mathrm{CN}} & \mathrm{s}\end{array}$

$\delta^{29} \mathrm{Si}[\mathrm{ppm}] \quad-12,0 \quad=\mathrm{CSi}\left(\mathrm{CH}_{3}\right)_{3} \quad \mathrm{~s}$

$-0,5 \quad \mathrm{CSi}\left(\mathrm{CH}_{3}\right)_{3} \quad \mathrm{~s}$

19,9 $\mathrm{PhSiC}(\mathrm{CH})_{3} \quad$ s 
Verbindung 39: $\quad$ N-(5-tert-Butyl-3-fluor-3,4-dihydro-2-(3,3-dimethylbut-1-en-2yl)-4-(trimethylsilyl)-2H-1,2,3-diazasilol-3-yl)-2-methyl- $N$ (trimethylsilyl)propan-2-amin<smiles>C=C(N1N=C(C(C)(C)C)C([SiH2]C(C)(C)C)[Si]1(F)N([SiH3])C(C)(C)C)C(C)(C)C</smiles>

Summenformel: $\quad \mathrm{C}_{22} \mathrm{H}_{48} \mathrm{FN}_{3} \mathrm{Si}_{3}$

Molare Masse: $\quad 457,89 \mathrm{~g} / \mathrm{mol}$

MS (EI)

$457 \mathrm{~g} / \mathrm{mol}$
$442 \mathrm{~g} / \mathrm{mol}$
$400 \mathrm{~g} / \mathrm{mol}$
$386 \mathrm{~g} / \mathrm{mol}$

$\begin{array}{ll}23 \% & {[\mathrm{M}]^{+}} \\ 21 \% & {[\mathrm{M}-\mathrm{Me}]^{+}} \\ 83 \% & {[\mathrm{M}-\mathrm{BBu}]^{+}} \\ 100 \% & {[\mathrm{M}-\mathrm{N} t \mathrm{Bu}]^{+}}\end{array}$

Siedepunkt: $\quad 126{ }^{\circ} \mathrm{C} / 0,03 \mathrm{mbar}$

Ausbeute: $\quad 68 \%$

\section{$\operatorname{NMR}\left(\mathrm{C}_{6} \mathrm{D}_{6}\right)$ : $^{*}$}

\begin{tabular}{|c|c|c|c|c|}
\hline$\delta^{1} \mathrm{H}[\mathrm{ppm}]$ & 0,24 & $\mathrm{NSi}\left(\mathrm{CH}_{3}\right)_{3}$ & $d$ & ${ }^{5} \mathrm{~J}_{\mathrm{HF}}=1,4 \mathrm{~Hz}$ \\
\hline & 0,36 & $\mathrm{CSi}\left(\mathrm{C}_{\mathrm{H}_{3}}\right)_{3}$ & $d$ & ${ }^{5} \mathrm{~J}_{\mathrm{HF}}=2,7 \mathrm{~Hz}$ \\
\hline & 1,22 & $\mathrm{CC}\left(\mathrm{C}_{3}\right)_{3}$ & $s$ & \\
\hline & 1,23 & $\mathrm{NC}\left(\mathrm{C}_{3}\right)_{3}$ & $\mathrm{~s}$ & \\
\hline & 1,40 & $\mathrm{C} \underline{\mathrm{H}}$ & $d$ & ${ }^{3} \mathrm{~J}_{\mathrm{HF}}=9,5 \mathrm{~Hz}$ \\
\hline & 1,42 & $\mathrm{CC}\left(\mathrm{C}_{3}\right)_{3}$ & $\mathrm{~s}$ & \\
\hline & 4,44 & $=\mathrm{CH}_{2} \mathrm{a}$ & $\underline{A B X}$ & ${ }^{2} \mathrm{~J}_{\mathrm{H}_{\mathrm{a}} \mathrm{H}_{\mathrm{b}}}=0,4 \mathrm{~Hz}$ \\
\hline & & & & ${ }^{5} \mathrm{~J}_{\mathrm{H}_{\mathrm{a}} \mathrm{F}}$ nicht aufgelöst \\
\hline & 4,86 & $=\underline{C H}_{2} \mathrm{~b}$ & $A \underline{B} X$ & ${ }^{2} \mathrm{~J}_{\mathrm{H}_{\mathrm{b}} \mathrm{H}_{\mathrm{a}}}=0,4 \mathrm{~Hz}$ \\
\hline & & & & ${ }^{5} \mathrm{~J}_{\mathrm{H}_{\mathrm{b}} \mathrm{F}}$ nicht aufgelöst \\
\hline
\end{tabular}




$\begin{array}{rrlll}\delta{ }^{13} \mathrm{C} \text { [ppm] } & 2,1 & \mathrm{CSi}\left(\underline{\mathrm{C}} \mathrm{H}_{3}\right)_{3} & \mathrm{~d} & { }^{4} \mathrm{~J}_{\mathrm{CF}}=2,6 \mathrm{~Hz} \\ 6,6 & \mathrm{NSi}\left(\underline{\mathrm{C}} \mathrm{H}_{3}\right)_{3} & \mathrm{~d} & { }^{4} \mathrm{~J}_{\mathrm{CF}}=5,6 \mathrm{~Hz} \\ 25,0 & \underline{\mathrm{CH}} & \mathrm{d} & { }^{2} \mathrm{~J}_{\mathrm{CF}}=19,5 \mathrm{~Hz} \\ 30,2 & \mathrm{CC}\left(\underline{\mathrm{C}} \mathrm{H}_{3}\right)_{3} & \mathrm{~s} & \\ 30,4 & \mathrm{CC}\left(\underline{\mathrm{C}} \mathrm{H}_{3}\right)_{3} & \mathrm{~s} & \\ 33,7 & \mathrm{NC}\left(\underline{\mathrm{CH}}_{3}\right)_{3} & \mathrm{~d} & { }^{4} \mathrm{~J}_{\mathrm{CF}}=1,0 \mathrm{~Hz} \\ 37,0 & \underline{\mathrm{C}}\left(\mathrm{CH}_{3}\right)_{3} & \mathrm{~d} & { }^{4} \mathrm{~J}_{\mathrm{CF}}=0,4 \mathrm{~Hz} \\ 37,2 & \underline{\mathrm{C}}\left(\mathrm{CH}_{3}\right)_{3} & \mathrm{~d} & { }^{4} \mathrm{~J}_{\mathrm{CF}}=0,4 \mathrm{~Hz} \\ 54,3 & \mathrm{~N} \underline{\mathrm{C}}\left(\mathrm{CH}_{3}\right)_{3} & \mathrm{~d} & { }^{3} \mathrm{~J}_{\mathrm{CF}}=1,4 \mathrm{~Hz} \\ 90,3 & =\underline{\mathrm{C}} \mathrm{H}_{2} & \mathrm{~d} & { }^{4} \mathrm{~J}_{\mathrm{CF}}=1,2 \mathrm{~Hz} \\ 156,9 & \underline{\mathrm{CN}} & \mathrm{d} & { }^{3} \mathrm{~J}_{\mathrm{CF}}=5,1 \mathrm{~Hz} \\ 158,4 & \underline{\mathrm{CN}} & \mathrm{d} & { }^{3} \mathrm{~J}_{\mathrm{CF}}=1,3 \mathrm{~Hz}\end{array}$

$\delta{ }^{19} \mathrm{~F}[\mathrm{ppm}] \quad 32,9 \quad$ SiFN $\quad \mathrm{S}$

$A B \underline{X}$ nicht aufgelöst

$\begin{array}{rrlll}\delta{ }^{29} \mathrm{Si}[\mathrm{ppm}] & -10,7 & \underline{\mathrm{SiFN}} & \mathrm{d} & { }^{1} \mathrm{~J}_{\mathrm{SiF}}=262 \mathrm{~Hz} \\ 1,6 & \mathrm{CS}\left(\mathrm{CH}_{3}\right)_{3} & \mathrm{~d} & { }^{3} \mathrm{~J}_{\mathrm{SiF}}=6,6 \mathrm{~Hz} \\ 5,5 & \mathrm{~N} \underline{\mathrm{Si}}\left(\mathrm{CH}_{3}\right)_{3} & \mathrm{~d} & { }^{3} \mathrm{~J}_{\mathrm{SiF}}=3,8 \mathrm{~Hz}\end{array}$

*: zugeordnet wurde nur das Hauptisomer 


\subsection{Röntgenstrukturdaten}

Im Nachfolgenden sind die gesammelten röntgenspektroskopischen Daten der Verbindungen 6, 13, 22, 27, 28, 34, 36, 38 und 39 aufgeführt. In den Tabellen finden sich charakteristische Daten und genauere Angaben zu der Messung. Daran schließt sich für jede Verbindung eine Abbildung des Ortep-Plots mit anisotropen Auslenkungsparametern mit 50 \%iger Wahrscheinlichkeit an. 


\begin{tabular}{|c|c|c|c|}
\hline Verbindung & 6 & 13 & 22 \\
\hline Temperatur [K] & $200(2)$ & $200(2)$ & $200(2)$ \\
\hline Wellenlänge [pm] & 71,073 & 71,073 & 71,073 \\
\hline Kristallsystem & Monoklin & Monoklin & Triklin \\
\hline Raumgruppe & $\mathrm{C} 2 / \mathrm{c}$ & $\mathrm{P} 2(1) / \mathrm{c}$ & $P-1$ \\
\hline Zelldimensionen (a) & $2592,0(5) \mathrm{pm}$ & $621,22(12) \mathrm{pm}$ & $1025,3(2) \mathrm{pm}$ \\
\hline (b) & $2003,4(4) p m$ & $1619,9(3) \mathrm{pm}$ & $1500,2(3) \mathrm{pm}$ \\
\hline (c) & $2087,3(4) \mathrm{pm}$ & $2434,7(5) \mathrm{pm}$ & $2006,4(4) \mathrm{pm}$ \\
\hline$(\alpha)$ & $90^{\circ}$ & $90^{\circ}$ & $74,16(3)^{\circ}$ \\
\hline$(\beta)$ & $126,06(3)^{\circ}$ & $97,33(3)^{\circ}$ & $83,86(3)^{\circ}$ \\
\hline$(\mathrm{Y})$ & $90^{\circ}$ & $90^{\circ}$ & $72,98(3)^{\circ}$ \\
\hline Zellvolumen $\left[\mathrm{nm}^{3}\right]$ & $8,76(1)$ & $2,301(8)$ & $2,8378(10)$ \\
\hline Z & 4 & 4 & 4 \\
\hline Dichte (ber.) $\left[\mathrm{Mg} / \mathrm{m}^{3}\right]$ & 1,057 & 1,109 & 1,079 \\
\hline Absorptionskoeffizient & $0,118 \mathrm{~mm}^{-1}$ & $0,169 \mathrm{~mm}^{-1}$ & $0,151 \mathrm{~mm}^{-1}$ \\
\hline$F(000)$ & 3072 & 888 & 1016 \\
\hline Kristallgröße $\left[\mathrm{mm}^{3}\right]$ & $1,00 \times 1,00 \times 0,80$ & $1,00 \times 0,90 \times 0,80$ & $1,00 \times 0,80 \times 0,40$ \\
\hline Gem. $\theta$-Bereich & $3,57^{\circ}-20,01^{\circ}$ & $3,54^{\circ}-25,04^{\circ}$ & $3,52^{\circ}-22,47^{\circ}$ \\
\hline Indexgrenzen (h) & $-24 \leq h \leq 24$ & $-7 \leq h \leq 7$ & $-10 \leq h \leq 11$ \\
\hline$(k)$ & $-19 \leq k \leq 19$ & $-19 \leq k \leq 19$ & $-15 \leq k \leq 16$ \\
\hline (l) & $-20 \leq 1 \leq 20$ & $-10 \leq \mathrm{I} \leq 29$ & $-19 \leq I \leq 21$ \\
\hline Anzahl Reflexe & 8142 & 3008 & 9082 \\
\hline Unabhängige Reflexe & $\begin{array}{c}4073 \\
{[R \text { (int) }=0,1332]}\end{array}$ & $\begin{array}{c}2361 \\
{[R \text { (int) }=0,1608]}\end{array}$ & $\begin{array}{c}7326 \\
{[R(\text { int })=0,1146]}\end{array}$ \\
\hline Vollst. Zu $\theta \approx 25^{\circ}$ & $99,3 \%$ & $54,6 \%$ & $99,4 \%$ \\
\hline Max. \& min. Transm. & $0,9115-0,8911$ & $0,8767-0,8493$ & $0,9422-0,8639$ \\
\hline $\begin{array}{c}\text { Daten / Restraints / } \\
\text { Parameter }\end{array}$ & 4073 / 12 / 400 & $2361 / 175$ / 248 & $7326 / 0$ / 570 \\
\hline Goodness-of-fit an $\mathrm{F}^{2}$ & 1,066 & 1,438 & 1,041 \\
\hline $\begin{array}{l}\text { Endgültige R-Werte } \\
\qquad[1>2 \sigma(I)]\end{array}$ & $\begin{array}{c}R 1=0,0961 \\
w R 2=0,2521\end{array}$ & $\begin{array}{c}\mathrm{R} 1=0,1139 \\
\mathrm{wR} 2=0,3173\end{array}$ & $\begin{array}{c}\mathrm{R} 1=0,0653 \\
w R 2=0,1826\end{array}$ \\
\hline $\begin{array}{l}\text { R-Werte (sämtlicher } \\
\text { Daten) }\end{array}$ & $\begin{array}{c}R 1=0,1223 \\
w R 2=0,2853\end{array}$ & $\begin{array}{c}R 1=0,1224 \\
w R 2=0,3379\end{array}$ & $\begin{array}{c}R 1=0,0817 \\
w R 2=0,2037\end{array}$ \\
\hline $\begin{array}{l}\text { Max. Differenz }\left[e . \AA^{3}\right] \\
\quad(\max .-\min .)\end{array}$ & $0,634--0,558$ & $1,310--0,497$ & $0,481--0,414$ \\
\hline
\end{tabular}




\begin{tabular}{|c|c|c|c|}
\hline Verbindung & 27 & 28 & 34 \\
\hline Temperatur [K] & $200(2)$ & $200(2)$ & $200(2)$ \\
\hline Wellenlänge [pm] & 71,073 & 71,073 & 71,073 \\
\hline Kristallsystem & Triklin & Triklin & Monoklin \\
\hline Raumgruppe & $P-1$ & $P-1$ & $\mathrm{P} 2(1) / \mathrm{n}$ \\
\hline Zelldimensionen (a) & $901,0(4) \mathrm{pm}$ & $892,2(4) \mathrm{pm}$ & $2029,2(5) \mathrm{pm}$ \\
\hline (b) & $1196,7(6) \mathrm{pm}$ & $1188,1(5) \mathrm{pm}$ & $1175,3(3) \mathrm{pm}$ \\
\hline (c) & $1294,0(6) \mathrm{pm}$ & $1268,9(6) \mathrm{pm}$ & $2134,0(6) \mathrm{pm}$ \\
\hline$(\alpha)$ & $98,37(3)^{\circ}$ & $96,49(4)^{\circ}$ & $90^{\circ}$ \\
\hline$(\beta)$ & $103,84(2)^{\circ}$ & $104,49,70(2)^{\circ}$ & $116,81(2)^{\circ}$ \\
\hline$(\mathrm{Y})$ & $110,87(2)^{\circ}$ & $111,957(19)^{\circ}$ & $90^{\circ}$ \\
\hline Zellvolumen $\left[\mathrm{nm}^{3}\right]$ & $1,2245(10)$ & $1,1753(9)$ & $4,54(1)$ \\
\hline Z & 2 & 2 & 4 \\
\hline Dichte (ber.) $\left[\mathrm{Mg} / \mathrm{m}^{3}\right]$ & 1,090 & 1,090 & 1,032 \\
\hline Absorptionskoeffizient & $0,208 \mathrm{~mm}^{-1}$ & $0,166 \mathrm{~mm}^{-1}$ & $0,184 \mathrm{~mm}^{-1}$ \\
\hline$F(000)$ & 440 & 424 & 1560 \\
\hline Kristallgröße $\left[\mathrm{mm}^{3}\right]$ & $1,00 \times 1,00 \times 0,80$ & $1,00 \times 1,00 \times 0,40$ & $1,00 \times 0,80 \times 0,70$ \\
\hline Gem. $\theta$-Bereich & $3,52^{\circ}-25,03^{\circ}$ & $3,53^{\circ}-25,02^{\circ}$ & $3,50^{\circ}-22,49^{\circ}$ \\
\hline Indexgrenzen (h) & $-10 \leq h \leq 10$ & $-10 \leq h \leq 10$ & $-21 \leq h \leq 21$ \\
\hline$(k)$ & $-14 \leq k \leq 14$ & $-14 \leq k \leq 14$ & $-12 \leq k \leq 12$ \\
\hline (l) & $-15 \leq \mathrm{I} \leq 15$ & $-15 \leq \mathrm{I} \leq 15$ & $-22 \leq 1 \leq 22$ \\
\hline Anzahl Reflexe & 8640 & 6699 & 9814 \\
\hline Unabhängige Reflexe & $\begin{array}{c}4320 \\
{[R(\text { int })=0,0768]}\end{array}$ & $\begin{array}{c}4138 \\
{[R(\text { int })=0,0847]}\end{array}$ & $\begin{array}{c}5918 \\
{[R(\text { int })=0,0274]}\end{array}$ \\
\hline Vollst. $\mathrm{Zu} \theta \approx 25^{\circ}$ & $99,5 \%$ & $99.7 \%$ & $99,6 \%$ \\
\hline Max. \& min. Transm. & $0,8514-0,8191$ & $0,9367-0,8518$ & $0,8820-0,8374$ \\
\hline $\begin{array}{c}\text { Daten / Restraints / } \\
\text { Parameter }\end{array}$ & 4320 / 0 / 238 & 4138 / 0 / 238 & 5918 / 0 / 430 \\
\hline Goodness-of-fit an $\mathrm{F}^{2}$ & 1,050 & 1,046 & 1,033 \\
\hline $\begin{array}{l}\text { Endgültige R-Werte } \\
\qquad[1>2 \sigma(I)]\end{array}$ & $\begin{array}{c}R 1=0,0408 \\
w R 2=0,1083\end{array}$ & $\begin{aligned} \mathrm{R} 1 & =0,0451 \\
\mathrm{wR} 2 & =0,01211\end{aligned}$ & $\begin{array}{c}R 1=0,0380 \\
w R 2=0,0918\end{array}$ \\
\hline $\begin{array}{l}\text { R-Werte (sämtlicher } \\
\text { Daten) }\end{array}$ & $\begin{array}{c}\mathrm{R} 1=0,0428 \\
w R 2=0,1106\end{array}$ & $\begin{array}{c}\mathrm{R} 1=0,0470 \\
\mathrm{wR} 2=0,1235\end{array}$ & $\begin{array}{c}\mathrm{R} 1=0,0488 \\
\mathrm{wR} 2=0,0998\end{array}$ \\
\hline $\begin{array}{l}\text { Max. Differenz }\left[e . \AA^{3}\right] \\
\quad(\max .-\min .)\end{array}$ & $0,455--0,287$ & $0,317--0,317$ & $0,273--0,259$ \\
\hline
\end{tabular}




\begin{tabular}{|c|c|c|c|}
\hline Verbindung & 36 & 38 & 39 \\
\hline Temperatur [K] & $200(2)$ & $133(2)$ & 203(2) \\
\hline Wellenlänge [pm] & 71,073 & 71,073 & 71,073 \\
\hline Kristallsystem & Triklin & Monoklin & Monoklin \\
\hline Raumgruppe & $P-1$ & $\mathrm{P} 2(1) / \mathrm{c}$ & $\mathrm{P} 2(1) / \mathrm{c}$ \\
\hline Zelldimensionen (a) & $935,0(3) \mathrm{pm}$ & $1990,8(2) \mathrm{pm}$ & $1218,5(4) \mathrm{pm}$ \\
\hline (b) & $1132,7(5) \mathrm{pm}$ & $2716,8(5) \mathrm{pm}$ & $1202,8(3) \mathrm{pm}$ \\
\hline (c) & $1434,9(17) \mathrm{pm}$ & $1187,7(2) \mathrm{pm}$ & $1949,7(12) \mathrm{pm}$ \\
\hline$(\alpha)$ & $80,65(3)^{\circ}$ & $90^{\circ}$ & $90^{\circ}$ \\
\hline$(\beta)$ & $74,36(4)^{\circ}$ & $90,24(3)^{\circ}$ & $95,86(7)^{\circ}$ \\
\hline$(\mathrm{Y})$ & $80,278(15)^{\circ}$ & $90(3)^{\circ}$ & $90^{\circ}$ \\
\hline Zellvolumen $\left[\mathrm{nm}^{3}\right]$ & $1,4310(19)$ & $6,424(2)$ & $2,84(1)$ \\
\hline Z & 2 & 8 & 4 \\
\hline Dichte (ber.) $\left[\mathrm{Mg} / \mathrm{m}^{3}\right]$ & 1,019 & 1,036 & 1,070 \\
\hline Absorptionskoeffizient & $0,177 \mathrm{~mm}^{-1}$ & $0,165 \mathrm{~mm}^{-1}$ & $0,186 \mathrm{~mm}^{-1}$ \\
\hline$F(000)$ & 488 & 2208 & 1008 \\
\hline Kristallgröße $\left[\mathrm{mm}^{3}\right]$ & $1,00 \times 0,80 \times 0,16$ & - & $1,00 \times 1,00 \times 0,80$ \\
\hline Gem. $\theta$-Bereich & $3,59^{\circ}-25,01^{\circ}$ & $1,27^{\circ}-25,73^{\circ}$ & $3,55^{\circ}-22,44^{\circ}$ \\
\hline Indexgrenzen (h) & $-11 \leq h \leq 11$ & $-24 \leq h \leq 24$ & $-13 \leq h \leq 13$ \\
\hline$(k)$ & $-13 \leq k \leq 13$ & $0 \leq \mathrm{k} \leq 32$ & $-4 \leq k \leq 12$ \\
\hline (I) & $-17 \leq \mathrm{I} \leq 17$ & $0 \leq 1 \leq 14$ & $-10 \leq \mathrm{I} \leq 20$ \\
\hline Anzahl Reflexe & 9985 & 106238 & 3700 \\
\hline Unabhängige Reflexe & $\begin{array}{c}4993 \\
{[R \text { (int) }=0,1215]}\end{array}$ & $\begin{array}{c}12065 \\
{[R \text { (int) }=0,1173]}\end{array}$ & $\begin{array}{c}3669 \\
{[R(\text { int })=0,1408]}\end{array}$ \\
\hline Vollst. $\mathrm{Zu} \theta \approx 25^{\circ}$ & $99,3 \%$ & $98,2 \%$ & $99,6 \%$ \\
\hline Max. \& min. Transm. & $0,9723-0,8430$ & - & $0,8653-0,8357$ \\
\hline $\begin{array}{c}\text { Daten / Restraints / } \\
\text { Parameter }\end{array}$ & 4993 / 0 / 269 & 12065 / 0 / 627 & 3669 / 0 / 277 \\
\hline Goodness-of-fit an $\mathrm{F}^{2}$ & 1,028 & 1,023 & 1,042 \\
\hline $\begin{array}{l}\text { Endgültige R-Werte } \\
\qquad[1>2 \sigma(I)]\end{array}$ & $\begin{array}{c}R 1=0,0416 \\
w R 2=0,1091\end{array}$ & $\begin{array}{c}\mathrm{R} 1=0,0396 \\
\mathrm{wR} 2=0,0975\end{array}$ & $\begin{array}{c}\mathrm{R} 1=0,0413 \\
\mathrm{wR} 2=0,1106\end{array}$ \\
\hline $\begin{array}{l}\text { R-Werte (sämtlicher } \\
\text { Daten) }\end{array}$ & $\begin{array}{c}\mathrm{R} 1=0,0446 \\
w R 2=0,1126\end{array}$ & $\begin{array}{c}R 1=0,0461 \\
w R 2=0,1006\end{array}$ & $\begin{array}{c}\mathrm{R} 1=0,0449 \\
\mathrm{wR} 2=0,1144\end{array}$ \\
\hline $\begin{array}{l}\left.\text { Max. Differenz [e. } \AA^{3}\right] \\
\quad(\max .-\min .)\end{array}$ & $0,402--0,328$ & $0,448--0,386$ & $0,299--0,276$ \\
\hline
\end{tabular}




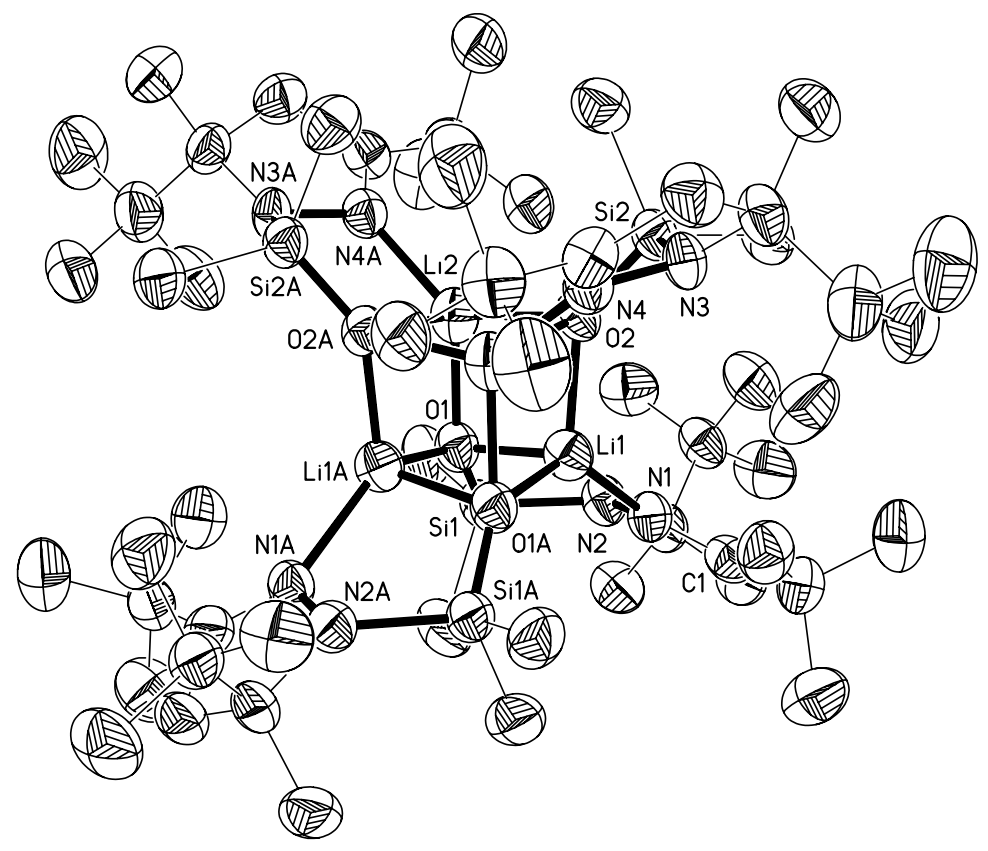

Ortep-Plot 1: Kristallstruktur von Verbindung 6

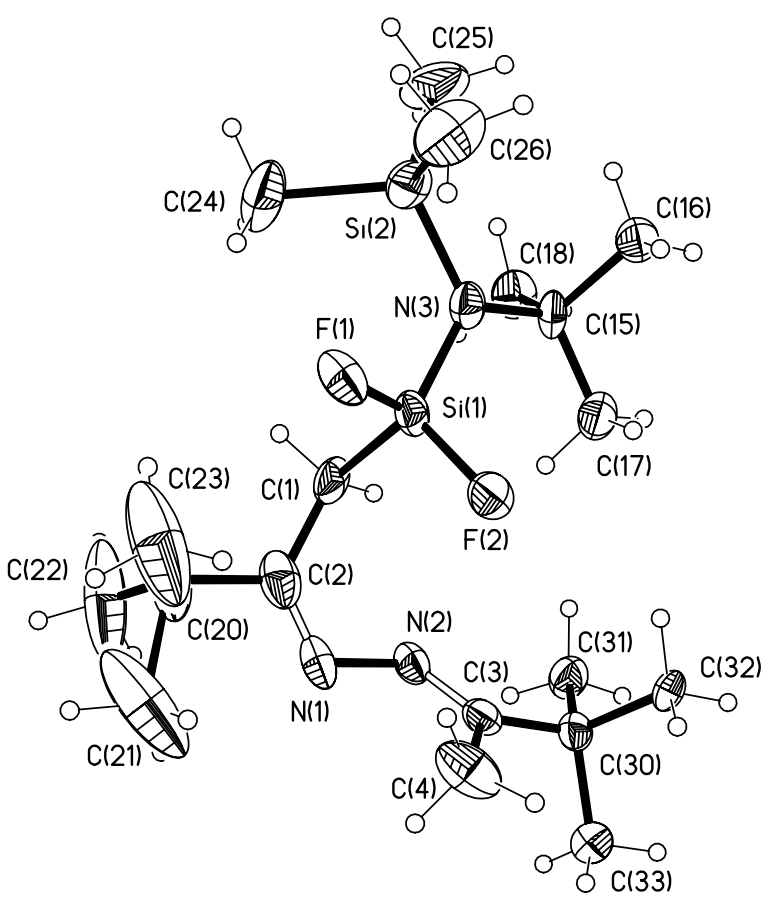

Ortep-Plot 2: Kristallstruktur von Verbindung 13 


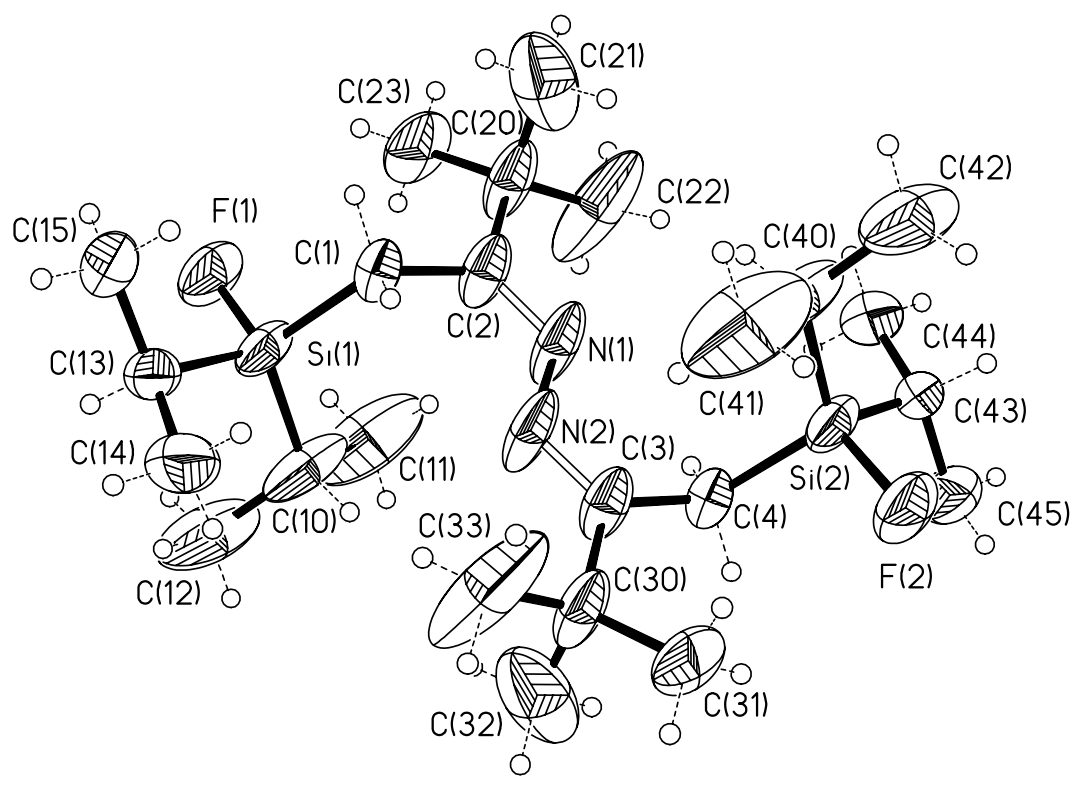

Ortep-Plot 3: Kristallstruktur von Verbindung 22

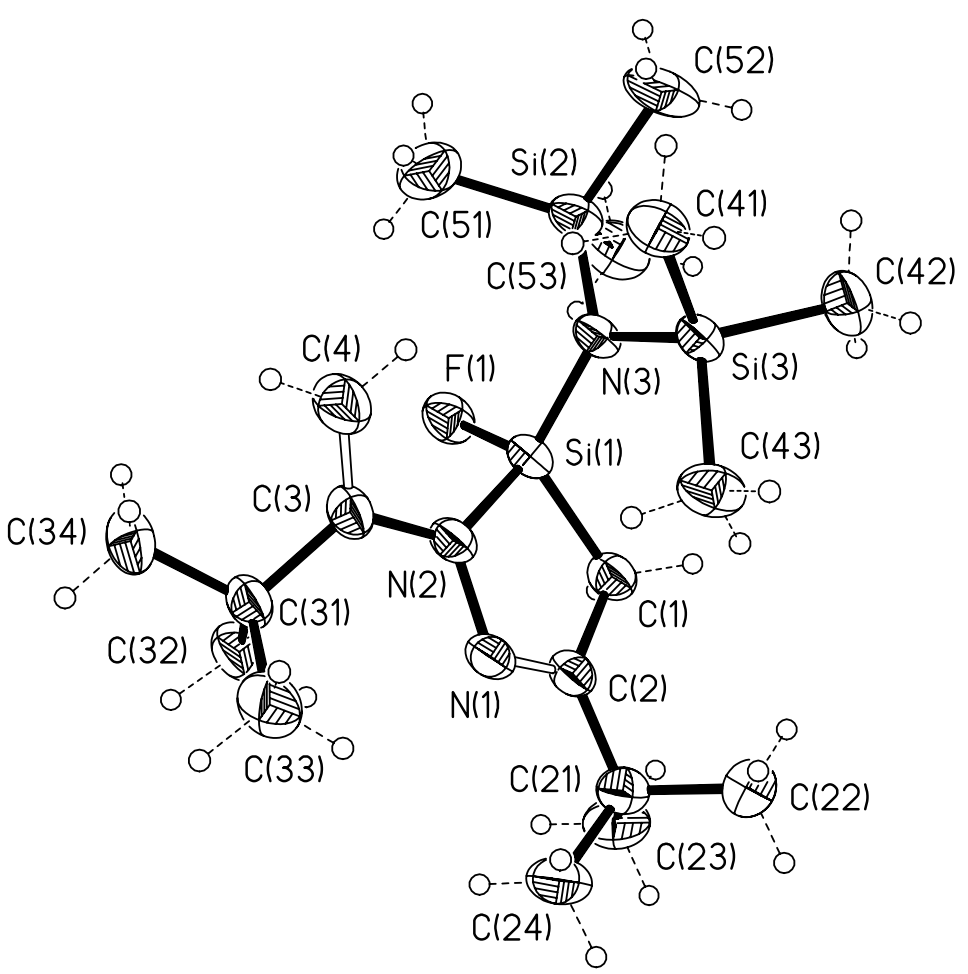

Ortep-Plot 4: Kristallstruktur von Verbindung 27 


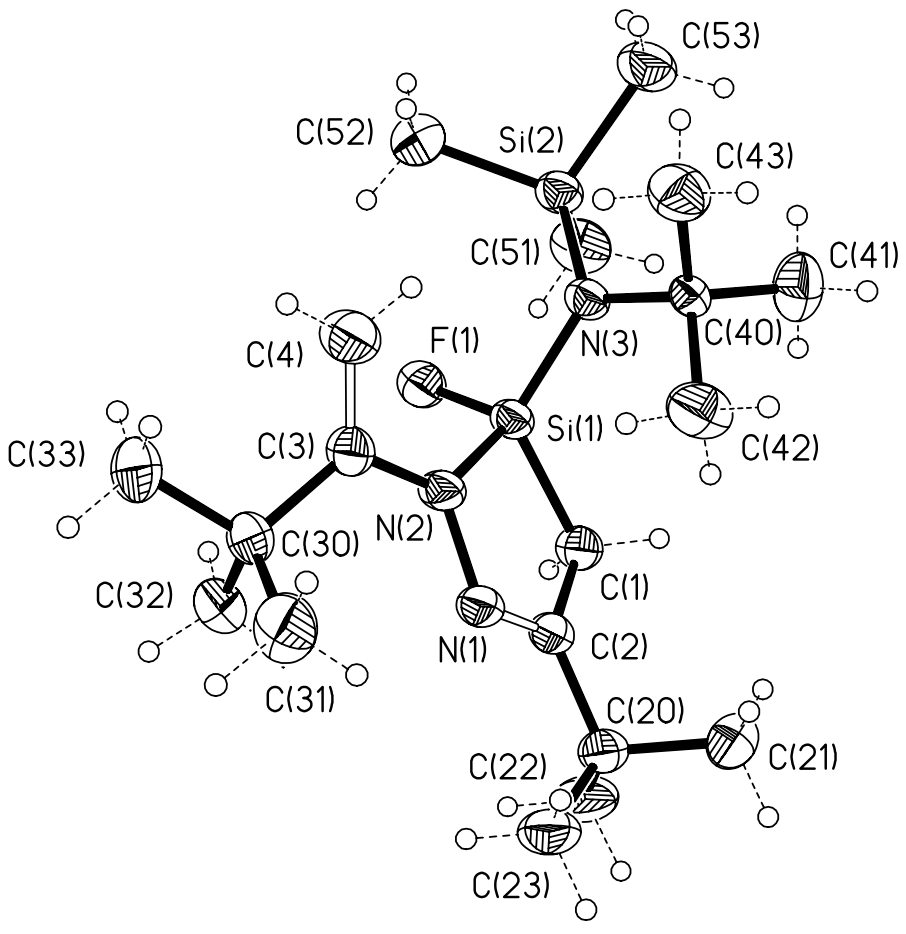

Ortep-Plot 5: Kristallstruktur von Verbindung 28

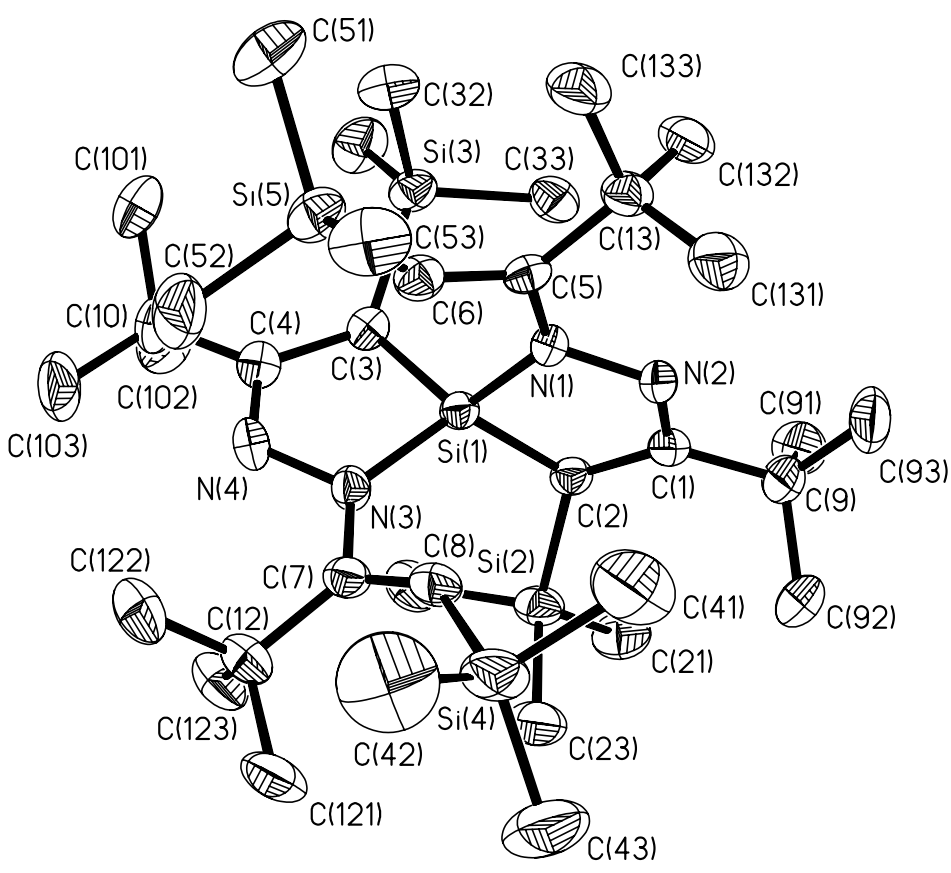

Ortep-Plot 6: Kristallstruktur von Verbindung 34 


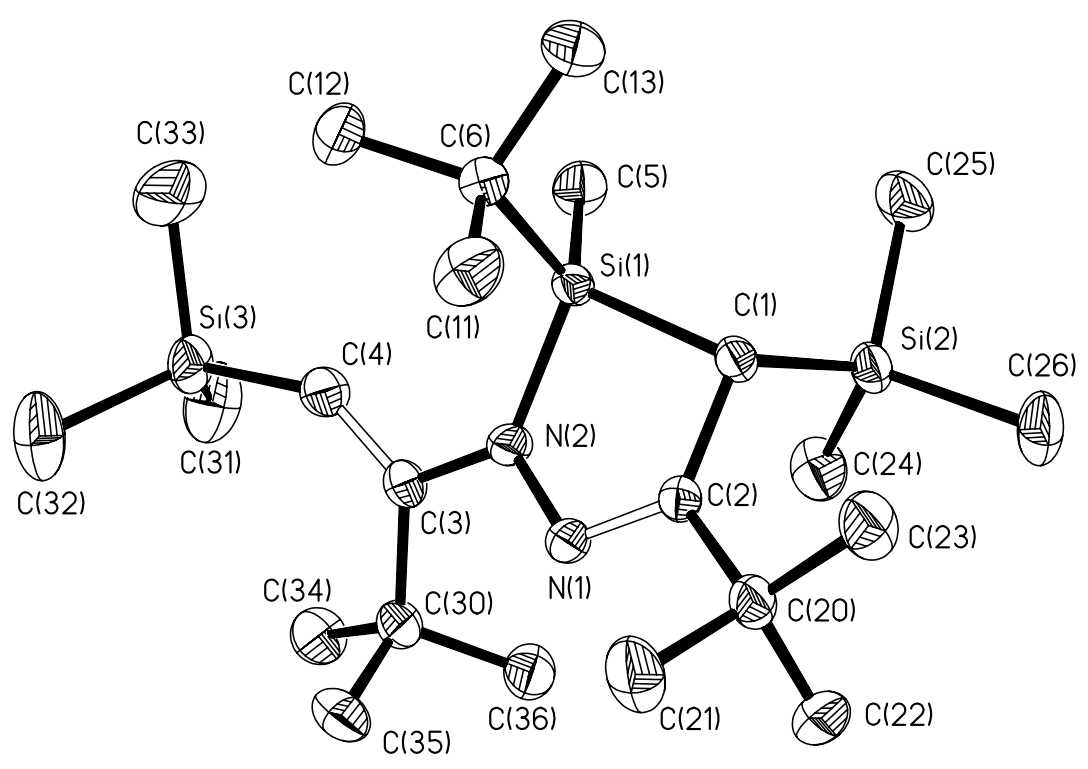

Ortep-Plot 7: Kristallstruktur von Verbindung 36

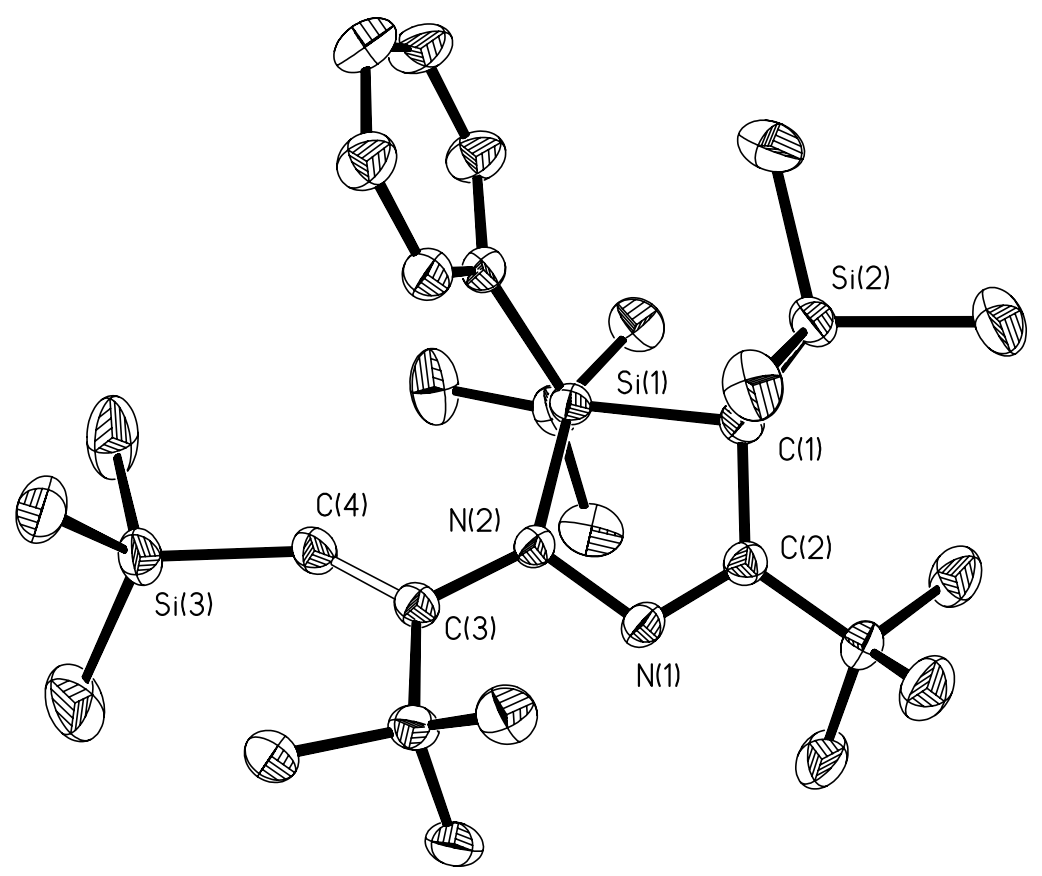

Ortep-Plot 8: Kristallstruktur von Verbindung 38 


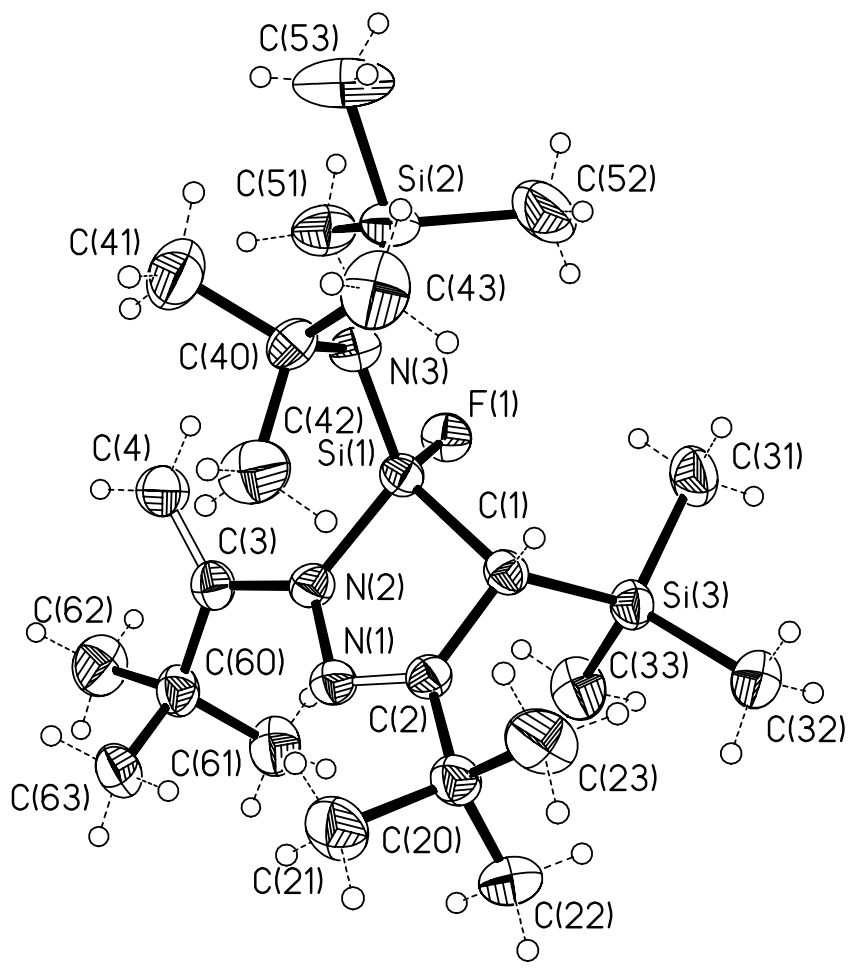

Ortep-Plot 9: Kristallstruktur von Verbindung 39 


\subsection{Verzeichnis der neuen Verbindungen}<smiles>C/C(CC(C)(C)C)=N/N=C(/C)C(C)(C)C</smiles>

1<smiles>C/C(=N\N=C(/C[Si](C)(C)Cl)C(C)(C)C)C(C)(C)C</smiles>

2<smiles>C/C(=N/N=C(/C[Si](C)(Cl)c1ccccc1)C(C)(C)C)C(C)(C)C</smiles>

3

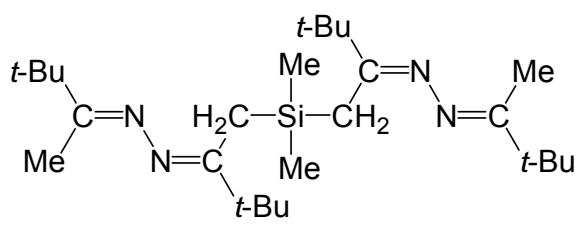

4

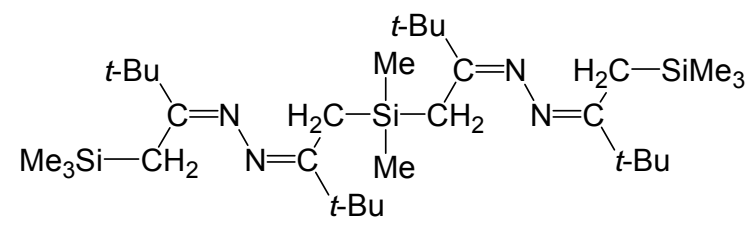

5

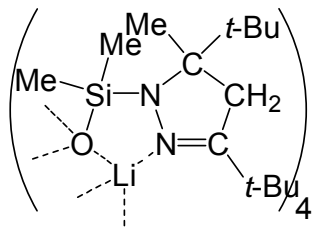

6<smiles>C/C(=N\N=C(/C[Si](Cl)(Cl)c1ccccc1)C(C)(C)C)C(C)(C)C</smiles>

7<smiles>CC(=NN=C(C[Si](Cl)(Cl)Cl)C(C)(C)C)C(C)(C)C</smiles>

8<smiles>CC(=NN=C(C[Si](C)(C)c1ccccc1)C(C)(C)C)C(C)(C)C</smiles>

9

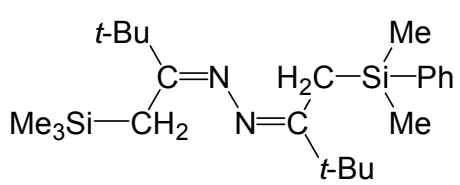

10

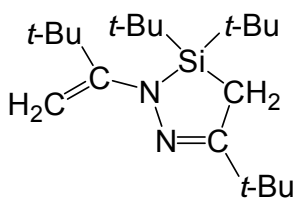

11<smiles>CC(C)/C(=N\N1C([13CH2]C(C)(C)C)=C[Si]1(C(C)(C)C)C(C)(C)C)C(C)(C)C</smiles><smiles>C/C(=N/N=C(/C[Si](F)(N(C)C(C)(C)C)C(C)(C)C)C(C)(C)C)C(C)(C)C</smiles><smiles>C/C(=N/N=C(/C[Si](F)(CC(C)(C)C)CC(C)(C)C)N/N=C(\C)C(C)(C)C)C(C)(C)C</smiles>

14 
<smiles>C=C(N1N=C(C(C)(C)C)C[Si]1(C)C)C(C)(C)C</smiles>

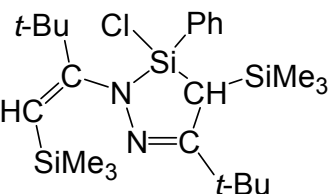

18<smiles>C=C(N1N=C(C(C)(C)C)C[Si]1(C(C)C)C(C)C)C(C)(C)C</smiles>

21<smiles>CC(C)(C)C1=CC(C(C)(C)C)C[Si](C(C)(C)C)(C(C)(C)C)NN1</smiles>

24<smiles>C=C(N1N=C(C(C)(C)C)C[Si]1(F)N(C)[SiH3])C(C)(C)C</smiles><smiles>C=C(N1N=C(C(C)(C)C)C[Si]1(C)c1ccccc1)C(C)(C)C</smiles>

17<smiles>C=C(N1N=C(C(C)(C)C)C[Si]1(Cl)Cl)C(C)(C)C</smiles>

19<smiles>C[SiH2]/C=C(/N1N=C(C(C)(C)C)[C@H]([AsH3])[Si]1(Cl)Cl)C(C)(C)C</smiles>

20

23<smiles>CS/C=C(\N1N=C(C(C)(C)C)C[Si]1(C(C)(C)C)C(C)(C)C)C(C)(C)C</smiles>

25<smiles>C=C(N1N=C(C(C)(C)C)C[Si]1(F)C(C)(C)C)C(C)(C)C</smiles>

26<smiles>CCC(C)C1=NN(/C(=C\C(C)C)C(C)(C)C)[Si](F)(C(C)(C)C)C1[13CH3]</smiles>

29 
<smiles>C=C(SC)C(N1N=C(C(C)(C)C)[Si](F)(c2ccccc2)C1[SiH3])C(C)(C)C</smiles>

30<smiles>C=C(N1N=C(C(C)(C)C)C[Si]12CC(C(C)(C)C)=NN2C(=C)C(C)(C)C)C(C)(C)C</smiles>

33<smiles>CCC(C)C1=NN(/C(=C\SC)C(C)(C)C)[Si](C)(C(C)(C)C)C1C(C)(C)C</smiles>

36<smiles>C=C(N1N=C(C(C)(C)C)[C@H](C(C)(C)C)[Si]1(F)N(C)C(C)(C)C)C(C)(C)C</smiles>

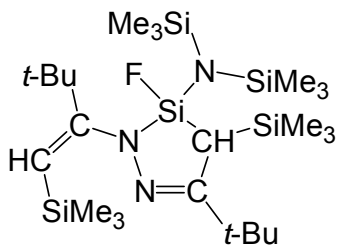

31

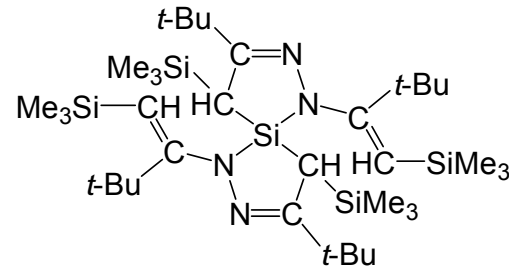

34

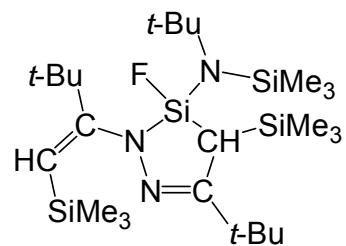

32<smiles>C/C(=N\N=C(/C[R15](C)(C)C)C[Si](CC(C)(C)C)(OC(C)(C)C)C(C)(C)C)C(C)(C)C</smiles>

35 


\section{Literaturverzeichnis}

[1] Houben-Weyl: Methoden der organischen Chemie. Organische Stickstoffverbindungen mit einer C, N-Doppelbindung. Teil 2. Bd. E14b. S. 640. Stuttgart · New York: 1990. Georg Thieme Verlag.

[2] Houben-Weyl: Methoden der organischen Chemie. Ketone II. Band 7/2b. S. 1954. Stuttgart · New York: 1976. Georg Thieme Verlag.

[3] Houben-Weyl: Methoden der organischen Chemie. Stickstoffverbindungen I. Teil 2. Bd. 10/2. S. 89. Stuttgart · New York: 1976. Georg Thieme Verlag.

[4] E. Lederer; Bull. Soc. Chim. Fr., 1946, 172.

[5] E. Arnal, J. Elguero, R. Jacquier, C. Marzin, J. Wylde; Bull. Soc. Chim. Fr., $1967,877$.

[6] K. Appenroth, M. Reichenbaecher, R. Paetzold; Tetrahedron, 1981, 37, 569.

[7] D. S. Malament, J. M. McBride; J. Am. Chem. Soc., 1970, 92, 4586.

[8] F. E. Henoch, K. G. Hampton, C. R. Hauser; J. Am. Chem. Soc., 1969, 91, 676.

[9] T. Groh, G. Elter, M. Noltemeyer, H.-G. Schmidt, A. Meller; Organometallics, 2000, 19, 2477.

[10] T. Groh, G. Elter, M. Noltemeyer, H.-G. Schmidt, A. Meller; Main Group Metal Chemistry, 2000, 23, No. 11, 709.

[11] F. Armbruster; Staatsexamensarbeit, Göttingen 2001.

[12] B. J. Aylett; J. Inorg. Nucl. Chem., 1956, 2, 325.

[13] U. Wannagat, W. Liehr; Angew. Chem., 1957, 69, 783.

U. Wannagat, W. Liehr; Z. anorg. allg. Chem., 1958, 297, 129.

[14] U. Wannagat, F. Höfler, H. Bürger; Monatsh. Chem., 1966, 96, 2038.

U. Wannagat, H. Niederprüm; Angew. Chem., 1959, 71, 574.

[15] U. Wannagat; Adv. of Inorg. Radiochem., 1964, 6, 225.

[16] N. W. Mitzel, P. Bissinger, H. Schmidbaur; Chem. Ber., 1993, 126, 345.

[17] C. Drost, U. Klingebiel ; Chem. Ber., 1993, 126, 1413.

[18] S. Dielkus, C. Drost, R. Herbst-Irmer, U. Klingebiel; Angew. Chem., 1993, 105(11), 1689. Angew. Chem. Int. Ed. Engl., 1993, 32, 1625.

[19] S. Dielkus, C. Drost, R. Herbst-Irmer, U. Klingebiel; Organometallics, 1994, 13, 3985. 
[20] K. Bode, U. Klingebiel, H. Witte-Abel, M. W. Gluth, M. Noltemeyer, R. HerbstIrmer, M. Schäfer; W. Shomaly: Phosphorus, Sulfur and Silicon and the Related Elements, 1996, 108 (1-4), 121.

[21] C. Drost; Dissertation, Göttingen, 1993.

[22] U. Klingebiel, S. Schmatz, E. Gellermann, C. Drost, M. Noltemeyer; Monatshefte für Chemie, 2001, 132, 1105.

[23] J. R. Dilworth, I. A. Latham, G. J. Leigh, G. Huttner, I. Jibril; J. Chem. Soc., Chem. Commun., 1983, 22, 1368.

[24] R. E. Bailey, R. West; J. Am. Chem. Soc., 1964, 86, 5369.

[25] R. West, M. Ishikawa, R. E. Bailey; J. Am. Chem. Soc., 1966, 88, 4648.

[26] R. West, M. Ishikawa, R. E. Bailey; J. Am. Chem. Soc., 1967, 89, 4068.

[27] R. West, M. Ishikawa, R. E. Bailey; J. Am. Chem. Soc., 1967, 89, 4981.

[28] R. West, M. Ishikawa, R. E. Bailey; J. Am. Chem. Soc., 1967, 89, 4072.

[29] R. West, B. Bichlmeir; J. Am. Chem. Soc., 1972, 94, 1649.

[30] M. J. S. Dewar, W. B. Jennings; J. Am. Chem. Soc., 1973, 95, 1562.

[31] J. R. Fletcher, J. O. Sutherland; J. Chem. Soc., Chem. Commun., 1970, 687.

[32] J. O. Scherer, U. Bultjer; Angew. Chem., 1971, 83, 364. Angew. Chem. Int. Ed. Engl., 1971, 10(5), 343.

[33] H. Kessler; Angew. Chem., 1970, 52, 237. Angew. Chem. Int. Ed. Engl., 1970, 9(3), 219.

[34] M. Veith, H. Bäringhausen; Acta Cryst., 1974, B30, 1806.

[35] N. Wiberg, W. Ch. Joo, W. Uhlenbrock; Angew. Chem., 1968, 80, 661. Angew. Chem. Int. Ed. Engl., 1968, 7(8), 640.

[36] N. Wiberg, M. Veith; Chem. Ber., 1971, 104, 3176.

[37] K. Bode, U. Klingebiel, M. Noltemeyer, H. Witte-Abel; Z. anorg. allg. Chem., 1995, 621, 500 .

[38] C. Drost, U. Klingebiel, M. Noltemeyer; J. Organomet. Chem., 1991, 414, 307.

[39] K. Seppelt, W. Sundermeyer; Chem. Ber., 1969, 102, 1247.

[40] K. Bode, U. Klingebiel; Adv. Organomet. Chem., 1996, 40, 1.

[41] K. Bode, C. Drost, C. Jäger, U. Klingebiel, M. Noltemeyer, J. Zdirad Zak; J. Organomet. Chem., 1994, 482, 285.

[42] U. Klingebiel, P. Werner; Liebigs Ann. Chem., 1979, 4, 457.

[43] U. Klingebiel, P. Werner; J. Organomet. Chem., 1979, 178, 409.

[44] W. Clegg, M. Noltemeyer, G. M. Sheldrick; Acta Cryst., 1979, B 35, 2243. 
[45] U. Klingebiel, S. Pohlmann, P. Werner; Liebigs Ann. Chem., 1980, 11, 1898.

[46] U. Schneider, Staatsexamensarbeit, Göttingen, 1986.

[47] K. Knipping, C. Drost, U. Klingebiel, M. Noltemeyer; Z. anorg. allg. Chem., 1996, 622, 1215.

[48] U. Klingebiel, S. Pohlmann, P. Werner; Z. anorg. allg. Chem., 1981, 482, 65.

[49] M. Hesse, U. Klingebiel; Z. anorg. allg. Chem., 1983, 501, 57.

[50] W. Clegg, U. Klingebiel, G. M. Sheldrick, P. Werner; Angew. Chem., 1981, 93, 391. Angew. Chem. Int. Ed. Engl., 1981, 20, 384.

[51] W. Clegg, O. Graalmann, M. Haase, U. Klingebiel, G. M. Sheldrick, P. Werner, G. Henkel, B. Krebs; Chem. Ber., 1983, 116, 282.

[52] W. Clegg, U. Klingebiel, S. Pohlmann, G. M. Sheldrick, P. Werner; Angew. Chem., 1981, 93, 390. Angew. Chem. Int. Ed. Engl., 1981, 20, 383.

[53] W. Clegg, M. Haase, M. Hesse, U. Klingebiel, G. M. Sheldrick; Angew. Chem., 1982, 94, 461. Angew. Chem. Int. Ed. Engl., 1982, 21, 445. Angew. Chem. Suppl., 1982, 1009.

[54] A. Schmidpeter, K. Karaghiosoff; in H.W. Roesky (Ed.): Rings, Clusters and Polymers of Main Group and Transition Elements. Amsterdam · New York: 1989. Elsevier Verlag.

[55] H. Niederprüm, U. Wannagat; Z. anorg. allg. Chem., 1961, 311, 270.

[56] Z. J. Sergeeva, Tszyan Sin-Chzhan, D. D. Tsitovich; J. Allg. Chem., 1960, 30, 694.

[57] U. Klingebiel, H. Hluchy, A. Meller; Chem. Ber., 1978, 111, 906.

[58] K. Bode, U. Klingebiel, M. Noltemeyer, H. Witte-Abel; Z. anorg. allg. Chem., $1995,621,500$.

[59] A. G. Brook, S. C. Nyburg, F. Abdesaken, B. Gutekunst, G. Gutekunst, R. Krishna, M. R. Kallury, Y. C. Poon, Y.-M. Chang, W. Wong-Ng; J. Am. Chem. Soc., 1982, 104, 5667.

[60] N. Wiberg, G. Wagner; Angew. Chem., 1983, 95, Nr. 12, 1027. Angew. Chem. Int. Ed. Engl., 1983, 22(12), 1005.

[61] N. Wiberg, G. Wagner, G. Reber, J. Riede, G. Müller; Organometallics, 1987, 6,35 .

[62] N. Wiberg, K.-S. Joo, K. Polborn; ; Chem. Ber., 1993, 126, 67.

[63] M. Hesse, Dissertation, Göttingen, 1983.

[64] U. Schneider, Staatsexamensarbeit, Göttingen, 1986. 
[65] F. Armbruster, Dissertation, Göttingen, 2004.

[66] K. P. C. Vollhardt, N. E. Schore: Organische Chemie, 2. Auflage, S.271; Weinheim · New York · Basel · Cambridge · Tokyo: 1995. VCH Verlag.

[67] K. Gregory, P. R. von Schleyer, R. Snaith; Advances in Inorganic Chemistry, 1991, 37, 47.

[68] O. Graalmann, M. Hesse, U. Klingebiel, W. Clegg, M. Haase, G. M. Sheldrick; Z. anorg. allg. Chem., 1984, 514, 49.

[69] R. West, M. Denk; Pure and Applied Chemistry, 1996, 68, 785.

[70] T. Müller: A Model System for the Generation of Silyl Cationic Spezies of Different Reactivity and Stability. in: N. Auner, J. Weis: Organosilicon Chemistry V. Weinheim: 2003. Wiley-VCH, S. 34.

[71] P. D. Lickiss, P. C. Masangane, W. Sohal, G. L. Veneziani: Synthesis and Chemistry of Some Bridge Silicocations. in: N. Auner, J. Weis: Organosilicon Chemistry V. Weinheim: 2003. Wiley-VCH, S. 45.

[72] A. Bockholt, T. Braun, P. Jutzi, B. Neumann, A. Stammler, H.-G. Stammler: New Donor-Stabilized Organosilicon Cations: Synthesis, Strukture and Reactivity. in: N. Auner, J. Weis: Organosilicon Chemistry V. Weinheim: 2003. Wiley-VCH, S. 50.

[73] H. Berlekamp, P. Jutzi, B. Neumann, A. Mix, W. Schoeller, H.-G. Stammler; Angew. Chem., 1999, 111, 2071. Angew. Chem. Int. Ed. Engl., 1999, 38(13/14), 2048. 


\section{Publikationen}

N. Helmold, B. Jaschke, U. Klingebiel: Cyclosilazanes and their Application as Precursors for Silican-based Ceramics; Phosphorus, Sulfur, and Silicon, 2001, 169, 245.

N. Helmold, V. Liebau, S. Schmatz, U. Klingebiel: Isomeric Cyclosilazanes and their Application as Precursors for Silican-based Ceramics; Organosilicon Chemistry, ed. N. Auner and J. Weis, Weinheim, VCH, 2003, 5, 261.

B. Jaschke, N. Helmold, I. Müller, M. Noltemeyer, R. Herbst-Irmer, U. Klingebiel: Kopplung von Cyclosilazanen mit Aminofluorboranen und Borazinen; Z. anorgan. Allg. Chem., 2002, 628, 2071.

\section{Vorträge}

U. Klingebiel, N. Helmold, B. Jaschke: Cyclosilazanes and their Application as Precursors for Silican-based Ceramics; $9^{\text {th }}$ International Symposium on Inorganic Ring Systems, Saarbrücken, Germany, July 2000.

U. Klingebiel, N. Helmold, V. Liebau: Isomeric Cyclosilazanes and their Application as Precursors for Silican-based Ceramics; $1^{\text {st }}$ European Silicon Days, München, September 2001. 


\section{Lebenslauf}

Name:

Nina Armbruster, geb. Helmold

Geboren:

22.02.1973 in Göttingen

Schulbildung: $\quad 1979-1983$ Grundschule in Fuhrbach

1983 - 1985 Orientierungsstufe St.-Ursula-Schule Duderstadt

1985 - 1992 Eichsfeld-Gymnasium Duderstadt

19.05.1992 Erwerb der Allgemeinen Hochschulreife

Berufsausbildung: 1992 - 1995 Ausbildung zur Biologielaborantin am Inst. f. Tierphysiologie und Tierernährung der Georg-August-Universität Göttingen

28.06.1995 Prüfung durch die IHK Hannover-Hildesheim

Studium:

WS 95/96 Immatrikulation an der Georg-August-Universität Göttingen im Fach

Chemie und Biologie auf Lehramt (Sek. II)

1999 - 2001 Studentische Hilfskraft im Inst. f. Anorg. Chemie der Georg-August-Universität Göttingen

29.05.2001 Erstes Staatsexamen

Promotion: $\quad 2001-2004 \quad$ Experimentielle Promotionsarbeit im Institut für Anorganische Chemie Göttingen unter der Leitung von Prof. U. Klingebiel

2001 - 2004 Wissenschaftliche Hilfskraft bzw. Mitarbeiterin im Institut für Anorganische Chemie Göttingen 